\title{
Underestimating lifespans? Why longevity risk exists in retirement planning and superannuation policy
}

\author{
by
}

\section{Alison O'Connell}

\author{
A thesis \\ submitted to the Victoria University of Wellington \\ in fulfilment of the requirements for the degree of \\ Doctor of Philosophy
}

Victoria University of Wellington

(2012) 



\title{
Underestimating lifespans? Why longevity risk exists in retirement planning and superannuation policy
}

\begin{abstract}
The pace of increasing life expectancy in recent decades came as a surprise to demographers, as mortality rates unexpectedly improved at the oldest ages in developed countries. The most common policy response, although one not yet planned for New Zealand, is to increase eligibility age for the public pension. Given the complexity and uncertainty of processes driving mortality improvement, future lifespans cannot be known. However, it is questionable whether policy makers and individuals understand the extent of past and likely future lifespan increase. Available evidence suggests individuals tend to underestimate how long they may live. Population mortality forecasts are generally conservative and poorly explain longevity uncertainties. Longevity risk - the possibility that future lifespans will be longer than anticipated threatens individuals' pre-retirement financial planning and public pension policy.
\end{abstract}

This thesis examines the extent of longevity risk, its causes, significance and remedies, in these two domains, for New Zealand. The theoretical existence of longevity risk has been acknowledged, but has not been subject to critical analysis in New Zealand or elsewhere. Here, a unique generalisable methodology exploiting insights available from international mortality comparisons is designed, combining actuarial and demographic theory. After assessing the flaws in the time-dependent or period approach to measurement 
of life expectancy that are known in theory but underexplored in practice, the method emphasises the lifecourse or cohort approach. The three factors that determine longevity risk - plausible population lifespan prospects, the lifespan assumptions used by policy makers and individuals' subjective lifespan expectations - are identified and the relationships between them analysed for New Zealand. An interpretation of the consistency of New Zealand's past mortality trends and future projections with those of other British settler countries, supplemented by a review of the consequences of mortality variance within New Zealand, shows that plausible lifespans in New Zealand are likely to be higher than those in the official projections on which policy makers rely. The first survey to ask how long New Zealanders think they will live shows that collectively, New Zealanders are more likely to underestimate future lifespan than not, based on a variety of beliefs about mortality that are not consistent with the evidence on increasing lifespans.

Longevity risk from underestimation of future lifespans is revealed in New Zealand policy making and in individual New Zealanders' retirement plans. The most likely cause is the repeated misuse of life expectancy indicators in an environment lacking public discourse about increasing longevity. A remedy would be switching from using flawed period life expectancy indicators to using cohort life expectancy or modal age at death. Using plausible estimates for future lifespans based on more optimistic estimates than the official projections most often referenced would be important but mitigate longevity risk to a lesser extent. A more extensive public debate than has been held so far about eligibility age for New Zealand's public pension would itself, if using appropriate indicators for future lifespans, provide an opportunity to address longevity risk. 
Keywords: Life expectancy, mortality, longevity, pension policy, subjective longevity expectations.

\section{Acknowledgements}

I have been fortunate to work with a number of supportive colleagues in different settings over many years as my interest in longevity policy grew. I acknowledge (in alphabetical order): John Coomber FIA, Chris Curry, Baroness Greengross of Notting Hill, Tom Ross FFA and Steve Webb MP. I would like to thank Kim Dunstan and Adrian Gallop for their kindness as they willingly responded to requests for information about mortality in New Zealand and the UK respectively. I am grateful to David Feslier and Diana Crossan from the Commission for Financial Literacy and Retirement Income (previously Retirement Commission) for allowing me to add questions to the Financial Knowledge Survey, and to Colmar Brunton for data handling. For consistent support and encouragement, my supervisors Dr. Paul Callister and Dr. Richard Arnold, and from the School of Government Dr. Amanda Wolf and Dawn Yeabsley deserve many thanks. 
In preparing this thesis I have written articles based on short extracts. I would like to thank the reviewers, editors and publishers of these in the Longevity Bulletin (Institute and Faculty of Actuaries), the Journal of Population Ageing and the Institute of Policy Studies (IPS) monograph Retirement Income and Intergenerational Equity. Each of these articles was entirely my authorship based on my original research for this thesis. Previous work bearing my name referenced in this thesis was published by the British Actuarial Journal (BAJ). I conceived the idea for the BAJ paper and carried out 80 per cent of the writing and analysis with data collection by and joint authorship with Kim Dunstan. Of the Pensions Policy Institute (PPI) publications referenced here, three (PPI $2003,2004,2006)$ were written mainly by me with the final text reflecting input from the PPI team and independent reviewers. 


\section{Table of contents}

Chapter 1 : Introduction .......................................................................................1

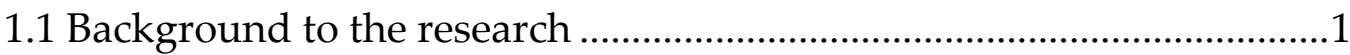

1.2 Research problem and hypothesis ......................................................

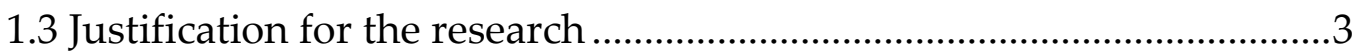

1.4 Methodology and outline of this report...............................................

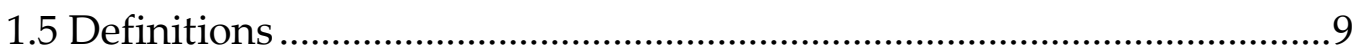

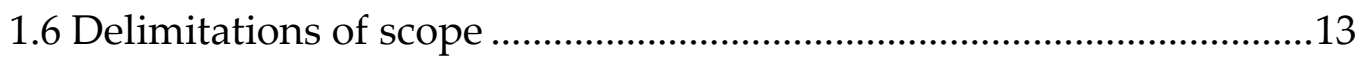

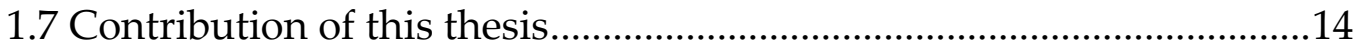

Chapter 2 : Literature review...............................................................17

2.1 Longevity risk in policy and retirement planning .............................17

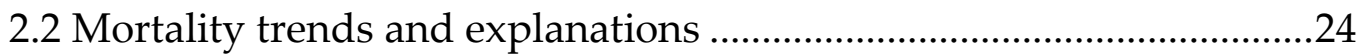

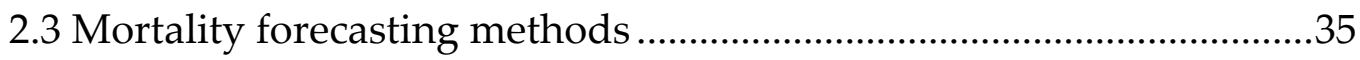

2.4 Uncertainty in mortality forecasting .............................................42

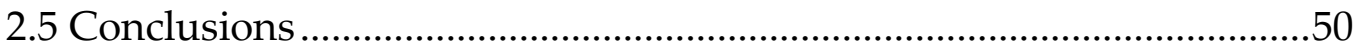

Chapter 3 : Theoretical basis for mortality analysis .............................53

3.1 Flaws in life expectancy measure ....................................................53

3.1.1 Period life expectancy underestimates real lifespans.................. 54

3.1.2 Misrepresented as individual lifespan expectation ..................... 57

3.1.3 Inadequate measure as mortality compresses ............................. 61 


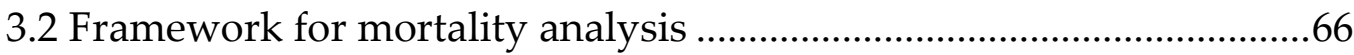

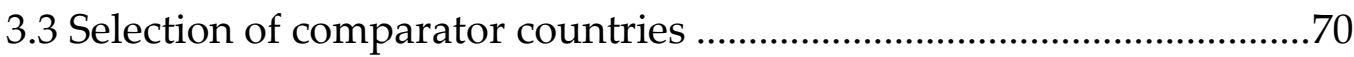

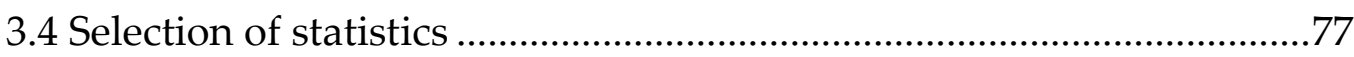

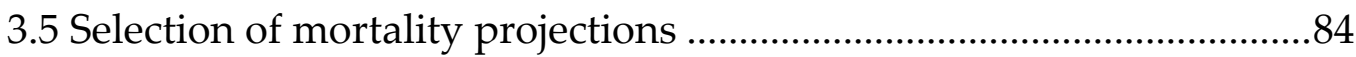

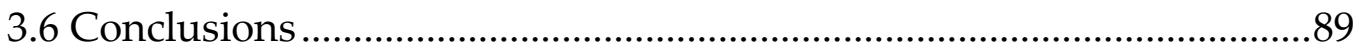

Chapter 4 : New Zealand mortality in international context ................91

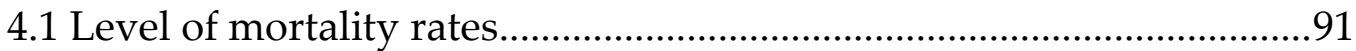

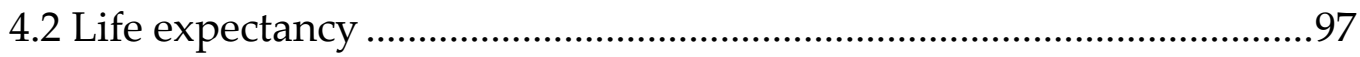

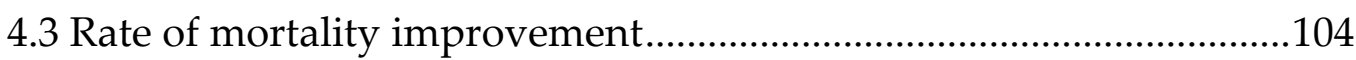

4.4 Mortality improvement by age and cohort ....................................107

4.5 Median and modal age at death....................................................127

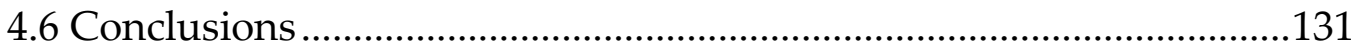

Chapter 5 : Future longevity in New Zealand .....................................135

5.1 Assumptions in international mortality projections ..........................136

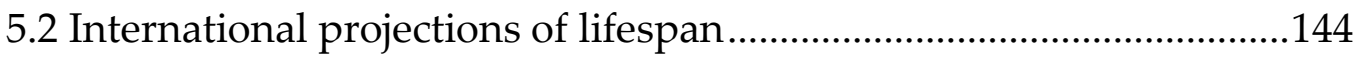

5.3 Reflecting New Zealand's relative mortality experience...................159

5.4 Impact of potential adverse trends ..................................................163

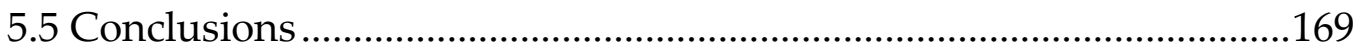

Chapter 6 : Mortality within New Zealand ..........................................173

6.1 Theories of mortality variation .........................................................174

6.2 Reflecting mortality of subgroups within New Zealand ...................180

6.2.1 Mortality data by subgroup ...................................................... 180

6.2.2 Māori and Non-Māori mortality................................................ 183

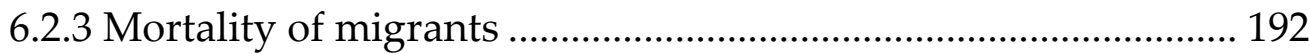


6.3 Variation in mortality: New Zealand compared ................................195

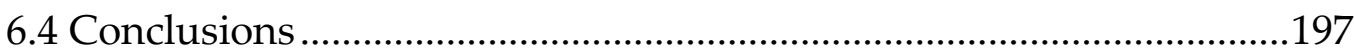

Chapter 7 : Surveying subjective lifespans.......................................201

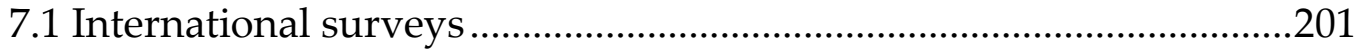

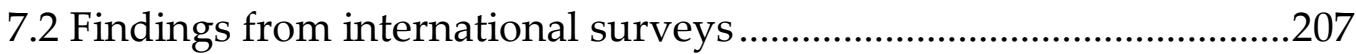

7.3 Structuring the research questions ..................................................218

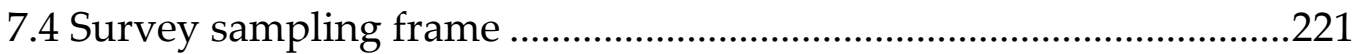

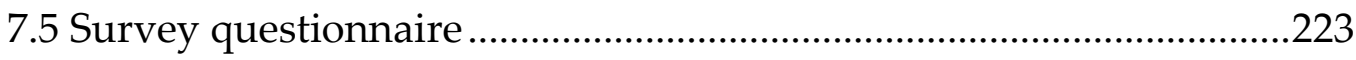

7.6 Survey questions on longevity expectations ...................................224

7.7 Choice of independent variables for analysis ................................2231

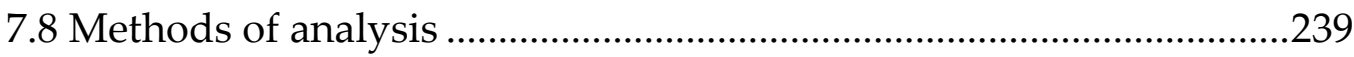

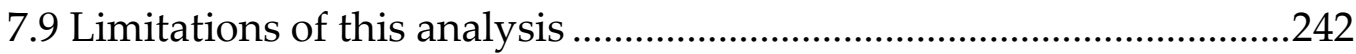

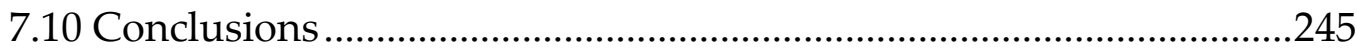

Chapter 8 : New Zealanders' lifespan expectations................................247

8.1 New Zealanders' subjective lifespan expectations (SLS)..................247

8.1.1 Distribution of SLS.............................................................. 247

8.1.2 Profile of SLS respondents....................................................... 251

8.2 How lifespan expectations are formed ...........................................255

8.2.1 How much New Zealanders have thought about lifespan ....... 255

8.2.2 How New Zealanders choose their SLS .................................... 261

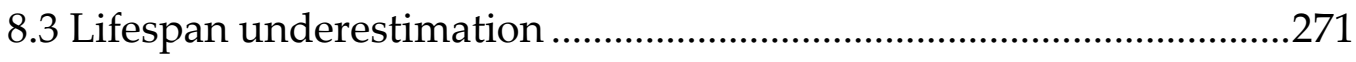

8.3.1 Predicting high or low SLS .................................................... 271

8.3.2 Over- and underestimation of SLS ............................................ 279 
Chapter 9 : Longevity risk in New Zealand

9.1 Potential for individual longevity risk ............................................295

9.1.1 How New Zealanders consider lifespan in retirement plans ... 296

9.1.2 Intended retirement age 297

9.1.3 Implied length of retirement .................................................... 302

9.2 Potential for longevity risk in public pension policy ..........................306

9.2.1 How lifespan is considered in pension policy ............................ 307

9.2.2 Longevity in New Zealand's superannuation policy ................. 317

9.3 Quantifying longevity risk in New Zealand ......................................321

9.3.1 Longevity risk in policy ......................................................... 321

9.3.2 Longevity risk in individual retirement plans ........................... 323

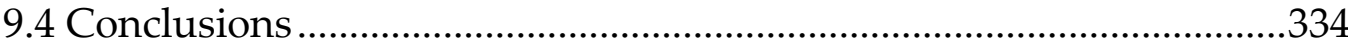

Chapter 10 : Conclusions and implications.......................................337

10.1 Longevity risk inherent and widespread .......................................338

10.2 Future mortality outlook in New Zealand positive ...........................340

10.3 New Zealanders underestimate likely lifespans .............................347

10.4 Lifespan indicators key to reducing longevity risk..........................353

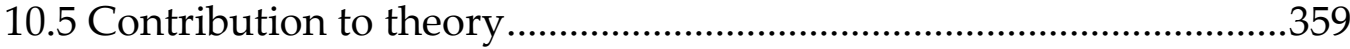

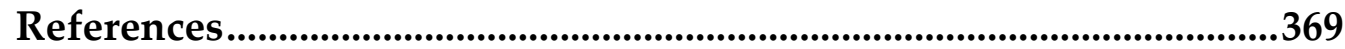




\section{List of figures}

Figure 2.1: Curve of deaths for females in the UK from three period tables (numbers dying at each age from 100,000 births experiencing age-specific mortality rates of year shown)

Figure 2.2: Survival curve for females in the UK from three period tables (numbers still alive at each age from 100,000 births experiencing age-specific mortality rates of year shown)

Figure 3.1: Curve of deaths from 100,000 births for females in New Zealand from three period tables, with mean, median and mode

Figure 4.1: Mortality rate at age $\mathrm{x}\left(\mathrm{q}_{\mathrm{x}}\right)$ for ages 0 to 100 years for selected countries, logarithmic scale, males, 1961

Figure 4.2: Mortality rate at age $\mathrm{x}\left(\mathrm{q}_{\mathrm{x}}\right)$ for ages 0 to 100 years for selected countries, logarithmic scale, males, 2006.

Figure 4.3: Mortality rate at age $\mathrm{x}\left(\mathrm{q}_{\mathrm{x}}\right)$ for ages 0 to 100 years for selected countries, logarithmic scale, females, 1961

Figure 4.4: Mortality rate at age $\mathrm{x}\left(\mathrm{q}_{\mathrm{x}}\right)$ for ages 0 to 100 years for selected countries, logarithmic scale, females, 2006

Figure 4.5: Period life expectancy at birth for selected countries, in years, 1961

Figure 4.6: Period life expectancy at birth for selected countries, in years, 2006

Figure 4.7: Average age-standardised annual improvement in mortality rates (QXI), 1961-2006, all ages to age 90, in five year age bands, males ... 108

Figure 4.8: Average age-standardised annual improvement in mortality rates (QXI), 1961-2006, all ages to age 90, in five year age bands, females 108

Figure 4.9: Curve of deaths for males from 100,000 births, from period table 1961, selected countries, ages 56 to 95 
Figure 4.10: Curve of deaths for males from 100,000 births, 2006, selected countries, ages 56 to 95 .

Figure 5.1: Cohort life expectancy at birth in calendar year 2010 for selected countries, main projection variants

Figure 5.2: Cohort life expectancy at birth in calendar year 2050 for selected countries, main projection variants

Figure 5.3: Cohort life expectancy at age 65 in calendar year 2010 for selected countries, main projection variants

Figure 5.4: Cohort life expectancy at age 65 in calendar year 2050 for selected countries, main projection variants

Figure 5.5: Cohort life expectancy at birth in years, by year of birth 1930 to 2050, for selected countries, medium projections, males

Figure 5.6: Cohort life expectancy at birth in years, by year of birth 1930 to 2050, for selected countries, medium projections, females

Figure 5.7: Cohort life expectancy at age 65 in years, by year of birth 1930 to 2050, for selected countries, medium projections, males

Figure 5.8: Cohort life expectancy at age 65 in years, by year of birth 1930 to 2050, for selected countries, medium projections, females

Figure 5.9: Age-standardised mean BMI in $\mathrm{kg} / \mathrm{m}^{2}$ by country in 2008 , with $95 \%$ uncertainty intervals, male (left) and female (right).

Figure 5.10: Age-standardised diabetes prevalence by country in 2008, with $95 \%$ uncertainty intervals, male (l) and female (r).

Figure 5.11: Age-standardised mean systolic blood pressure in $\mathrm{mmHg}$ by country in 2008, with 95\% uncertainty intervals, male (l) and female (r) ....... 166

Figure 5.12: Age-standardised mean serum total cholesterol in $\mathrm{mmol} / \mathrm{L}$ by country in 2008, with 95\% uncertainty intervals, male (l) and female (r) ....... 166

Figure 6.1: Mortality rate at age $\mathrm{x}\left(\mathrm{q}_{\mathrm{x}}\right)$ for ages 0 to 100 years by gender and ethnicity, logarithmic scale, New Zealand population, 2005-7.

Figure 6.2: Mortality rate at age $\mathrm{x}\left(\mathrm{q}_{\mathrm{x}}\right)$ for Māori as a percentage of that for nonMāori, ages 0 to 100 years by gender, New Zealand population, 2005-7..... 
Figure 6.3: Survival curve from 100,000 births for New Zealand from period tables 2005-7 for Māori and non-Māori, males and females

Figure 6.4: RLI measure for New Zealand length of life variation 1961-2003 196

Figure 8.1: What age do you think you will live to? Subjective lifespan, estimated percentage of the population by gender

Figure 8.2: Distribution of SLS responses compared to actual deaths in New Zealand in 2009

Figure 8.3: How much thought given to age might live to, estimated percentage of the population

Figure 8.4: What thought given to age might live to, estimated percentage of the population by age group..... 257

Figure 8.5: Number of reasons for why chose age will live to, estimated percentage of population

Figure 8.6: Main reason for choosing SLS, grouped, and predictors of being in each group

Figure 8.7: SLS, above and below median and extreme groups, estimated percentage of population

Figure 8.8: Estimated percentage of the population with SLS higher (overestimate), congruent or lower (underestimate) relative to population life tables

Figure 8.9: Gap between SLS and period life table, estimated percentage of the population by gender 283

Figure 8.10: Gap between SLS and Low Mortality cohort life table, estimated percentage of the population by gender 284

Figure 9.1: Likely retirement age, estimated percentage of the population.... 298

Figure 9.2: Implied length of retirement in years, estimated percentage of the population, grouped by tritile 


\section{List of tables}

Table 3.1: Examples of confusion in use of life expectancy measure 59

Table 3.2: Effects on measures of longevity of changes in mortality when mortality is generally improving

Table 3.3: Measures of central tendency for age at death, New Zealand females

Table 3.4: Population statistics for selected comparator countries

Table 3.5: Summary of available statistics which illustrate ethnic mix for selected countries

Table 3.6: Economic and life expectancy indicators for selected countries...... 75

Table 3.7: Main demographic and actuarial sources of national population projections in selected countries

Table 4.1: Directly standardised mortality ratio in year shown, standardised to New Zealand 2006 population 95

Table 4.2: New Zealand period life expectancy at birth, at age 65 and at age 80 compared to selected countries, 1961 and 2006. 100

Table 4.3: Average absolute deviation from average life expectancy at birth for the selected countries, 1961 and 2006, in years. 103

Table 4.4: Average age-standardised annual improvement in mortality (QXI) rates over decades and since 1961, all ages to age 90 105

Table 4.5: Average age-standardised annual improvement in mortality rates (QXI) by country and decade for five year age bands starting at age shown. 110 Table 4.6: Approximate birth years of golden cohort, by country and gender

Table 4.7: Approximate birth years of possible new young ages golden cohort, by country and gender.

Table 4.8: Improvement in life expectancy at birth, at age 65 and at age 80 in selected countries, 1961 to 2006, in years and per cent 
Table 4.9: Three measures of central tendency for the age at death distribution and the increase in years and per cent, 1961 to 2006, selected countries 130

Table 5.1: Summarised assumptions for the annual rate of change in mortality rates in population projections of selected countries

Table 5.2: Given rationale for the assumptions underlying population projections of selected countries

Table 5.3: Period life expectancy at birth and at age 65 for years 2010 and 2050 from medium mortality projections in selected countries, in years 145

Table 5.4: Increases in period life expectancy at birth and at age 65 from 2010 to 2050 from selected mortality projections, males and females, in years 147

Table 5.5: Excess of cohort life expectancy over period life expectancy at birth and at age 65 for years 2010 and 2050 from medium mortality projections in selected countries, in years

Table 5.6: Increases in cohort life expectancy at birth and at age 65 from 2010 to 2050 from selected mortality projections, males and females, in years 153

Table 6.1: Summary of mortality variance theories as they apply to comparing two subpopulations

Table 6.2: Age-standardised death rates by ethnic group, 1996-7 to 2005-7 ... 181

Table 6.3: Ethnic groups in New Zealand

Table 6.4: Numbers of deaths registered in New Zealand of New Zealand residents, by age and ethnicity, 2010

Table 6.5: Period life expectancy at selected ages, New Zealand Māori and nonMāori and the gap between them, 1960-62 and 2005-7 and change over that period.

Table 6.6: Percentage contribution to instantaneous change life expectancy at birth by age group, New Zealand 2005-7 by ethnic groups and gender. 189

Table 6.7: Variance in age at death at or above selected ages, New Zealand Māori and non-Māori and the gap between them, 1960-62 and 2005-7 ........... 190

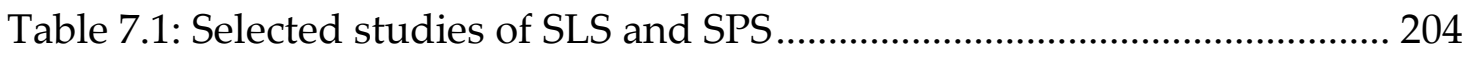


Table 7.2: Aggregate longevity expectations compared to population measures in selected studies

Table 7.3: Significant risk factors affecting direction of longevity expectations in selected studies

Table 7.4: Research objectives for survey of New Zealanders' longevity expectations

Table 7.5: Questions on longevity expectations in New Zealand survey........ 228

Table 7.6: Estimated sample errors, full sample, 95\% confidence level, assuming simple random sample.

Table 7.7: Demographic characteristics of sample

Table 7.8: Main single and dual ethnic groups

Table 7.9: Planning and financial knowledge characteristics of sample 238

Table 8.1: Summary of sample SLS compared to age-independent population lifespan indicators

Table 8.2: What age do you think you will live to? 253

Table 8.3: Have you thought about the age you might live to? 258

Table 8.4: Predictors of main reason for choice of SLS: multinomial logistic regression

Table 8.5: Predictors of SLS: binary logistic regressions

Table 8.6: Summary of gap between life table estimated lifespan (by age and gender) and SLS for men and women, in years

Table 8.7: Predictors of over- and underestimation of SLS compared to period life table: multinomial logistic regression

Table 8.8: Predictors of underestimation of SLS compared to Low Mortality cohort life table: binomial logistic regression

Table 9.1: Predictors of likely retirement age of 70 or more (including never), as compared with 65 or below 300

Table 9.2: Implied length of retirement, by tritile 304 
Table 9.3: Policy position on raising age of eligibility for the public pension in selected countries

Table 9.4: Longevity rationale invoked for proposed or actual increase in public pension eligibility age in selected countries

Table 9.5: Age at death assumptions that may be used by, or are available to New Zealanders.

Table 9.6: Sizing the effect of longevity risk on retirement plans. 
Underestimating lifespans? Why longevity risk exists

This page has been intentionally left blank. 


\section{Chapter 1: Introduction}

This thesis is about longevity risk: the risk that future lifespans are underestimated in superannuation (or pension) policy and individual retirement planning. It begins from a hypothesis that lifespans are routinely underestimated as mortality is improving, and investigates the extent to which that is the case, why it occurs, how significant is the consequent longevity risk and potential remedies. This is an internationally relevant subject, and international theory and evidence are used, with detailed findings developed in the case of New Zealand. This first chapter sets the context for the remainder of this thesis.

\subsection{Background to the research}

The 'ageing population' has been studied and commented on widely (United Nations 2007). Population ageing happens when the rate at which children are born decreases and lifespans increase as mortality rates decrease. This demographic change is occurring in all developed countries, including New Zealand (Dunstan and Thomson 2006). Population ageing has critical longterm implications in policy areas including superannuation, retirement planning and personal saving, health, housing and transport. Public policy making on these issues requires long-term future predictions on both the demand side - the numbers of older people claiming superannuation, for example - and the supply side: how many people will be contributing tax to pay for it. 
Generally for developed countries, including New Zealand, people are increasingly likely to survive to age 65 , the common age of eligibility for public pensions, and the average length of time an individual receives the pension is increasing. In the absence of policy change, improving mortality rates mean the cost of public pension rises. Policy making for issues of an ageing population therefore needs evidence on population mortality. Because mortality is improving rapidly, it is possible that assumptions about future mortality are conservative so that the assumed future average lifespans in the evidence base used by policy makers underestimate what is likely. In superannuation policy, this could result in higher fiscal cost than anticipated, a rushed decision to increase eligibility age when giving more notice would be preferred, or other necessary adjustments made more adverse than desired. Similarly, if individuals fail to realise that mortality is improving, they will not have considered the financial or other implications in their retirement planning, and will suffer longevity risk that will become apparent when their retirement appears too long for the amount of savings made. This thesis aims to fill knowledge gaps about longevity risk in New Zealand, noting features relevant more widely.

\subsection{Research problem and hypothesis}

The question posed in this thesis is: What is the extent of longevity risk in New Zealand superannuation policy and by New Zealanders; why does it exist; how significant is it; and, what are potential remedies?

The starting point that longevity risk is likely to exist is formed by the hypothesis that lifespans are routinely underestimated in this current era of improving mortality. Themes from a variety of source literature accumulate to 
suggest an inherent tendency to underestimate future longevity in both policy and individual settings. Further, it is common knowledge within demography and actuarial science that the period life expectancy indicator most often used to describe average lifespans is in fact flawed for that purpose, increasingly so at this stage of demographic change in developed countries, with the more technically correct cohort life expectancy indicator less likely to underestimate lifespan. Yet there has been little analysis of the practical consequences of this problem and none in relation to longevity risk. However, if misunderstanding of likely lifespans exists at least in part due to a faulty indicator of population average lifespan then public information about lifespan may play a role in causing longevity risk; and may therefore also be in part a remedy. This hypothesis further suggests that the two settings of policy and individual planning need to be considered together; as the first setting provides public information which may be used in the second.

\subsection{Justification for the research}

How long people live is of clear economic importance in superannuation policy. For individuals, their own (or others') potential lifespan may be an emotional subject, as well as being of financial consequence. An individual's estimate of their own lifespan has been suggested as a measure of the value of life itself (Ross and Mirowsky 2002).

Future lifespan is inherently uncertain, whether for an individual or a population average. There is only limited international evidence that comprises few full surveys of the expectations of lifespan among adult populations. Longevity risk in policy is increasingly of concern as mortality continues to improve and many developed countries face the fiscal pressures 
of ageing populations. That concern carries over to individual retirement planning as a response to those fiscal pressures is often for countries to adopt polices expecting more private saving for retirement.

Yet despite the importance of understanding the extent of longevity risk and its significance in policy and retirement planning settings, no studies have been identified which consider the extent of longevity risk in policy and by individuals throughout life. Identifying longevity risk requires the comparison of the lifespans used in the setting in question with likely future lifespans. In New Zealand, there has been no systematic review of any of these elements: the objective rationale for lifespans assumed in policy making; the empirical evidence for subjective lifespans used in individual retirement plans; or comprehensively justified plausible future population lifespans. This thesis seeks to fill the research gap in all three elements, and bring out conclusions for the extent and nature of longevity risk in New Zealand.

Furthermore, the analysis in this thesis develops existing theory with new emphasis. Because this study is interested in how individuals' expectations of longevity collectively compare with population benchmarks used in policy making, it emphasises individual lifespan measures. This approach has received little attention, as most analytic work on mortality is concerned with the population average. Average measures are often not sufficient for policy decisions, so this study also integrates emerging demographic theories on variance in lifespan to consider the distribution of individual lifespans and subjective lifespans. 
Finally, the addition of actuarial literature and techniques to the demographic approach usually used in New Zealand supplements the evidence base and allows new interpretations. The theoretical framework for this research is a specific subject - longevity, or its counter-cause, mortality which is situated in both demographic and actuarial literature. Demography, a distinct discipline within social science, uses data "as shrewdly as possible" to explain observed trends in vital human processes (Caldwell 1996 p. 333). Demography has a "small phenomenological turf" (Preston 1993 p. 603), being usually populations of national or regional size. Actuarial science has an even smaller turf of life insurance and annuity populations of commercial interest, but has developed specifically from the consideration of future mortality and longevity risk (Haberman 1995). Actuarial science uses many of the same techniques as demography and actuarial concepts are applicable to national populations. However, the integration of demographic and actuarial literature is limited in New Zealand compared to practice in some other countries. This research uses the knowledge and theory of mortality from both international disciplines, drawing a greater input from actuarial science than has been the case in New Zealand population studies.

\subsection{Methodology and outline of this report}

Answering the research question posed in this thesis requires a number of different investigations in two main settings - policy and individual retirement planning - which have different literatures. It sources demographic data from five countries. It accumulates the results of different empirical analyses which have been developed from established and emerging demographic and actuarial techniques. Because the research question has not been asked before, 
the methodology, or rather, collection of methods, has had to be uniquely determined.

Chapter 2 provides a review of existing literature on: longevity risk in policy and retirement planning; mortality trends and explanations of those trends; mortality forecasting methods; and uncertainty in mortality forecasting. It accumulates the evidence for a hypothesis that assumed future lifespans are likely to underestimate likely lifespans.

In order to develop a view on likely future lifespans in New Zealand, a number of approaches could have been taken. Before this thesis the results of only limited testing of the plausibility of the mortality assumptions underlying Statistics New Zealand's population projections was available. Chapter 3 develops theories of mortality to describe the methodology chosen to assess a plausible future for New Zealand's likely future longevity, and the rationale for the choice. The method developed in this thesis takes a macro view of population mortality trends rather than a micro view of single risks to mortality, and supplements existing extrapolative demographic projections with analytic techniques drawn from actuarial science. A new framework on which to make a qualitative assessment of the plausibility of the mortality assumptions in official projections is developed using: a quantitative international comparison of past mortality trends; a quantitative international comparison of projections of future mortality; and, a mostly qualitative investigation into the mortality of subgroups within the New Zealand population. 
The next three chapters describe the results of each of these analyses. In Chapter 4, the past mortality trends of New Zealand are compared with those of the selected comparator countries using a comprehensive range of mortality measures. This enables the comparison of future longevity projections of the same countries to be tested for consistency, and conclusions drawn about the relative optimism or pessimism of the assumptions made. This analysis is set out in Chapter 5. In Chapter 6, the variation in mortality between subgroups within the New Zealand population is examined. That chapter also describes how emerging demographic theory on variance of mortality is developed to supplement the preceding analysis based on population average mortality.

The lifespan assumptions used by individual New Zealanders in their own retirement planning required an original empirical study. A survey was carried out by adding questions to a planned survey of New Zealanders' financial knowledge undertaken by the Commission for Financial Literacy and Retirement Income (then called the Retirement Commission) which provided the context of retirement planning. The questions added to identify individual expected ages at death (or "subjective lifespan") were developed having learned from similar studies in other countries. This survey additionally extended the scope of questions in order to identify the reasons why respondents chose a particular subjective lifespan. This revealed insights to address longevity risk causes and remedies. The development of the survey approach is set out in Chapter 7.

The results of the survey on subjective lifespan are shown in Chapter 8 . The analysis of the responses was developed in order to describe the extent of under- or overestimation of lifespans, compared to a range of possible outcomes represented by different projections from Statistics New Zealand. 
This technique was more detailed than that used in some previous studies and developed a more technically correct assessment of the distribution of lifespan underestimation.

The results of the preceding analysis were used to quantify the extent of longevity risk in New Zealanders' retirement planning and New Zealand superannuation policy. Chapter 9 integrates the findings in earlier chapters, focusing first on individual retirement planning, including some further results on retirement planning behaviour from the survey, secondly on policy and thirdly drawing out the links between the two settings. Once the inputs had been developed the calculation was straightforward. For the individual setting, it used an existing planning tool, available to all New Zealanders, which follows the theory of the lifecycle savings hypothesis. For the policy setting, existing Treasury fiscal projections were able to be used. Chapter 10 concludes and discusses wider policy and research implications. 


\subsection{Definitions}

The mortality rate, a basic unit of demographic or actuarial analysis, is defined as the probability of dying at a specific age at a specific time. In demography, the term death rate is generally used instead. A mortality rate can be an assumption for, or can represent the actual experience of, a specified population at a specified time. In actuarial notation, the initial mortality rate at age $x$ is represented by $q_{x}$, which is the number of deaths at age $x$ divided by the number of people who had their xth birthday in a certain period. Another type of mortality rate, $m_{x}$, or crude death rate, represents the number of deaths aged $\mathrm{x}$ in any year divided by the population of that age at the middle of the year.

The relationship between the two rates:

$$
q_{x}=\frac{\mathrm{m}_{\mathrm{x}}}{\left(1+0.5 \mathrm{~m}_{\mathrm{x}}\right)}
$$

holds at all but ages less than one year (Hinde 2007). For the purposes of this thesis, the difference between $q_{x}$ and $m_{x}$ is not significant, and the term 'mortality rate' will be taken to imply the concept of the probability of dying at age $x$, population and time to be specified. Detail on whether q-type or m-type mortality rates are used is given where necessary in the text.

Life expectancy is calculated from mortality rates for a defined population. It is a summary metric representing the average number of years left to live for members of a defined population at a specific age. Life expectancy at age $\mathrm{x}$ is represented in actuarial notation as ex. A fuller explanation of this measure and its use is in Chapter 3. Table 9.5 provides a number of life expectancy or alternative measures that may be used by, or are available to New Zealanders. 
Lifespan is a measurable unit; the age at death for an individual. Lifespan as applied to a population or species requires a modifier, for example average length of life for a cohort born in a specified year (Carey 2003). The maximum observed lifespan for humans is 122 years (Robine and Allard 1999).

Longevity is a general term used here to indicate long life.

Longevity risk is defined as per Stallard (2006) as the possibility that future lifespans turn out longer than anticipated, that is, assumptions of future lifespans are underestimates. More detail is in section 2.1.

Low Mortality, Medium Mortality (the "central estimate" or "medium estimate") and High Mortality refer to the different assumptions for future mortality underlying variant total population projections produced by Statistics New Zealand. Primarily, the 2009 (base)-2061 projections are used as these are the latest National Population Projections as at the date of this thesis. The mortality assumptions and results are referred to as "official mortality projections", although Statistics New Zealand does not use this term. Chapter 5 sets out in detail the mortality assumptions and the resulting life expectancy and other measures for selected countries. Very High Life Expectancy refers to an additional variant projection of Statistics New Zealand which assumes period life expectancy at birth reaches 95.0 years for the New Zealand population in 2061.

Mortality risk is used here to refer to an external factor or characteristic of an individual or population which increases a mortality rate. 
Superannuation is used interchangeably with public pension, or state pension, that is, the income benefit provided by a Government for its citizens, residents or others eligible once they reach a defined age. In New Zealand, the public pension is called New Zealand Superannuation (NZS).

Pension age or age of eligibility is the age at which full benefits of the public pension first become available. The age of eligibility for New Zealand Superannuation is age 65.

Defined Benefit (DB) and Defined Contribution (DC) refer to two types of private pension scheme. In DB schemes the regular pension benefit to be received is defined by reference to a fixed parameter, such as a percentage of final salary. In DC schemes the pension benefit is determined at the point of first receipt. The benefit will depend on the investment returns achieved on the contributions made and on the terms of any annuity or other product used to turn the accumulated contributions into income.

KiwiSaver is the main retirement savings product in New Zealand. It is a voluntary, work-based, regulated savings initiative, sponsored by the government with schemes provided by private managers. It is a DC product. New Zealanders are automatically enrolled into KiwiSaver when they start a new job, but may opt out, and may join at any time.

The Retirement Commission changed its name to the Commission for Financial Literacy and Retirement Income in October 2011. The Commission is an autonomous Crown Entity responsible for financial education in New Zealand and hosts the Sorted financial information website (www.sorted.org.nz). The Retirement Commissioner (this title did not change) 
publishes a review of retirement income policy every 3 years. This thesis generally uses the abbreviated name of the Commission, CFLRI, but references the Retirement Commissioner's reports under that title.

UK refers to the United Kingdom of Great Britain and Northern Ireland. "British" is used as the adjective for data from this entity. Where data is sourced from Great Britain only, it will be referred to as "GB". For historical consistency, some data used will be sourced from England and Wales (E\&W) the population of which is 88 per cent of that in the UK (ONS data).

Other abbreviations used in this thesis include:

NZ: New Zealand. US: United States of America.

$\mathbf{M}$ or $\mathbf{m}$ : Male. $\mathbf{F}$ or $\mathbf{f}$ : Female.

QXI: Annual rate of improvement in the mortality rate (see section 3.4).

ONS: Office for National Statistics, UK; SNZ: Statistics New Zealand.

In Chapter 7, variables of individuals' view of their own life expectations are defined:

SLE: Subjective life expectancy.

SLS: Subjective lifespan.

SPS: Selective probability of survival 


\subsection{Delimitations of scope}

This study considers two domains: public pension policy and individual retirement planning. Retirement planning is used here to mean financial planning and saving for retirement. It excludes other personal considerations that may be related to retirement such as housing, transport or care. The policy considerations of these issues are also excluded.

This study is not concerned with longevity risk for a private provider of insurance, pension or annuity products. The mortality data considered is that of national populations, not the populations within portfolios of such providers. Similarly, when longevity risk of an individual is considered, it is assumed that the individual is representative of any member drawn at random from a national population, not a policyholder or scheme member of an insurance or pension provider. Individual underwriting by an insurance or pension provider is not considered here. Issues relating to specific risk to the individual or the provider (such as moral hazard) are not considered. Annuitisation or other options for drawing down accumulated retirement savings are excluded. This analysis investigates individuals' exposure to longevity risk pre-retirement, being concerned with the risk of not saving enough for later longevity (see section 2.1).

In this thesis, the expectations that adults have for their lifespan are measured against population indicators. This allows the consideration of the collective tendency for expectations to vary from the parameters used in policy making. The accuracy of individual predictions for age at death is not considered: that would only be known for an individual after death. 
This thesis is about mortality and lifespan - how long life is. The quality of life, which may be measured by healthy life expectancy or other indicators, is important for both policy and individuals, but is out of scope for this thesis. Further, the measures used for lifespan here are generally measures of central tendency in national populations. Consideration of the variance in lifespan is made in Chapter 6. The commentary on variance of lifespan is restricted to what is needed to explain how variance is theoretically relevant to the analytic framework developed, with some empirical data from New Zealand. This should not be interpreted as a full analysis necessary to understand variance or inequalities in lifespan between or within countries.

\subsection{Contribution of this thesis}

This thesis explores the existence, causation and remedies for longevity risk. Previous literature defines longevity risk, but any further investigation has been mainly confined to commercial applications. This thesis considers longevity risk in two under-explored domains: individual retirement planning and public pension policy. In doing so, it contributes to theory and to empirical data in four main ways.

First, this research takes a new theoretical perspective on measures of lifespan. Unusually, this research takes the perspective of individual lifespans rather than using the more common analysis framework of a whole population. The starting point is the comparison of likely lifespans with the lifespan expectations of individual New Zealanders, so longevity risk in the individual domain is explored. This leads to a consideration of the potential 
influence that indicators used in policy have on individuals, enabling an exploration of longevity risk in the policy making domain.

Second, this thesis extends existing theory and practice to undertake original research on past mortality and lifespan prospects in New Zealand. This research adds new theoretically-based insights to the relatively limited mortality analysis so far undertaken of trends within New Zealand. This approach emphasises learning from trends in like countries, which enables conclusions to be made about the plausibility of different variants of the existing New Zealand population longevity projections. The design of the analytical framework used here references techniques from both demography and actuarial science, where usually analysis exists within a single discipline. The framework could be used for other national populations. The framework is unusual for focusing on few peer countries for international comparison but using a wide range of different mortality indicators: the population average level and rate of change of mortality rates and life expectancy; recent past, current and future projected mortality indicators; and, a consideration of variance in mortality within the country in question. This thesis therefore contributes an empirical database and interpretation of a range of mortality indicators for Australia, Canada, UK, US and with greater detail for New Zealand.

Third, this thesis builds on existing research on subjective lifespan expectations in a new setting. It contributes the first set of empirical data on the lifespan expectations of individual adult New Zealanders. The survey instrument and sample also contribute the first results from a national adult population about reasons for choice of subjective lifespan and retirement age intentions, and the associations of these with lifespan expectations and a full 
range of demographic variables. The methodology improves technically on previous studies to allow the calculation of the distribution across the adult New Zealand population of the gap between individual expectations and lifespan outcomes defined by official estimates. Individuals' reasons for their lifespan expectations are probed for the first time.

Finally, this research accumulates theory and empirical findings to build a unique collection of analyses targeted at a specific research question not previously considered. This analysis specifically questions why longevity risk exists in the pre-retirement phase of New Zealand's superannuation policy and individual retirement planning and enables some quantification of longevity risk under current policy settings and longevity prospects. The research method illustrated here to diagnose longevity risk in New Zealand would be applicable elsewhere. The remedies suggested, based on the evidence on longevity trends, are a contribution to the public framework for individuals' retirement planning and to the policy debate on the age of eligibility for superannuation. 


\section{Chapter 2: Literature review}

How long each of us will live is unknown. The inherent uncertainty engages individuals emotionally and has consequences which have captured the attention of policy makers globally. Statistics on mortality have been analysed in great detail and many theories developed to explain the drivers of lengthening lifespans. The literature on which any exploration of longevity and its consequences is based is therefore large and encompasses a range of disciplines.

This chapter reviews theory and literature on mortality and longevity which form the starting point for exploration of longevity risk: the hypothesis that assumptions of future lifespans are likely to underestimate. The first section explores what is known about longevity risk in both policy making and individual retirement planning. The next section discusses the history of mortality trends and the explanations for them. The third section describes mortality forecasting techniques to set the context for the fourth section which discusses how well mortality forecasting deals with the inherent uncertainty about future trends. Each of these sections takes a global view before discussing the New Zealand situation.

\subsection{Longevity risk in policy and retirement planning}

Given uncertainty about how long each individual will live, future longevity can be over- or underestimated in national projections or forecasts and in public messages about longevity, as well by individuals. This thesis takes the 
position, established by the definition of longevity risk, that underestimating longevity is more serious than overestimating.

"Longevity risk can be defined at individual and aggregate levels. At the individual level, longevity risk refers to the possibility of living longer than assumed in financial planning for the retirement of a single individual. At the aggregate level, longevity risk refers to the possibility of a higher average number of years of survival than assumed in designing a retirement security system for the aggregate."

Stallard (2006 p. 575)

In the context of personal retirement planning, the risk in overestimating longevity is saving too much for a retirement that turns out to be shorter than expected, potentially missing out on lifetime consumption as a result. The risk of underestimating longevity is failing to provide financially for all of the years of retirement an individual turns out to have. Longevity risk as defined asserts that the latter is a more significant risk. At the aggregate population level, in the case of national public pension policy, the risk of overestimating future longevity is virtually cost-free, as most public pension benefits are not funded in advance. As it became apparent that longevity was worse than expected, less public money would be needed to be spent on pensions than expected. However, if in setting the parameters of the public pension, policy makers underestimate potential future lifespans, the consequent risk is that pension payments assumed to be promised have to be unexpectedly cut back or restricted; or other government spending compromised. Thus, the concern here at both the individual and national population levels is longevity risk as defined by underestimating longevity. 
The impact and risks of increasing longevity in public pensions has been a policy area of intense interest in the last decade or more. The World Bank first drew attention to the need to consider pension reform because of the ageing population and greater longevity in its seminal 1994 report Averting the Old Age Crisis. Since then, the topic has continued to be followed by major international organisations (Barr 2000; Holzmann and Stiglitz 2001; OECD 2011b; United Nations 2007; World Bank 1994).

Pension reform has multiple strands but a feature shared by many countries is an increase in the age at which the public pension first becomes payable. The OECD reported in 2007 that nearly all its 30 member countries made some reforms to pension systems since the early 1990s, 16 of them designated "major". The reform issues outside the scope of this study include how the level of average pension income compares with pre-retirement income, disparities in pension income levels, the balance between public and private provision and the nature of financial risks in private pensions. However, the OECD reported that an increase in pension age (the generic term for what in New Zealand is called age of eligibility for superannuation) was the most common feature of reform packages (OECD 2007, 2011b).

In many ways, the focus on increasing the age of eligibility is not surprising. Actuaries and demographers, working from their knowledge of lengthening lifespans as mortality improves, often make the intuitive leap to raising eligibility age as a policy response (Sanderson and Scherbov 2007; The Actuarial Profession 2006). The cost of providing public pension or superannuation in future can be reduced dramatically when eligibility age is increased (DWP 2010; Rodway 2010). 
Acting against this theoretical enthusiasm, there are concerns about the fairness of increasing eligibility age when mortality varies across a population and political concern about the unpopularity of such a plan (PPI 2003). Commentators on superannuation have explored ways to overcome these problems, for example, by having a lower eligibility age for workers with relatively low lifetime earnings (Zhivan et al. 2008) or by making the increase in eligibility age automatic by indexing to longevity data (Shoven and Goda 2008). No research was found that assessed and compared the process of decision making or the mortality or longevity data used in decisions about eligibility age. The decisions may have been too hasty if mortality improvement is set to slow, or conversely longevity risk could exist even in countries raising the age quickly if the reform was rationalised using evidence which underestimates longevity.

Longevity risk for individuals is most often discussed in the context of annuitisation under the framework of the lifecycle savings hypothesis which posits that people build up savings pre-retirement then draw down those savings after retirement (Brown 2003). Annuities are a specific method of draw down: by using the accumulated savings to purchase an annuity, the purchaser is promised a regular income for life, and longevity risk is passed from the purchaser to the annuity provider. The puzzle of why few people voluntarily buy annuities when the lifecycle savings hypothesis would predict it is the most economically rational action has preoccupied economists for some time (for example Brown 2001). The increasing interest in this area of policy research has been stimulated in part by the growth in ownership of defined contribution (DC) private pensions and the decline in membership of defined benefit (DB) pension schemes. DB schemes hold the longevity risk 
within the scheme, but DC pensions are individual accounts where longevity risk is held by the individual. Although New Zealand has had a smaller membership of DC pensions than other countries, it has also debated annuitisation or other drawdown options (Retirement Commissioner 2007, 2010). The relevance to New Zealand of post-retirement longevity risk for individuals increased following the introduction of KiwiSaver in 2007, as money invested in KiwiSaver is intended to be accessed as a lump sum at the age of eligibility for superannuation.

In New Zealand and other countries then, the focus of individual longevity risk in literature and policy discussion has been in the postretirement period. Although the effect of longevity risk is felt after retirement, when money saved is proven to be less than needed to last for a later than expected death unless annuitised, a contributory cause is not saving enough during the pre-retirement period, or by giving up work sooner than proved ideal. A whole life perspective to longevity risk is therefore important. Issues around annuitisation or other product options in the post-retirement period do not provide a complete explanation of longevity risk.

The issue of building up enough money in retirement savings has also received some attention, but the focus here has been whether people are saving enough for a particular retirement income goal. Such analyses have been made for example in the UK, USA and New Zealand (Engen et al. 2001; Pensions Commission 2004, 2005; Scobie et al. 2006). All are based on similar methodologies, again based on the lifecycle savings hypothesis: they assume a target retirement income, project forward what pension individuals representative of the population will have from the state, calculate how much saving will make up the difference to the target, and then compare that so- 
called 'required' amount of saving with the actual level of saving observed. All these analyses find some evidence of undersaving, but all are complex calculations, with controversial assumptions (see PPI 2006, pp. 12-14). However, the risk being considered is that savings made are not sufficient given an assumed lifespan. The risk that the assumed lifespan is underestimated has not been considered in studies of pre-retirement savings behaviour.

This thesis is primarily concerned with the longevity risk individuals face because they underestimate their potential longevity before retirement. If they underestimate then, whether or not they plan explicitly, they might: have a longer retirement than their lump sum or annuitised savings will cover; be reliant on the public pension alone for longer than intended; or, give up paid work earlier than they ideally should have done. Previous work based on the lifecycle savings hypothesis focuses on economic outcomes assuming expectations about longevity were met. The issue of concern in this thesis is instead the economic risk of expectations about longevity not being met.

In the context of retirement planning and pension policy, individuals' longevity expectations, together with their expectations on retirement age, present an implicit assumption about how long superannuation and other retirement income have to last. The realism of individuals' expectations of their own longevity could be a marker of how thoughtful individuals are about planning for their retirement. The value people place on the personal longevity they expect is thought to influence directly individuals' economic decisions including retirement choices (Hamermesh 1985). Collectively, individuals' assessment of lifespan may also indicate how receptive the public is to policy reforms based on rationale about trends in longevity, such as 
increasing eligibility age. If there is a widely-held understanding that mortality is generally improving then an increase in eligibility age is likely to appear more reasonable than if the public believe average lifespan is stable.

It has been suggested that how long an individual expects to live is a measure of the value of life. It "assesses one's store of the essential property existence itself" (Ross and Mirowsky 2002 p. 470). However, despite such claims for the importance of such expectations, there are few surveys representative of national adult populations providing empirical evidence for what these expectations are. The methodology and results of these international studies are examined more closely in Chapter 7. In brief, although summary findings as reported in these studies suggest that subjective lifespans are close to lifespans predicted in population life tables, this hides both over- and underestimation of longevity.

The study that appears to be most relevant to an investigation of a national population suggests that the tendency is to underestimate lifespans because people ignore expected mortality improvements. This study of the adult population of Great Britain found an average underestimation of lifespans of over four years for males and around six years for females compared to plausible contemporary estimates of future lifespans (O'Brien et al. 2005). A theme from this and other studies (notably Hurd and McGarry 1995, 2002; Mirowsky 1999; Popham and Mitchell 2007) is that men reflect that they have lower actual longevity than women on average, but men are more optimistic, so that women are more likely to underestimate their actual longevity. However, studies have not probed how people think about longevity, if they think about it at all, whether or how they take individual risk factors into account or rely on a notion of the average, or how and why they 
choose a particular estimate of their own likely longevity when asked. Therefore, international evidence suggests a risk of some significant underestimation of future lifespans, but offers little explanation for the mechanism by which it occurs. The role expectations play in longevity risk is therefore unexplored, but could be significant both for individual retirement planning and in policy.

\subsection{Mortality trends and explanations}

Mortality has been improving in nearly all countries, including New Zealand, and as a result average population lifespans have become longer. This section reviews the literature on the trends in historic mortality rates and the explanations for the factors driving the trends.

Past mortality trends have been and continue to be measured for specific purposes such as the impact of public health measures on deaths from a specific cause. Similarly, historic population mortality trends are analysed for insight into what has affected observed trends in longevity. In this thesis, which is concerned with estimates of future lifespans, past mortality trends are interesting insofar as they contribute to theories about the possible future patterns of population longevity. It is well established in demography that understanding past mortality trends is an important part of theorising future trends (for example, Hajnal 1955).

The history of mortality rates in the western world is overwhelmingly one of improvement. Mortality rates for adults in middle and older ages fell from the 17 th century onwards even without specific planning for such a result (Peller 1948). Late 19th and early 20th century interventions to improve 
medical care, sanitation and living environments resulted in reductions in infectious diseases and lower mortality rates particularly for infants and children. The pace of improvement in mortality rates at middle to older ages increased towards the end of the 20th century. Mortality rates were generally stable from the 1950 s to the 1980 s as motor vehicle accidents and smokingrelated deaths balanced out medical advances. But after the 1980s, public health initiatives were effective at changing behaviours especially in the wearing of seat belts and reductions in smoking. Medical science developed still further. By the end of the 20th century, diseases of middle to older life (cardiovascular, cerebrovascular and cancer) could be treated and lifespans extended longer than previously predicted (Preston 1996).

These changes in the level of age-specific mortality rates over time are often shown by means of the 'curve of deaths' - the numbers of life table deaths at each age from a starting number of births. Figure 2.1 shows this for females in the United Kingdom over a time period selected from available consistent data to illustrate the last eighty years of history outlined above. 
Figure 2.1: Curve of deaths for females in the UK from three period tables (numbers dying at each age from 100,000 births experiencing age-specific mortality rates of year shown)

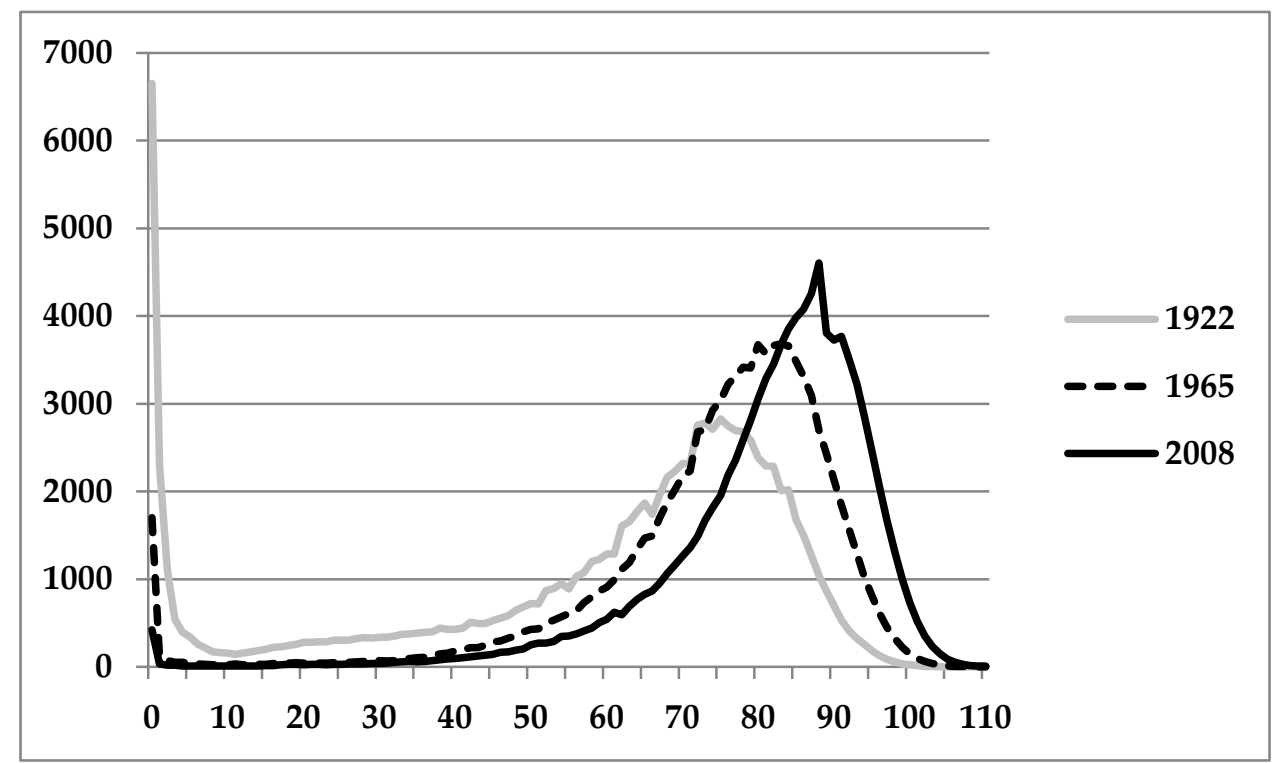

Source: Period life tables in the Human Mortality Database, November 2010 revision.

The curve of deaths is asymmetric with two modes. The reduction of the first peak at age zero has been significant even from the early 1920s. The second mode is at adult ages of death. In the last half century especially this mode has shifted to the right, a process called mortality shifting. A narrowing of the peak is also visible. This is due to improved health survival to older ages, halted by slower progress in maximum lifespan. The latter trend is called compression of mortality by some authors or the rectangularisation of the survival curve as the alternative presentation in Figure 2.2 demonstrates (for example Fries 1980). 
Figure 2.2: Survival curve for females in the UK from three period tables (numbers still alive at each age from 100,000 births experiencing age-specific mortality rates of year shown)

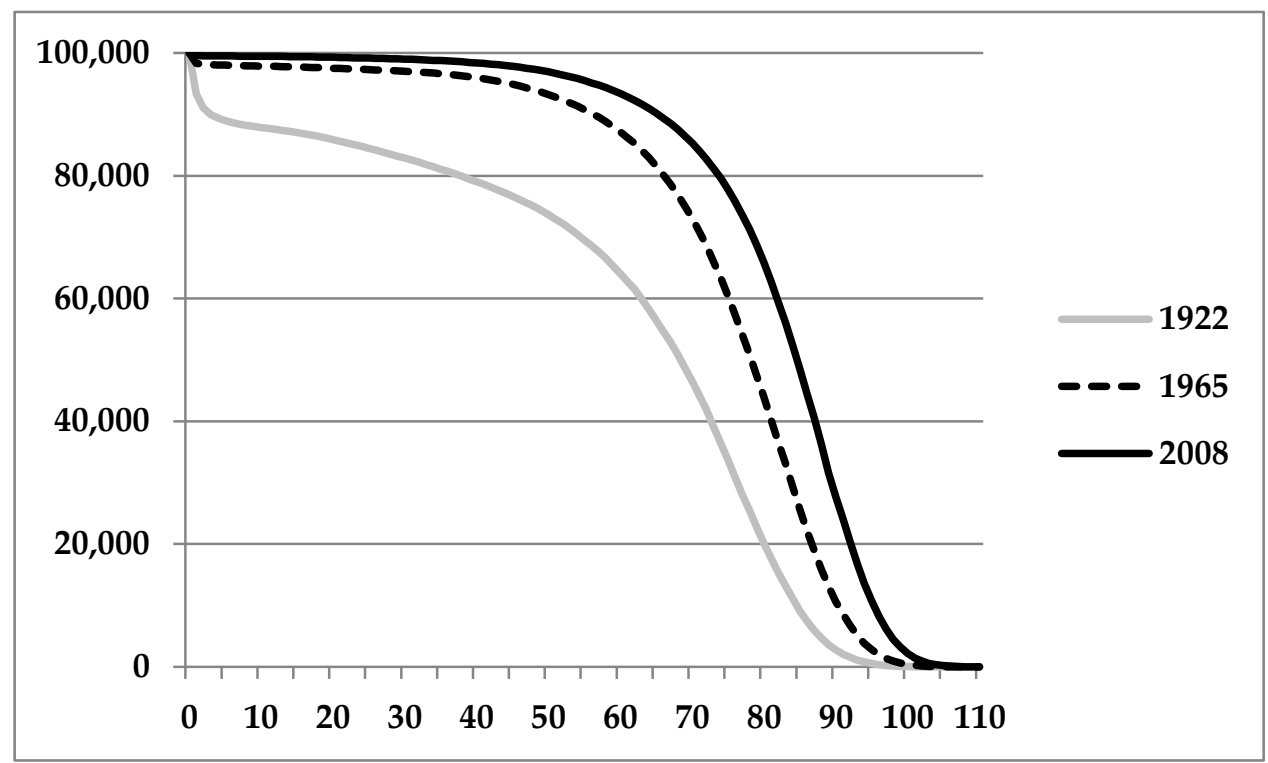

Source: Period life tables in the Human Mortality Database, November 2010 revision.

In the last few decades, there has been a significant, and unexpected, fast improvement in mortality at older ages so that we are now in the era called "delayed ageing" by Kannistö (2001), and described as follows:

"...mortality in old age [ages 80 and over] has undergone in the developed countries during the post-war period a deep-going and fairly general transformation reaching much lower levels than have ever been recorded before. Unprecedented in known demographic history, this decline has made in the prevailing trend a break which had not been foreseen in population projections."

Kannistö (1994) Chapter 15 
As hinted in the above, the pace of improvement in mortality rates after the 1980s, especially at older ages, came as a surprise. Despite now understanding better the past improvement in older age mortality rates, there is no suggestion that uncertainty in forecasting the future from the present is diminishing. If anything, current understanding of the factors influencing mortality has increased the potential for theories on mortality to be developed, but the complexity of those theories means there is still a great deal of uncertainty about how mortality trends will develop in the short and long term (Vaupel 2009).

Age and gender have been the usual two determinants in actuarial tables but it is now understood that there are many other factors influencing mortality rates. Harper and Howse (2008) have summarised these factors. Their summary provides the basis for the following list of the main groups of factors influencing mortality, with supplemental references as shown:

- Socio-demographic factors including age, gender, ethnicity, country of origin and marital status. All other things being equal, mortality rates increase with age and are lower for females than males. Married people have lower mortality rates than never married, divorced or widowed. The relative mortality of ethnic and migrant groups depends on situation. In New Zealand, Māori and Pacific people are known to have a higher relative risk of dying, and Asian residents a lower risk of dying, than the New Zealand population not in those ethnic groups (Jatrana and Blakely 2008; Statistics New Zealand 2009c). 
- Socio-economic factors are expressed differently in different countries, with income or education sometimes used as a proxy. Mortality rates worsen as socio-economic status worsens, even within small populations (Marmot 2005). A large body of research on mortality variance, and its association with how finance, education, housing, employment, transport, and healthcare are distributed exists in the fields of epidemiology and public health (for example, WHO 2008). However, the mechanism by which individual factors or socioeconomic status however defined affect mortality risk is not well understood. The evidence that education acts directly to improve mortality independent of socio-economic status is said to be underappreciated outside of demography (Baker et al. 2011).

- Life course factors are usually explained by the 'developmental origins theory'. This posits that conditions in utero, at birth or in very early childhood influence an individual's mortality risk even at advanced ages (Barker 2007; Eriksson 2005). The potential importance of early life influences is supported by observation of birth cohorts who exhibit a characteristic mortality trend within a population, although early life factors may not be a complete explanation for the trend. For example, the 'golden cohort' born between 1923 and 1940 in the UK has consistently experienced more rapid improvement in mortality rates throughout life than those born before or after (Dunnell 2008; Willets 2004). 
- Lifestyle factors are to do with personal behaviours. In most developed countries, clear evidence has been found for higher mortality rates, all other things being equal, for those who smoke, eat an unhealthy diet, are obese, take inadequate exercise, drink excessive alcohol, or participate in hazardous sports or other risky behaviour.

- Genetic disposition for longevity. In demography, studies have shown a clustering of long lifespans in families, suggesting a genetic component to exceptional longevity (e.g., Willcox et al. 2006). To understand fully the genetic basis for longevity needs large-scale and logistically complicated studies. To date, many genes potentially implicated in ageing have been identified, but few confirmed by multiple findings (Christensen et al. 2006; Fallin and Matteini 2009).

- Medical technology, including the use of preventative pharmaceuticals such as statins to lower blood cholesterol, has significantly contributed to the lowering of mortality rates for certain diseases. Carey (2003) suggests that the biological future of human lifespan will be determined by lifestyle ("healthful living") and three other factors, all in the field of medical technology: disease prevention and cure, organ replacement and repair, and ageing arrest and rejuvenation science.

These factors are individually complex. In some cases the pathway by which a factor might affect health or mortality is not understood. It is not understood how these factors work together to produce the overall mortality risk for any individual at a certain age. In particular, the relative importance of early and later life factors remains unresolved (Vaupel 2009). Factors suggested to explain the existence of the golden cohort in the UK cover the 
lifecourse, including improvements in the UK's ante-natal care in the late 1930s, a healthy diet imposed by rationing in adolescence and early adulthood, being at the right age to benefit given the timing of medical advances in infectious diseases and cardiovascular disease and social changes such as smoking cessation (Dunnell 2008; Goldring et al. 2011; Lessof 1949). Su (2009) suggested that early life factors are becoming less important over time; as infant and child mortality rates are now close to zero in most developed countries, later life conditions would be expected to become more important. Vaupel et al (1998) proposed that one quarter of the variation in adult lifespans could be explained by genetic factors, one quarter by early life conditions and half by conditions in later life. There has been very little further comment on this hypothesis, although van den Berg et al (2011) suggested the formula cannot be generalised: genetic and environmental factors may be more important when economic conditions in early life are poor with individual factors becoming more important for people born in times of better economic conditions. Overlaying all the influences of different factors and the interplay between them, individual mortality prospects are to some extent a matter of chance:

"Individual characteristics are the phenotypic expression of the match or mismatch of our genes with our lifetime environment, coupled with a good dose of chance. ... these individual characteristics, measured in the pre-adult as well as the adult life, are associated with the risk of premature mortality or the chance of surviving to old age."

Kuh et al (2009) p. 50 


\section{International comparisons a promising supplement to mortality analysis}

Given so many open questions, demographic theory on how the multiple potential factors cause population mortality trends, and on the differences in average mortality and its variance between and within populations, is tentative. Biodemography - the idea that biology can inform demographic analysis - has an expanding literature, and has helped to improve understanding of how biomarkers can predict individual mortality risk. However, it is less well understood how trends in population indicators of biological risk might affect future trends in population mortality (Crimmins et al. 2010a; Olshansky et al. 2002; Vaupel et al. 1998).

Another route to understanding trends at the population level appears more promising. International comparisons are highlighted as a rich source of insight in demography (for example Canudas-Romo 2010; Oeppen and Vaupel 2002). This is based on the observations of similarity and difference in the mortality or life expectancy of nations over time. White (2002) shows that among over twenty high income countries the change in life expectancy in individual countries from 1955 to 1996 showed significant convergence toward the group mean. Wilson (2011) demonstrated convergence of life expectancy at birth in the second half of the 20th century between the groups of most developed and developing countries, with exceptions having suffered significant specific mortality setbacks. However, Leon (2011) in remarking on the similarity of trend in life expectancy at birth since 1970 for Western European countries noted how they remained parallel but separate. Thus, analysis of differences between like countries in generally similar mortality environments could inform understanding of the past experience of mortality for any individual country, and therefore add to theories for how mortality 
may change in future. Learning from international comparisons of past mortality trends is urged as best practice for projections of future population mortality for any country (Janssen and Kunst 2007; Li and Lee 2005).

The data available for mortality or longevity analysis between countries is vast. For example, the Human Mortality Database hosted by the University of California, Berkeley and the Max Planck Institute for Demographic Research contains well documented on-line access to population and mortality data for 37 countries or areas, including complete life tables since the 1800s. Summary measures such as life expectancy for areas, regions and countries are also available from organisations including the United Nations and OECD. Demographic literature contains a number of important comparisons of mortality or life expectancy data between countries made in order to investigate specific research questions (including those already referenced). Despite the difficulty in imputing cause to effect with certainty, models of potential future longevity that have been interpreted from observed mortality trends at population level add to demographic theory, even if somewhat qualitative (Burch 2003; Caldwell 1996; Hoem 2008). Thus international comparisons of mortality trends are promising as a source of theoretical and qualitative insight for explaining the past and projecting the future.

\section{Mortality analysis in New Zealand more historic than future}

Past mortality trends in New Zealand have been comprehensively examined (Pool 1982, 1985; Pool and Cheung 2002, 2003). The general trends described above applied in New Zealand. New Zealand had an early reputation as a healthy country for the settler population, with the life expectancy of nonMāori females in New Zealand the best recorded in the world from 1875 to 
1940 (Leslie 1895; Lessof 1949; Oeppen and Vaupel 2002). The advantage the non-Māori population in New Zealand had has been described as "a result of ... a selected, well-fed, well-organized population in combination with a low disease load" (Bengtsson 2006 p. 91). Later analysis decided New Zealand's early first placings were a result of unusual selective healthy migration, and allowed non-Māori females worldwide best placing only for calendar years 1926-1937, 1939-41 and 1944 (Shkolnikov et al. 2011b). Comparing total population average lifespans for generations born throughout the same period shows New Zealand was consistently the best among those countries where such data exists, with a distinct advantage in childhood survival (Statistics New Zealand 2007). Mortality rates in New Zealand have steadily improved since then, but other countries have caught up. Total population period life expectancy at birth in New Zealand in 2007 ranked 13th out of 31 countries in the OECD (OECD 2010).

The state of analysis of current mortality trends in New Zealand is less comprehensive than the understanding of mortality history. Mortality literature in New Zealand either reports on the impact of specific risk factors or medical intervention on specific causes of mortality, or seeks an ethnic or socio-economic cause for mortality differences within the population (Blakely et al. 2007; Blakely et al. 2008; Carter et al. 2010; Tobias et al. 2009). Further, there is little analysis on mortality differentials between New Zealand and other national populations beyond the use of summary life expectancy measures (for example in Statistics New Zealand 2009c, p. 18). The reasons for mortality differentials between New Zealand and other countries are therefore largely unexplored. Although Statistics New Zealand state that "International comparisons of mortality and longevity continue to serve as a useful check on plausibility", they report only that "the New Zealand life expectancy 
assumptions are broadly consistent with the latest available projection assumptions from national statistical agencies in Australia, Canada, Japan, United Kingdom, and the United States" (Statistics New Zealand 2009a). In consequence, there is no integrated analysis of current New Zealand mortality trends at the population level to provide a basis for future mortality forecasting.

\subsection{Mortality forecasting methods}

However much analysis is carried out to explain past and likely future mortality trends, policy makers need assumptions about future population mortality or longevity. These assumptions form the inputs to policy makers' economic models to produce estimates of, for example, the future costs of public superannuation. The estimated future costs inform long-term policy planning. This section reviews the literature on mortality forecasting to explore what demographic theories and methods are used to forecast future mortality.

The assumptions used in policy are developed by national statistics agencies as part of regular forecasts of national population size. The statistical agencies would refer to what are here called forecasts as "projections". The difference between what is meant by forecasts as opposed to projections is significant, and discussed further in the next section, but for convenience, this section continues to refer to forecasts.

National statistical agencies are usually staffed by demographers, but actuaries may also produce national population forecasts related to public pension schemes. Actuarial science may have a limited literature, but it is a 
rich source of knowledge on mortality. Mortality forecasting is at the heart of the theory and practice of actuarial science, because insurance companies and funded pension plans can only be managed by valuations of their future liabilities which depend on when policyholders or pension fund members die. Actuaries first developed the concepts of mortality analysis (Keyfitz 1993; Olshansky and Carnes 1997). Further, demographers have recognised that they have been less comfortable with mortality forecasting than with other aspects of their subject (Crimmins 1993; Preston 1993). The majority of demographic work, as typified by national statistics agencies, is in measuring what has happened. Such agencies usually make forecasts of future national populations only every five years, and forecasting future mortality is only one part of that, with fertility and migration being the other elements. Actuarial literature and practice on mortality forecasting can therefore add to that in demography.

However, there is only a small amount of demographic or actuarial literature specifically assessing approaches to mortality forecasting. Much of this literature comes from the point of view of the technical expert. It reviews variants of forecasting methodology (for example, Booth and Tickle 2008; Booth 2006) or promotes a specific method (for example, Bongaarts 2006; Dowd et al. 2010). Booth and Tickle (2008) describe three methods for making projections of future longevity. The most common is the "extrapolative" method which estimates the values of future mortality rates or other measure such as life expectancy on the assumption that the historic trend of the chosen measure will continue. The "explanation" method uses structural or epidemiological models for separate parts of the mortality rate, which can mean extrapolating mortality rates for individual causes of death. The third method ("expectation") makes forecasts based on expert opinion for outcomes. 
The experts are likely to base their opinions on the future in part on their interpretation of past trends in the mortality rate. Thus, all methods are to a greater or lesser extent dependent on some kind of extrapolation of the past into the future. All methods require some judgement on exactly what past data are extrapolated, and how.

The accuracy of forecasting mortality in industrialised countries improved over the period from the 1950s to the 1980s, in the era of fairly stable mortality rates. From the 1980s, forecasts in European countries became less accurate, with future mortality rates being overestimated, that is, pessimistic about future mortality improvement and underestimating future lifespan prospects (Keilman 1997, 2008). Throughout this period, demographers in different countries were using similar extrapolative methods. In the 1980s mortality rates improved with the surprise of fast improving mortality rates at older ages. As assumptions about future mortality improvement were based on the past, forecasts became less accurate (see also Booth and Tickle 2008 p. 33 and Murphy 1995). At times when the pace of mortality improvement is accelerating, the extrapolative method is likely to underestimate future longevity, as the extrapolative method will always be behind time in identifying improvements. Ever-improving assumptions for mortality change, and ever-lengthening forecast life expectancies, feature in successive national projections.

As a response to criticisms of the simple extrapolation method, more complex variants based largely on stochastic modelling have developed in recent years. Traditional extrapolation models produce one deterministic outcome scenario for each set of input assumptions. Stochastic methods use probabilistic modelling so the output can show a range of possible outcomes 
with associated probabilities. The first stochastic models, notably the LeeCarter method, still did not allow for different rates of change of mortality rates at different ages (Lee and Miller 2001), so further development of stochastic methods has continued, introducing more assumptions and greater complexity in the extrapolative algorithm.

These more complex stochastic models have brought new problems compared to the traditional deterministic methods. Stochastic models are data-intensive, so costly to develop. More assumptions are needed as input and the wider range of possible outcomes appears to increase uncertainty rather than help to explain it. Users generally ignore the added information, preferring to use a single mid-range scenario outcome for simplicity. The model choice may introduce new sources of uncertainty. Although some compromises between fully deterministic and fully probabilistic have been suggested most national agencies still use deterministic extrapolative models (Abel et al. 2010; Booth 2006; Dowd et al. 2010; Long and Hollmann 2004; Lutz and Goldstein 2004; Stoto 1988). However, whether stochastic or not, all such models depend on the time-series of historic data which forms the basis of extrapolation. Regardless of the technicalities of method, longevity forecasts are based on extrapolation.

The use of extrapolative models has been justified on the basis that the improvement in life expectancy over centuries has been remarkably linear, despite being caused by a multiple of social, medical and economic factors (Bengtsson 2006; Oeppen and Vaupel 2002; Tuljapurkar et al. 2000; Wilmoth 2000). However, the extrapolative method at its most simple will turn out to be inaccurate if future patterns in any of the factors - age, gender or other underlying past changes in mortality rates turns out to be different from what 
has happened in the past. Simple extrapolation by merely mechanically following a formula will produce underestimates of future lifespans if actual mortality improvements are hidden within the overall trend or are unexpectedly rapid.

The potential for the future of mortality to be different from its past is a nagging doubt. Some commentators see conservatism in the use of the extrapolative model because it fails to take account of potential disturbances to past mortality trends which are more likely than not to increase lifespans further. De Grey (2006 p. 84) suggests extrapolations are "dangerous" if they are based on "misuse of biology". He suggests highly optimistic forecasts of four-figure future lifespans (which he expects demographers to ridicule) based on "plausible sequences of scientific advances" (de Grey 2006 p. 83). While de Grey urges that an understanding of biology tells us mortality improvement will change beyond recognition, demographers continue to assume the past trend will continue in future, with scope for exercising different judgements about the use of past data (for example, Tuljapurkar 2005). Keilman (2003) calls models that use epidemiological data "non-demographic" and suggests that many demographers lack the experience to work with models of disease causing mortality.

It used to be the case that demographers could defend pure extrapolation on the basis that understanding of biology was not precise enough to translate into projection assumptions (Wilmoth 1998). Given increasing knowledge of and questions about - the drivers of mortality trends, that may change. For example, Olshansky et al (2009) suggested that the US Census Bureau underestimated future population lifespans by assuming a slowdown in the rate of improvement of future mortality when advances in biomedical 
technology could justify assumptions of accelerated improvement. Olshansky is regarded as a pessimistic demographer with specific rebuttals made against his assumptions of the future path of mortality rates (de Grey 2006; Preston 2005; Tuljapurkar 2005).

Also important is new work understanding trends in mortality variance. As the changing nature of mortality variance begins to be understood, demographers are suggesting that pure extrapolation may become less valid in future. In a recent paper examining how variance in lifespans has been changing as mortality compresses, and how variance is mathematically linked to the curve of the rate of aging by age, a former supporter of extrapolative projections now seems less sure that they will continue to be useful:

"Given the long-term nonstationarity of variance, and the rapidity of its decline prior to 1960, it is questionable how helpful extrapolative forecasts may be." Tuljapurkar and Edwards (2011 p. 516)

Therefore, to the scope for judgement in extrapolative forecasting is now added disagreement, if not outright controversy, about its validity in future. Even a technically complex extrapolative model is not immune. An example is Dowd et al (2010) who projected average expected future lifetime for 65 year old males in England and Wales to 2006, using historic data from 1961 to 1980. The projection was carried out using a complex stochastic model, but was essentially extrapolative. The actual population average not only always exceeded the central projection after 1985, but after 2000 was higher than the upper limit of the $90 \%$ prediction interval, and at the very end of the projection period was outside the prediction area. As the authors describe, the forecasts 
are "based on the assumption that the future will be like the recent past...This is a controversial assumption." (Dowd et al. 2010 p. 70). The assumption that extrapolation was valid turned out not to take account of mortality improving faster than past data anticipated. Now, the future might again be different from the past because of mortality shifting and compression. The progress of mortality at the oldest ages has less empirical evidence on which to base theories.

Demographers have been categorised as optimists or pessimists for their judgement on whether compression is up to a fixed limit to average human lifespan and if so, what that limit might be. The classic example of a pessimist is Fries, who in an influential work in demographic literature proposed in 1980 and confirmed with further evidence in 1989 that there would be a limit to human lifespan. Fries envisaged a limit to maximum human lifespan of around 100 years with average lifespan distributed with a mean of 85 years and a standard deviation of 4 years (Fries 1980; Fries 1989). However, Fries's model did not contemplate the impending acceleration of older age mortality improvement. It essentially assumed a linear continuation of overall past trends. Fries is now seen as unduly pessimistic, but the question of whether there will be a limit to human lifespan at all is still a controversial issue for demographers (Carey and Judge 2001; Carnes and Olshansky 2007; Oeppen and Vaupel 2002; Siegel 2005). A consensus appears to be emerging that even if there is a limit then it is not yet relevant, as such a large improvement is needed to bring the average up to the already achieved maximum (Christensen et al. 2009).

This consensus envisages average life expectancy will continue increasing within the period of current population projections. In the UK, five out of the 
panel of six experts who advise on future mortality assumptions for national forecasts said that life expectancy at birth would increase at least as fast over the next 25 years as it has over the last 25 years (Shaw 2008). All 17 experts at a 2007 meeting of European Union (EU) demographers, in a trial of a questionnaire on future trends affecting mortality to develop a systematic approach to defining assumptions for population projections, concluded that the life expectancy measure would continue to increase, although with some uncertainty as to the pace of change (Lutz 2009). The average life expectancy at birth for Japanese females breached Fries's limit in 2000, and the United Nations forecasts that measure to reach 90 years by 2040 and 95 years by 2090 (United Nations 2011). The future population projections from Statistics New Zealand assume a continued improvement of mortality rates. The different variants make different assumptions only for the rate of change (Statistics New Zealand 2009a).

\subsection{Uncertainty in mortality forecasting}

Mortality theories have limited predictive power, and uncertainty in forecasting is inevitable. This section is concerned with how the uncertainty of future mortality and longevity is expressed in the policy sphere.

Academic literature is rather light on the theory and principles behind the use of mortality forecasting in policy settings. There are very few studies of the principles from the demographers' standpoint, Stoto (1988) being a thoughtful exception. Within policy literature, the recent evidence-based policy movement has offered only some general ideas reinforcing that technical information should be sourced from experts, which in this case would be demographers or actuaries (Young et al. 2002). 
Demographers agree that the demographer should be responsible for estimating and presenting the uncertainty inherent in the forecasts presented to policy makers (Stoto 1988). It is more controversial as to how the uncertainty of demographic forecasts should be reported to the user. Keyfitz (1993) highlights the difference between the demographer who prepares a 'projection' of what might happen in the future, given specific assumptions, and a user who wants a prediction of what will happen, and will interpret any demographic projection as such. Keilman (2008) agrees with this assessment of how the user will read a projection, and suggests the term 'forecast' should be used, as that is more descriptive of how a user interprets a development that is possible or likely, but not certain.

Keilman stresses also that users need to understand the uncertainty in any forecast: that there is uncertainty and some indication of how much. He criticises the common practice of statistical agencies presenting deterministic 'high', 'medium' and 'low' variants for population projections with no information on the likelihood of any one of these, or on what needs to happen for the variant to hold. Users tend to pick out the medium variant as the most likely outcome (Goldstein 2004; Keilman 1997).

Empirical evidence on the process of developing official national population projections is available from reports of the national statistical agencies. These reports vary in degree of detail and explanation about how the mortality assumption or assumptions were derived, and on the degree of uncertainty attached to the assumptions. Lutz (2009) surveyed national statistical agencies in the EU in 2005 to review how they prepare their 
assumptions for national population projections, concluding that uncertainty was poorly explained:

"While most users of projections are primarily interested in a best guess forecast, there is increasing demand for explicit consideration of the full range of uncertainty." Lutz (2009) p. 1

However, as discussed in the last section, stochastic forecasting methods, which would provide quantified indicators of uncertainty intervals, are not widely used. Lutz' survey showed that only one national statistic agency in the EU used stochastic forecasting, most produced three variants of deterministic projections, and some agencies produced fewer than three.

In New Zealand, Statistics New Zealand produce national population projections generally within a five-year cycle, based on Census data, with projection period up to 55 years. The methodology used for the latest set of projections is described in Statistics New Zealand (2009a). New Zealand's population projections are presented in nine main series as a high, medium and low variant set of assumptions are used for each of mortality, fertility and migration. This thesis focuses on mortality assumptions only. These are presented in three main series (series 3, 5 and 7), to be referred to here as "Low Mortality", "Medium Mortality" and "High Mortality". Chapter 5 sets out in detail the mortality assumptions and the resulting life expectancy and other measures. The projections for period life expectancy at birth for New Zealand females in 2061 from these variants are, respectively: 91.2 years, 88.7 years, and 86.2 years. An additional series is produced, called here "Very High Life Expectancy" by assuming period life expectancy at birth reaches 95.0 years for both males and females in 2061. 
As expected, because the extrapolative method is slow to catch up with mortality improvements, estimates of future lifespan in New Zealand's population projections have generally been revised upwards over time. Projections of life expectancy at birth have proven to be underestimates. To illustrate from the medium estimate of the 1988-base projections, male life expectancy at birth in 2006 was projected to be 73.9 years. This indicator increased through successive projections, until reaching 77.5 years in the 2004base projections, and the actual outcome was 78.0 years (2005-7 period life tables). Similar patterns are seen for female data and other time periods, with longer projection periods generally increasing the inaccuracy of projections (data obtained from Statistics New Zealand, November 2011).

No explanation is given as to what factors might drive Statistics New Zealand's three main mortality variants, and no probability is assigned to them. The Medium Mortality estimate is set by a traditional demographic technique of extrapolation based on past mortality experience. Projected life expectancy at birth in 2061 for the Medium Mortality estimate is midway between that for the High and Low variants, with no rationale given for the equivalent distance from Medium to the arbitrary High and Low variants. The variants are not suggested as bounds of what is possible or even plausible. Indeed, Statistics New Zealand specifically rejects the Keilman approach stating that its demographic projections are "not predictions or exact forecasts" (Statistics New Zealand 2008 p. 5).

Life expectancy is the summary indicator used to illustrate future lifespans under different mortality projection variants, but Statistics New Zealand downplays its likely accuracy or realism: 
"The mortality assumptions should not be used as a precise measure of mortality or of mortality differentials between groups. It is important to note that the objective of population projections is not to specifically measure or project the life expectancy of the population. For projection purposes it is more important to have a realistic yet tractable model for projecting mortality trends (and death numbers) into the future."

Statistics New Zealand (2009a).

While suggesting that the model is "realistic", the medium estimate is not so defined, although Statistics New Zealand say that it is "considered the most suitable for assessing future population changes" (Statistics New Zealand 2009a). Further, the other projection series "allow users to assess the impact on population size and structure resulting from changes in the assumptions for each of the components of population change" (Statistics New Zealand 2009a). Thus, there is no obvious theoretical underpin for the mortality assumptions other than following an extrapolative method. They are not supported by a comprehensive analysis of the drivers of mortality trends in New Zealand, by information on mortality trends in other countries or by a biological rationale. The user has to decide which if any of the variants are likely or plausible.

Consistent with the Keilman and Keyfitz view, users take the medium estimate of Statistics New Zealand's projections as if it were the most likely prediction. The New Zealand Treasury's long-term fiscal modelling requires forecast data for at least 40 years hence (The Treasury 2006). Uncertainty in this time horizon is significant. Only the medium variant of Statistics New Zealand's population projections is used in the New Zealand Treasury's longterm fiscal statements (The Treasury 2006 p. 41; 2009 p. 18). However, a 
number of other assumptions have been used in other modelling work to illustrate demographic uncertainty (Rodway and Wilson 2006 p.12), and the use in fiscal models of demographic variants as given with no indication of uncertainty has been criticised in a Treasury Working Paper as "seriously flawed" (Bryant 2003 p. 1). Thus despite understanding that uncertainty is poorly treated, the external message in the public Treasury statement is a single deterministic path for mortality: the Medium Mortality estimate.

In the process where expert demographer develops population projections and a non-expert uses them, the complexities of dealing with uncertainty in future mortality are lost. The demographer is unwilling to put probabilistic bounds or rationale on the mortality projections because that would demand a complex, and in most cases unavailable, stochastic model or a biological rationale which is outside the expert domain of the demographer. The solution proposed is usually to develop a stochastic model (Abel et al. 2010; Bryant 2003; Lutz 2009). However, as noted, a stochastic model requires more complex assumptions with probabilities assigned to variables. If these probabilities themselves do not have a rationale, biological or otherwise, then the stochastic model is as open to criticism as a deterministic model. Further, while some policy analysts may be expert in dealing with probabilistic modelling, most other user groups still demand a single forecast, and public messages reference a single forecast that is deemed, rightly or not, to be the most likely. Moreover, even if uncertainties are demonstrated, for example with fan charts as in Dowd et al (2010), the question of how policy decisions would be made differently as a result has hardly been tackled.

Thus more complex stochastic modelling may not be an easy or complete answer. An alternative is to develop the use of deterministic scenarios 
described not by variants of mathematically-based mortality assumptions, but plausible narratives. The use of scenarios to consider what may happen in future as an aid to decision making is established in business management practice where the approach of presenting three alternative scenarios along a single dimension - such as the high, medium and low usually seen in demographic projections - is rejected as it does not challenge the user to think about the future as anything other than a continuation of the present (Wack 1985a, b). Instead, the aim is not to get one scenario right - accuracy is impossible anyway - but rather to illuminate the factors driving possible outcomes of the future by describing a narrative rationale for each alternative scenario. Such a rationale could reflect an observed relationship with other data, such as comparison with trends in other countries, or by a hypothesised future trend based on empirical biological or medical data.

The attraction for a narrative scenario approach in demography is that it meets the criticism that demographic forecasts are purely formulaic. The difficulty is that 'what-if' scenarios can require a better understanding of how risk factors cause mortality outcomes and the trends in those risk factors than exists. For example, a scenario based on 'if the incidence of smoking were to decrease by $X$ per cent then the mortality rate would be likely to reduce by $Y$ per cent' is no more than a sensitivity analysis unless it is based on empirical evidence of cause and effect. Unfortunately, smoking is the single example where empirical evidence of cause and effect exists because the main cause of death from smoking - lung cancer - can be isolated. Other mortality risks have complex outcomes unable to be linked to a change in mortality rate (Crimmins et al. 2010b). As a result, a narrative scenario approach has been little used in demography or actuarial science. While Lutz (1995) proposes what he calls a scenario approach, the term "scenario" is generally used where 'variant' would 
be more appropriate. The actual depth of narrative or rationale supporting assumptions in national population projections is still weak, as Lutz' later survey of EU countries' projections showed (Lutz 2009 p. 4).

Two actuarial examples of long-term scenario-based mortality projections are found in Benjamin (1982) and Calvert (1946). Benjamin presented dramatic new projections for future life expectancy in England and Wales, to general scepticism that his assumed strong mortality improvements could occur (Benjamin 1982 and discussion). However, his forecasts were prescient. The middle of the range of Benjamin's projections for life expectancy in 2017 has already been breached and his "extreme" projection for 2017 is expected to be reached in 2020 (GAD/ONS data). Actual mortality rates in New Zealand in 2000 compared well to those Calvert predicted fifty years earlier in a report commissioned by the New Zealand Government on the future of the country's population at the end of World War II (Calvert 1946 and Statistics New Zealand data). Benjamin developed a rationale based on recent trends and potential new treatments for each cause of death separately. Calvert considered qualitatively the possibility of new discoveries in drug technology, the expansion of "preventative medicine" like the introduction of free milk in schools, and a step change in "improved national vitality" from cutting down hours of work and improving standards of housing (1946 p. 36). Benjamin and Calvert's reports were notable exceptions to the generally inaccurate and pessimistic mortality forecasts of the time, and provide examples of the power of thinking beyond pure extrapolation to reason on the basis of plausible narrative. They are consistent with a model of "less computation, more cogitation" in the making of projections as urged by Hajnal (1955 p. 321). 


\subsection{Conclusions}

The literature reviewed in this chapter suggests that longevity risk is prevalent in New Zealand and elsewhere. Contributory factors to underestimation of lifespans include: over-reliance on historic trends as a guide to the future when mortality is improving; inadequacies in the extrapolative method demographers use to forecast future mortality; and, weaknesses in the communication of demographic evidence to policy analysts that offers a poor explanation of uncertainty in forecasting and little guidance on what evidence for future lifespans to use in policy modelling. To reinforce the hypothesis, studies in countries other than New Zealand referred to briefly here and explored more fully later in this thesis give empirical evidence of a tendency for individuals to underestimate future longevity. The extent of underestimation of lifespans in individual retirement planning and policy settings in New Zealand has not been quantified.

When mortality improvement has been hidden or faster than expected then the use of extrapolative forecasting techniques tends to underestimate longevity. Successive projections of future lifespans have consistently had to be revised upwards. However, while demographers forecast mortality, some reliance on extrapolation seems inevitable. In the judgement required for extrapolation there still lies scope for relative pessimism or optimism and major disagreements over the course of future mortality trends. While the weight of demographic opinion is that mortality will continue to improve, the pace of future improvement is not agreed. Questions over the future validity of pure extrapolative forecasting add further to the controversy of the technique. The consequences of compression of mortality and the implications of changing mortality variance on population average mortality or life 
expectancy are not fully understood. Extrapolative methods will be slow to react to change in the pace of mortality rate change. Thus the risk of underestimation of future lifespans still exists, in an overall context of uncertainty. All of these points apply to developed countries where mortality had been generally improving over recent decades, including New Zealand.

Alternatives to mitigate the problems of mortality forecasting have been suggested, but have not been taken up widely. Ever more complex forecasting models do not seem to be the answer when there is insufficient understanding of the factors driving mortality rate change. Instead, narrative scenarios of future longevity appear a promising tool consistent with Hajnal's view that projections would benefit from less computation or pure extrapolation and more descriptive consideration of potential major trends. International comparisons of mortality trends have been suggested as a promising source of theoretical and qualitative insight for projecting future mortality. There has not been a thorough comparison of New Zealand's past or future projected mortality trends with those of other relevant countries.

Despite indications that longevity risk exists, in New Zealand and more generally, and some analysis within annuity or insurance settings, the extent of longevity risk in policy and by individuals remains unexplored. The reasons for any underestimation of longevity have not been addressed or remedies suggested. Thus while evidence accumulates to suggest an inherent tendency to underestimate future longevity in both policy and individual settings, existing literature leaves a research gap on the significance of longevity risk. 
Underestimating lifespans? Why longevity risk exists Chapter 2

This page has been intentionally left blank. 


\section{Chapter 3: Theoretical basis for mortality analysis}

This thesis undertakes mortality analysis for New Zealand in order to provide plausible future lifespans against which to test the expectations of individuals and policy makers. The theoretical basis for the mortality analysis in this thesis is set out in this chapter. First, the theory behind the most common measure of longevity - population average life expectancy - is explored in depth. The second section describes the three-part framework developed to carry out the mortality analysis in this thesis and the theory behind it. The following three sections give the details of the source data used to carry out the analysis. The results of the analysis then follow in the subsequent three chapters, with additional theory on the third part of the framework in the relevant chapter.

\subsection{Flaws in life expectancy measure}

Longevity and mortality are complex technical subjects, yet of great interest to non-specialists. Summary results from detailed analyses are often presented using a simplified interpretation with few measures. The most commonly used measure of longevity is life expectancy. Life expectancy at any age is the average remaining lifespan for the population the mortality data represents (Preston et al. 2001). Life expectancy at birth is the average total lifespan, or equivalently average age at death, for the defined population. Life expectancy is a useful measure for some purposes but it suffers from significant definitional and technical drawbacks as a means of conveying information on likely lifespans for individual decision making or national policy making, as the remainder of this section considers. 


\subsubsection{Period life expectancy underestimates real lifespans}

For any population, average life expectancy at any age greater than zero plus that age is greater than life expectancy at birth, because of selective survival. Even if mortality rates did not change over time, older people would have higher chances of longer lifespans than younger people. Thus life expectancy at birth only applies as a measure of total lifespan to newborns represented by the underlying population. Period life expectancy at birth is a poor indicator of potential lifespan for any ages over zero, or to represent the average for a population with a range of ages.

Life expectancy, as conventionally used, will not indicate a realistic lifespan. Usually in demographic sources "life expectancy" denotes a period life expectancy indicator, which is an artificial statistic. Period life expectancy is calculated assuming mortality rates at each age that apply in a single period (usually a calendar year) for a population. It is the average age at death for a population at that point in time assuming no change in mortality rates over the lifetime of the people in that population. But in reality, mortality rates change over time. A period life expectancy is therefore clearly artificial when applied to an individual person. An individual experiences the mortality rate at each subsequent age that applies in subsequent years. A more realistic and intuitive indicator of average lifespan, which reflects the changing of mortality rates with age, and therefore time, is cohort life expectancy, calculated using the average mortality rates at each age of a population of people born in the same year. 
Period and cohort life expectancies at birth $\left(\mathrm{e}_{0}\right)$ for the period $\mathrm{t}$ and the cohort born at time $t$ respectively are functions of death rates for ages at different times:

$$
\begin{gathered}
\text { Period } e_{0, t}=f\left(q_{0, t}, q_{1, t}, q_{2, t}, \ldots\right) \\
\text { Cohort } e_{0, t}=f\left(q_{0, t}, q_{1, t+1}, q_{2, t+2}, \ldots\right)
\end{gathered}
$$

Period life expectancy cannot represent a real expectation for any person. Only cohort life expectancy indicators take into account the lifecourse dimension of mortality change. When age-specific mortality rates improve over time, as is currently the case in all developed countries, a period life expectancy indicator will underestimate a realistic average lifespan. Period life expectancy for age $\mathrm{x}$ at time $\mathrm{t}$ will be lower than the cohort life expectancy for a cohort aged $\mathrm{x}$ at time $\mathrm{t}$. Cohort life expectancy will give a more realistic indication of a lifespan as it applies to an individual from the relevant population. The realism of cohort life expectancy as an indicator of lifespans for cohorts still alive will be subject to the plausibility of the assumptions made about future mortality, but the realism of period life expectancy is limited because of the implicit assumption that mortality rates do not change.

The need for assumptions of future mortality rates when calculating cohort life expectancy for a cohort still alive need not pose a problem where assumptions already exist, usually for population projections. However, it requires more calculations from the usual requirements of the more commonly produced period life tables, and cohort life expectancies are less often referenced than period indicators. 
The difference between period and cohort measures is understood within demographic and actuarial literature, as is the hypothetical nature of period life expectancy as a measure of lifespan (for example, Vaupel 2001). However, demographic literature has focused on exploring the so-called "tempo effect", a theoretical concept used in measuring fertility rates, as an explanation for the difference between period and cohort life expectancy. Studies on alternative measures for average lifespan, whether from the point of view of population dynamics (Wilmoth 2005) or to capture the true lifespan potential of an individual (Ediev 2011) have explored theoretical mathematical solutions based on the tempo effect, despite cohort life expectancy measures being a practical option. Further, the implications of the differences between period life expectancy and cohort life expectancy in the current era of improving mortality do not have a wide literature in demography. For example, having been first observed by Wilmoth (2005) that period life expectancy will increase more slowly than cohort life expectancy, Shkolnikov et al only recently (2011b) showed that the trend line for the highest national female cohort life expectancy at birth in the world for cohorts born 1870-1920 had a markedly steeper slope than that for best period life expectancy. Not only does cohort life expectancy reveal higher lifespans than apparent from period life expectancy data, but also faster increasing lifespans. Thus, the conclusions of much of theoretical and empirical demographic analysis based on period life expectancy may not apply to the progress of real lifespans from an individual lifecourse perspective which can, however, be analysed using cohort life expectancy. 


\subsubsection{Misrepresented as individual lifespan expectation}

Period life expectancy is a useful indicator for summary comparisons of population mortality across countries at a point in time or across time periods. For example, in the standard source for national population estimates and projections, the United Nations' "World Population Prospects", the selected mortality indicators used to compare across countries are the crude death rate, the infant mortality rate and period life expectancy at birth (United Nations 2011). Period life expectancy at birth is one of the components of the United Nations' Human Development Index, and epidemiologists use life expectancy as a summary measure of the health of a population, as will be discussed in Chapter 6. Thus, period life expectancy is widely referenced, more so than cohort life expectancies, and the most common indicator is period life expectancy at birth.

It is recognised that policy makers tend to use and communicate period data in preference to cohort data (for example, Booth and Tickle 2004), but the difference between the two is rarely explained in informal settings. A casual reader can easily interpret period data as if it applied to his or her cohort. In practice, life expectancy, particularly period life expectancy at birth, is often incorrectly reported as if it should be an individual's expectation, in the sense of an entitlement to a remaining length of life, rather than an average measure for a hypothetical population. For example, this common claim for the state pension age would be true only if mortality rates each year in the life of "the average man" (lifetime undefined) were as they were in a specific year: 1948. 
"When Beveridge brought in the state pension, kicking in for men at the age of 65 , the male life expectancy was 64 . In other words, the average man died before he could claim any state pension at all."

Lanchester (2010) in the Guardian Weekend 2 October 2010

Formal research is not immune to the problem. For example, a significant and detailed study from a large team of demographers and epidemiologists contained the following interpretation from period data:

"In 2006, life expectancy in the United States at age 50 was 32.6 for white women and 30.2 for black women; white men at age 50 could expect to live 29.0 years on average and black men 25.2 years."

Crimmins et al. 2010b p. 9-5, emphasis added

Table 3.1 gives examples of such misreporting of life expectancy in media and policy settings in New Zealand. In the first example of Table 3.1, 82.2 years is the period life expectancy at birth assuming the mortality rates at each age that prevailed for New Zealand females in the period 2005-7 (Statistics New Zealand Total Population Period Life Tables). However, the cohort life expectancy at birth for the average female New Zealander born in 2006 ranges from 86.7 to 93.8 years depending on the assumptions made on the pace of mortality improvement in future (Total Population Cohort Life Tables consistent with 2009-base projection assumptions, obtained from Statistics New Zealand November 2009). Thus, period life expectancy used without explanation leads in this case to a potential underestimate of what can be expected as average lifespan of between 4.5 and 11.6 years. 
Table 3.1: Examples of confusion in use of life expectancy measure

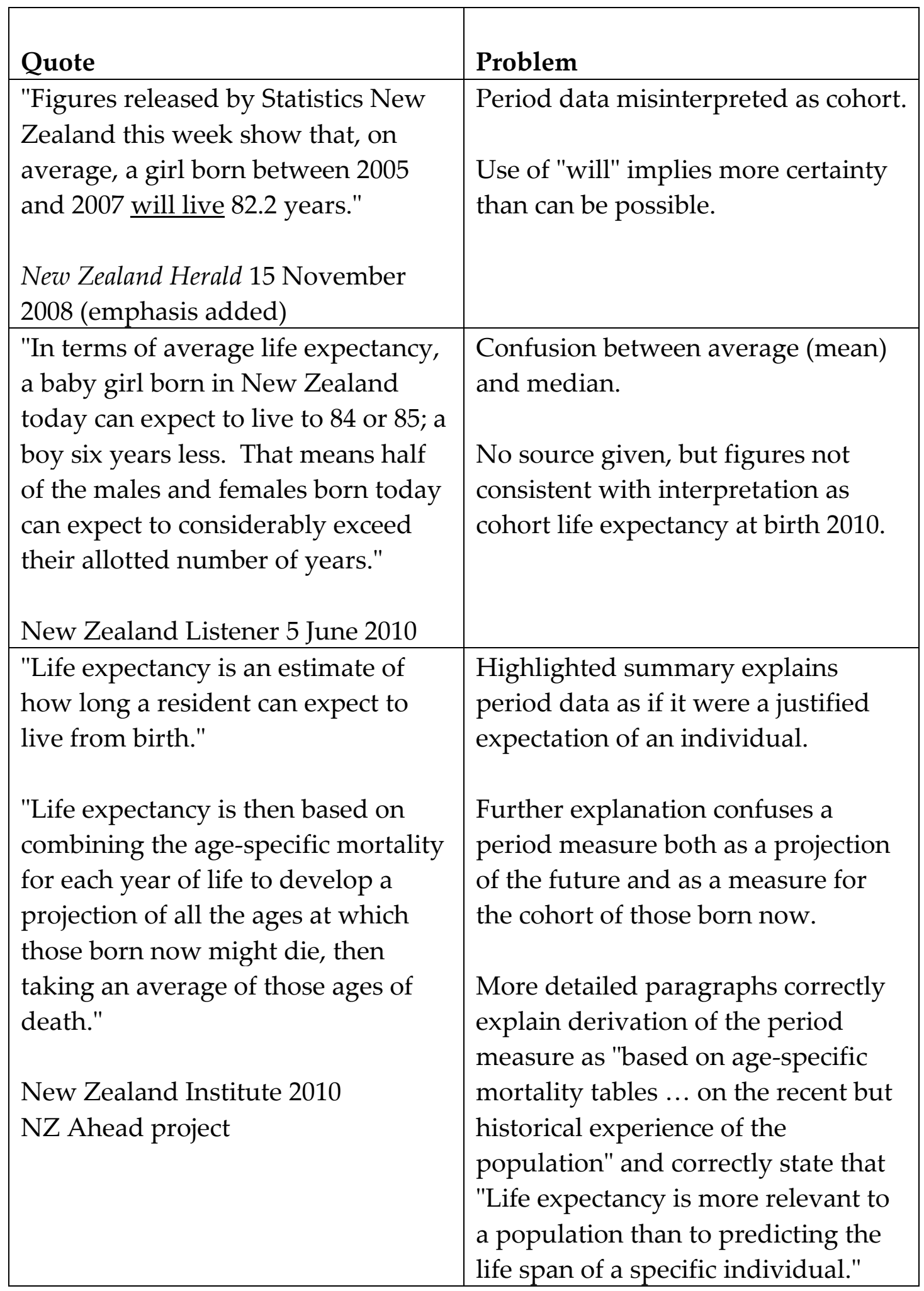


Much of the confusion appears to stem from a casual interpretation of life expectancy as the lifespan someone should expect. It is possible that the confusion between individual expectation and population average was set when the life expectancy measure was first defined. The Oxford English Dictionary (OED) traces life expectancy back to the first recorded use of the term "expectation of life" in one of the earliest works on annuities by the mathematician de Moivre:

"The expectation of life is that duration which may be justly expected from a life of a given age." de Moivre (1725) p. iv, as cited in OED (second edition 1989, accessed online 28 July 2010)

However, the OED extracted only part of de Moivre's definition. The full paragraph in the original text is as follows:

"The expectation of life is that duration which may be justly expected from a life of a given age; and is properly a medium between the longer and shorter durations of a great number of preceding lives, from the time of their having attained the age given, to the time of their extinction. With the present value of this expectation, the proprietor of a life may be said, in some sense, to have purchased an annuity for life, of which the rent is paid him in actual duration, and thereby to have taken his chance of an uncertain duration, as an equivalent for the fixt duration he is entitled to by the right of his expectation." de Moivre (1725) p. iv (emphasis added) 
The full definition makes it clear that expectation of life was the result of an averaging calculation for a defined population (the "medium" of a "great number of preceding lives") and de Moivre sets out the calculation in later pages (pp. 74-80). Further, the definition refers to expectations in the sense of a real entitlement not to a length of life, but to a financial contract; an annuity. At the time, "expectation" was commonly used as a legal term for the prospect of inheriting money. The primary purpose of de Moivre's paper was to set out for the first time the formulae to calculate the cost of annuity purchase. The definition of expectation of life was a secondary product of the mathematics required for the annuity calculation. Thus 'life expectancy' descends from a term intended to convey a specified population average but confusingly defined in the context of an individual's entitlement to a payment for the rest of life. Modern usage of life expectancy is not often in the context of a financial contract, but in the sense of potential future lifespan for an individual or the average for a population. The uncertainties around the mean inherent in a population average measure are often lost, and 'life expectancy' is often wrongly interpreted to mean what lifespan an individual can or should expect. If the other phrase de Moivre coined for remaining lifespan "the Complement of Life" (de Moivre 1725 p. 75) had been adopted instead, some confusion may have been avoided.

\subsubsection{Inadequate measure as mortality compresses}

Life expectancy at birth is the average value along the curve of deaths. The average value along a curve is most useful when the curve is symmetric. However, over time, the shape of the curve of deaths has changed significantly and is not symmetric. As explained in section 2.2, the changing shape of the curve of deaths and the rectangularisation of the survival curve as mortality 
shifts and compresses has occurred for all developed countries, as was shown for the UK on page 26. The curve of deaths for New Zealand females is shown in Figure 3.1. Data for the total population is not available for the full time period, but an earlier line added for the Non-Māori population in 1934-38 illustrates the higher childhood and newborn mortality at that time.

Figure 3.1: Curve of deaths from 100,000 births for females in New Zealand from three period tables, with mean, median and mode

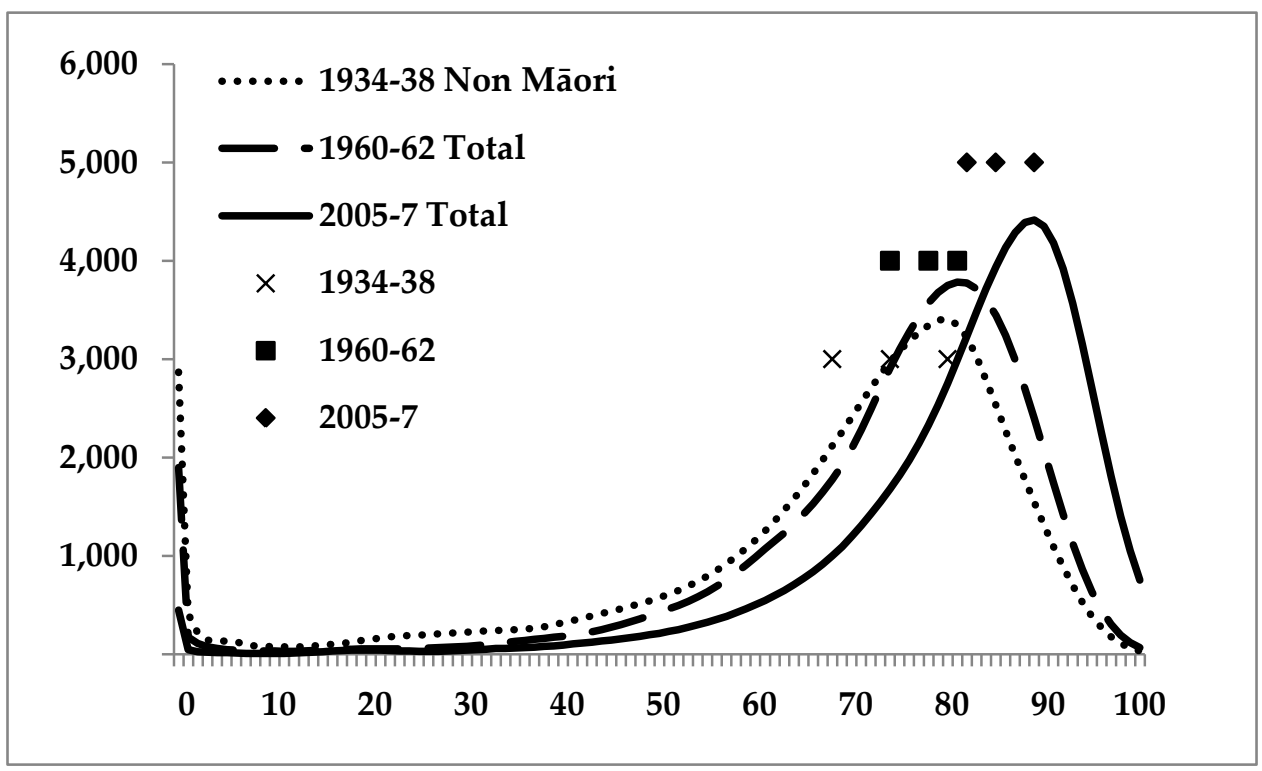

Source: Calculated from Statistics New Zealand period life tables. Note life table for 1934-38 is for the Non-Māori population only. Total population life tables were not produced for that period. For the two later periods, total population data is shown. Period measures are used as cohort life tables are not available for birth years since late 1930s. The three symbols for each time period show, reading left to right, mean, median and mode.

In recent decades, compression of mortality has shifted the second modal age at death to the right, and the peak has narrowed as more people have survived to older ages but maximum lifespan has not increased so significantly. Over the period shown in Figure 3.1, the curve of deaths has changed from being clearly bimodal to a practically single mode distribution 
concentrated at the higher values. The curve of deaths is less symmetric than it was when the peak for newborn deaths was higher, and the use of the average - that is, life expectancy at birth - is therefore questioned (CanudasRomo 2010).

Gains in life expectancy at birth have come from improvements in mortality rates at different ages: in the first half of the 20th century from young ages and in the second half of the 20th century mortality improvements were concentrated at older ages. Life expectancy, especially period life expectancy, does not distinguish well between the age at which mortality is improving most, and the way in which it changes to mortality rate change varies as the shape of the curve of deaths changes. Even if large improvements in mortality rates at older ages occur in a population that is well advanced on the path to mortality compression, life expectancy at birth will not change as much as it did when there was large improvement in mortality at younger ages. An alternative expression of this is that the increase in life expectancy at birth from the same rate of improvement in age-specific death rates would be expected to be smaller in a population further down the pathway to mortality compression than another. The compression of mortality acts as a brake to gains in life expectancy at birth.

Which mortality change at older ages - mortality shifting or mortality compression - currently dominates in developed countries or will dominate in future is debated. Recent data for Canada, France, Japan, and the US suggests that the female peak age at death has increased and variability above the peak reduced, that is, mortality shifting has been most in evidence (Ouellette and Bourbeau 2011). Life expectancy, especially life expectancy at birth, does not give full information to explain these mortality trends. Further, the period life 
expectancy indicator has been criticised for misleading analysis of these trends because by ignoring the potential for future mortality improvement it tends towards showing mortality compression rather than shifting (Ediev 2011). Thus, the usefulness of life expectancy as an indicator of mortality trends reduces for developed countries well advanced on the path towards mortality compression (recognising that this description itself may turn out to be inaccurate if mortality shifting is also a significant element). This conclusion supports the assertion of Olshansky et al that:

"...life expectancy at birth is not an adequate metric of mortality declines when life expectancy at birth approaches 80 years" Olshansky et al 1990 p. 638.

If life expectancy should not be the only measure used for investigating trends in mortality, other measures of central tendency of the population age at death can be considered. In actuarial science, the distribution of deaths around a modal age is considered an insightful measure of mortality trends (Benjamin 1982). Demographers have suggested that trends in life expectancy at birth, the median and adult modal ages at death together provide a basis for better explanation of mortality trends in developed countries than any one of those measures alone (Canudas-Romo 2010; Cheung and Robine 2007). This is because each of these measures of central tendency changes differently to mortality changes above or below the measure in an era when mortality rates are generally reducing (Table 3.2). 
Table 3.2: Effects on measures of longevity of changes in mortality when mortality is generally improving

\begin{tabular}{|lcc|}
\hline $\begin{array}{l}\text { Measure } \\
\text { Life expectancy } \\
\text { (average age at death) }\end{array}$ & $\begin{array}{c}\text { Changes at ages } \\
\text { below the measure }\end{array}$ & $\begin{array}{c}\text { Changes at ages } \\
\text { above the measure }\end{array}$ \\
Median age at death & Yes & Yes \\
Modal age at death & Yes & No \\
\hline
\end{tabular}

Source: Based on Canudas-Romo 2010 Table 1. "Changes" mean reductions in age-specific mortality rates.

Measures of central tendency for the age at death distribution are also marked on Figure 3.1. These measures are in the same order for each period: mean (that is, life expectancy at birth), median and mode. The figures are shown in Table 3.3. The measures exhibit the pattern consistent with that in all developed countries as a result of the age profile of mortality rate changes over the period (Canudas-Romo 2010): the three measures converge over time, staying in the same order, and the increase in the modal age accelerates in the later years. This can be seen in the New Zealand data as an extension to the right of the range of the three points. The shift to the right of modal age at death reflects mortality rate improvements at the oldest ages.

Table 3.3: Measures of central tendency for age at death, New Zealand females

\begin{tabular}{|l|c|c|c|}
\hline & $\begin{array}{c}\text { 1934-38 } \\
\text { Non Māori }\end{array}$ & $\begin{array}{c}\mathbf{1 9 6 0 - 6 2} \\
\text { Total }\end{array}$ & $\begin{array}{c}\mathbf{2 0 0 5 - 7} \\
\text { Total }\end{array}$ \\
\hline Average (e) & 68.5 & 73.8 & 82.2 \\
Median & 74.3 & 77.9 & 85.4 \\
Mode & 80.2 & 81.8 & 89.3 \\
\hline
\end{tabular}

Source: Calculated from Statistics New Zealand period life tables. 
In common with other developed countries, New Zealand has had recent mortality improvements at the oldest ages, life expectancy at birth exceeds the benchmark of 80 years, and modal age at death is extending to near 90 years. The mode changes when mortality rates change at the oldest ages, which is the age range of interest in ageing populations. Modal age at death is rarely used yet could go some way to mitigate the underestimation of lifespan that use of period life expectancy at birth produces. While cohort life expectancy is the intuitively and technically preferred measure of individual lifespans, modal age at death, even from a period life table, provides a simple and easily available indicator of population lifespan of a similar order to the cohort measure.

\subsection{Framework for mortality analysis}

As highlighted in the literature review of Chapter 2, international comparisons are urged as a rich source of insight in demography and specifically for projections of future mortality. This study builds on the premise that comparisons of New Zealand's mortality with that of other relevant countries will add valuable insights to projections of future mortality in New Zealand; and thus yield a deeper understanding of future lifespans in New Zealand than exists in official projections.

Best practice mortality projections have been described by Janssen and Kunst (2007, referring to Li and Lee 2005). One recommendation is to compare data on past mortality trends across like countries and for the two genders within a country to gain insights to use in future mortality projections. The process suggested is partly qualitative - do the relativities in past and future trends across compared populations look reasonable? - and part quantitative: 
mortality for males and females can be extrapolated separately using factors which constrain the difference between average mortality for those two groups to be constant. In this study, I have rejected imposing any constraint on mortality levels in favour of using the qualitative insights gained from comparative analysis of past mortality trends between countries and between subgroups of New Zealand population. Specifically, given the importance of the Māori and migrant populations within New Zealand, the mortality of subgroups defined by ethnicity and birthplace is considered. Additionally, emerging demographic theories on variance of mortality are explored, following the suggestion from Tuljapurkar and Edwards (2011) that change in variance will have an impact on extrapolation. A qualitative approach is necessary as the demographic theories linking variance in age at death with changes in mortality rates and life expectancy may be able to add insight, but are not yet fully formed to be definitive.

Other recommendations for best practice in mortality extrapolation are to explore specific causes of death, and to review whether the mortality trends of younger cohorts differ from that of older cohorts. In this study, mortality rates are considered by age, so that mortality of younger cohorts can be considered directly. Mortality risk or cause of death trends are not considered further, because of limitations in relating cause and effect. However, the potential consequences of a commonly cited mortality risk - obesity - are covered qualitatively.

The dependence on qualitative insights from comparative analysis of past trends in average population mortality adds some judgement to the mostly mathematical approach of extrapolation. However, qualitative theorising is not new in demography. It allows exploration of a range of plausible 
outcomes. Models of potential future longevity postulated from observed mortality trends at population level add to demographic theory, even if somewhat qualitative (Burch 2003; Caldwell 1996; Hoem 2008). The philosophy I adopted for this study is that of 'less computation, more cogitation' in the making of projections, as urged by Hajnal (1955).

The new framework developed in this thesis for this study has three main analytic building blocks:

- Comparing past mortality between countries: Identifying the notable features of New Zealand's mortality trends relative to those of similar countries.

- Comparing future mortality projections between countries: Taking the findings from the between-country mortality trend analyses to test the consistency and plausibility of New Zealand's mortality projections.

- Assessing within-country trends: Learning from emerging theories of mortality variance and assessing available empirical evidence to test whether mortality trends within subgroups of the New Zealand population are likely to disturb the path of projected future total population lifespans. Further detail on the theory behind this part of the framework is in Chapter 6.

The question is not whether each country has followed a suitable method of projection. Rather, given the relative performance of past mortality trends do the relative projected future mortality trends appear reasonable? Are there notable trends within New Zealand's mortality which could cause future mortality to behave differently relative to the group of countries, or which could disturb the extrapolation of New Zealand's total population mortality? What does this imply for New Zealand's mortality projections: do they appear 
relatively optimistic or pessimistic; what is a plausible benchmark for future population lifespans in New Zealand?

For the purposes of comparing mortality trends between countries, long time periods can be used where consistent data exist. Seminal theories in demography have used over 100 years of data. For example, Oeppen and Vaupel (2002) demonstrated world-record life expectancy from 1840. A comparison between the populations of New Zealand and England and Wales of life expectancies at various ages was able to be made for birth cohorts from 1876 because both countries have developed cohort life tables from historic mortality data (O'Connell and Dunstan 2009). However, more recent data has been the basis for other relevant theories. For example, Tuljapurkar et al (2000) confirmed there has been a constant rate of mortality improvement among G7 countries since 1950. Analysis of mortality by birth cohort has been carried out using data since the 1950s or 1960s (Andreev and Vaupel 2005; Gallop 2008).

The purpose of international analysis in this thesis is not to investigate a widely-applicable demographic theory on a world-wide basis, but rather to isolate trends and theories relevant to one country. This perspective is less well documented in demographic literature. A recent example is a study for the US National Research Council which used twenty-five years of data from 1980 to investigate causes for the differences between longevity in the US and other high-income countries (Crimmins et al. 2010b). Given the change in pace of improvement in older age mortality since the 1990s, recent data on mortality trends is likely to be more relevant for the purposes of developing projection scenarios than historical data. 
For this study, the recent period during which mortality compression becomes a feature is most relevant. Therefore, the decision was made to use data from 1961 to 2006. This time period fits with the production of New Zealand total population period life tables which are published every five years. For this study, the analysis period starts with the 1960-62 table and ends with the most recently published at the time of writing which covers the period 2005-7. Prior to the 1950-52 tables, life tables were produced only for the non-Māori population. A consistent time series therefore needed to start after that date, and a decision was made to start in 1960-62, allowing insight into the decades 1960s, 1970s, 1980s, 1990s, 2000s and 1996-2006. This study therefore provides a modern narrative of mortality in New Zealand and its comparison with the selected countries. A longer term history of the tables and trends is in Statistics New Zealand (2009c).

\subsection{Selection of comparator countries}

The choice of comparative countries is made to keep the analysis relevant and focused. The Preston curve (Bloom and Canning 2007; Preston 1975) demonstrates that life expectancy varies little between the world's richest countries, including New Zealand. This establishes that a relevant comparison of New Zealand's mortality can be made restricting the comparison to developed countries. Countries of similar economic development have had similar mortality trends on average in the recent past, although the differences can be illuminating (Leon 2011; Tuljapurkar et al. 2000; White 2002). Janssen and Kunst (2007 p. 323) recommend using the mortality experience of countries with similar socio-economic, cultural and medical technology developments to "create a broader empirical basis for the identification of the most likely long-term trend". 
New Zealand, by population count, is majority European in ancestry, and within that group predominantly British. The United Kingdom (UK) was the dominant source country for migration to New Zealand for one hundred and fifty years, up to the 1950s (Phillips and Hearn 2008). From the 1960s, emigration to New Zealand has become more ethnically diverse, with significant inflows from Pacific Island nations, primarily Polynesia. Migration from Asian countries, always present, increased from the 1980s and recent migration has come from the Middle East, Latin America and Africa. People continue to settle in New Zealand from countries with large populations of European descent, and migration from the UK continues. Australia, Canada and the United States (US) are also British settler countries which have had to some extent similar social histories. For example, public health initiatives and health services that can be assumed to affect mortality have developed similarly, although the US health system in its lack of universal health care is the most different. Therefore, the UK and the British settler countries Australia, Canada and the US offer themselves as suitable mortality comparators to New Zealand.

All these countries publish detailed population and mortality data. In New Zealand mortality projections are produced solely by a national statistics agency working within the demography discipline. In the four other countries mortality projections are produced either separately by an actuarial office within a government department or jointly between the two offices (for further detail, see section 5.1). Thus, these selected comparator countries offer new insights available from supplementing existing demographic methods with an actuarial approach. 
The following data tables offer evidence that this group of five comparator countries has a sufficiently interesting range of difference on demographic and economic measures, even though there is a similar British dominance in population mix. Table 3.4 shows population data. Net migration rates will be volatile and hide flow sizes, but on the basis of the partly-projected figures estimated here, all countries have higher net migration than New Zealand. The proportion of overseas-born residents in Australia, Canada and New Zealand is roughly double that of the UK and US. In each settler country people born in China constitute the first, second or third largest overseas-born group and is likely to be rising in each case as Chinese migration continues. The importance of the number of migrants from the UK is confirmed as UKborn people comprise the first or second largest overseas-born group in each British settler country except the US. Birth country gives some comparable information on population mix, but does not necessarily signal ethnicity. Ethnic mix is more difficult to compare as the purpose and definition of measures of ethnicity vary between countries. While a full analysis of the ethnic differences between each of the British settler countries examined here is out of scope for this study, some data points are offered to illustrate in Table 3.5. 
Table 3.4: Population statistics for selected comparator countries

\begin{tabular}{|c|c|c|c|c|}
\hline & $\begin{array}{l}\text { Total population, } \\
\text { millions } \\
\text { (a) Latest mid-year } \\
\text { (b) in year of birth } \\
\text { country data if } \\
\text { earlier }\end{array}$ & $\begin{array}{l}\text { Popu- } \\
\text { lation } \\
\text { born } \\
\text { overseas, } \\
\text { per cent } \\
\text { of total }\end{array}$ & $\begin{array}{l}\text { Largest birth } \\
\text { country groups } \\
\text { (per cent of all } \\
\text { overseas-born) }\end{array}$ & $\begin{array}{l}\text { Net } \\
\text { migration } \\
\text { rate per } \\
1,000 \\
\text { population } \\
2005-10\end{array}$ \\
\hline Australia & $\begin{array}{l}\text { (a) } 22.3 \mathrm{~m}(2010) \\
\text { (b) } 21.4 \mathrm{~m}\end{array}$ & $26 \%$ & $\begin{array}{l}\text { UK }(21 \%) \\
\text { New Zealand } \\
(9 \%) \\
\text { China }(7 \%)\end{array}$ & 4.8 \\
\hline Canada & $\begin{array}{l}\text { (a) } 34.1 \mathrm{~m} \mathrm{(2010)} \\
\text { (b) } 31.2 \mathrm{~m}\end{array}$ & $20 \%$ & $\begin{array}{l}\text { China }(11 \%) \\
\text { UK }(9 \%) \\
\text { India }(7 \%)\end{array}$ & 6.3 \\
\hline $\begin{array}{l}\text { New } \\
\text { Zealand }\end{array}$ & $\begin{array}{l}\text { (a) } 4.37 \mathrm{~m} \mathrm{(2010)} \\
\text { (b) } 4.0 \mathrm{~m}\end{array}$ & $22 \%$ & $\begin{array}{l}\text { UK }(28 \%) \\
\text { China }(10 \%) \\
\text { Australia }(7 \%)\end{array}$ & 2.4 \\
\hline $\begin{array}{l}\text { United } \\
\text { Kingdom } \\
\text { (UK) }\end{array}$ & $\begin{array}{l}61.8 \mathrm{~m} \text { (2009). } \\
54.8 \text { million in } \\
\text { England and } \\
\text { Wales. }\end{array}$ & $11 \%$ & $\begin{array}{l}\text { India }(10 \%) \\
\text { Poland }(7 \%) \\
\text { Pakistan }(6 \%)\end{array}$ & 3.1 \\
\hline $\begin{array}{l}\text { United } \\
\text { States of } \\
\text { America } \\
\text { (US) }\end{array}$ & 307m (2009) & $13 \%$ & $\begin{array}{l}\text { Mexico }(30 \%) \\
\text { China }(5 \%) \\
\text { Philippines }(5 \%)\end{array}$ & 3.3 \\
\hline
\end{tabular}

Sources and notes: Compiled from data downloaded from official statistical agency websites 17 February 2010. Net migration rate data from United Nations (2011) estimates (Medium variant). China includes Taiwan and Hong Kong except where data unavailable due to small size.

Australia: ABS (2010), birth country data for 2008.

New Zealand: Statistics New Zealand Tourism and migration 2007 statistics, data from Census 2006, as per cent of census usually resident count population shown at (b). (a) is estimated resident population.

Canada: Statistics Canada, data from 2006 Census.

UK: Birth country data is 2009/10 estimated from Annual Population Survey (APS) and Labour Force Survey (LFS), ONS.

US: Census Bureau 2009 American Community Survey (Brief issued October 2010). 
Table 3.5: Summary of available statistics which illustrate ethnic mix for selected countries

\begin{tabular}{|l|l|l|}
\hline & Year & Data and brief explanation \\
\hline Australia & 2006 & $\begin{array}{l}\text { Resident indigenous population estimated at } \\
\text { 2.5 per cent of the total Australian population. }\end{array}$ \\
\hline Canada & 2006 & $\begin{array}{l}\text { Nearly 100 per cent of residents identify with } \\
\text { the Canadian ethnic group or one denoting a } \\
\text { European country, estimating from Census } \\
\text { data similarly to the New Zealand method } \\
\text { with multiple responses allowed. } \\
\text { "Visible minority population" estimated at 16 } \\
\text { per cent of total population. }\end{array}$ \\
\hline $\begin{array}{l}\text { New } \\
\text { Zealand* }\end{array}$ & 2006 & $\begin{array}{l}\text { 80 per cent of the usually resident New } \\
\text { Zealand population identified with the } \\
\text { "European" ethnic group (including New } \\
\text { Zealander and individual European countries, } \\
\text { mainly British), 15 per cent with Māori, 9 per } \\
\text { cent Asian and 7 per cent Pacific peoples. Note } \\
\text { that the term "European" has a common use in } \\
\text { New Zealand not found in other countries. } \\
\text { (Us)* }\end{array}$ \\
\hline $\begin{array}{l}\text { See also Table 6.3. } \\
\text { (UK) }\end{array}$ & 2010 & $\begin{array}{l}\text { 92 per cent of the UK population was in the } \\
\text { "White" ethnic group and 8 per cent in the } \\
\text { heterogeneous "Minority Ethnic" group. }\end{array}$ \\
\hline $\begin{array}{l}\text { Demographic profiling is by "race" and } \\
\text { "Hispanic or Latino". 16 per cent are in the } \\
\text { latter group. } \\
\text { By "race alone or in combination with one or } \\
\text { more other races": 75 per cent are "White", 14 } \\
\text { per cent "Black or African American", 6 per } \\
\text { cent Asian. }\end{array}$ \\
\hline
\end{tabular}

Sources: Compiled from data downloaded from official statistical agency websites 17 February 2010: ABS, Statistics Canada, Statistics New Zealand, ONS, US Census Bureau (downloaded 1 September 2010).

* Figures do not add to 100 per cent because multiple responses are allowed (for race in the case of the US). Figures for smaller categories not given. 
Table 3.6 shows economic and summary life expectancy data. Again, the US is seen as an outlier in the size of its GDP and the highest level of income inequality and the worst life expectancy of this selected group. New Zealand has the lowest GDP and GDP per capita of the group. It is in the middle of life expectancy ranking, with Australia providing a relevant high life expectancy country for comparison.

Table 3.6: Economic and life expectancy indicators for selected countries

\begin{tabular}{|l|c|c|c|c|c|}
\hline & GDP & GDP & $\begin{array}{c}\text { Gini } \\
\text { index for } \\
\text { income }\end{array}$ & \multicolumn{2}{|c|}{$\begin{array}{c}\text { OECD life } \\
\text { expectancy } \\
\text { ranking }\end{array}$} \\
\cline { 5 - 7 } & $\begin{array}{c}\mathbf{2 0 0 9} \\
\text { US\$ }\end{array}$ & $\begin{array}{c}\text { per capita } \\
\mathbf{2 0 0 9} \\
\text { millions }\end{array}$ & $\begin{array}{c}\text { US\$ } \\
\text { inequality } \\
\text { (year) }\end{array}$ & $\begin{array}{c}\text { At } \\
\text { birth }\end{array}$ & $\begin{array}{c}\text { At age } \\
\mathbf{6 5}\end{array}$ \\
\hline Australia & 877 & 39,918 & $30.5(2006)$ & 4 & 6 \\
\hline Canada & 1,276 & 37,873 & $32.1(2005)$ & 9 & 8 \\
\hline $\begin{array}{l}\text { New } \\
\text { Zealand }\end{array}$ & 125 & 29,149 & $36.2(1997)$ & 13 & 14 \\
\hline $\begin{array}{l}\text { United } \\
\text { Kingdom } \\
\text { (UK) }\end{array}$ & 2,173 & 35,151 & $34.0(2005)$ & 17 & 20 \\
\hline $\begin{array}{l}\text { United } \\
\text { States of } \\
\text { America } \\
\text { (US) }\end{array}$ & 14,044 & 45,087 & $45.0(2007)$ & 25 & 22 \\
\hline
\end{tabular}

Sources and notes:

Gross Domestic Product (GDP) and GDP per capita: Estimated, expenditure approach, US \$, current prices, current PPPs, OECD statistics extracted February 2011.

Gini index for distribution of family income. Takes value zero if perfect equality; 100 if income were distributed with perfect inequality. Extracted from CIA World Factbook February 2011.

Total population life expectancy ranking (unweighted average of male and female) from OECD (OECD 2010). 
Comparison with European countries and other geographically closer countries was considered but not pursued. European countries such as Denmark, Norway and Sweden, as well as Japan offer good data that has been extensively analysed. These countries are demographically interesting in part because of high life expectancy. Japan leads the world on life expectancy at birth, and Norway and Sweden rank in the top ten of the OECD. However, these countries have less history to share with New Zealand than does Britain, and are more ethnically homogenous than New Zealand. Further, current projection methods have been compared for European Union (EU) countries by Lutz (2009), so new insights can be obtained by investigations outside of EU countries. Finally, no EU country other than the UK undertakes detailed actuarial analysis by which additional insight could be obtained.

The comparison with the United Kingdom was the basis for New Zealand's first mortality studies. The first compilation of New Zealand mortality rates for a continuous period was by George Leslie in 1895, who found "very favourable - so favourable indeed as to be remarkable" mortality rates among non-Māori compared to select sub-groups within England and Wales (Leslie 1895 p. 7). Lessof (1949) favourably compared the infant mortality at ages between 4 weeks and under 12 months of non-Māori in New Zealand with that in England and Wales. Early studies such as these were limited by excluding data on Māori mortality. However, they provide some insight into the development of settler mortality in New Zealand, and establish the precedent of comparing with British mortality.

The hypothesis is that having shared some social and genetic history, these British settler countries have followed similar mortality trajectories in the past so that differences identified by comparative analysis would be 
informative. The mortality projections made in each country can therefore add insights to inform the others. Specifically, the mortality projections of the other countries can help to critique New Zealand's in the context of findings from the comparative analysis of historical mortality.

\subsection{Selection of statistics}

The first section in this chapter cautioned against the common practice of relying on life expectancy - and particularly period life expectancy at birth - as the single measure to compare mortality levels and trends across countries and over time. For this analysis, a range of statistics is selected to provide a more detailed empirical base of mortality data. This section explains the rationale for the selection.

\section{Levels of mortality}

Period life expectancy at birth is a simple summary of mortality rates across a population at a point in time. It therefore provides a useful summary comparison of mortality across countries. Because of the emphasis in this thesis on superannuation policy, and the importance to that of later life mortality, life expectancy at age 65 and age 80 will be compared between selected countries, as well as life expectancy at birth.

However, as discussed in section 3.1, the life expectancy measure can hide critical detail on what has happened or is happening in mortality rates at individual ages. Cohort life expectancies are not produced as frequently as period life expectancies. The Human Mortality Database contains past mortality rates and life expectancies on a period basis, but only past mortality 
rates on a cohort basis (which are just a reorganising of period mortality rates into generational labels). Cohort life expectancies for current and future generations require assumptions of future mortality rates and are generally produced as part of mortality projections. These are therefore considered in Chapter 5, while to supplement the analysis of period life expectancy here, actual mortality rates will be additionally examined, as well as past trends in those rates.

Demographers and actuaries use mortality rates, either $q_{x}$ or $m_{x}$, as the smallest unit for measuring the probability of death (for definitions see section 1.5). Both q-type and m-type mortality rates can be used for comparison of past trends or projecting future trends. Here, $q_{x}$ are compared across countries. These are the most convenient consistent mortality rates available from each country's life tables. In order to compare the level of mortality rates $\mathrm{q}_{\mathrm{x}}$ across all ages (x) direct standardisation is used (Rowland 2006 p. 125). This technique converts each country's overall mortality rate for an age group to a comparable number as if all had the same age profile, thus removing any differences in numbers of deaths from population number differences at each age.

\section{Trends in mortality rates}

In any comparison of mortality rates, different patterns may be seen along different axes. An 'age effect' is noted where mortality rates in a specific period change in a defined way with increasing age. A 'period effect' defines where age-specific mortality rates change at about the same pace over time. A 'cohort' effect describes the situation where members of a cohort sharing the same birth period share a particular mortality trend as they age. As Hobcraft 
et al (1982) point out, age-period-cohort effects are the core of the interpretation of demographic data, but rarely provide a unique explanation. Often these effects describe observed variations rather than causes; for example, it is not the period of time itself influencing mortality rates but something that happened during that period. Further, it is usually a matter of opinion which of the trends is predominant. For example, if a period effect has different impact on different ages, then it may appear to be a cohort effect but cannot be proven either way. Therefore, any comparison from different countries of trends in a demographic variable like $q_{x}$ for defined ages and period may not provide a definitive explanation for the cause of the trends and the relative position of the countries.

The presence of, and explanation for, cohort effects have controversially dominated the analysis of recent national mortality trends. As discussed in section 2.2, the 'golden cohort' born between 1923 and 1940 in the UK (or, more precisely, much of the analysis has used data for the region England and Wales) has consistently experienced more rapid improvement in mortality rates throughout life than those born before or after. This observation has been debated most prominently among actuaries. Given actuarial input to the national population projections in the UK, and the timing of the discovery of a golden cohort near pension age coinciding with a pension policy focus (Pensions Commission 2004, 2005), the golden cohort made its mark on national population projections. There was a rapid increase in the optimism of the mortality assumption for the UK's national population projections, including an assumption that the golden cohort would continue to keep its mortality improvement advantage until death (Dunnell 2008; Gallop 2008; ONS 2006; Willets 2004). 
Andreev and Vaupel (2005) sought patterns in mortality rates by age and time since the 1950s for various countries, and identified a cohort effect superimposed on a predominantly period pattern for England and Wales. They also found cohort effects in Austria, Denmark, France, Italy, Japan, the Netherlands, Switzerland and West Germany and predominantly period effects in the USA and Canada. In Australia and New Zealand they identified 'age shifting' effects where over time the highest rates of mortality improvement shift from younger to older ages. O'Connell and Dunstan (2009) compared New Zealand's mortality rates with those of England and Wales for periods since 1961 to conclude that a golden cohort was apparent in both countries. However, although both claims to a golden cohort were debatable, that of England and Wales appeared the stronger.

Murphy (2010) has suggested that too much emphasis has been placed on cohort effects in explaining population-level mortality trends and argued for more thought to be given to what macro-level factors cause mortality trends, focusing on the disadvantaged groups rather than those with high mortality improvement rates. However, Murphy's analysis did not deny the presence of the cohort patterns in data from Japan and England and Wales, nor did it derive new explanations for the causes. Further, given the cohort's current age, the unexpected longevity has an immediate current and negative economic impact on the cost of pension funds and public pensions so there is a compelling policy rationale for the analytic emphasis on the golden cohort. Therefore, despite the controversy over the relative significance of apparent cohort effects, they merit consideration alongside age and period effects in this comparative mortality analysis. 
The literature on cohort effects in mortality trends suggests a framework for comparative analysis across countries. In all the above-mentioned papers, the measure of mortality change over time used is the annual rate of change in the mortality rate, shown here for a q-type rate:

$$
\mathrm{QXI}=1-\frac{q_{x, t}}{q_{x, t-1}} \text { for age } \mathrm{x} \text { at time } \mathrm{t}
$$

This measure has not been defined in the literature, but is here defined as QXI, to denote ' $\mathrm{q}_{\times}$improvement'. This is because the prevailing trend in all selected countries for the majority of time periods and ages has been mortality improvement, which gives a positive value for QXI as defined. A negative value for QXI denotes a mortality rate at age $\mathrm{x}$ increasing over the time period $\mathrm{t}$, that is, worsening mortality. An m-type or q-type QXI is the basis for projecting mortality in national population projections, as discussed further in section 5.1. In this study, following the international comparison of the levels of mortality rates, a comparison of QXI over time for each age $\mathrm{x}$ is made to examine trends in mortality and age-period-cohort effects.

In many papers investigating QXI, Lexis maps are the preferred method. A Lexis map plots the derivative of smoothed age-specific mortality rates (or their logarithms) with age and calendar time on two axes, so that a birth cohort is represented along the diagonal. The plots are therefore a continuous representation of the above equation for QXI. The rate of mortality change is coloured to show visually age-period-cohort defined areas of greatest mortality improvement, and a golden cohort is shown by a strongly-coloured diagonal. However, other papers have used simple tables of QXI. O'Connell and Dunstan (2009) compared QXI of New Zealand and England and Wales, 
following Gallop (2008), noting that the greater detail of Lexis maps did not yield more valuable insights. For the purpose of identifying differences in national population mortality trends that are inevitably unable to be fully explained by underlying cause, the greater complexity of Lexis maps does not appear justified. Further, while Lexis maps use smoothed data that prevents year by year variations disturbing a view of the long-term trend, an analysis of QXI uses smoothed mortality rates from life tables which usually represent more than one year's data. Therefore the convenience and simplicity of tabulated numeric QXI are preferred here, and the methodology of O'Connell and Dunstan (2009) is extended to the selected comparator countries.

\section{Age at death measures}

The assertion that life expectancy at birth is not an adequate measure of mortality trends for a population in which the indicator approaches 80 years (Olshansky et al. 1990) applies to the selected countries in this analysis. Following the suggestion that trends in life expectancy at birth, the median and adult modal ages at death together provide a basis for better explanation of trends than any one of those measures alone (Canudas-Romo 2010), all these measures will be analysed for each of the countries. Median and modal ages at death are identified from further columns in the life table. In a period life table, the probability of dying aged $x\left(q_{x}\right)$ is applied to the hypothetical number of lives left at age $x, 1_{x}$. The starting number of lives (radix of the table), 10 , is set at an arbitrary number, usually 100,000 . Then it follows that the number of deaths at each age $x$ for that radix is

$$
d_{x}=l_{x} \cdot q_{x}
$$


The median age at death is the age at which the number of surviving lives $1_{x}$ falls below half of the radix. The adult modal age at death is the age at which the highest number of deaths $d_{x}$ occurs among adult ages. It is necessary to stipulate adult ages so as to exclude the case where there may be a higher number of deaths at age zero (newborn). Formulae to enable these two measures to be calculated with decimal point precision are given in Canudas-Romo (2010) Appendix A:

Median age at death at time $t=$

$$
x+\frac{\left(0.5-l_{x, t}\right)}{\left(l_{x+1, t}-l_{x, t}\right)}
$$

where $x$ and $x+1$ form the age interval in which $1_{x}$ crosses half of the radix of the life table at time $t$, radix $=1$.

Modal age at death at time $\mathrm{t}=$

$$
x+\frac{\left(d_{x, t}-d_{x-1, t}\right)}{\left(d_{x, t}-d_{x-1, t}\right)+\left(d_{x, t}-d_{x+1, t}\right)}
$$

where $x$ is the age with the highest number of deaths, $x>0$.

Because mortality rates at the oldest ages are based on smaller samples, smoothing and approximation becomes more important. Therefore, comparisons of $\mathrm{q}_{\mathrm{x}}$ and QXI at older ages may be distorted by methods used to smooth. Some analysis is therefore truncated at older ages, but analysis of life expectancy at older ages and of modal ages at death yield further insight on mortality at older ages. 


\subsection{Selection of mortality projections}

Reports on past mortality trends and on future mortality projections from both demographic and actuarial agencies in the selected countries provide data and background context for what follows in the next two chapters. These source materials are referenced in Table 3.7. Each set of projections is the latest available at the time of analysis for this thesis (2009-10) so they form a set contemporary with the projections of New Zealand mortality available at the time of the survey of subjective lifespans.

Each of the mortality projections considered from the sources in Table 3.7 uses the extrapolation techniques discussed in section 2.3. However, the details of each method vary. Different specific techniques or formulae for extrapolation are used. Extrapolation, although essentially formulaic, requires judgement about, for example, how long a past trend should be assumed to carry forward, and how far it should be allowed to trend before levelling off. In order to decide on the parameters for extrapolation formulae, agencies supplement the information from past trends in different ways. For example, the assumptions for the projections by the UK's Office for National Statistics are influenced by the views of an expert panel on what future mortality trends might be. However, none of the agencies develop a biodemographic rationale for adjustments to pure extrapolation, or give a rationale based on international comparisons, beyond very general statements. 
Table 3.7: Main demographic and actuarial sources of national population projections in selected countries

\begin{tabular}{|c|c|c|}
\hline & Demographic & Actuarial \\
\hline Australia & $\begin{array}{l}\text { Population Projections, } \\
\text { Australia } 2006 \text { to } 2101 \\
\text { Australian Bureau of } \\
\text { Statistics (ABS) } \\
\text { ABS (2008) }\end{array}$ & $\begin{array}{l}\text { Australian Life Tables } \\
2005-7 \\
\text { Australian Government } \\
\text { Actuary (AGA) } \\
\text { AGA (2009) }\end{array}$ \\
\hline Canada & $\begin{array}{l}\text { Population Projections for } \\
\text { Canada, Provinces and } \\
\text { Territories } 2009 \text { to } 2036 \\
\text { Statistics Canada (2010) }\end{array}$ & $\begin{array}{l}\text { Canada Pension Plan } \\
\text { Mortality Study } \\
\text { Actuarial Study No. } 7 \\
\text { Office of the Chief Actuary } \\
\text { (OCA) } \\
\text { OCA (2009) } \\
\text { (Note 1) }\end{array}$ \\
\hline New Zealand & $\begin{array}{l}\text { National Population } \\
\text { Projections: } 2009 \text { (base)- } \\
2061 \\
\text { Statistics New Zealand } \\
\text { (2009a) }\end{array}$ & $\begin{array}{l}\text { None } \\
(\text { Note 2) }\end{array}$ \\
\hline $\begin{array}{l}\text { United } \\
\text { Kingdom } \\
\text { (UK) (Note 3) } \\
\end{array}$ & \multicolumn{2}{|c|}{$\begin{array}{l}\text { 2008-based National Population Projections } \\
\text { Office for National Statistics (ONS) } \\
\text { ONS (2009) }\end{array}$} \\
\hline $\begin{array}{l}\text { United States } \\
\text { of America } \\
\text { (USA) }\end{array}$ & $\begin{array}{l}2008 \text { National Population } \\
\text { Projections } \\
\text { US Census Bureau (2008) }\end{array}$ & $\begin{array}{l}2010 \text { OASDI Report (The } \\
2010 \text { Annual Report of the } \\
\text { Board of Trustees of the } \\
\text { Federal Old-Age and } \\
\text { Survivors Insurance and } \\
\text { Federal Disability } \\
\text { Insurance Trust Funds) } \\
\text { OASDI Board of Trustees } \\
\text { (2010) } \\
\text { (Note 4) }\end{array}$ \\
\hline
\end{tabular}

Notes for Table 3.7:

Note 1: The Canadian Pension Plan Mortality Study gives mortality data for Canada less Québec.

Notes continue on following page. 
Notes for Table 3.7, continued:

Note 2: The Government Actuary in New Zealand has a regulatory role for superannuation schemes and KiwiSaver, but does not produce mortality data or population projections.

Note 3: Population projections in the UK have a strong actuarial influence. The Government Actuary's Department (GAD) was responsible for producing the UK's official national population projections from 1954 until 2006. Responsibility for the production of the official national population projections and associated demographic data then transferred to the ONS. A specialist unit for mortality data is staffed jointly by demographers and actuaries.

Note 4: The population base for the OASDI report comprises beneficiaries and future beneficiaries of the OASDI program.

Further, none of the sources give sufficient detail about the methods used or judgements made to allow a reader to reproduce the calculations or fully critique the assumptions underlying the results. The number of assumptions and complexities by age, gender and time period would be too demanding. However, it is not necessary for this purpose to replicate each calculation or compare the methodology of each projection in detail. Here, the comparison of New Zealand's mortality projections to those of other countries can be described by a comparison of assumptions and results, and by setting these in context with the comparison of past mortality trends in the selected countries.

\section{Focusing the choice of projections for comparison}

For this study, it is preferred to use cohort measures to compare future estimates of population mortality across countries. Cohort life expectancy is more relevant to meaningful individual lifespan prospects, rather than period measures, as discussed in section 3.1. The actuarial agencies of Australia, Canada and the US publish projected future cohort life expectancy, whereas 
the statistical agencies of those countries only publish period measures. The UK's statistical agency publishes cohort measures. Statistics New Zealand publishes official population projections using period life expectancy measures, but has made available estimates of future cohort life expectancy consistent with the assumption scenarios of the official projections (obtained through personal communication with Statistics New Zealand November 2009).

There is often more useful detail on the assumptions for and results of mortality projections in the actuarial agency reports than in the statistical agency projections. For example, in Australia, the projections from the Australian Bureau of Statistics are made by assuming a simple future rate of increase in life expectancy at birth which is a marked exception from the usual practice of extrapolating age-specific mortality rates. Generally, estimates of future life expectancy are published only in summary for few future years by statistical agencies, whereas the actuarial reports present or make freely available more data. Mortality is only one part of the total population projections carried out by the statistical agencies, but is often more important in the work of the actuarial agencies, especially if the projections are carried out for the national pension or social security plan, as they are in Canada and the US. Because the most useful detailed data using cohort measures is available in the actuarial projections for Australia, Canada and the US, it is these projections (that is, sourced from the right hand column in Table 3.7) that are compared with the actuarially-influenced projections of the UK and the official projections from Statistics New Zealand. 
A potential disadvantage of this approach is that the actuarial mortality projections for Canada and the US are made for a different purpose and do not cover the entire national population that was the basis for the international mortality comparison in Chapter 4. However it does not appear to be the case that distinctive mortality characteristics of these sub-populations would distort the analysis here. The actuarial mortality projections for the OASDI report in the US (see Table 3.7) are made using National Center for Health Statistics data on deaths and Census estimates of population, and so reflect national mortality characteristics and for practical purposes can be taken to cover the total population (OASDI Board of Trustees 2010 p. 79). The actuarial projection in Canada is for the Canada Pension Plan in Canada less Québec. Mortality improvement rates which formed the basis of the projections were checked for consistency with national data (OCA 2009 p. 27). Canada less Québec is estimated to comprise 77 per cent of the national population in 2009 (Statistics Canada 2010 Table 8). Nearly all other provinces or territories within Canada differ on period life expectancy in 2006 and 2036 for males and females from the national average by greater amounts than Québec (Statistics Canada (2010) Table 1.3). The actuarial mortality projections of Canada and the US have been compared with the national mortality projections of the UK before for the purpose of comparing methods and population mortality patterns (Gutterman 2008). This present study uses more recent data, adds national mortality projections from the Australian Government Actuary and Statistics New Zealand, and extends the analysis to question the source of differences - the assumptions made by the different agencies. 


\subsection{Conclusions}

Literature reviewed in Chapter 2 raised the prospect of individual and population average lifespan being underestimated in an era of continuing mortality improvement, as a consequence of the methods used to produce estimates of future longevity. This chapter identifies further reasons why lifespans are underestimated: the most common measure of life expectancy is constructed without taking age- and time-dependent mortality change into account, yet is routinely misrepresented as if it were an indicator of what individuals should expect their lifespan to be. Further, life expectancy (and especially period life expectancy at birth) is commonly used to show time trends of population mortality or lifespan, yet is increasingly inadequate for that purpose especially in high life expectancy environments like New Zealand. Once the construction of period life expectancy is understood, these results are intuitive, as is the superiority of cohort life expectancy as an indicator of realistic lifespans and mortality trends. Yet the use and analysis of cohort life expectancy lags behind that of period life expectancy.

This thesis develops a view on plausible future average lifespans in New Zealand so that actual expectations can be assessed against a likely reality. This is the basis for understanding whether longevity risk exists. In this study, a framework is developed to review existing mortality projections for plausibility. This framework builds on the understanding of the flaws in common measures of lifespan, on the encouragement to use comparisons of mortality trends between-countries and within-country as inputs to projecting future mortality, and on learning from emerging demographic theories of mortality variance. 
The first two parts of this framework collect empirical evidence on New Zealand's past and projected future mortality compared to other countries, to draw inferences by comparison. The comparative analysis is made for mortality experience since the 1960s for the selected British settler countries. The countries were selected because of the social and ancestral similarities with New Zealand, as well as each other country providing robust actuarial data, the analysis of which is new to New Zealand. Significant mortality issues within New Zealand are also covered insofar as they affect the extrapolation of mortality projections. The time period for analysis follows other studies to focus on the decades since 1960 so that a modern history is examined.

The evidence to be collected and interpreted involves more than the usual single measure of life expectancy. The measures used here supplement the flawed period life expectancy measure by additionally examining the level of age-specific mortality rates and their annual rate of improvement. The contribution of younger cohorts to future mortality is considered by specific analysis of mortality improvement rates by age and cohort. Older age mortality is additionally assessed by the use of median and adult modal ages at death.

The analytic framework designed here to enhance interpretation of the extrapolative method used in New Zealand's mortality projections is an original approach which builds on previous literature in order to develop a new view of New Zealand's likely future mortality. 


\section{Chapter 4: New Zealand mortality in international context}

This chapter gives the empirical results from the first part of the analytic framework set out in the previous chapter: comparative analysis of New Zealand's and selected countries' past mortality trends. Each section covers a different mortality indicator with the concluding section highlighting the notable features of New Zealand mortality.

\subsection{Level of mortality rates}

The mortality rates for males at the beginning of the comparison period are shown in Figure 4.1 and for the end of the period in Figure 4.2. Equivalent figures for females follow. For all life table data in this chapter, the statistics were obtained from the life tables in the Human Mortality Database (HMD; www.mortality.org) except for New Zealand data which was taken directly from life tables published by Statistics New Zealand (downloaded from www.stats.govt.nz). This was for ease of use; the data is consistent between the two sources. HMD contains mortality rates for each calendar year, taken from published life tables which may span a longer period. Thus "1961" and "2006" are used to denote the entries from those years from HMD, but the New Zealand data is from the period life tables of 1960-62 and 2005-7 respectively. 
Figure 4.1: Mortality rate at age $x\left(q_{x}\right)$ for ages 0 to 100 years for selected countries, logarithmic scale, males, 1961

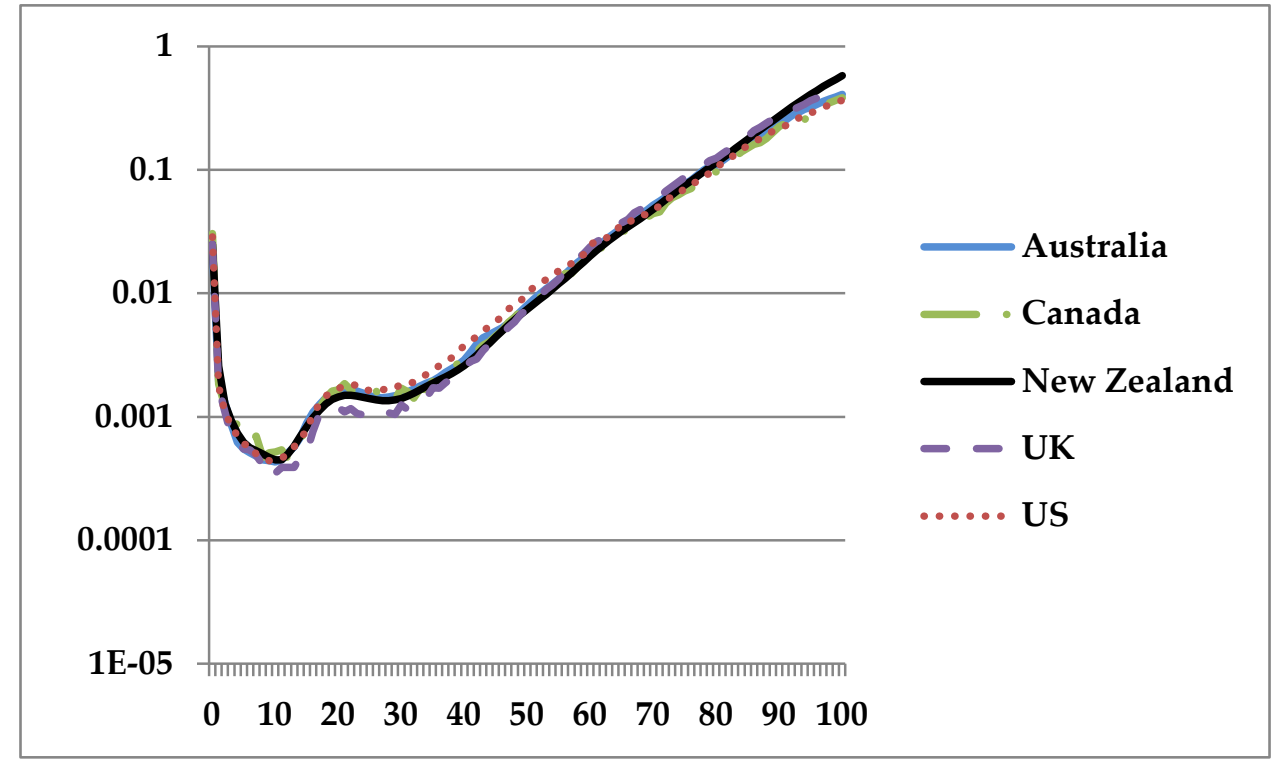

Source: New Zealand mortality rates from Statistics New Zealand life tables. Other mortality rates from Human Mortality Database life tables (downloaded August 2010).

Figure 4.2: Mortality rate at age $x\left(q_{x}\right)$ for ages 0 to 100 years for selected countries, logarithmic scale, males, 2006

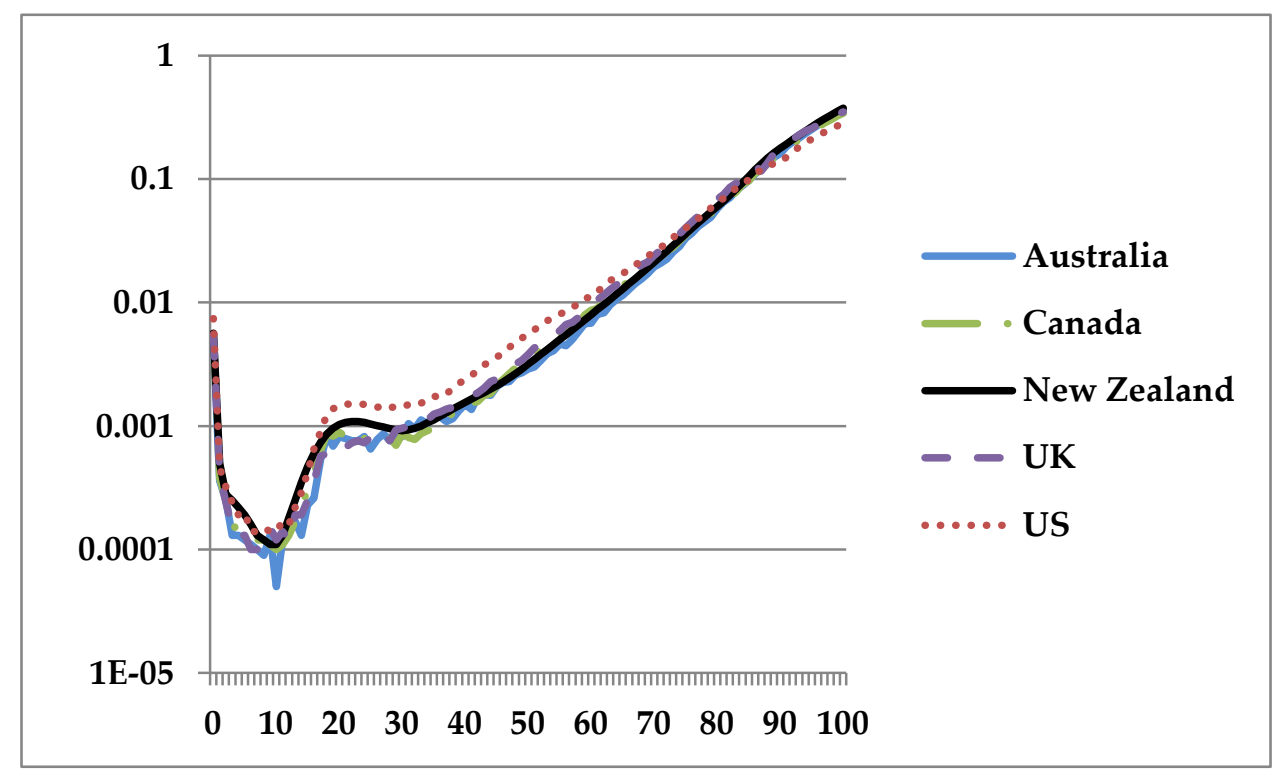

Source: As Figure 4.1. 
Figure 4.3: Mortality rate at age $x\left(q_{x}\right)$ for ages 0 to 100 years for selected countries, logarithmic scale, females, 1961

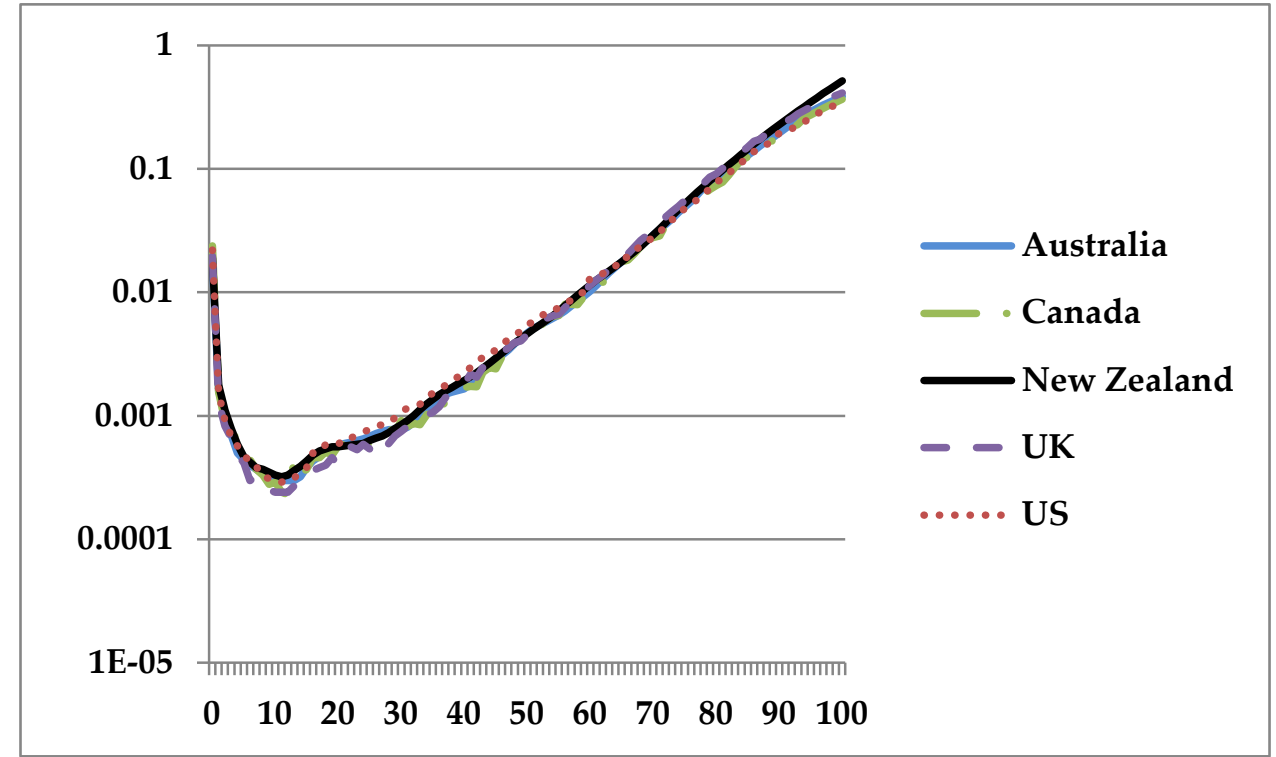

Source: As Figure 4.1.

Figure 4.4: Mortality rate at age $x\left(q_{x}\right)$ for ages 0 to 100 years for selected countries, logarithmic scale, females, 2006

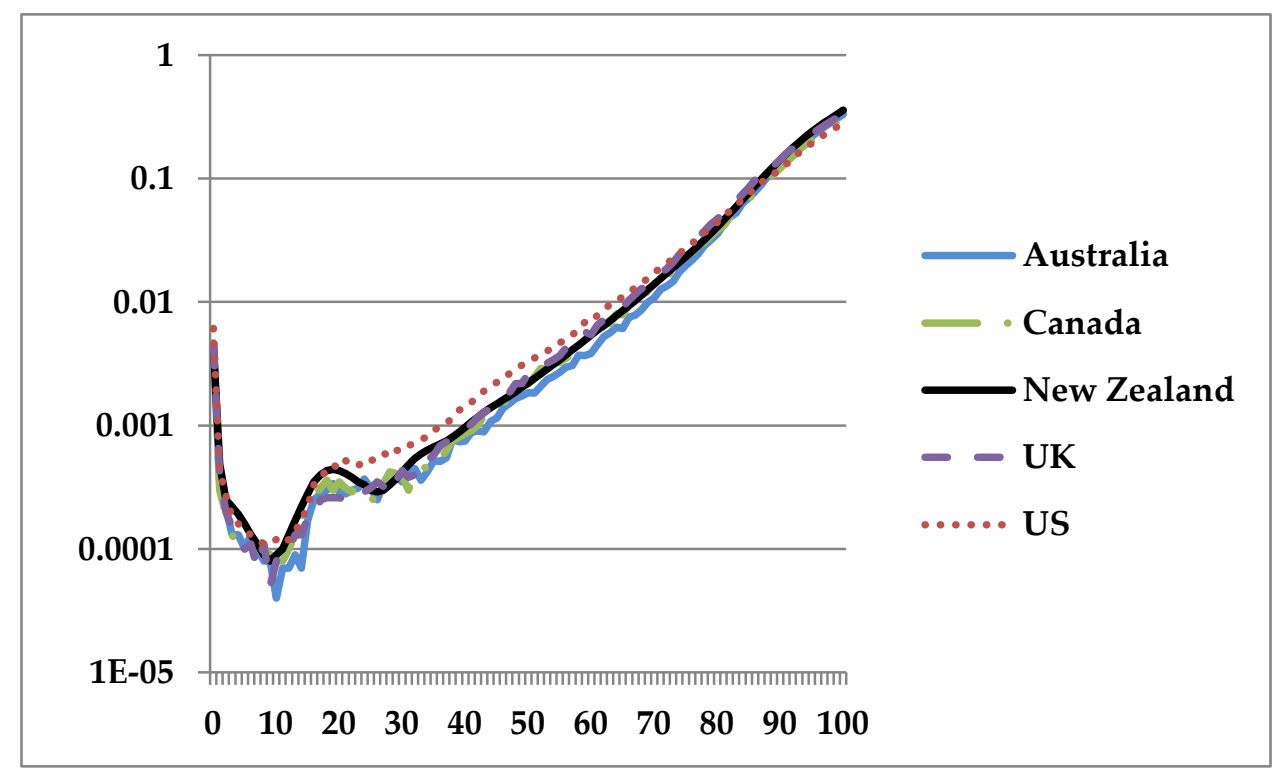

Source: As Figure 4.1. 
The selected countries have similar levels of age-specific mortality rates, similar patterns by age and gender and similar trends over time. The shape of the graphs is the classic one for all developed countries: the mortality rate at birth is higher than that at age 1, then the curve falls rapidly to low levels for young children. It rises steadily as age increases interrupted only by the 'accident hump' of higher mortality at late teen ages and twenties, especially in males. A sharper accident hump appears for females around age 20 in 2006 compared to 1961 for all countries because of a more rapid fall in mortality rates either side of the apparent hump. Female mortality rates are lower than those for males at all ages and both time periods. From 1961 to 2006 mortality rates have fallen. Differences between the countries appear to have widened slightly by 2006 compared to 1961 . New Zealand's mortality rates are in the middle of the group of countries. There is little apparent difference between countries except that US mortality rates are noticeably higher (worse) from age 20 until age 70 when they cross over to become lower (better).

Table 4.1 shows standardised mortality ratios for the same two periods. The all-ages figures (up to age 80 shown here) confirm that New Zealand's mortality is in the middle of the selected group of countries. Against the UK and Canada, the relative all-ages position of New Zealand has changed little over the last forty-five years. New Zealand had in 2006 better mortality than the US and UK for both males and females. Male mortality in New Zealand is on a par with that in Canada. New Zealand mortality is worse than Canada for females, and for both genders compared to Australia. The improvement in Australia's relative position between 2006 and 1961 is striking. 
New Zealand's improved position has been especially marked against the US at all ages except for females aged 1-20. New Zealand's relative mortality appears to have improved at ages over 40 but worsened at younger ages, compared to both Canada and the UK, especially for males. Compared to Australia, New Zealand's relative mortality has worsened at all ages except for males aged 0 to 20 . The mortality of females at ages 1-20 appears to have worsened in New Zealand considerably from 1961 to 2006 relative to all the comparator countries. The mortality of New Zealand newborns (age 0) appears to be similar or slightly worse in the 2006 comparison with Australia, Canada and the UK relative to 1961 but improved compared to the US. Newborn mortality requires detailed examination within the first year of life, outside the scope of this study.

Table 4.1: Directly standardised mortality ratio in year shown, standardised to New Zealand 2006 population

\begin{tabular}{|lllll|}
\hline 1961 & & & & \\
Male & Australia & Canada & UK & US \\
\hline Age 0 & $89 \%$ & $122 \%$ & $101 \%$ & $115 \%$ \\
Age 1-20 & $99 \%$ & $103 \%$ & $80 \%$ & $98 \%$ \\
Age 21-40 & $108 \%$ & $106 \%$ & $84 \%$ & $128 \%$ \\
Age 41-60 & $110 \%$ & $104 \%$ & $108 \%$ & $128 \%$ \\
Age 61-80 & $105 \%$ & $91 \%$ & $116 \%$ & $99 \%$ \\
Age 0-80 & $105 \%$ & $97 \%$ & $111 \%$ & $108 \%$ \\
& & & & \\
Female & Australia & Canada & UK & US \\
\hline Age 0 & $92 \%$ & $125 \%$ & $103 \%$ & $116 \%$ \\
Age 1-20 & $92 \%$ & $92 \%$ & $76 \%$ & $94 \%$ \\
Age 21-40 & $94 \%$ & $90 \%$ & $87 \%$ & $116 \%$ \\
Age 41-60 & $94 \%$ & $93 \%$ & $99 \%$ & $111 \%$ \\
Age 61-80 & $96 \%$ & $91 \%$ & $107 \%$ & $94 \%$ \\
\cline { 2 - 5 } Age 0-80 & $95 \%$ & $93 \%$ & $103 \%$ & $99 \%$ \\
\hline Table continues, with notes, on following page. & \\
\hline
\end{tabular}


Table 4.1: Directly standardised mortality ratio in year shown, standardised to New Zealand 2006 population, continued

\begin{tabular}{|lllll|}
\hline Male & & & & \\
Age 0 & $93 \%$ & $98 \%$ & $98 \%$ & $132 \%$ \\
Age 1-20 & $71 \%$ & $81 \%$ & $71 \%$ & $117 \%$ \\
Age 21-40 & $88 \%$ & $85 \%$ & $94 \%$ & $148 \%$ \\
Age 41-60 & $90 \%$ & $106 \%$ & $117 \%$ & $162 \%$ \\
Age 61-80 & $91 \%$ & $101 \%$ & $114 \%$ & $118 \%$ \\
Age 0-80 & $90 \%$ & $100 \%$ & $112 \%$ & $130 \%$ \\
& & & & \\
Female & Australia & Canada & UK & US \\
\hline Age 0 & $91 \%$ & $104 \%$ & $103 \%$ & $136 \%$ \\
Age 1-20 & $69 \%$ & $76 \%$ & $68 \%$ & $97 \%$ \\
Age 21-40 & $82 \%$ & $86 \%$ & $92 \%$ & $145 \%$ \\
Age 41-60 & $79 \%$ & $97 \%$ & $108 \%$ & $141 \%$ \\
Age 61-80 & $83 \%$ & $97 \%$ & $113 \%$ & $120 \%$ \\
Age 0-80 & $82 \%$ & $96 \%$ & $110 \%$ & $125 \%$ \\
\hline
\end{tabular}

Source: New Zealand mortality rates from Statistics New Zealand life tables. Other mortality rates from Human Mortality Database life tables (downloaded August 2010).

Notes:

Table 4.1 shows mortality rates $\mathrm{q}_{\mathrm{x}}$ to age 80 from period life tables for each country standardised to the New Zealand 2006 Estimated Resident Population (from Statistics New Zealand), as a ratio to that for New Zealand mortality rates. The standardised measure averages across ages to yield one measure for the population. The use of the same population for comparison removes the effect of different population age structures between countries and time periods. A ratio greater than 100 per cent implies New Zealand mortality was lighter (better) than the compared country and a ratio of less than 100 per cent means that New Zealand mortality was worse. Analysis was cut off after age 80 as mortality rates above that age are less stable. 


\subsection{Life expectancy}

Figure 4.5 compares period life expectancy at birth for 1961 for males and females in the selected countries and Figure 4.6 does so for 2006. Note that the scales on these graphs are truncated to allow focus on the differences in value between countries. As period life expectancy is calculated using mortality rates at each age at that point in time, it is a summary measure of all ages mortality. The figures confirm that the selected countries have similar levels of mortality, and that New Zealand is a relatively healthy country compared to other settler nations. In 1961, New Zealand mortality appeared better than all selected countries for male life expectancy at birth, although only slightly (by 0.04 of a year) compared to Canada. For females, New Zealand mortality was slightly better than that in both the UK and US but more significantly behind that in Australia and Canada. Since then, life expectancy at birth in Australia markedly improved to take first place for both males and females. Compared to Canada, the relative mortality position of female New Zealanders did not change and there was a slight deterioration in that of male New Zealanders. Life expectancy in the US lags throughout the period. 
Figure 4.5: Period life expectancy at birth for selected countries, in years, 1961

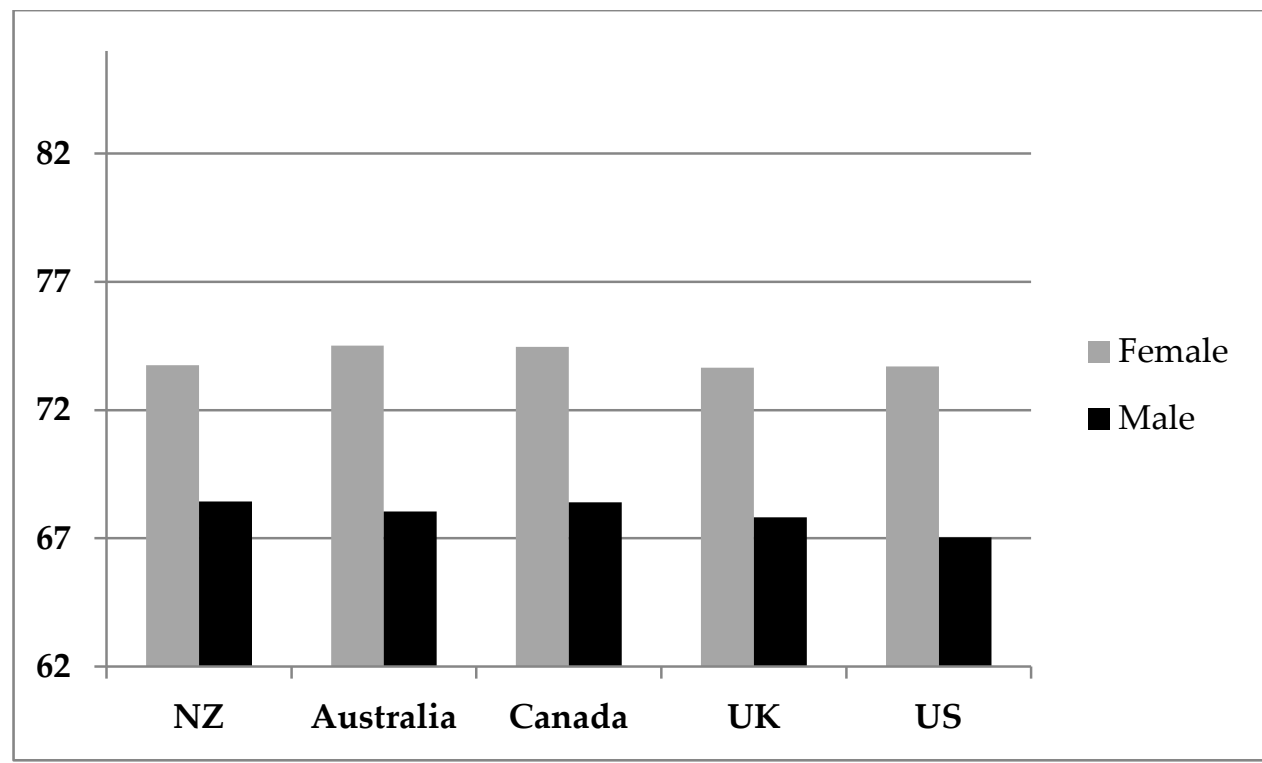

Source: New Zealand data from Statistics New Zealand life tables. Other mortality rates from Human Mortality Database life tables (downloaded August 2010).

Figure 4.6: Period life expectancy at birth for selected countries, in years, 2006

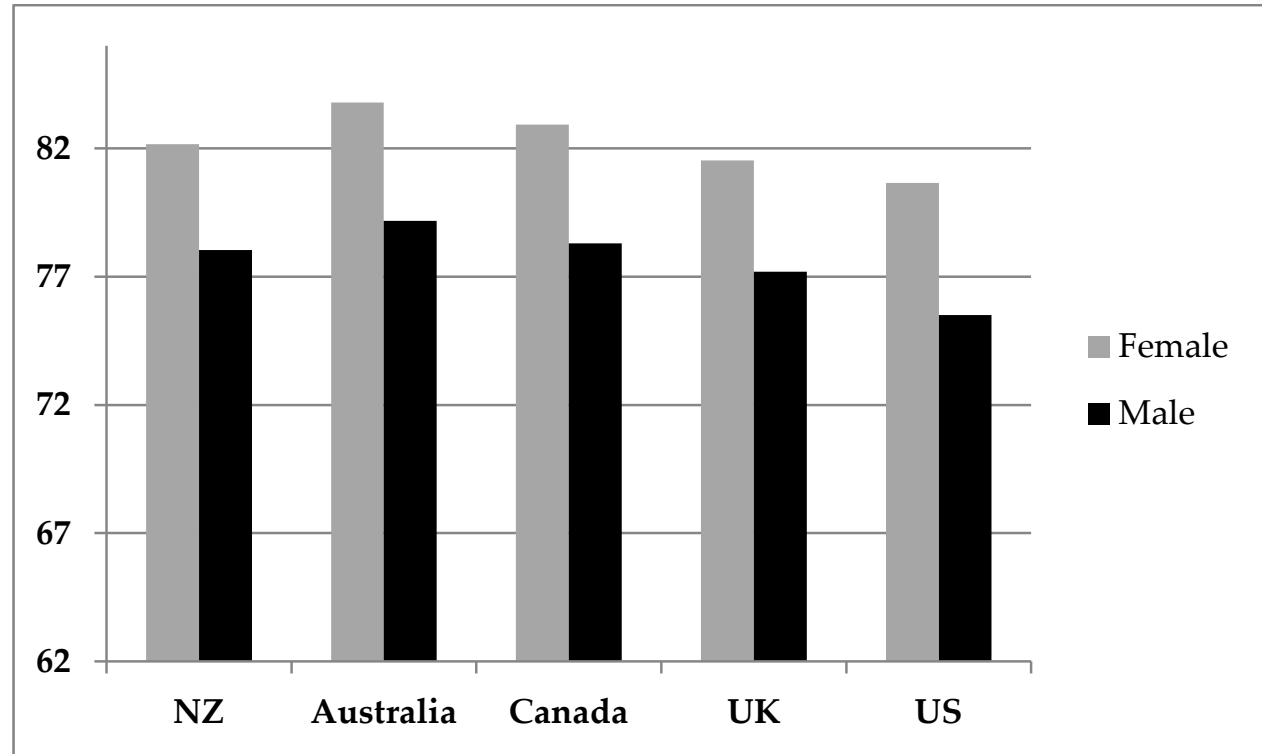

Source: As Figure 4.5. 
Further insights on age-related changes in the comparison of mortality can be seen by looking at life expectancy at ages 65 and 80 . These measures incorporate the life table mortality rates from those ages until the table ends into one number, therefore smoothing out potential distortions in mortality rates at the highest ages caused by small numbers. Table 4.2 shows the life expectancy measures, including life expectancy at birth for completeness, with additional statistics on the difference between each country and New Zealand.

The ranks of New Zealand life expectancy measures have not changed over the period, with two exceptions. The first is the rank of male life expectancy at birth which fell from number 1 (highest life expectancy, best mortality) in 1961 to 3 in 2006. This was due to relative improvements for both Canada and Australia, while the gap to the UK changed little, and mortality in the US worsened relatively. The second is the increase in rank for female life expectancy at age 65 from 4 to 3 . This is due to the changing position of US life expectancy at age 65 over the period, from rank 2 in 1961 to rank 4 in 2006. The 1961 rank is anomalous for relative US mortality at younger ages. By 2006 the effect on mortality at older ages has lessened, so that the rank of New Zealand's life expectancy at age 65 returns to the rank for life expectancy at birth. However, the effect is still present in life expectancy at age 80 so that for the US ranks best for females in 2006, and third for males. This pushes New Zealand into fourth place. 
Table 4.2: New Zealand period life expectancy at birth, at age 65 and at age 80 compared to selected countries, 1961 and 2006

\begin{tabular}{|c|c|c|c|c|c|c|}
\hline $\begin{array}{l}\text { Period lif } \\
\text { measure }\end{array}$ & expectan & $y$ in ye & rs, and rar & k of $\mathrm{Ne}$ & w Zealand & \\
\hline & At birth & & At age 65 & & At age 80 & \\
\hline 1961 & Females & Males & Females & Males & Females & Males \\
\hline Australia & 74.5 & 68.1 & 15.9 & 12.6 & 7.1 & 5.8 \\
\hline Canada & 74.5 & 68.4 & 16.3 & 13.6 & 7.3 & 6.3 \\
\hline NZ & 73.8 & 68.4 & 15.5 & 12.8 & 6.4 & 5.5 \\
\hline UK & 73.7 & 67.8 & 15.1 & 11.9 & 6.3 & 5.2 \\
\hline US & 73.7 & 67.0 & 16.2 & 13.1 & 7.2 & 6.1 \\
\hline NZ rank & 3 & 1 & 4 & 3 & 4 & 4 \\
\hline 2006 & & & & & & \\
\hline Australia & 83.8 & 79.2 & 21.7 & 18.6 & 10.0 & 8.3 \\
\hline Canada & 82.9 & 78.3 & 21.3 & 18.2 & 10.2 & 8.3 \\
\hline $\mathbf{N Z}$ & 82.2 & 78.0 & 20.6 & 18.0 & 9.5 & 8.0 \\
\hline UK & 81.5 & 77.2 & 20.0 & 17.2 & 9.2 & 7.7 \\
\hline US & 80.7 & 75.5 & 20.2 & 17.5 & 9.9 & 8.4 \\
\hline NZ rank & 3 & 3 & 3 & 3 & 4 & 4 \\
\hline $\begin{array}{l}\text { Differenc } \\
\text { country, } y\end{array}$ & $\begin{array}{l}\text { to life exp } \\
\text { ears }\end{array}$ & ectanc & in New Z & ealand & & \\
\hline & At birth & & At age 65 & & At age 80 & \\
\hline 1961 & Females & Males & Females & Males & Females & Males \\
\hline Australia & 0.8 & -0.4 & 0.4 & -0.2 & 0.6 & 0.3 \\
\hline Canada & 0.7 & 0.0 & 0.8 & 0.8 & 0.9 & 0.8 \\
\hline UK & -0.1 & -0.6 & -0.4 & -0.9 & -0.1 & -0.3 \\
\hline US & 0.0 & -1.4 & 0.7 & 0.3 & 0.8 & 0.6 \\
\hline 2006 & & & & & & \\
\hline Australia & 1.6 & 1.1 & 1.1 & 0.6 & 0.5 & 0.3 \\
\hline Canada & 0.8 & 0.3 & 0.6 & 0.2 & 0.7 & 0.3 \\
\hline UK & -0.6 & -0.8 & -0.6 & -0.7 & -0.3 & -0.3 \\
\hline US & -1.5 & -2.5 & -0.4 & -0.5 & 0.4 & 0.4 \\
\hline
\end{tabular}


Table 4.2: New Zealand period life expectancy at birth, at age 65 and at age 80 compared to selected countries, 1961 and 2006, continued

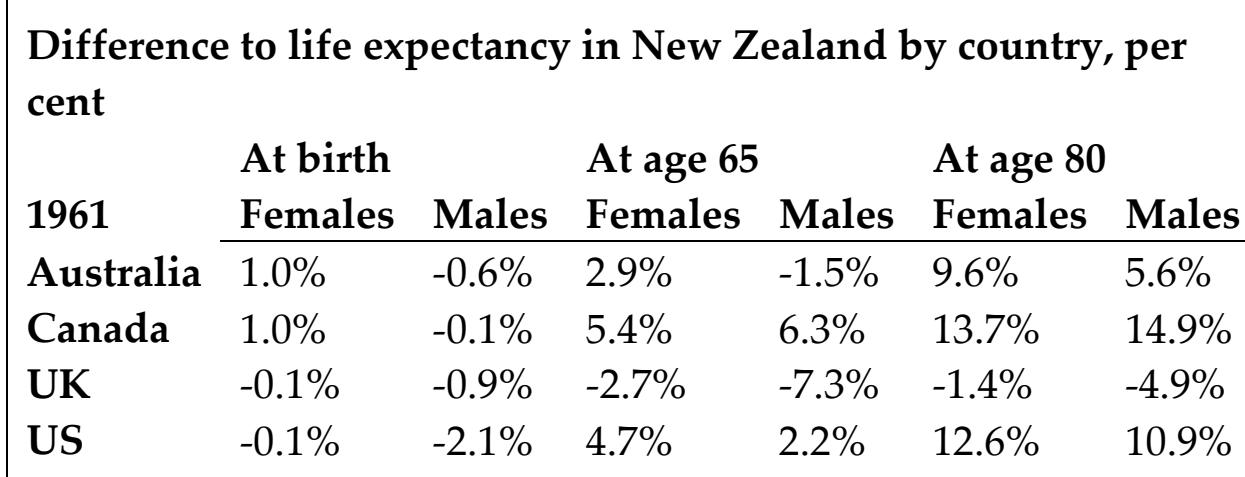

2006

$\begin{array}{lllllll}\text { Australia } & 2.0 \% & 1.4 \% & 5.1 \% & 3.5 \% & 5.4 \% & 3.9 \%\end{array}$

$\begin{array}{lllllll}\text { Canada } & 0.9 \% & 0.3 \% & 3.1 \% & 1.1 \% & 7.2 \% & 4.1 \%\end{array}$

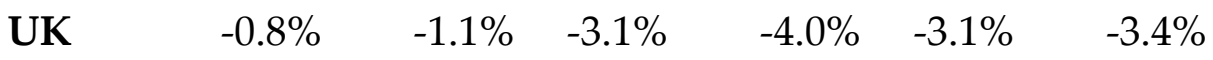

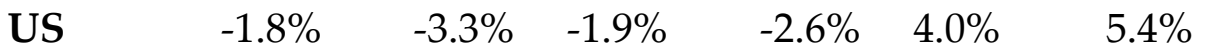

Source: New Zealand data from Statistics New Zealand life tables. Other mortality rates from Human Mortality Database life tables (downloaded August 2010).

The magnitude of the gap between New Zealand's life expectancy at birth and that of other countries in 2006 is consistent with what was found from the mortality rate analysis in Table 4.1: a small gap to the better measure in Canada, and a larger gap to the even better measure in Australia; and small and large gaps to the UK and US in the other direction. The gaps are roughly symmetrical for males, that is, New Zealand all ages mortality is as better compared to the UK as it is worse compared to Canada, and similarly for the US and Australia. For females, New Zealand's relative position is better, so all ages mortality is closer to the higher ranking Australia and Canada than to the laggards of the UK and US. 
This shape of mortality position changes at higher ages, as seen by the size of the difference between New Zealand's and other countries' life expectancy at ages 65 and 80 . The difference widens for all countries, with the US difference changing signs, as noted above. This is consistent with Table 4.1 as while life expectancy at age 65 is calculated with reference only to mortality rates from age 65 , life expectancy at birth is calculated from mortality rates at all ages, but gives less weight to mortality rates at ages 65 and over. Table 4.1 therefore gives a better comparison of the absolute level of mortality rates in age bands.

White (2002) showed that over the period 1955-1995, the period life expectancy at birth for 21 high income countries (including the five selected here) converged; that is that the average difference from the average life expectancy at birth was lower in 1995 than in 1955. Moreover, he showed that the change in life expectancy at birth over the period for any country was partially predicted by that country's level of life expectancy relative to the group mean. These conclusions do not hold for the smaller group selected here, over the later and slightly longer time period than White's. The average deviation from the group mean life expectancy at birth is shown in Table 4.3. It has increased over the period, although a large part of the increase can be attributed to the worsening relative position of the US. 
Table 4.3: Average absolute deviation from average life expectancy at birth for the selected countries, 1961 and 2006, in years

\begin{tabular}{|lccccc|}
\hline & \multicolumn{2}{c}{ Including US } & & \multicolumn{2}{c|}{ Excluding US } \\
\cline { 2 - 3 } \cline { 5 - 6 } & Females & Males & & Females & Males \\
\cline { 2 - 3 } $\mathbf{1 9 6 1}$ & 0.4 & 0.4 & & 0.4 & 0.3 \\
2006 & 0.9 & 1.0 & & 0.8 & 0.6 \\
\hline
\end{tabular}

Source: Calculated from life table data following White (2002) Table 3 for the UK and British settler countries selected here. New Zealand data from Statistics New Zealand life tables. Other mortality rates from Human Mortality Database life tables (downloaded August 2010).

White's conclusions suggest that the US would catch up with the group, having started from a spell of lower than average life expectancy. However, the US appears to be a special case. Not only has the gap between US and other countries' life expectancy at birth widened in recent decades, but the international ranking of US life expectancy crosses over from unfavourable to favourable from age 65 to age 80 . US demographic literature recognises data quality issues for the highest ages (Crimmins et al. 2010b). Further, many researchers have pointed out that the US has a health problem which is not a result purely of a difference in health provision or insurance, despite issues with those (see review in Banks et al. 2010 p. S220). The health problem is characterised as one of Americans becoming sicker at younger ages than Europeans. It is thought that more persistent higher levels of smoking behaviour in the US, especially higher smoking prevalence in lower socioeconomic groups, plays a large part. Contributions from other health causes, such as obesity, are possible but do not as yet have a firm evidence base (Crimmins et al. 2010b). Moreover, the US is thought to be exceptional in the extent to which income inequality is consistently negatively associated with population health. A review of nearly 100 epidemiological studies found that for other rich countries including Australia, Canada and New Zealand there 
was little or no effect on aggregate health from income inequality, with evidence on the UK inconclusive (Lynch et al. 2004). The mortality indicators in this study are consistent with the position of the US as a special case and therefore the US appears not to provide a plausible role model for New Zealand's mortality.

\subsection{Rate of mortality improvement}

In this section, the annual rates of improvement in mortality rates, QXI, are examined to give more detail on how New Zealand changed its relative mortality position over the last forty-five years. The results of the analysis are shown at an overall all ages level by country, then the following section shows more detail by age. Table 4.4 summarises to show average annual QXI in each past decade and over the whole period. Again, age standardisation is used so that the all ages figures from different countries and time periods can be compared without any effect from different age structures. 'All-ages' here refers to all ages up to and including age 90 .

The five selected countries have had different QXI over the forty-five period but each has been positive. Mortality has had a long-term trend of improvement. Tables in the next section show that some mortality rates worsened for a few five year age bands in the 1960s (all countries) and in the 1980s (all except Canada, and females in New Zealand and the UK). However, these mortality rate increases were limited in size and to a small number of age bands, so that the overall population trend was a reduction in mortality rates over each decade with one exception: males in the US in the 1960s. 
For all countries the 1960s were a poor decade with the all ages QXI being less than 1 per cent per annum for both males and females in all the five countries except UK females. The general picture is of constant improvement, but at a slower pace in the first decade of this analysis, that is, a strong period effect in the 1960s.

Table 4.4: Average age-standardised annual improvement in mortality (QXI) rates over decades and since 1961, all ages to age 90

\begin{tabular}{|lllllll|}
\hline & $\mathbf{1 9 6 1 -}$ & $\mathbf{1 9 7 1 -}$ & $\mathbf{1 9 8 1 -}$ & $\mathbf{1 9 9 1 -}$ & $\mathbf{1 9 9 6 -}$ & $\mathbf{1 9 6 1 -}$ \\
& $\mathbf{7 1}$ & $\mathbf{8 1}$ & $\mathbf{9 1}$ & $\mathbf{2 0 0 1}$ & $\mathbf{2 0 0 6}$ & $\mathbf{2 0 0 6}$ \\
\cline { 2 - 7 } Males & & & & & & \\
Australia & $0.2 \%$ & $2.6 \%$ & $2.6 \%$ & $2.5 \%$ & $3.6 \%$ & $2.2 \%$ \\
Canada & $0.4 \%$ & $2.1 \%$ & $2.7 \%$ & $2.8 \%$ & $2.5 \%$ & $2.0 \%$ \\
New Zealand & $0.0 \%$ & $1.6 \%$ & $1.3 \%$ & $3.3 \%$ & $2.9 \%$ & $1.7 \%$ \\
UK & $0.9 \%$ & $1.8 \%$ & $1.5 \%$ & $2.2 \%$ & $1.9 \%$ & $1.6 \%$ \\
US & $-0.3 \%$ & $2.3 \%$ & $1.0 \%$ & $2.4 \%$ & $1.9 \%$ & $1.3 \%$ \\
& & & & & & \\
Females & & & & & & \\
Australia & $0.3 \%$ & $3.5 \%$ & $2.0 \%$ & $2.5 \%$ & $2.6 \%$ & $2.1 \%$ \\
Canada & $0.6 \%$ & $2.5 \%$ & $2.3 \%$ & $1.7 \%$ & $1.9 \%$ & $1.8 \%$ \\
New Zealand & $0.4 \%$ & $1.5 \%$ & $2.0 \%$ & $2.6 \%$ & $2.7 \%$ & $1.7 \%$ \\
UK & $1.1 \%$ & $1.8 \%$ & $1.7 \%$ & $1.8 \%$ & $1.7 \%$ & $1.6 \%$ \\
US & $0.3 \%$ & $2.7 \%$ & $1.1 \%$ & $1.1 \%$ & $1.2 \%$ & $1.3 \%$ \\
\hline
\end{tabular}

Source: Calculated from mortality rates $\mathrm{q}_{\mathrm{x}}$. New Zealand mortality rates and population from Statistics New Zealand. Other mortality rates from Human Mortality Database life tables (downloaded August 2010).

Notes: The last decade shown 1996-2006 begins in the middle of the previous decade, 1991-2006. Each country's mortality rates at each time period are agestandardised to the New Zealand Estimated Resident Population of June 2006. To explore whether the choice of standard population would affect the results, an alternative standardisation to the UK population made (details not shown). The resulting QXI by decade do not change by more than one decimal place, and the interpretations below based on relative rankings do not change. 
Mortality improvement has been at the same pace for males and females within each country over the entire period except for Australia and Canada where improvement in male mortality has been faster. The ranking of QXI by country is the same for males and females. Australia has seen the fastest improvement with QXI over 2 per cent per annum for both males (2.2 per cent) and females (2.1 per cent) over the forty-five year period. Canada, New Zealand and the UK are close, with QXIs over the period being in the range 1.6 per cent to 1.8 per cent except Canadian males appear to have done better with QXI of 2 per cent. Mortality improvement has lagged in the US with QXI of 1.3 per cent for both males and females. The results of the previous section can now be put in some context. The change in the relative position of New Zealand's mortality was caused by relatively faster mortality improvement in Australia, and to a lesser extent in Canada, and slower mortality improvement in the US. New Zealand's average mortality improvement over the last fortyfive years ranks in the middle for this group of countries, just ahead in absolute terms of that for the UK.

A more complex picture emerges decade by decade. In the 1960s, New Zealand's QXI was poor, as was that in Australia and the US. In the 1970s, QXI increased for all countries, but New Zealand's was the worst for both males and females at 1.5 per cent (female) and 1.6 per cent (male). In the next decade, QXI for male New Zealanders decreased, but that for females increased to rank equal second to Australia. In the 1990s, QXI in New Zealand was the highest of all the selected countries at 2.6 per cent (female) and 3.3 per cent (male). This momentum carried on into the beginning of the next decade, with the New Zealand QXI for females in 1996-2006 being the highest at 2.7 
per cent and the male QXI at 2.5 per cent only second to an outperformance in Australia.

The QXI measure for New Zealand has gained momentum throughout the forty-five year period, with female mortality improvement accelerating in the 1980s before that of males in the 1990s. Australia had a poor decade in the 1960s, but has had relatively fast and constant improvement thereafter. In Canada, the shape of QXI for males is similar to that in Australia, but for females there has been a slower pace of improvement since the 1990s. QXI in the UK has been relatively constant after the 1960s, although the 1990s were better for males than females. The US has not shown a consistent pattern.

\subsection{Mortality improvement by age and cohort}

Further insights can be seen from examining QXI in more detail by age. QXI by five year age bands are summarised for the entire period in Figure 4.7 and Figure 4.8. The average rates of mortality improvement over the forty-five year period follow a similar pattern by age in each country. QXI are highest (around 3 to 3.5 per cent per annum for both sexes) at the youngest ages then fall to a low at ages 20 to 30 for males and ages 15 to 20 for females. QXI for males climb to a higher level centred on 2 per cent per annum around age 60 before falling back to 1 per cent at the oldest ages. For females, QXI are flatter from age 30. The countries with highest and lowest QXI overall, Australia and the US, generally achieve the highest and lowest QXI at most ages for both males and females. The remaining three countries - Canada, the UK and New Zealand - take different rankings at different ages. The relatively greater improvement in New Zealand after age 40, lagging only Australia, can be seen for both males and females. 
Figure 4.7: Average age-standardised annual improvement in mortality rates (QXI), 1961-2006, all ages to age 90, in five year age bands, males

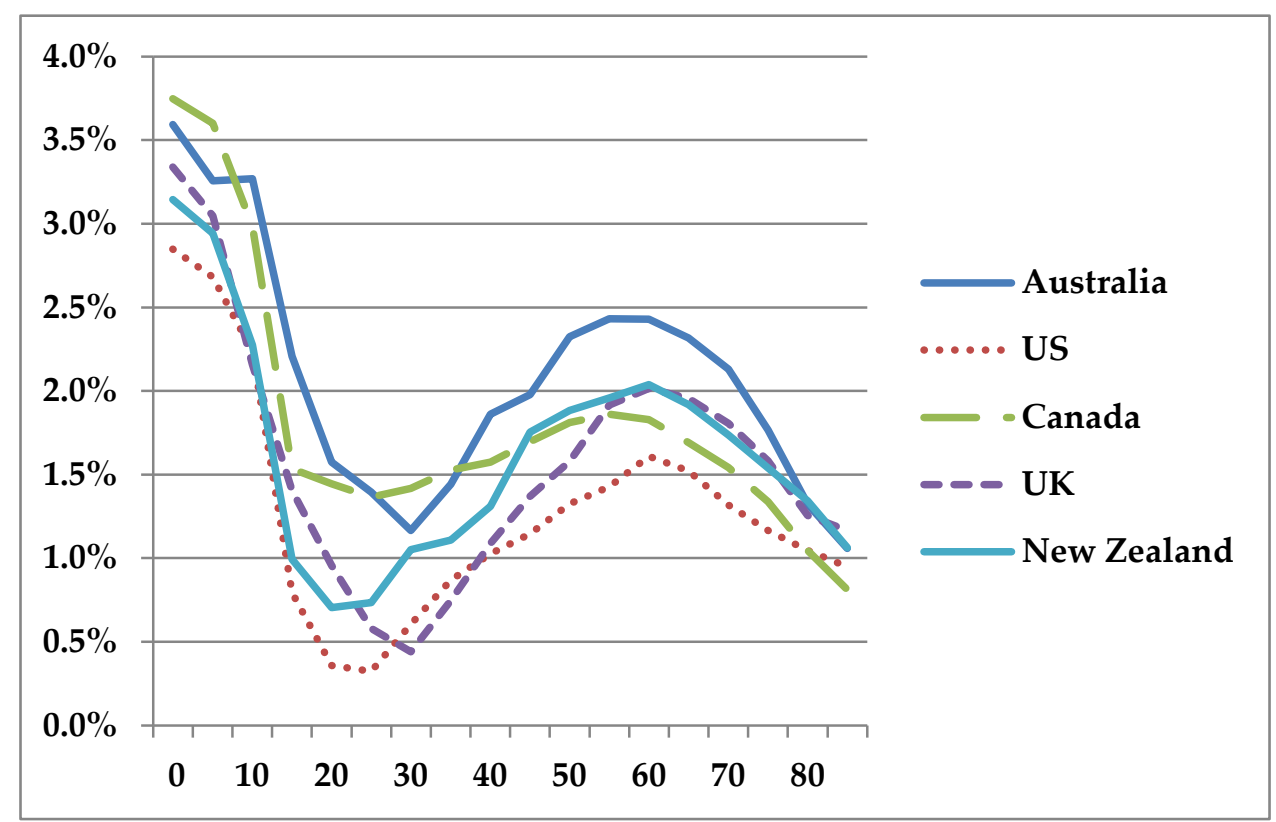

Source: See Table 4.4.

Figure 4.8: Average age-standardised annual improvement in mortality rates (QXI), 1961-2006, all ages to age 90, in five year age bands, females

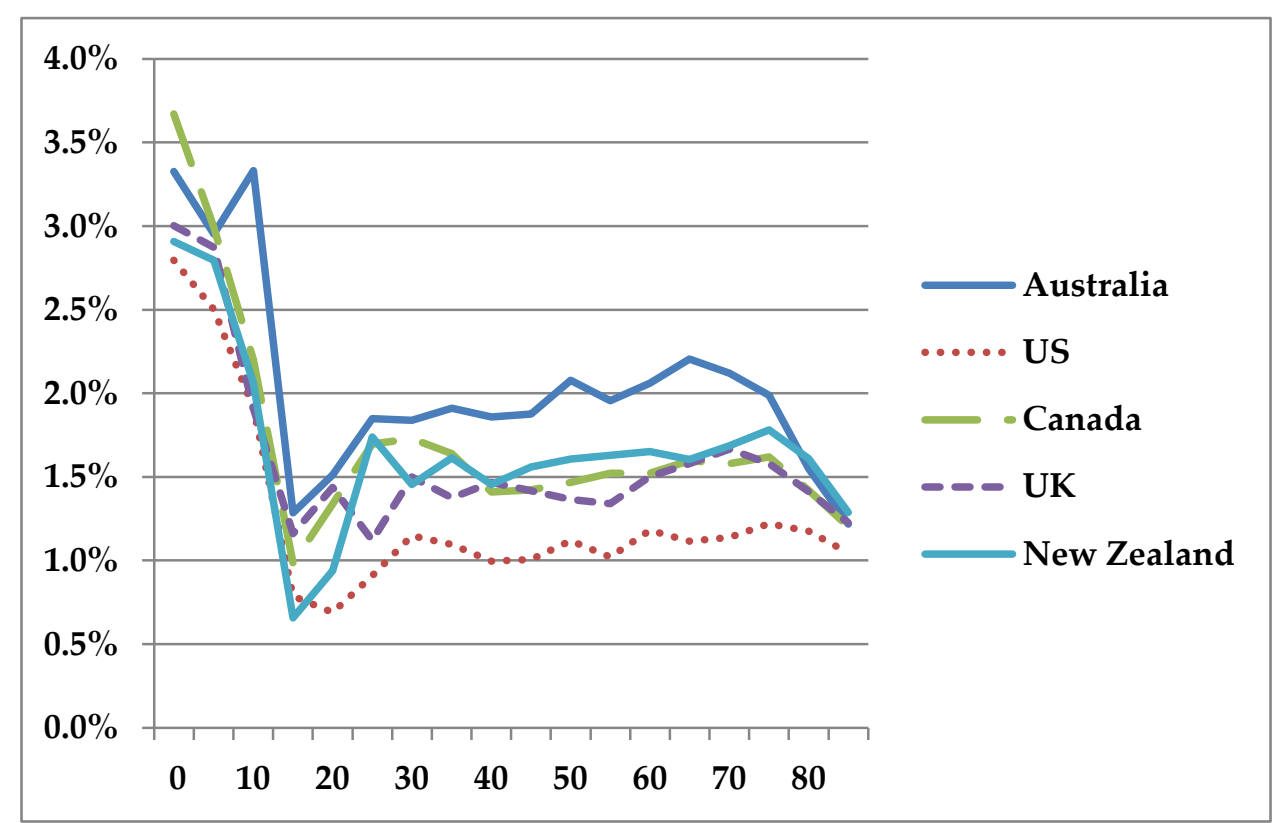

Source: See Table 4.4 . 
Now looking within the whole period, QXI from each country by five year age bands for each decade are shown in Table 4.5. The table shows further information as cells are highlighted where the value is above the average for ages 20 and over in that period plus one standard deviation. These cells show the age bands at which mortality rates improved most rapidly in that period, relative to the other five-year gender-specific adult age bands for that country. These are referred to as significant QXI. A lack of highlighting in any column indicates mortality improvement was evenly spread across adult ages. Ages under 20 were not included in the range because rates of improvement have been high at these ages in most periods and most countries, and the focus of this analysis is to identify any cohort trends at adult ages. This analysis uses the methodology of that in O'Connell and Dunstan (2009), as described in section 3.4. 
Table 4.5: Average age-standardised annual improvement in mortality rates (QXI) by country and decade for five year age bands starting at age shown

\begin{tabular}{|c|c|c|c|c|c|c|}
\hline \multicolumn{7}{|c|}{ Australia, males } \\
\hline Age & $\begin{array}{l}\text { 1961- } \\
71\end{array}$ & $\begin{array}{l}1971- \\
81\end{array}$ & $\begin{array}{l}\text { 1981- } \\
91\end{array}$ & $\begin{array}{l}1991- \\
2001\end{array}$ & $\begin{array}{l}1996- \\
2006\end{array}$ & $\begin{array}{l}1961- \\
2006\end{array}$ \\
\hline 0 & $1.8 \%$ & $4.2 \%$ & $5.0 \%$ & $2.9 \%$ & $5.1 \%$ & $3.6 \%$ \\
\hline 5 & $0.6 \%$ & $3.0 \%$ & $5.3 \%$ & $3.4 \%$ & $4.2 \%$ & $3.3 \%$ \\
\hline 10 & $1.5 \%$ & $4.4 \%$ & $3.1 \%$ & $2.8 \%$ & $5.5 \%$ & $3.3 \%$ \\
\hline 15 & $-1.7 \%$ & $2.7 \%$ & $2.9 \%$ & $2.8 \%$ & $5.0 \%$ & $2.2 \%$ \\
\hline 20 & $-1.4 \%$ & $2.0 \%$ & $1.7 \%$ & $2.4 \%$ & $4.3 \%$ & $1.6 \%$ \\
\hline 25 & $0.3 \%$ & $0.6 \%$ & $0.5 \%$ & $1.5 \%$ & $4.5 \%$ & $1.4 \%$ \\
\hline 30 & $0.9 \%$ & $2.1 \%$ & $-0.7 \%$ & $0.9 \%$ & $3.4 \%$ & $1.2 \%$ \\
\hline 35 & $0.1 \%$ & $3.2 \%$ & $0.2 \%$ & $1.6 \%$ & $2.6 \%$ & $1.4 \%$ \\
\hline 40 & $1.4 \%$ & $2.3 \%$ & $2.7 \%$ & $1.4 \%$ & $1.8 \%$ & $1.9 \%$ \\
\hline 45 & $-0.1 \%$ & $2.8 \%$ & $3.6 \%$ & $2.2 \%$ & $1.2 \%$ & $2.0 \%$ \\
\hline 50 & $0.1 \%$ & $2.2 \%$ & $4.1 \%$ & $3.5 \%$ & $2.4 \%$ & $2.3 \%$ \\
\hline 55 & $-0.2 \%$ & $2.4 \%$ & $3.8 \%$ & $3.2 \%$ & $3.0 \%$ & $2.4 \%$ \\
\hline 60 & $0.1 \%$ & $2.6 \%$ & $2.5 \%$ & $3.8 \%$ & $4.0 \%$ & $2.4 \%$ \\
\hline 65 & $0.0 \%$ & $2.3 \%$ & $2.5 \%$ & $3.7 \%$ & $4.1 \%$ & $2.3 \%$ \\
\hline 70 & $-0.1 \%$ & $1.9 \%$ & $2.7 \%$ & $3.0 \%$ & $4.2 \%$ & $2.1 \%$ \\
\hline 75 & $-0.4 \%$ & $1.9 \%$ & $2.0 \%$ & $2.8 \%$ & $3.4 \%$ & $1.8 \%$ \\
\hline 80 & $-0.2 \%$ & $1.3 \%$ & $1.3 \%$ & $2.5 \%$ & $3.0 \%$ & $1.3 \%$ \\
\hline 85 & $-0.4 \%$ & $1.6 \%$ & $1.3 \%$ & $0.9 \%$ & $2.5 \%$ & $1.1 \%$ \\
\hline Average & $0.2 \%$ & $2.6 \%$ & $2.6 \%$ & $2.5 \%$ & $3.6 \%$ & $2.2 \%$ \\
\hline
\end{tabular}

Source: See Table 4.4.

Notes: Average shown is standard weighted average, all ages 0 to 90 .

Standard weights for five-year age bands and all ages average from New Zealand Estimated Resident Population 2006.

Cells with bold figures in a box are those with a value higher than the simple average plus one standard deviation of the values for that time period for ages 20 and over. The calculation is carried out using unrounded QXI values. QXI values are rounded to one decimal place in the table.

Table continues on following pages. 
Table 4.5: Average age-standardised annual improvement in mortality rates (QXI) by country and decade for five year age bands starting at age shown, continued

\begin{tabular}{|c|c|c|c|c|c|c|}
\hline \multicolumn{7}{|c|}{ Australia, females } \\
\hline Age & $\begin{array}{l}\text { 1961- } \\
71\end{array}$ & $\begin{array}{l}1971- \\
81\end{array}$ & $\begin{array}{l}\text { 1981- } \\
91\end{array}$ & $\begin{array}{l}1991- \\
2001\end{array}$ & $\begin{array}{l}1996- \\
2006\end{array}$ & $\begin{array}{l}1961- \\
2006\end{array}$ \\
\hline 0 & $2.0 \%$ & $5.1 \%$ & $4.4 \%$ & $2.8 \%$ & $3.3 \%$ & $3.3 \%$ \\
\hline 5 & $1.8 \%$ & $5.2 \%$ & $2.9 \%$ & $3.5 \%$ & $1.6 \%$ & $3.0 \%$ \\
\hline 10 & $3.5 \%$ & $0.9 \%$ & $3.5 \%$ & $3.1 \%$ & $8.8 \%$ & $3.3 \%$ \\
\hline 15 & $-3.6 \%$ & $4.2 \%$ & $2.1 \%$ & $3.7 \%$ & $0.8 \%$ & $1.3 \%$ \\
\hline 20 & $-0.4 \%$ & $2.8 \%$ & $0.6 \%$ & $2.5 \%$ & $0.7 \%$ & $1.5 \%$ \\
\hline 25 & $1.3 \%$ & $2.4 \%$ & $-0.4 \%$ & $3.8 \%$ & $2.6 \%$ & $1.8 \%$ \\
\hline 30 & $-0.1 \%$ & $4.7 \%$ & $0.5 \%$ & $1.3 \%$ & $2.3 \%$ & $1.8 \%$ \\
\hline 35 & $0.9 \%$ & $4.2 \%$ & $1.2 \%$ & $1.0 \%$ & $2.2 \%$ & $1.9 \%$ \\
\hline 40 & $-0.6 \%$ & $4.4 \%$ & $2.4 \%$ & $0.5 \%$ & $1.5 \%$ & $1.9 \%$ \\
\hline 45 & $-0.7 \%$ & $3.4 \%$ & $3.3 \%$ & $2.2 \%$ & $1.1 \%$ & $1.9 \%$ \\
\hline 50 & $0.0 \%$ & $3.6 \%$ & $2.0 \%$ & $2.5 \%$ & $2.6 \%$ & $2.1 \%$ \\
\hline 55 & $-0.6 \%$ & $3.0 \%$ & $2.4 \%$ & $2.3 \%$ & $3.1 \%$ & $2.0 \%$ \\
\hline 60 & $0.1 \%$ & $2.7 \%$ & $2.0 \%$ & $3.3 \%$ & $3.2 \%$ & $2.1 \%$ \\
\hline 65 & $0.8 \%$ & $2.5 \%$ & $1.7 \%$ & $3.2 \%$ & $3.5 \%$ & $2.2 \%$ \\
\hline 70 & $0.2 \%$ & $3.2 \%$ & $1.5 \%$ & $2.7 \%$ & $3.3 \%$ & $2.1 \%$ \\
\hline 75 & $0.2 \%$ & $3.2 \%$ & $1.5 \%$ & $2.8 \%$ & $3.2 \%$ & $2.0 \%$ \\
\hline 80 & $0.2 \%$ & $2.3 \%$ & $1.6 \%$ & $1.9 \%$ & $2.3 \%$ & $1.5 \%$ \\
\hline 85 & $-0.1 \%$ & $2.1 \%$ & $1.5 \%$ & $1.1 \%$ & $2.0 \%$ & $1.2 \%$ \\
\hline Average & $0.3 \%$ & $3.5 \%$ & $2.0 \%$ & $2.5 \%$ & $2.6 \%$ & $2.1 \%$ \\
\hline
\end{tabular}

For notes see page 110 . 
Table 4.5: Average age-standardised annual improvement in mortality rates (QXI) by country and decade for five year age bands starting at age shown, continued

\begin{tabular}{|c|c|c|c|c|c|c|}
\hline \multicolumn{7}{|c|}{ Canada, males } \\
\hline Age & $\begin{array}{l}\text { 1961- } \\
71\end{array}$ & $\begin{array}{l}\text { 1971- } \\
81\end{array}$ & $\begin{array}{l}\text { 1981- } \\
91\end{array}$ & $\begin{array}{l}1991- \\
2001\end{array}$ & $\begin{array}{l}1996- \\
2006\end{array}$ & $\begin{array}{l}1961- \\
2006\end{array}$ \\
\hline 0 & $3.4 \%$ & $4.6 \%$ & $4.4 \%$ & $3.0 \%$ & $3.0 \%$ & $3.7 \%$ \\
\hline 5 & $1.0 \%$ & $5.2 \%$ & $5.1 \%$ & $4.1 \%$ & $2.3 \%$ & $3.6 \%$ \\
\hline 10 & $1.2 \%$ & $2.4 \%$ & $4.3 \%$ & $5.3 \%$ & $2.7 \%$ & $3.0 \%$ \\
\hline 15 & $-1.6 \%$ & $0.7 \%$ & $3.4 \%$ & $2.8 \%$ & $1.6 \%$ & $1.5 \%$ \\
\hline 20 & $-0.6 \%$ & $1.2 \%$ & $2.6 \%$ & $2.9 \%$ & $1.8 \%$ & $1.4 \%$ \\
\hline 25 & $0.4 \%$ & $0.8 \%$ & $0.9 \%$ & $3.4 \%$ & $2.6 \%$ & $1.4 \%$ \\
\hline 30 & $0.1 \%$ & $1.6 \%$ & $0.4 \%$ & $2.9 \%$ & $3.9 \%$ & $1.4 \%$ \\
\hline 35 & $0.6 \%$ & $2.0 \%$ & $1.0 \%$ & $2.3 \%$ & $3.5 \%$ & $1.5 \%$ \\
\hline 40 & $-0.4 \%$ & $2.5 \%$ & $2.5 \%$ & $1.8 \%$ & $2.6 \%$ & $1.6 \%$ \\
\hline 45 & $0.1 \%$ & $2.0 \%$ & $3.0 \%$ & $1.9 \%$ & $1.3 \%$ & $1.7 \%$ \\
\hline 50 & $0.4 \%$ & $1.9 \%$ & $3.1 \%$ & $2.0 \%$ & $1.8 \%$ & $1.8 \%$ \\
\hline 55 & $0.5 \%$ & $1.6 \%$ & $2.8 \%$ & $2.6 \%$ & $2.3 \%$ & $1.9 \%$ \\
\hline 60 & $0.6 \%$ & $1.5 \%$ & $2.2 \%$ & $2.5 \%$ & $2.6 \%$ & $1.8 \%$ \\
\hline 65 & $0.3 \%$ & $1.2 \%$ & $1.9 \%$ & $2.7 \%$ & $3.2 \%$ & $1.7 \%$ \\
\hline 70 & $0.4 \%$ & $1.2 \%$ & $1.6 \%$ & $2.1 \%$ & $3.4 \%$ & $1.5 \%$ \\
\hline 75 & $0.4 \%$ & $0.9 \%$ & $1.2 \%$ & $2.0 \%$ & $2.7 \%$ & $1.3 \%$ \\
\hline 80 & $0.4 \%$ & $0.6 \%$ & $0.7 \%$ & $1.6 \%$ & $2.6 \%$ & $1.0 \%$ \\
\hline 85 & $0.3 \%$ & $0.9 \%$ & $0.2 \%$ & $1.1 \%$ & $2.1 \%$ & $0.8 \%$ \\
\hline Average & $0.4 \%$ & $2.1 \%$ & $2.7 \%$ & $2.8 \%$ & $2.5 \%$ & $2.0 \%$ \\
\hline
\end{tabular}

For notes see page 110 . 
Table 4.5: Average age-standardised annual improvement in mortality rates (QXI) by country and decade for five year age bands starting at age shown, continued

\begin{tabular}{|c|c|c|c|c|c|c|}
\hline \multicolumn{7}{|c|}{ Canada, females } \\
\hline Age & $\begin{array}{l}\text { 1961- } \\
71\end{array}$ & $\begin{array}{l}1971- \\
81\end{array}$ & $\begin{array}{l}\text { 1981- } \\
91\end{array}$ & $\begin{array}{l}1991- \\
2001\end{array}$ & $\begin{array}{l}1996- \\
2006\end{array}$ & $\begin{array}{l}1961- \\
2006\end{array}$ \\
\hline 0 & $2.5 \%$ & $5.2 \%$ & $5.1 \%$ & $2.1 \%$ & $3.5 \%$ & $3.7 \%$ \\
\hline 5 & $0.0 \%$ & $4.4 \%$ & $4.2 \%$ & $2.9 \%$ & $3.3 \%$ & $3.0 \%$ \\
\hline 10 & $-0.8 \%$ & $4.0 \%$ & $2.7 \%$ & $3.1 \%$ & $2.1 \%$ & $2.2 \%$ \\
\hline 15 & $-1.7 \%$ & $1.8 \%$ & $2.1 \%$ & $1.9 \%$ & $0.9 \%$ & $1.0 \%$ \\
\hline 20 & $-0.2 \%$ & $2.7 \%$ & $2.2 \%$ & $1.3 \%$ & $0.5 \%$ & $1.3 \%$ \\
\hline 25 & $1.5 \%$ & $1.0 \%$ & $1.9 \%$ & $2.6 \%$ & $1.3 \%$ & $1.7 \%$ \\
\hline 30 & $0.3 \%$ & $3.1 \%$ & $1.6 \%$ & $1.4 \%$ & $2.6 \%$ & $1.7 \%$ \\
\hline 35 & $0.6 \%$ & $3.0 \%$ & $1.7 \%$ & $0.7 \%$ & $1.8 \%$ & $1.6 \%$ \\
\hline 40 & $-0.4 \%$ & $2.3 \%$ & $2.4 \%$ & $1.3 \%$ & $2.2 \%$ & $1.4 \%$ \\
\hline 45 & $0.6 \%$ & $1.7 \%$ & $2.5 \%$ & $1.1 \%$ & $1.2 \%$ & $1.4 \%$ \\
\hline 50 & $1.4 \%$ & $1.2 \%$ & $2.4 \%$ & $1.5 \%$ & $1.1 \%$ & $1.5 \%$ \\
\hline 55 & $1.0 \%$ & $1.4 \%$ & $1.8 \%$ & $1.8 \%$ & $2.3 \%$ & $1.5 \%$ \\
\hline 60 & $1.6 \%$ & $1.2 \%$ & $1.7 \%$ & $1.7 \%$ & $1.9 \%$ & $1.5 \%$ \\
\hline 65 & $2.1 \%$ & $1.2 \%$ & $1.4 \%$ & $1.3 \%$ & $1.9 \%$ & $1.6 \%$ \\
\hline 70 & $1.9 \%$ & $1.7 \%$ & $1.3 \%$ & $1.2 \%$ & $1.9 \%$ & $1.6 \%$ \\
\hline 75 & $2.1 \%$ & $2.0 \%$ & $0.9 \%$ & $1.4 \%$ & $2.0 \%$ & $1.6 \%$ \\
\hline 80 & $1.5 \%$ & $1.7 \%$ & $1.0 \%$ & $1.1 \%$ & $2.1 \%$ & $1.4 \%$ \\
\hline 85 & $1.2 \%$ & $2.0 \%$ & $0.6 \%$ & $0.6 \%$ & $1.6 \%$ & $1.2 \%$ \\
\hline Average & $0.6 \%$ & $2.5 \%$ & $2.3 \%$ & $1.7 \%$ & $1.9 \%$ & $1.8 \%$ \\
\hline
\end{tabular}

For notes see page 110 . 
Table 4.5: Average age-standardised annual improvement in mortality rates (QXI) by country and decade for five year age bands starting at age shown, continued

\begin{tabular}{|c|c|c|c|c|c|c|}
\hline \multicolumn{7}{|c|}{ New Zealand, males } \\
\hline Age & $\begin{array}{c}1961- \\
71\end{array}$ & $\begin{array}{c}1971- \\
81\end{array}$ & $\begin{array}{c}1981- \\
91\end{array}$ & $\begin{array}{l}1991- \\
2001\end{array}$ & $\begin{array}{c}1996- \\
2006\end{array}$ & $\begin{array}{l}1961- \\
2006\end{array}$ \\
\hline 0 & $2.4 \%$ & $2.9 \%$ & $2.6 \%$ & $4.6 \%$ & $3.2 \%$ & $3.1 \%$ \\
\hline 5 & $1.6 \%$ & $2.7 \%$ & $2.7 \%$ & $5.1 \%$ & $3.7 \%$ & $2.9 \%$ \\
\hline 10 & $1.5 \%$ & $3.4 \%$ & $-0.1 \%$ & $3.3 \%$ & $2.2 \%$ & $2.3 \%$ \\
\hline 15 & $-2.7 \%$ & $1.2 \%$ & $0.3 \%$ & $3.5 \%$ & $4.4 \%$ & $1.0 \%$ \\
\hline 20 & $-1.0 \%$ & $-0.8 \%$ & $-0.9 \%$ & $5.0 \%$ & $3.7 \%$ & $0.7 \%$ \\
\hline 25 & $0.2 \%$ & $-0.9 \%$ & $-0.3 \%$ & $2.3 \%$ & $3.5 \%$ & $0.7 \%$ \\
\hline 30 & $0.2 \%$ & $1.1 \%$ & $0.0 \%$ & $1.2 \%$ & $3.0 \%$ & $1.1 \%$ \\
\hline 35 & $-0.4 \%$ & $2.4 \%$ & $-0.1 \%$ & $2.4 \%$ & $1.0 \%$ & $1.1 \%$ \\
\hline 40 & $-0.6 \%$ & $2.1 \%$ & $2.2 \%$ & $1.9 \%$ & $0.6 \%$ & $1.3 \%$ \\
\hline 45 & $0.0 \%$ & $1.5 \%$ & $2.7 \%$ & $2.9 \%$ & $1.9 \%$ & $1.8 \%$ \\
\hline 50 & $-0.4 \%$ & $2.0 \%$ & $2.3 \%$ & $3.6 \%$ & $3.0 \%$ & $1.9 \%$ \\
\hline 55 & $-0.7 \%$ & $1.5 \%$ & $2.7 \%$ & $3.9 \%$ & $3.8 \%$ & $2.0 \%$ \\
\hline 60 & $-0.4 \%$ & $1.5 \%$ & $2.8 \%$ & $3.5 \%$ & $4.1 \%$ & $2.0 \%$ \\
\hline 65 & $-0.5 \%$ & $1.2 \%$ & $2.5 \%$ & $3.3 \%$ & $4.1 \%$ & $1.9 \%$ \\
\hline 70 & $-0.3 \%$ & $1.0 \%$ & $2.1 \%$ & $2.8 \%$ & $3.8 \%$ & $1.7 \%$ \\
\hline 75 & $-0.4 \%$ & $0.9 \%$ & $2.0 \%$ & $2.5 \%$ & $3.2 \%$ & $1.5 \%$ \\
\hline 80 & $-0.1 \%$ & $0.9 \%$ & $1.3 \%$ & $2.4 \%$ & $2.8 \%$ & $1.3 \%$ \\
\hline 85 & $0.2 \%$ & $0.8 \%$ & $1.8 \%$ & $1.0 \%$ & $1.8 \%$ & $1.1 \%$ \\
\hline Average & $0.0 \%$ & $1.6 \%$ & $1.3 \%$ & $3.3 \%$ & $2.9 \%$ & $1.7 \%$ \\
\hline
\end{tabular}

For notes see page 110 . 
Table 4.5: Average age-standardised annual improvement in mortality rates (QXI) by country and decade for five year age bands starting at age shown, continued

\begin{tabular}{|c|c|c|c|c|c|c|}
\hline \multicolumn{7}{|c|}{ New Zealand, females } \\
\hline Age & $\begin{array}{l}1961- \\
71\end{array}$ & $\begin{array}{l}1971- \\
81\end{array}$ & $\begin{array}{l}1981- \\
91\end{array}$ & $\begin{array}{l}1991- \\
2001\end{array}$ & $\begin{array}{l}1996- \\
2006\end{array}$ & $\begin{array}{l}1961- \\
2006\end{array}$ \\
\hline 0 & $2.3 \%$ & $2.5 \%$ & $5.3 \%$ & $1.6 \%$ & $2.2 \%$ & $2.9 \%$ \\
\hline 5 & $1.4 \%$ & $3.9 \%$ & $0.5 \%$ & $3.7 \%$ & $3.3 \%$ & $2.8 \%$ \\
\hline 10 & $0.9 \%$ & $2.6 \%$ & $3.3 \%$ & $0.9 \%$ & $3.7 \%$ & $2.1 \%$ \\
\hline 15 & $-1.5 \%$ & $-0.4 \%$ & $2.0 \%$ & $3.8 \%$ & $2.9 \%$ & $0.7 \%$ \\
\hline 20 & $-0.3 \%$ & $-1.3 \%$ & $1.8 \%$ & $2.8 \%$ & $4.1 \%$ & $0.9 \%$ \\
\hline 25 & $0.9 \%$ & $0.7 \%$ & $1.1 \%$ & $1.4 \%$ & $4.9 \%$ & $1.7 \%$ \\
\hline 30 & $0.7 \%$ & $2.0 \%$ & $1.1 \%$ & $2.0 \%$ & $1.5 \%$ & $1.5 \%$ \\
\hline 35 & $0.7 \%$ & $2.7 \%$ & $1.3 \%$ & $2.9 \%$ & $1.1 \%$ & $1.6 \%$ \\
\hline 40 & $-0.5 \%$ & $2.1 \%$ & $2.1 \%$ & $3.0 \%$ & $1.0 \%$ & $1.5 \%$ \\
\hline 45 & $-0.6 \%$ & $0.6 \%$ & $2.9 \%$ & $3.3 \%$ & $2.1 \%$ & $1.6 \%$ \\
\hline 50 & $-0.2 \%$ & $1.3 \%$ & $1.2 \%$ & $3.6 \%$ & $3.3 \%$ & $1.6 \%$ \\
\hline 55 & $0.4 \%$ & $0.6 \%$ & $1.8 \%$ & $2.8 \%$ & $3.5 \%$ & $1.6 \%$ \\
\hline 60 & $0.5 \%$ & $1.2 \%$ & $1.7 \%$ & $2.1 \%$ & $3.2 \%$ & $1.7 \%$ \\
\hline 65 & $0.3 \%$ & $0.7 \%$ & $2.4 \%$ & $2.2 \%$ & $2.9 \%$ & $1.6 \%$ \\
\hline 70 & $0.7 \%$ & $1.3 \%$ & $1.9 \%$ & $2.6 \%$ & $2.6 \%$ & $1.7 \%$ \\
\hline 75 & $0.9 \%$ & $1.9 \%$ & $1.9 \%$ & $2.1 \%$ & $2.5 \%$ & $1.8 \%$ \\
\hline 80 & $0.9 \%$ & $1.8 \%$ & $1.5 \%$ & $2.1 \%$ & $2.1 \%$ & $1.6 \%$ \\
\hline 85 & $0.4 \%$ & $2.4 \%$ & $1.3 \%$ & $1.3 \%$ & $1.5 \%$ & $1.3 \%$ \\
\hline Average & $0.4 \%$ & $1.5 \%$ & $2.0 \%$ & $2.6 \%$ & $2.7 \%$ & $1.7 \%$ \\
\hline
\end{tabular}

For notes see page 110 . 
Table 4.5: Average age-standardised annual improvement in mortality rates (QXI) by country and decade for five year age bands starting at age shown, continued

\begin{tabular}{|c|c|c|c|c|c|c|}
\hline \multicolumn{7}{|c|}{ UK, males } \\
\hline Age & $\begin{array}{l}1961- \\
71\end{array}$ & $\begin{array}{l}1971- \\
81\end{array}$ & $\begin{array}{l}1981- \\
91\end{array}$ & $\begin{array}{l}1991- \\
2001\end{array}$ & $\begin{array}{l}1996- \\
2006\end{array}$ & $\begin{array}{l}1961- \\
2006\end{array}$ \\
\hline 0 & $2.5 \%$ & $3.7 \%$ & $3.4 \%$ & $4.6 \%$ & $3.3 \%$ & $3.3 \%$ \\
\hline 5 & $1.1 \%$ & $4.6 \%$ & $2.6 \%$ & $5.7 \%$ & $1.0 \%$ & $3.0 \%$ \\
\hline 10 & $0.5 \%$ & $2.7 \%$ & $2.6 \%$ & $3.1 \%$ & $2.1 \%$ & $2.2 \%$ \\
\hline 15 & $-0.2 \%$ & $1.4 \%$ & $0.8 \%$ & $3.1 \%$ & $2.6 \%$ & $1.4 \%$ \\
\hline 20 & $1.2 \%$ & $1.1 \%$ & $-0.4 \%$ & $0.9 \%$ & $2.1 \%$ & $1.0 \%$ \\
\hline 25 & $1.8 \%$ & $0.2 \%$ & $-0.4 \%$ & $0.3 \%$ & $1.6 \%$ & $0.6 \%$ \\
\hline 30 & $1.5 \%$ & $1.1 \%$ & $-0.4 \%$ & $-0.8 \%$ & $0.6 \%$ & $0.4 \%$ \\
\hline 35 & $1.4 \%$ & $1.9 \%$ & $-0.4 \%$ & $0.5 \%$ & $0.2 \%$ & $0.7 \%$ \\
\hline 40 & $0.3 \%$ & $1.8 \%$ & $1.6 \%$ & $0.9 \%$ & $0.9 \%$ & $1.1 \%$ \\
\hline 45 & $0.0 \%$ & $1.9 \%$ & $2.5 \%$ & $1.0 \%$ & $1.1 \%$ & $1.4 \%$ \\
\hline 50 & $0.3 \%$ & $1.3 \%$ & $2.8 \%$ & $2.2 \%$ & $1.3 \%$ & $1.6 \%$ \\
\hline 55 & $1.1 \%$ & $0.8 \%$ & $3.1 \%$ & $2.6 \%$ & $2.5 \%$ & $1.9 \%$ \\
\hline 60 & $1.2 \%$ & $1.2 \%$ & $2.3 \%$ & $3.1 \%$ & $2.9 \%$ & $2.0 \%$ \\
\hline 65 & $0.7 \%$ & $1.4 \%$ & $1.6 \%$ & $3.5 \%$ & $3.6 \%$ & $2.0 \%$ \\
\hline 70 & $0.4 \%$ & $1.1 \%$ & $1.7 \%$ & $2.8 \%$ & $3.9 \%$ & $1.8 \%$ \\
\hline 75 & $0.8 \%$ & $0.6 \%$ & $1.6 \%$ & $2.2 \%$ & $3.1 \%$ & $1.6 \%$ \\
\hline 80 & $0.7 \%$ & $0.3 \%$ & $1.3 \%$ & $2.2 \%$ & $2.5 \%$ & $1.3 \%$ \\
\hline 85 & $1.1 \%$ & $0.3 \%$ & $1.0 \%$ & $1.5 \%$ & $2.4 \%$ & $1.2 \%$ \\
\hline Average & $0.9 \%$ & $1.8 \%$ & $1.5 \%$ & $2.2 \%$ & $1.9 \%$ & $1.6 \%$ \\
\hline
\end{tabular}

For notes see page 110 . 
Table 4.5: Average age-standardised annual improvement in mortality rates (QXI) by country and decade for five year age bands starting at age shown, continued

\begin{tabular}{|c|c|c|c|c|c|c|}
\hline \multicolumn{7}{|c|}{ UK, females } \\
\hline Age & $\begin{array}{l}\text { 1961- } \\
71\end{array}$ & $\begin{array}{l}1971- \\
81\end{array}$ & $\begin{array}{l}\text { 1981- } \\
91\end{array}$ & $\begin{array}{l}1991- \\
2001\end{array}$ & $\begin{array}{l}1996- \\
2006\end{array}$ & $\begin{array}{l}1961- \\
2006\end{array}$ \\
\hline 0 & $2.4 \%$ & $3.8 \%$ & $3.1 \%$ & $3.7 \%$ & $1.3 \%$ & $3.0 \%$ \\
\hline 5 & $1.1 \%$ & $3.6 \%$ & $2.1 \%$ & $3.2 \%$ & $2.4 \%$ & $2.9 \%$ \\
\hline 10 & $0.7 \%$ & $2.1 \%$ & $2.3 \%$ & $3.3 \%$ & $1.0 \%$ & $1.9 \%$ \\
\hline 15 & $0.0 \%$ & $1.7 \%$ & $0.9 \%$ & $1.8 \%$ & $2.4 \%$ & $1.2 \%$ \\
\hline 20 & $1.9 \%$ & $1.7 \%$ & $0.4 \%$ & $0.8 \%$ & $1.9 \%$ & $1.4 \%$ \\
\hline 25 & $1.6 \%$ & $1.3 \%$ & $1.5 \%$ & $0.9 \%$ & $1.0 \%$ & $1.1 \%$ \\
\hline 30 & $1.9 \%$ & $1.8 \%$ & $1.4 \%$ & $0.8 \%$ & $1.7 \%$ & $1.5 \%$ \\
\hline 35 & $1.6 \%$ & $2.2 \%$ & $1.1 \%$ & $1.0 \%$ & $1.2 \%$ & $1.4 \%$ \\
\hline 40 & $0.9 \%$ & $2.1 \%$ & $2.3 \%$ & $0.5 \%$ & $1.2 \%$ & $1.5 \%$ \\
\hline 45 & $0.2 \%$ & $2.0 \%$ & $2.4 \%$ & $1.2 \%$ & $1.1 \%$ & $1.4 \%$ \\
\hline 50 & $0.3 \%$ & $1.1 \%$ & $2.7 \%$ & $1.4 \%$ & $1.4 \%$ & $1.4 \%$ \\
\hline 55 & $0.3 \%$ & $0.4 \%$ & $2.3 \%$ & $2.0 \%$ & $2.0 \%$ & $1.3 \%$ \\
\hline 60 & $1.2 \%$ & $0.4 \%$ & $1.2 \%$ & $2.7 \%$ & $2.4 \%$ & $1.5 \%$ \\
\hline 65 & $1.6 \%$ & $0.6 \%$ & $0.8 \%$ & $2.9 \%$ & $3.0 \%$ & $1.6 \%$ \\
\hline 70 & $1.7 \%$ & $1.1 \%$ & $1.1 \%$ & $2.0 \%$ & $3.1 \%$ & $1.7 \%$ \\
\hline 75 & $1.7 \%$ & $1.3 \%$ & $1.3 \%$ & $1.6 \%$ & $2.3 \%$ & $1.6 \%$ \\
\hline 80 & $1.6 \%$ & $0.8 \%$ & $1.6 \%$ & $1.4 \%$ & $1.9 \%$ & $1.4 \%$ \\
\hline 85 & $1.5 \%$ & $0.8 \%$ & $1.2 \%$ & $1.0 \%$ & $1.6 \%$ & $1.2 \%$ \\
\hline Average & $1.1 \%$ & $1.8 \%$ & $1.7 \%$ & $1.8 \%$ & $1.7 \%$ & $1.6 \%$ \\
\hline
\end{tabular}

For notes see page 110 . 
Table 4.5: Average age-standardised annual improvement in mortality rates (QXI) by country and decade for five year age bands starting at age shown, continued

\begin{tabular}{|c|c|c|c|c|c|c|}
\hline \multicolumn{7}{|c|}{ US, males } \\
\hline Age & $\begin{array}{l}\text { 1961- } \\
71\end{array}$ & $\begin{array}{l}1971- \\
81\end{array}$ & $\begin{array}{l}\text { 1981- } \\
91\end{array}$ & $\begin{array}{l}1991- \\
2001\end{array}$ & $\begin{array}{l}1996- \\
2006\end{array}$ & $\begin{array}{l}1961- \\
2006\end{array}$ \\
\hline 0 & $2.0 \%$ & $3.3 \%$ & $2.6 \%$ & $3.3 \%$ & $2.9 \%$ & $2.8 \%$ \\
\hline 5 & $0.6 \%$ & $3.5 \%$ & $3.4 \%$ & $3.7 \%$ & $3.5 \%$ & $2.7 \%$ \\
\hline 10 & $0.8 \%$ & $2.9 \%$ & $1.4 \%$ & $3.3 \%$ & $3.5 \%$ & $2.2 \%$ \\
\hline 15 & $-2.5 \%$ & $2.4 \%$ & $-0.1 \%$ & $3.3 \%$ & $2.2 \%$ & $0.8 \%$ \\
\hline 20 & $-2.3 \%$ & $1.7 \%$ & $1.2 \%$ & $1.7 \%$ & $0.0 \%$ & $0.4 \%$ \\
\hline 25 & $-1.8 \%$ & $0.6 \%$ & $0.4 \%$ & $2.9 \%$ & $0.2 \%$ & $0.3 \%$ \\
\hline 30 & $-1.2 \%$ & $1.5 \%$ & $-1.4 \%$ & $3.8 \%$ & $2.4 \%$ & $0.6 \%$ \\
\hline 35 & $-1.0 \%$ & $2.7 \%$ & $-1.7 \%$ & $2.7 \%$ & $2.9 \%$ & $0.9 \%$ \\
\hline 40 & $-0.2 \%$ & $2.6 \%$ & $0.1 \%$ & $1.4 \%$ & $1.9 \%$ & $1.0 \%$ \\
\hline 45 & $-0.1 \%$ & $2.5 \%$ & $1.6 \%$ & $0.6 \%$ & $0.9 \%$ & $1.1 \%$ \\
\hline 50 & $0.6 \%$ & $2.2 \%$ & $2.1 \%$ & $1.3 \%$ & $0.2 \%$ & $1.3 \%$ \\
\hline 55 & $-0.1 \%$ & $2.3 \%$ & $1.9 \%$ & $2.0 \%$ & $1.4 \%$ & $1.4 \%$ \\
\hline 60 & $0.4 \%$ & $2.2 \%$ & $1.5 \%$ & $2.1 \%$ & $2.3 \%$ & $1.6 \%$ \\
\hline 65 & $0.3 \%$ & $1.9 \%$ & $1.6 \%$ & $1.9 \%$ & $2.2 \%$ & $1.5 \%$ \\
\hline 70 & $-0.3 \%$ & $1.6 \%$ & $1.6 \%$ & $1.7 \%$ & $2.4 \%$ & $1.3 \%$ \\
\hline 75 & $-0.1 \%$ & $1.6 \%$ & $1.2 \%$ & $1.3 \%$ & $1.9 \%$ & $1.2 \%$ \\
\hline 80 & $0.4 \%$ & $1.0 \%$ & $0.9 \%$ & $1.4 \%$ & $1.6 \%$ & $1.0 \%$ \\
\hline 85 & $0.4 \%$ & $1.1 \%$ & $0.6 \%$ & $1.2 \%$ & $2.0 \%$ & $0.9 \%$ \\
\hline Average & $-0.3 \%$ & $2.3 \%$ & $1.0 \%$ & $2.4 \%$ & $1.9 \%$ & $1.3 \%$ \\
\hline
\end{tabular}

For notes see page 110 . 
Table 4.5: Average age-standardised annual improvement in mortality rates (QXI) by country and decade for five year age bands starting at age shown, continued

\begin{tabular}{|c|c|c|c|c|c|c|}
\hline \multicolumn{7}{|c|}{ US, females } \\
\hline Age & $\begin{array}{l}\text { 1961- } \\
71\end{array}$ & $\begin{array}{l}1971- \\
81\end{array}$ & $\begin{array}{l}1981- \\
91\end{array}$ & $\begin{array}{l}1991- \\
2001\end{array}$ & $\begin{array}{l}1996- \\
2006\end{array}$ & $\begin{array}{l}1961- \\
2006\end{array}$ \\
\hline 0 & $2.2 \%$ & $3.6 \%$ & $2.3 \%$ & $3.3 \%$ & $2.6 \%$ & $2.8 \%$ \\
\hline 5 & $1.0 \%$ & $3.9 \%$ & $2.3 \%$ & $2.7 \%$ & $2.8 \%$ & $2.5 \%$ \\
\hline 10 & $0.3 \%$ & $3.2 \%$ & $2.0 \%$ & $1.5 \%$ & $3.2 \%$ & $1.9 \%$ \\
\hline 15 & $-1.9 \%$ & $2.4 \%$ & $0.4 \%$ & $2.0 \%$ & $1.9 \%$ & $0.8 \%$ \\
\hline 20 & $-1.0 \%$ & $2.2 \%$ & $1.2 \%$ & $1.2 \%$ & $-0.4 \%$ & $0.7 \%$ \\
\hline 25 & $0.0 \%$ & $2.4 \%$ & $0.7 \%$ & $1.2 \%$ & $0.4 \%$ & $0.9 \%$ \\
\hline 30 & $0.4 \%$ & $3.3 \%$ & $-0.2 \%$ & $1.1 \%$ & $1.4 \%$ & $1.1 \%$ \\
\hline 35 & $0.1 \%$ & $3.8 \%$ & $0.3 \%$ & $-0.1 \%$ & $1.0 \%$ & $1.1 \%$ \\
\hline 40 & $-0.1 \%$ & $3.5 \%$ & $1.5 \%$ & $-0.7 \%$ & $0.0 \%$ & $1.0 \%$ \\
\hline 45 & $0.1 \%$ & $2.7 \%$ & $1.8 \%$ & $0.1 \%$ & $-0.3 \%$ & $1.0 \%$ \\
\hline 50 & $0.4 \%$ & $2.1 \%$ & $1.4 \%$ & $1.0 \%$ & $0.4 \%$ & $1.1 \%$ \\
\hline 55 & $0.0 \%$ & $1.7 \%$ & $0.9 \%$ & $1.3 \%$ & $1.3 \%$ & $1.0 \%$ \\
\hline 60 & $1.2 \%$ & $1.4 \%$ & $0.7 \%$ & $1.0 \%$ & $1.8 \%$ & $1.2 \%$ \\
\hline 65 & $1.2 \%$ & $1.4 \%$ & $0.6 \%$ & $0.7 \%$ & $1.5 \%$ & $1.1 \%$ \\
\hline 70 & $0.9 \%$ & $2.0 \%$ & $0.7 \%$ & $0.6 \%$ & $1.4 \%$ & $1.1 \%$ \\
\hline 75 & $1.1 \%$ & $2.4 \%$ & $0.7 \%$ & $0.2 \%$ & $1.1 \%$ & $1.2 \%$ \\
\hline 80 & $1.2 \%$ & $1.8 \%$ & $1.2 \%$ & $0.2 \%$ & $0.8 \%$ & $1.2 \%$ \\
\hline 85 & $1.0 \%$ & $1.8 \%$ & $1.0 \%$ & $0.2 \%$ & $1.0 \%$ & $1.0 \%$ \\
\hline Average & $0.3 \%$ & $2.7 \%$ & $1.1 \%$ & $1.1 \%$ & $1.2 \%$ & $1.3 \%$ \\
\hline
\end{tabular}

For notes see page 110. 
The detailed data by country in Table 4.5 shows some similarity in patterns of age difference in mortality rates over the different time periods. The 'normal' pattern for these countries is a diagonal of highlighted cells centred within age bands starting at ages 45 to 55 in 1981-91 for males and ages 40 to 55 for females. In addition, most countries have high mortality improvement rates at young ages, around age 20-30 since 1991 for both males and females, and at older ages, 65 to 85 in the earliest decade of this analysis for females. The UK is unusual in not having either of these patterns for young or old ages. New Zealand is unusual in additionally having significantly high QXI for the age 85 age band in the 1960s. In the following, the diagonal pattern is discussed first, followed by the patterns at young and old ages.

\section{The diagonal: the golden cohort}

The diagonal provides the evidence for the 'golden cohort' already discussed in section 3.4: the cohort of people born just before and during World War II who have experienced higher rates of mortality improvement (QXI) than people born before or after them in every time period since birth. More precisely, the highlighted cells in this analysis identify at which adult ages there was a significantly high QXI among all adult QXI in each period, and the diagonal imputes the potential existence of a golden cohort. The golden cohort has been analysed in detail for the UK (or one region within the UK, England and Wales), and New Zealand, with some references to other countries (Andreev and Vaupel 2005; Dunnell 2008; Gallop 2008; Murphy 2010; O'Connell and Dunstan 2009; Willets et al. 2004). This analysis extends consideration of the existence of the golden cohort to a wider context of British 
settler countries; and additionally comments on its interpretation where there are significant rates of mortality improvement at other ages.

The cohort diagonal can be seen in all countries, for both genders, although faintly for some. It is strongest for the UK males and females, as there are no cells highlighted off the diagonal. In most cases, the diagonal extends across the whole time period. Even if not all the cells on the implied diagonal are highlighted, inspection of the data reveals that the QXI are high, and close to the average plus one standard deviation used as an indicator. The UK has the strongest golden cohort as the highlighted cells make a tidy diagonal, although even here it falters between the first and second decades. This confirms the experience of the golden cohort noted in the UK; that the cohort has kept their mortality improvement advantage throughout life.

Looking at the decade 1981-91 only, the ages of the clearest golden cohort for each country vary within the range of age bands starting 45 to 55 for males and ages 40 to 55 for females at that time. Table 4.6 summarises the approximate birth dates of the golden cohort estimated by this analysis for each country. As a compelling indication that other countries share the golden cohort hitherto given attention only in the $\mathrm{UK}$, the range implied across all countries is very close to that taken as the definition of the golden cohort and used in official mortality projections in the UK, that is birth years 1923 to 1940. For males, the golden cohorts of the UK and US appear the oldest. For females, the oldest would be in the UK, but the youngest complete cohort is in Australia and New Zealand. 
Table 4.6: Approximate birth years of golden cohort, by country and gender

\begin{tabular}{|lcc|}
\hline & Males & Females \\
\cline { 2 - 3 } Australia & $1922-1936$ & $1932-1936$ \\
Canada & $1922-1936$ & $1927-1941$ \\
New Zealand & $1927-1936$ & $1932-1936$ \\
UK & $1922-1931$ & $1922-1941$ \\
US & $1924-1931$ & $1927-1941$ \\
Range & $\mathbf{1 9 2 2 - 1 9 3 6}$ & $\mathbf{1 9 2 2 - 1 9 4 1}$ \\
\hline
\end{tabular}

Source: Possible birth years (inclusive) for age bands with significantly high rate of mortality improvement in decade 1981-91 indicated in Table 4.5. For calculation of significance see text.

\section{Young and old ages: not just the golden cohort}

The strength of the UK golden cohort is partly explained by the UK being unusual in not having significant QXI at young or old ages in the pattern shared by other countries of this analysis. All other countries have had significant mortality improvements at young ages for males and females in the decades starting 1991 or 1996 and at old ages for females in the decades starting in 1961. The significant QXI at old ages is largely of historical interest, as the youngest group showing this effect is the US female cohort born in 18971901. The significant QXI at young ages in recent decades are more relevant for considerations of future mortality as they may indicate the beginnings of a new golden cohort for people born in the years shown in Table 4.7. 
Table 4.7: Approximate birth years of possible new young ages golden cohort, by country and gender

\begin{tabular}{|lcc|}
\hline & Males & Females \\
\cline { 2 - 3 } Australia & $1967-1976$ & $1962-1966$ \\
Canada & $1957-1971$ & $1962-1966$ \\
New Zealand & $1967-1971$ & $1967-1976$ \\
UK & $\mathrm{n} / \mathrm{a}$ & $\mathrm{n} / \mathrm{a}$ \\
US & $1952-1966$ & $1967-1971$ \\
Range & $\mathbf{1 9 5 2 - 1 9 7 6}$ & $\mathbf{1 9 6 2 - 1 9 7 6}$ \\
\hline
\end{tabular}

Source: Possible birth years (inclusive) for age bands with significantly high rate of mortality improvement in decade 1991-2001 or 1996-2006 indicated in Table 4.5. For calculation of significance see text. Approximate only as an indication for a golden cohort.

This analysis is far from predicting further golden cohorts as there are insufficient time periods of data on which to base such a conclusion. However, it is suggestive enough for the possibility to be worth considering for projections of future mortality. It is consistent with the analysis of Murphy (2010) who suggested that a more interesting question for the UK than why the golden cohort existed was why other ages had missed out on such high mortality improvement rates. Indeed, not only does the UK miss out on the putative younger golden cohort on this definition, but the absolute level of mortality rate improvement at these ages in the UK appears low relative to what has been achieved in other countries. For example, the UK male QXIs in age bands 20 to 35 in the decade 1996-2006 range from 0.2 per cent to 2.1 per cent, but in Australia they range between 2.6 per cent and 4.5 per cent, in Canada 1.8 per cent to 3.9 per cent and in New Zealand 1.0 per cent to 3.7 per cent. Further, looking at QXI for age bands 5 to 15, the UK appears to have the lowest absolute values. Therefore, whether or not any new young golden cohorts are confirmed with later data, there is greater momentum for mortality 
improvement in cohorts younger than the established golden cohort in all these countries, including New Zealand, compared to the UK.

The final column in Table 4.5 identifies the adult age bands that experienced significantly faster mortality improvement compared to other adult ages over the whole forty-five year period. For males, the significant QXI age bands are between starting age 50 and 65 for all countries. The female significant QXI are more variable, being the band starting age 65 in Australia, 65 to 75 in the UK, and age 75 only for the US and New Zealand. In Canada, the most significant QXI were at age bands starting 25 and 30. This again confirms that New Zealand has had strong QXI at older ages compared to other countries. Inspection of QXI in the latest decade for the oldest three age bands, older than the golden cohort, shows that New Zealand's lags only Australia's.

Another way of looking at improvement in mortality rates over time is to consider trends in the summary mortality measure, life expectancy. The rate of improvement is a function of both the rate of improvement in mortality rates at each age and the weighting that each age takes in the life expectancy calculation. Table 4.8 shows the rate of improvement over the analysis period in life expectancy at birth, and at older ages for the selected countries. 
Table 4.8: Improvement in life expectancy at birth, at age 65 and at age 80 in selected countries, 1961 to 2006, in years and per cent

\begin{tabular}{|lcccccc|}
\hline & At birth & \multicolumn{3}{c}{ At age 65 } & \multicolumn{3}{c|}{ At age 80 } \\
Years & Females & Males & Females & Males & Females & Males \\
\cline { 2 - 7 } Australia & 9.3 & 11.1 & 5.8 & 6.0 & 2.9 & 2.5 \\
Canada & 8.5 & 9.9 & 4.9 & 4.6 & 2.9 & 2.0 \\
NZ & 8.4 & 9.6 & 5.2 & 5.2 & 3.1 & 2.5 \\
UK & 7.9 & 9.4 & 4.9 & 5.4 & 2.9 & 2.5 \\
US & 7.0 & 8.5 & 4.0 & 4.4 & 2.6 & 2.3 \\
& & & & & & \\
Per cent & & & & & & \\
Australia & $12 \%$ & $16 \%$ & $36 \%$ & $48 \%$ & $42 \%$ & $43 \%$ \\
Canada & $11 \%$ & $14 \%$ & $30 \%$ & $34 \%$ & $39 \%$ & $32 \%$ \\
NZ & $11 \%$ & $14 \%$ & $33 \%$ & $40 \%$ & $47 \%$ & $46 \%$ \\
UK & $11 \%$ & $14 \%$ & $33 \%$ & $45 \%$ & $45 \%$ & $48 \%$ \\
US & $9 \%$ & $13 \%$ & $25 \%$ & $34 \%$ & $36 \%$ & $38 \%$ \\
& & & & & & \\
\hline
\end{tabular}

Source: Calculated from life table data. New Zealand data from Statistics New Zealand life tables. Other mortality rates from Human Mortality Database life tables (downloaded August 2010).

New Zealand's rate of improvement of life expectancy at birth is consistent with the foregoing mortality analysis. New Zealand's rate of improvement of life expectancy at birth is less than Australia's, on a par with Canada's and ahead of that in the UK and US. By age 65 New Zealand's life expectancy improvement rate is pulling ahead of all countries including, by age 80, Australia. This comparison suggests that New Zealand's rate of mortality improvement at the oldest ages has been very strong. 
The overall pattern by age may in part reflect the focus of medical technologies and the impact of public health initiatives. The attention of both these factors has been concentrated at young ages (the care of newborns and very young children) and at middle to older ages (progress in the prevention and treatment of cancers and cardiovascular disease). At teenage and young adult ages, mortality rates are low and relatively more subject to accident rates rather than health issues. The rankings of mortality improvement of each country at each age group could reflect varying success rates of different medical technologies or public health initiatives.

Alternatively, there could be an underlying environmental cause elevating or depressing QXI at all ages. The sustained positions of Australia and the US across age bands point to this. The US health problem was discussed earlier. The reasons why Australia has sustained a relatively low mortality trend have not been subject to such scrutiny. Contributory factors may include a healthy environment, high level of economic development and associated good quality healthcare or a high level of people born overseas bringing with them selectively good mortality (see Table 3.4). These factors are to some extent shared with New Zealand. Given the proximity of the two countries, Australian mortality appears an appropriate comparator for New Zealand. 


\subsection{Median and modal age at death}

Similar patterns for the change in shape of the curve of deaths and the convergence of the three measures of central tendency exist for both males and females in the selected countries of this analysis. Figure 4.9 shows the curve of deaths for males in the selected countries for 1961, restricted to ages 56 to 95 to allow the detail of the adult modal peak. Figure 4.10 shows the same for 2006 .

Figure 4.9: Curve of deaths for males from 100,000 births, from period table 1961, selected countries, ages 56 to 95

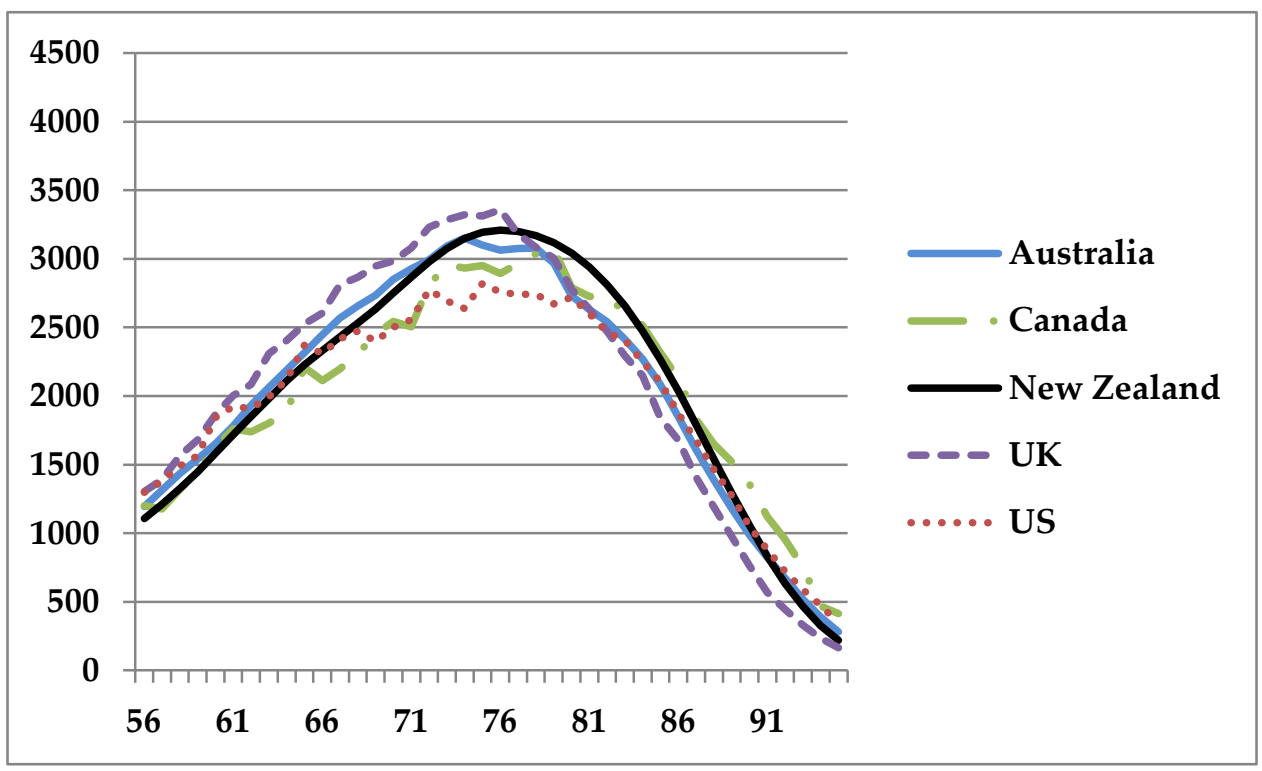

Source: Calculated from period life table data. New Zealand data from Statistics New Zealand life tables. Other mortality rates from Human Mortality Database life tables (downloaded August 2010). 
Figure 4.10: Curve of deaths for males from 100,000 births, 2006, selected countries, ages 56 to 95

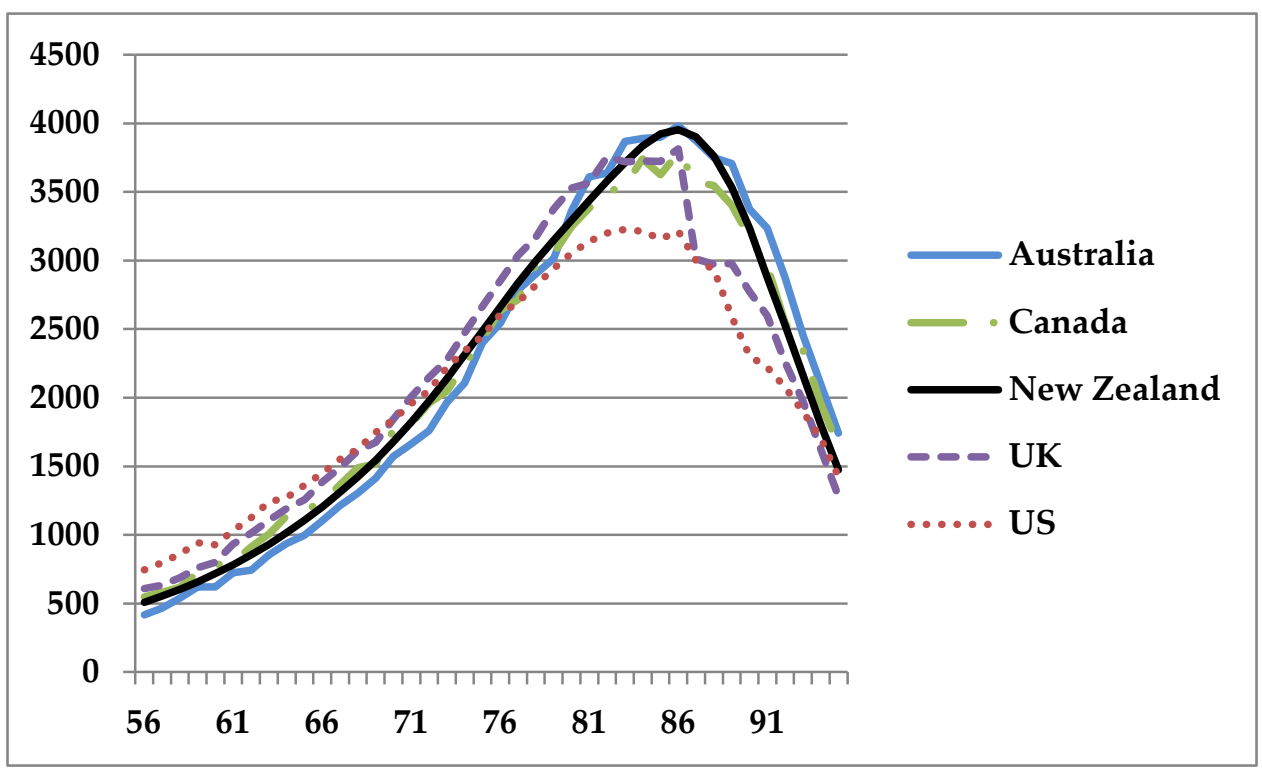

Source: As Figure 4.9 except UK figures revised November 2010 to smooth an obvious anomaly in $d_{x}$ at ages 86-7 (data still appear somewhat unsmoothed).

The consistent change in shape across countries is evident, but the consistency prevents more insightful comparison. Following the suggestion of Canudas-Romo (2010), a simultaneous comparison of the three measures of central tendency, and the rate of change of each, is provided by Table 4.9. The rate of improvement in life expectancy at birth was shown in Table 4.8, and is also included here.

The analysis of the improvements by age in mortality rate in the previous section showed that New Zealand has had particularly strong improvement in mortality over age 40 over the full period considered. The comparison of improvements in life expectancy at age 80 suggested New Zealand has had comparatively strong improvement at the oldest ages. Because the modal age at death is particularly affected by changes in mortality at ages above the 
measure (see Table 3.2), a strong improvement in the mode indicates strong increase in mortality rates above the modal age. Table 4.9 does indeed show a comparatively strong rate of improvement for New Zealand in modal age at death. New Zealand has the highest female modal age at death and third highest male mode, only slightly behind Australia and Canada. New Zealand's percentage increase in modal age ranks second to Australia and equal to that of the UK. The significance of this is indicated by the greater range in improvement rates of modal age at death across the countries $(7$ percentage points for males; 6 percentage points for females) compared to that for average and median ( 3 percentage points only). The larger range is itself indicative of the current momentum in QXI at the oldest ages. 
Table 4.9: Three measures of central tendency for the age at death distribution and the increase in years and per cent, 1961 to 2006, selected countries

\begin{tabular}{|lccccc|}
\hline & Australia & Canada & NZ & UK & US \\
\cline { 2 - 6 } Life expectancy at birth (average age at death) & & \\
Males & & & & & \\
$\mathbf{1 9 6 1}$ & 68.1 & 68.4 & 68.4 & 67.8 & 67.0 \\
$\mathbf{2 0 0 6}$ & 79.2 & 78.3 & 78.0 & 77.2 & 75.5 \\
Increase & 11.1 & 9.9 & 9.6 & 9.4 & 8.5 \\
& $16 \%$ & $14 \%$ & $14 \%$ & $14 \%$ & $13 \%$ \\
Females & & & & & \\
$\mathbf{1 9 6 1}$ & 74.5 & 74.5 & 73.8 & 73.7 & 73.7 \\
$\mathbf{2 0 0 6}$ & 83.8 & 82.9 & 82.2 & 81.5 & 80.7 \\
Increase & 9.3 & 8.5 & 8.4 & 7.9 & 7.0 \\
& $12 \%$ & $11 \%$ & $11 \%$ & $11 \%$ & $9 \%$ \\
Median age at death & & & & \\
Males & & & & & \\
$\mathbf{1 9 6 1}$ & 71.8 & 72.9 & 72.6 & 71.3 & 71.1 \\
$\mathbf{2 0 0 6}$ & 82.4 & 81.5 & 81.5 & 80.3 & 79.2 \\
Increase & 10.6 & 8.7 & 8.9 & 8.9 & 8.2 \\
& $15 \%$ & $12 \%$ & $12 \%$ & $13 \%$ & $12 \%$ \\
Females & & & & & \\
$\mathbf{1 9 6 1}$ & 78.3 & 78.6 & 77.9 & 77.4 & 78.1 \\
$\mathbf{2 0 0 6}$ & 86.7 & 86.0 & 85.4 & 84.4 & 84.0 \\
Increase & 8.4 & 7.4 & 7.5 & 6.9 & 5.9 \\
& $11 \%$ & $9 \%$ & $10 \%$ & $9 \%$ & $8 \%$ \\
Modal age at death & & & & \\
Males & & & & & \\
$\mathbf{1 9 6 1}$ & 74.5 & 79.1 & 76.6 & 76.2 & 75.7 \\
$\mathbf{2 0 0 6}$ & 86.4 & 86.4 & 86.4 & 86.1 & 83.6 \\
Increase & 11.9 & 7.3 & 9.7 & 9.9 & 7.9 \\
Females & $16 \%$ & $9 \%$ & $13 \%$ & $13 \%$ & $10 \%$ \\
$\mathbf{1 9 6 1}$ & 79.2 & 84.3 & 81.8 & 79.5 & 83.5 \\
$\mathbf{2 0 0 6}$ & 88.6 & 89.7 & 89.3 & 86.4 & 88.3 \\
Increase & 9.5 & 5.4 & 7.5 & 6.9 & 4.8 \\
& $12 \%$ & $6 \%$ & $9 \%$ & $9 \%$ & $6 \%$ \\
\hline
\end{tabular}

Source: see Figure 4.10. 


\subsection{Conclusions}

Empirical data on the level of mortality rates and life expectancy at birth from the most current data and forty-five years earlier show New Zealand in the middle of the selected group of comparator countries. There is slightly better mortality in Canada and obviously better mortality in Australia. There is slightly worse mortality in the UK and obviously worse mortality in the US. Mortality in New Zealand is relatively better at ages over 40 than below, and this strength has become more apparent over the last forty-five years. New Zealand compares least well for mortality at ages 1-20 for both males and females indicating a problem with accidental deaths at young ages. New Zealand is notable for high modal age at death, indicating relatively low mortality at ages over 80 .

The selected countries share a similar picture of mortality rate development over the period 1961-2006. Mortality rates have continuously improved and female mortality rates are staying below those for males. Australia and Canada are examples of low mortality/high life expectancy/fast improvement countries; the US is higher mortality/lower life expectancy/slower improvement, albeit with apparently better mortality at the highest ages which is likely due to country-specific features. New Zealand and the UK rank in the middle of the group for the pace of mortality improvement. In the UK mortality improvement has been strong, especially at the 'golden cohort' of those now aged over 60 . However, New Zealand has shown even stronger mortality improvement more recently. The persistent rate of mortality change confirms that past period life expectancies cannot be a relevant contemporary lifespan measure. 
This analysis has built on previous research to draw new conclusions about 'golden cohorts' in all the countries analysed. The selected countries share to a greater or lesser significance a golden cohort who have experienced throughout their adult life in this time period significantly higher mortality improvement rates than immediately older or younger generations. The golden cohort are generally aged 45 to 55 in 1981-91 for males and ages 40 to 55 for females. In addition, most countries have had high mortality improvement rates for a younger cohort born around the 1960s and early 1970s and also for an older cohort of females aged around 65 to 85 in the 1960s. The UK is unusual as both these patterns for young and old ages are absent while the main golden cohort appears more strongly. New Zealand is unusual for additionally having had significantly high mortality improvement rates for the age 85 age band in the 1960s.

For this group of countries, the general shapes of all life table measures are similar and the pattern of comparison between countries consistent, which means that a difference in one country is notable. Where the patterns can be explained, hypotheses for future trends can be developed. For example, it has been suggested that because a large part of the gap in life expectancy between the US and other high-income countries appears to be caused by smoking, so future relative improvement in US longevity can be expected with a time lag reflecting the pace at which smoking prevalence declined. However, prevalence of smoking and the highly correlated death rate from lung cancer can both be measured, whereas other causal factors for specific causes of death can only be hypothesised. This analysis does not therefore allow specific future mortality trends for any one country to be identified. 
However, this analysis does identify new insights about New Zealand's mortality that can be used to enhance extrapolative methods of projecting future mortality. The comparison with other countries suggests the most similar countries whose mortality trends New Zealand may follow. The country with the closest level and average improvement rate of mortality to New Zealand's has been the UK. However, since the 1980s for females and the 1990s for males, New Zealand's rates of mortality improvement have been higher than the UK's and more like Australia's. As the rate of mortality improvement is the basis for future mortality projection, it would appear that the UK and Australia have the best claims as models for New Zealand.

In addition, this analysis isolates notable features of New Zealand's mortality that can be specifically allowed for in future projections. These features include that New Zealand has had relatively strong recent momentum in mortality improvement over the forty-five year period at older ages: over 40 and especially over 80 . This positive trend is diminished by relatively poor mortality at ages 1-20, although mortality improvement has been relatively strong for ages 15-25 in the last decade. The role of these and other factors is developing scenarios for New Zealand's future mortality is discussed in the next chapter. 
Underestimating lifespans? Why longevity risk exists Chapter 4

This page has been intentionally left blank. 


\section{Chapter 5: Future longevity in New Zealand}

This chapter reports on the second part of the analytic framework developed in Chapter 3. Building on the insights in the last chapter from setting past mortality in New Zealand in the context of that in like countries, the mortality projections of the selected countries are now compared. The aim is to test whether the set of assumptions underlying the future projections from the group of selected countries is internally consistent and plausible. The question is not whether each country has followed a suitable method of projection, but rather, given the relative performance of past mortality trends do the relative projected mortality trends appear reasonable? In particular, what does this imply for New Zealand's mortality projections: do they appear relatively optimistic or pessimistic against the projections of other countries?

This chapter first compares the assumptions made in the mortality projections of each of the comparator countries. It then compares the results of those projections. The third section reflects New Zealand's relative past mortality experience in the context of the relativities in the national projections. The next section considers potential adverse trends before a concluding section draws together findings to reinterpret existing New Zealand mortality projections. 


\subsection{Assumptions in international mortality projections}

This section sets out what is known about the assumptions made by each agency making the actuarial mortality projections selected in section 3.5. Extrapolative methods are used for all these projections. Although the detail of the extrapolation varies, the fundamental building blocks of each mortality projection are the same: the annual rate of improvement of age-specific mortality rates - QXI, as defined in section 3.4. In New Zealand, the US and UK mortality projections are published on at least three sets of assumptions or variants, resulting in 'high', 'medium' and 'low' projections. Australia publishes only two variant projections, and Canada just one set. Statistics New Zealand's labelling is dropped in this chapter as other countries usually label their variant projections by the outcome of mortality assumptions on life expectancy. So in this chapter, a 'high' projection refers to a low mortality assumption resulting in high life expectancy (Low Mortality in the terminology used elsewhere in this thesis for Statistics New Zealand's projection).

In order for each projection to result in future cohort life expectancy measures, mortality rates for each age and sex need to be projected for each future year that members of the cohorts are alive. Each of the actuarial projections calculates mortality rates for each calendar year up to 2050 and some have projection periods which continue beyond. The mortality rates are calculated from the assumed QXI for each age, sex and year. The assumptions for QXI are informed by past QXI trends and by judgements on, for example: how long a period of past trends is extrapolated; whether QXI are made to be age- or cohort-specific throughout the period of projection, or whether some 
age groups share a QXI; whether QXI continue at the same rate for the course of the projection period in a straight line, or are shaped to a judgement that rates of improvement will lessen over time. It is therefore complex to describe each set of assumptions, but possible to summarise the pattern of QXI assumed.

In some cases, the assumption for future QXI is a single number, as the agency involved has made the judgement that QXI would be the same for all ages for a number of future years. For example, in the UK, a target QXI is assumed to apply for all ages from twenty five years out from the start of the projection period and for the remainder of the projection period. Before then, QXI vary by age in a way chosen so that the QXI of the last year with actual data transition to the target QXI on a path that reflects past age-specific past trends in QXI. The projections for Canada and the US take a similar approach with a slightly different target date and with two different QXI in the second period for ages above and below age 85 or age 65 respectively. Table 5.1 explains the assumptions for QXI in the selected mortality in a highly summarised way; Table 5.2 describes the rationale given for the choice of assumptions.

Each of the projections is illustrative rather than exact, and the variants within a country are arbitrary. As is apparent from Table 5.2, the assumptions underlying each projection depend on many judgements, the full complexity of which is not always explained in published reports. The rationale for a particular choice of assumption is often not described or insufficient information is available to enable it to be inferred. Yet as Table 5.1 reveals, the key assumption is often asserted as one, rounded number. For example, after 
the first twenty five years of the projection period in the UK the annual reduction in mortality rates is assumed to be a constant 0,1 or 2 per cent for all ages. Given the nature of mortality trends - that despite being influenced by a large number of processes operating somewhat randomly population average mortality has still declined in a fairly linear way - it is not surprising that decisions are taken to project mortality using a highly summarised straight line assumption. However, the great detail of past mortality experience data in combination with simplified assumptions can present the appearance of more accuracy in the projection that is actually present. All the projections are 'what-if' scenarios essentially assuming a simplified continuation of the historic average mortality trend. 
Table 5.1: Summarised assumptions for the annual rate of change in mortality rates in population projections of selected countries

\begin{tabular}{|c|c|c|c|}
\hline & Low LE variant & Medium projection & High LE variant \\
\hline Australia & $1.8 \%(\mathrm{~m}) 2.2 \%(\mathrm{f})$ & $\mathrm{n} / \mathrm{a}$ & $2.7 \%(\mathrm{~m}) 2.2 \%(\mathrm{f})$ \\
\hline Canada & $\mathrm{n} / \mathrm{a}$ & $\begin{array}{l}\text { Varies by age to } \\
2029 \text { then } 0.7 \% \text { for } \\
\text { all ages under } 85, \\
\text { lower for ages } 85 \\
\text { and over }\end{array}$ & $\mathrm{n} / \mathrm{a}$ \\
\hline $\begin{array}{l}\text { New } \\
\text { Zealand }\end{array}$ & $1 \%(\mathrm{~m}) 1.3 \%(\mathrm{f})$ & $1.6 \%(\mathrm{~m}) 1.8 \%(\mathrm{f})$ & $2.1 \%(\mathrm{~m}) 2.4 \%(\mathrm{f})$ \\
\hline UK & $\begin{array}{l}\text { Varies by age until } \\
\text { reaches } 0 \% \text { by } 2032 . \\
\text { Thereafter, } 0 \% \text { all } \\
\text { ages }\end{array}$ & $\begin{array}{l}\text { Varies by age until } \\
\text { reaches } 1 \% \text { by } 2032 . \\
\text { Thereafter, } 1 \% \text { all } \\
\text { ages. } \\
\text { Average } 2008-2083 \text { : } \\
1.3 \% \text { (m) } 1.4 \% \text { (f) }\end{array}$ & $\begin{array}{l}\text { Varies by age until } \\
\text { reaches } 2 \% \text { by } 2032 . \\
\text { Thereafter, } 2 \% \text { all } \\
\text { ages }\end{array}$ \\
\hline US & $\begin{array}{l}\text { Varies by age to } \\
2034 \text { then } 0.5 \% \text { for } \\
\text { all ages under } 65, \\
0.3 \% \text { for ages } 65 \\
\text { and over }\end{array}$ & $\begin{array}{l}\text { Varies by age to } \\
2034 \text { then } 1 \% \text { for all } \\
\text { ages under } 65,0.7 \% \\
\text { for ages } 65 \text { and over }\end{array}$ & $\begin{array}{l}\text { Varies by age to } \\
2034 \text { then } 1.5 \% \text { for } \\
\text { all ages under } 65 \text {, } \\
1.2 \% \text { for ages } 65 \text { and } \\
\text { over }\end{array}$ \\
\hline
\end{tabular}

Note: The figures shown in the above are those given by the statistical agency for the annual rate of change in mortality rates, averaged over ages and time periods, for the duration of the projection period or part-period as shown. For Australia, averages weighted by population count of the given QXI have been calculated. $\mathrm{m}=$ male; $\mathrm{f}=$ female. Low and high variants named to reflect lower or higher life expectancy result (higher or lower mortality). These labels may not be those used in original cases and are not those used in New Zealand. Each country labels variants differently. Source: Extracted and summarised from source documents: AGA 2009; OASDI Board of Trustees 2010; OCA 2009; ONS 2009; Statistics New Zealand 2009a. Original analysis by author first published in O'Connell (2011b). 
Table 5.2: Given rationale for the assumptions underlying population projections of selected countries

\begin{tabular}{|c|c|}
\hline & $\begin{array}{l}\text { Summary comment on assumptions for annual rate of } \\
\text { mortality improvement (QXI) for medium projection, with } \\
\text { high and low as variations (where relevant) }\end{array}$ \\
\hline Australia & $\begin{array}{l}\text { Historic average age-specific QXI applied to current qx (2005- } \\
\text { 7) cumulatively for each future year of projection period. } \\
\text { Low variant uses average from } 100 \text { prior years. } \\
\text { High variant uses average from } 25 \text { prior years. }\end{array}$ \\
\hline Canada & $\begin{array}{l}\text { Future QXI set by judgement informed by historic time series } \\
\text { of age specific QXI 1989-2004. Pivot year set as } 2029 \text {. } \\
\text { For } 2029 \text { and after: QXI set equal for all ages below age } 85 \text {. } \\
\text { Until 2029: age-specific QXI set by linear interpolation } \\
\text { between relevant QXIs for } 2009 \text { and } 2029 \text {. }\end{array}$ \\
\hline $\begin{array}{l}\text { New } \\
\text { Zealand }\end{array}$ & $\begin{array}{l}\text { Future age-specific QXI } 2006 \text { and } 2061 \text { "essentially driven" by } \\
\text { age-specific death rates in cohort life tables for 1876-2007 } \\
\text { birth cohorts. Extrapolation by curve-fitting, but with some } \\
\text { judgements to give plausible death numbers by age-sex in the } \\
\text { initial years of the projection period. QXI vary by age and } \\
\text { future year of projection period. } \\
\text { High and low variants set by arbitrary }+/-3 \text { years (m) } 2.5 \\
\text { years (f) for period life expectancy at birth } 2061 \text {. } \\
\text { Additional "Very low mortality" variant asserts period life } \\
\text { expectancy at birth of } 95 \text { years by } 2061 \text {. }\end{array}$ \\
\hline UK & $\begin{array}{l}\text { Future QXI set by judgement informed by historic time series } \\
\text { of age specific QXI, primarily 1967-2007. } \\
\text { Pivot year set as 2032/3 when target QXI reached, same for all } \\
\text { ages thereafter. Target QXI of } 1 \text { per cent per year equal to the } \\
\text { average over whole of 20th century for both males and } \\
\text { females. }\end{array}$ \\
\hline
\end{tabular}

Table continues, with notes, on the following page. 
Table 5.2: Given rationale for the assumptions underlying population projections of selected countries, continued

\begin{tabular}{|l|l|}
\hline continued & $\begin{array}{l}\text { Summary comment on assumptions for annual rate of } \\
\text { mortality improvement (QXI) for medium projection, with } \\
\text { high and low as variations (where relevant) }\end{array}$ \\
\hline $\begin{array}{l}\text { Until 2032/3: QXI transitions each year from } 2008 \text { to target in } \\
\text { for females to reflect recent trends. Specific assumptions for } \\
\text { cohort born 1923-40 to allow continued relatively high } \\
\text { improvement rates. }\end{array}$ \\
$\begin{array}{l}\text { For low variant, target QXI set to zero (so mortality rates do } \\
\text { not change after 2032); high variant set equidistant from } \\
\text { medium projection at 2 per cent per year. }\end{array}$ \\
\hline US & $\begin{array}{l}\text { Future QXI set by judgement informed by historic time series } \\
\text { of age-specific QXI (1900-2006) and of cause of death trends } \\
\text { (1976-2006). } \\
\text { Pivot year set as 2034 when target QXIs reached, then kept } \\
\text { constant thereafter. QXI set for all ages below } 65 \text { at } 1 \text { per cent } \\
\text { per year and at 0.7 per cent per year for ages } 65 \text { and above, } \\
\text { reflecting slower reductions in historic mortality rates for } \\
\text { older ages. These QXI are on average lower than the average } \\
\text { QXI of 1900-2006 and for 1979-2006. } \\
\text { Until 2034: age-specific "rapid change" transition of QXI is } \\
\text { assumed from observed 2006 values to 2034 target. } \\
\text { For low and high variants, age-specific QXI levels are set at 50 } \\
\text { per cent or 150 per cent of the medium projection QXI. }\end{array}$ \\
\hline
\end{tabular}

Note: QXI is annual rate of mortality improvement. See section 3.4.

See also notes for Table 5.1.

Source: Extracted and summarised from source documents: AGA 2009; OASDI Board of Trustees 2010; OCA 2009; ONS 2009; Statistics New Zealand 2009a and personal communication Statistics New Zealand. 
Each projection is based on an assumption that age-specific mortality rates for both sexes decrease in future. Only the low variant in the UK is based on an assumption that after an initial period of improving mortality, the rate of improvement slows to zero. No projection involves mortality worsening in future. This means that lifespans are assumed to continue to lengthen over time. Differences between projections of different countries and between variants within a country are due to different assumptions on the pace and shape of future mortality improvement.

For each country, there is a rationale for the assumption based on actual past mortality in that country. However, the judgements behind each assumption mean that it is possible the choices made on mortality assumptions are made with varying degrees of optimism or pessimism. But it is not possible to identify this accurately. A direct comparison of the summary assumptions for future QXI for each country from Table 5.1 with the average of past QXI from Table 4.4 is not sufficient. The weightings over ages are not the same in each table, and the average QXI over the projection period in Table 5.1 are in most cases given without information on how the shape of QXI changes over time. However, the mortality projections for Australia and New Zealand appear to assume sustained mortality improvement at a higher rate than the other countries. The medium projection in Canada, the US and UK assumes that mortality improvement rates transition after a period to the order of 0.7 to 1 per cent per year. In the medium projection for New Zealand and in both projections in Australia the pace of improvement is sustained at rates around 1 per cent higher. This appears to suggest that the assumptions in Australia and New Zealand are relatively optimistic and consistent with an assumption that mortality rates in the selected countries would diverge; or 
that the assumptions for Canada in particular are relatively pessimistic, consistent with convergence of mortality rates.

A caveat to the above is that the shape of the QXI over the projection period will affect the resulting mortality rates and lifespan measures for future years. The summarised picture hides this detail, and the published reports of the projections do not give enough information to describe fully the path of QXI. However, a difference between countries in the assumed shape of future QXI is revealed. In the New Zealand projections, the average QXI for females over all ages and years 2006-61 is 1.8 per cent. But this has within it an assumption of increasing pace of improvement: the average QXI for 2006-31 is 1.7 per cent which rises to 2.0 per cent for the remainder of the projection period to 2061 (personal communication from Statistics New Zealand). For males, the assumption averaged over all ages for the two phases of the projection period are the same at 1.6 per cent. Conversely, in the UK, the QXI are generally higher prior to 2033 than later in the projection period (ONS 2009 p. 27). This assumption of 'flattening' QXI over time can be inferred to underlie the projections for Canada and the US, while Australia assumes a different pattern, with the same QXI applying each year of the projection period at each age. The agencies making assumptions about the future path of mortality hold different implicit or explicit views on the trajectory of that path. 


\subsection{International projections of lifespan}

More optimistic QXI assumed at the start of the projected period, especially at young ages, are likely to result in higher life expectancy measures than projections with less optimistic QXI at the start, even if these have higher QXI at the end of the period. Therefore, there is a limit to what can be interpreted by examination of projection assumptions. The results of the projections, which are measured by life expectancy, need to be examined. The fact that these countries all assume continued mortality improvement confirms that projected period life expectancies cannot be relevant future lifespan measures. This supports the use of cohort life expectancies to report on the results of the projections. However, the first illustration explores the summary commonly presented. Table 5.3 shows period life expectancy for the beginning of the projection period and a selected long-term point in 2050 from the medium projection only (both of the two Australian variants are shown as a single best estimate is not provided). 
Table 5.3: Period life expectancy at birth and at age 65 for years 2010 and 2050 from medium mortality projections in selected countries, in years

\begin{tabular}{|c|c|c|c|c|c|c|c|c|}
\hline & \multicolumn{4}{|l|}{2010} & \multicolumn{4}{|l|}{2050} \\
\hline & \multicolumn{2}{|l|}{ Male } & \multicolumn{2}{|c|}{ Female } & \multicolumn{2}{|l|}{ Male } & \multicolumn{2}{|c|}{ Female } \\
\hline & $\begin{array}{c}\text { At } \\
\text { birth }\end{array}$ & $\begin{array}{c}\text { At } \\
\text { age } \\
65\end{array}$ & $\begin{array}{c}\text { At } \\
\text { birth }\end{array}$ & $\begin{array}{c}\text { At } \\
\text { age } \\
65\end{array}$ & $\begin{array}{c}\text { At } \\
\text { birth }\end{array}$ & $\begin{array}{c}\text { At } \\
\text { age } \\
65\end{array}$ & $\begin{array}{c}\text { At } \\
\text { birth }\end{array}$ & $\begin{array}{c}\text { At } \\
\text { age } \\
65\end{array}$ \\
\hline Australia & & & & & & & & \\
\hline High & 80.1 & 19.2 & 84.4 & 22.1 & 88.3 & 25.0 & 90.5 & 26.7 \\
\hline Low & 79.5 & 18.6 & 84.2 & 21.9 & 84.0 & 21.5 & 88.3 & 24.7 \\
\hline $\begin{array}{l}\text { Canada } \\
\text { New }\end{array}$ & 79.2 & 18.7 & 83.2 & 21.4 & 83.0 & 21.3 & 86.0 & 23.4 \\
\hline Zealand & 78.8 & 18.6 & 82.5 & 20.8 & 84.4 & 22.2 & 87.5 & 24.4 \\
\hline UK & 78.7 & 18.5 & 82.6 & 20.8 & 85.0 & 23.5 & 88.5 & 25.8 \\
\hline US & 75.8 & 17.3 & 80.4 & 19.7 & 80.3 & 19.9 & 84.0 & 22.0 \\
\hline
\end{tabular}

Note: As there is no medium projection for Australia, both 'High' and 'Low' life expectancy variants are shown. See Table 5.1 and text for further explanation. Canada is Canada less Québec. UK 2010 data is for the year midpoint 2010 to midpoint 2011; for 2050 the annual average for 2046-2051 is given.

Source: Extracted from source documents and supplementary tables to: AGA 2009; OASDI Board of Trustees 2010; OCA 2009; ONS 2009 and personal communication from Statistics New Zealand for New Zealand data.

Inspection of the ranking of the measures in Table 5.3 shows that the assumptions made under the Australian 'High' variant imply Australia keeps its relative position as the highest period life expectancy at birth and at age 65 for both males and females over the period to 2050. However, the UK's medium projection overtakes the 'Low' variant for Australia. New Zealand's relative position improves and Canada's declines, while the US retains the lowest ranking. There is therefore a divergence in period life expectancy over the projection period across the countries. 
Divergence appears starkly in the comparison between the UK and New Zealand. In 2010, all the period life expectancy measures shown here are within 0.1 of a year across the two countries. However, by 2050, the UK measures are projected to overtake New Zealand's by up to one year for female life expectancy at birth and 1.4 years for female life expectancy at age 65. Relatively then, the assumptions underlying the UK medium projection are optimistic compared to those of New Zealand. Reference to Table 5.1 shows that it must be the greater momentum in QXI in the early projection years which making this difference, as the overall average QXI over the whole period are not strongly dissimilar. The mortality projections are highly sensitive to the QXI assumptions; both their level and their shape over the projection period. Yet, as Table 5.2 described, some assumptions are made arbitrarily.

Table 5.4 shows the implied increase in period life expectancy over the forty year projection period considered here. Leaving aside the high variant from Australia, the range of increases in life expectancy at birth is wide: 3.8 to 6.3 years for males and 2.8 to 5.9 years for females. The ranges of life expectancy at age 65 are even wider relative to the absolute levels: from 2.6 to 5.0 years for males and 2.0 to 5.0 years for females. 
Table 5.4: Increases in period life expectancy at birth and at age 65 from 2010 to 2050 from selected mortality projections, males and females, in years

\begin{tabular}{|c|c|c|c|c|}
\hline & \multicolumn{2}{|l|}{ Male } & \multicolumn{2}{|c|}{ Female } \\
\hline & $\begin{array}{c}\text { At } \\
\text { birth }\end{array}$ & $\begin{array}{c}\text { At } \\
\text { age } \\
65\end{array}$ & $\begin{array}{c}\text { At } \\
\text { birth }\end{array}$ & $\begin{array}{c}\text { At } \\
\text { age } \\
65 \\
\end{array}$ \\
\hline Australia & & & & \\
\hline High & 8.2 & 5.8 & 6.1 & 4.6 \\
\hline Low & 4.5 & 2.9 & 4.1 & 2.8 \\
\hline $\begin{array}{l}\text { Canada } \\
\text { New }\end{array}$ & 3.8 & 2.6 & 2.8 & 2.0 \\
\hline Zealand & 5.6 & 3.6 & 5.0 & 3.6 \\
\hline UK & 6.3 & 5.0 & 5.9 & 5.0 \\
\hline US & 4.5 & 2.6 & 3.6 & 2.3 \\
\hline
\end{tabular}

Notes and sources: See Table 5.3.

To investigate how relative positions of the measure more important for this study - average cohort lifespan - have changed in the past and could change in future, analysis now turns to using cohort measures of life expectancy. Figure 5.1 shows cohort life expectancies at birth in calendar year 2010 (so for the cohort born in 2010) and Figure 5.2 shows the same measure for births in calendar year 2050. Figure 5.3 shows cohort life expectancies at age 65 in 2010, that is, remaining lifespan as at 2010 for the cohort born in calendar year 1945. Figure 5.4 shows the same for calendar year 2050, referring to the cohort born in 1985 . These cohorts are not yet extinct, so require projections of future mortality. The range of projections shown for each selected country is consistent with the projection variants shown in Table 5.1 . 
Figure 5.1: Cohort life expectancy at birth in calendar year 2010 for selected countries, main projection variants

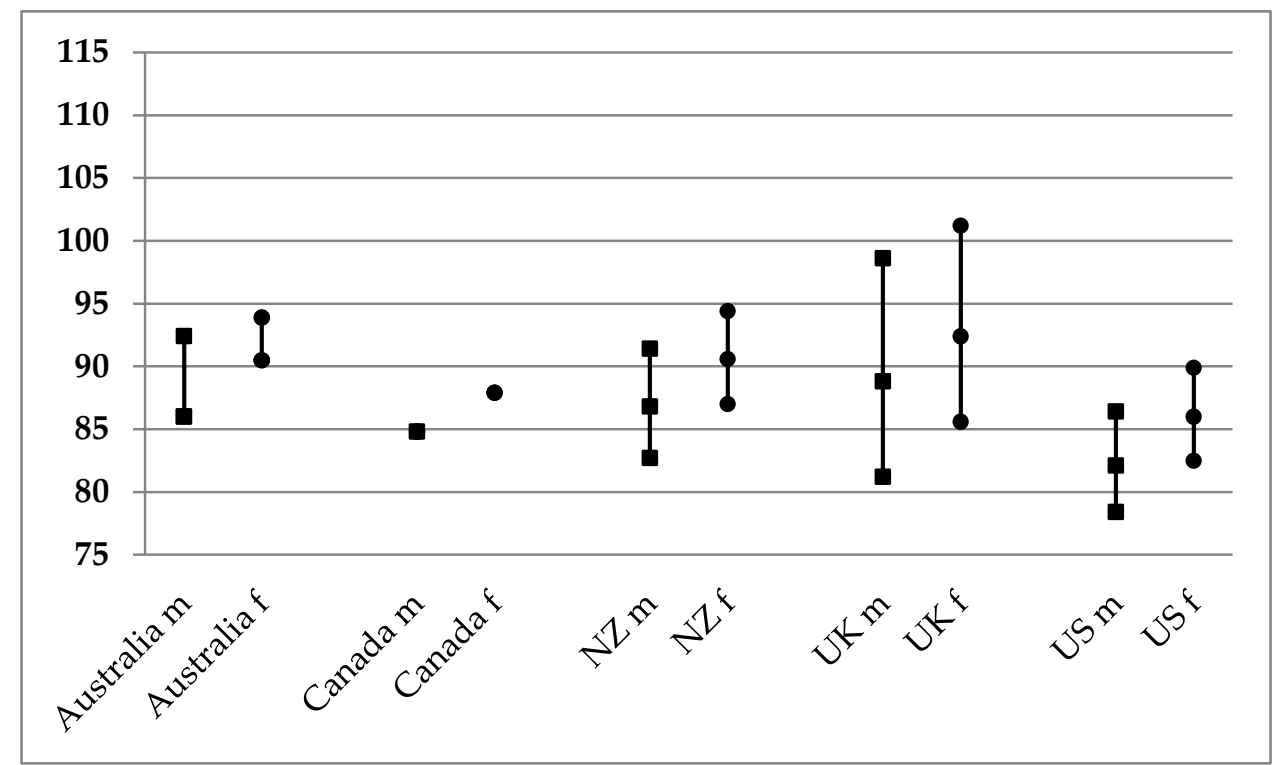

Source: See Table 5.3.

Figure 5.2: Cohort life expectancy at birth in calendar year 2050 for selected countries, main projection variants

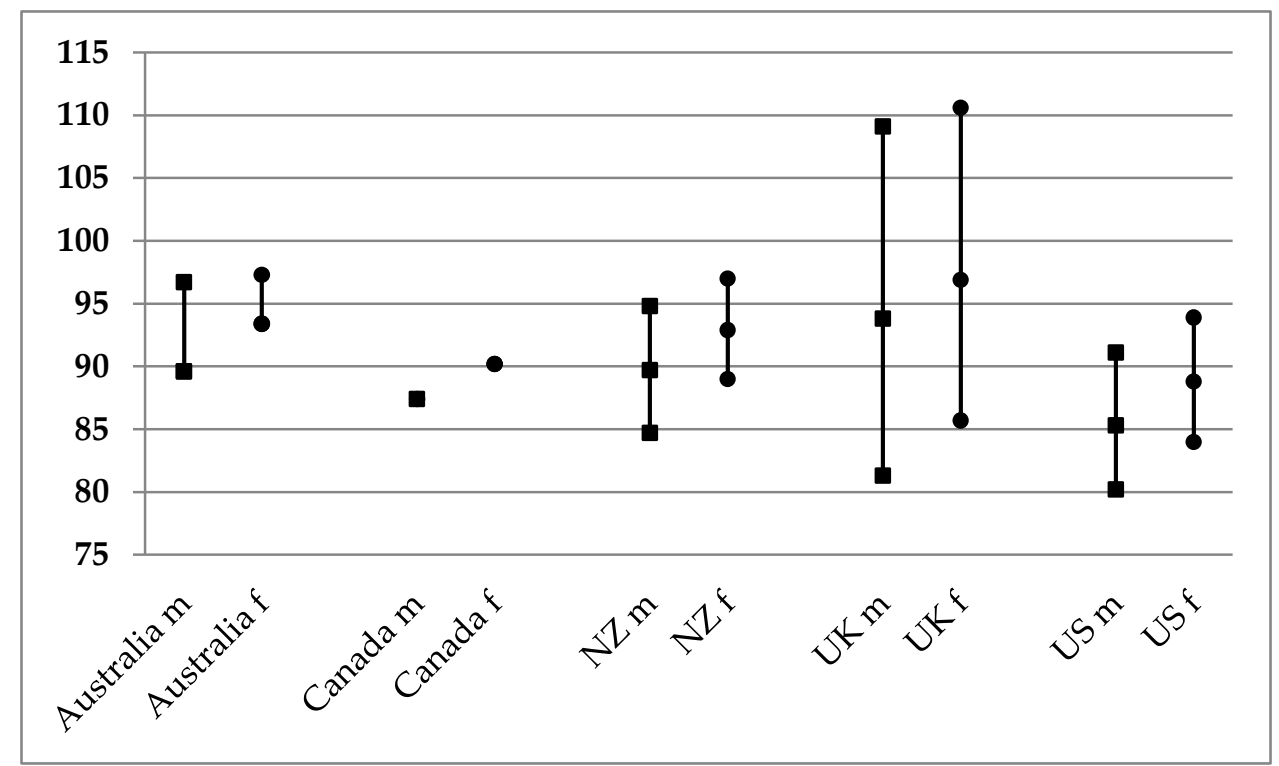

Source: See Table 5.3. 
Figure 5.3: Cohort life expectancy at age 65 in calendar year 2010 for selected countries, main projection variants

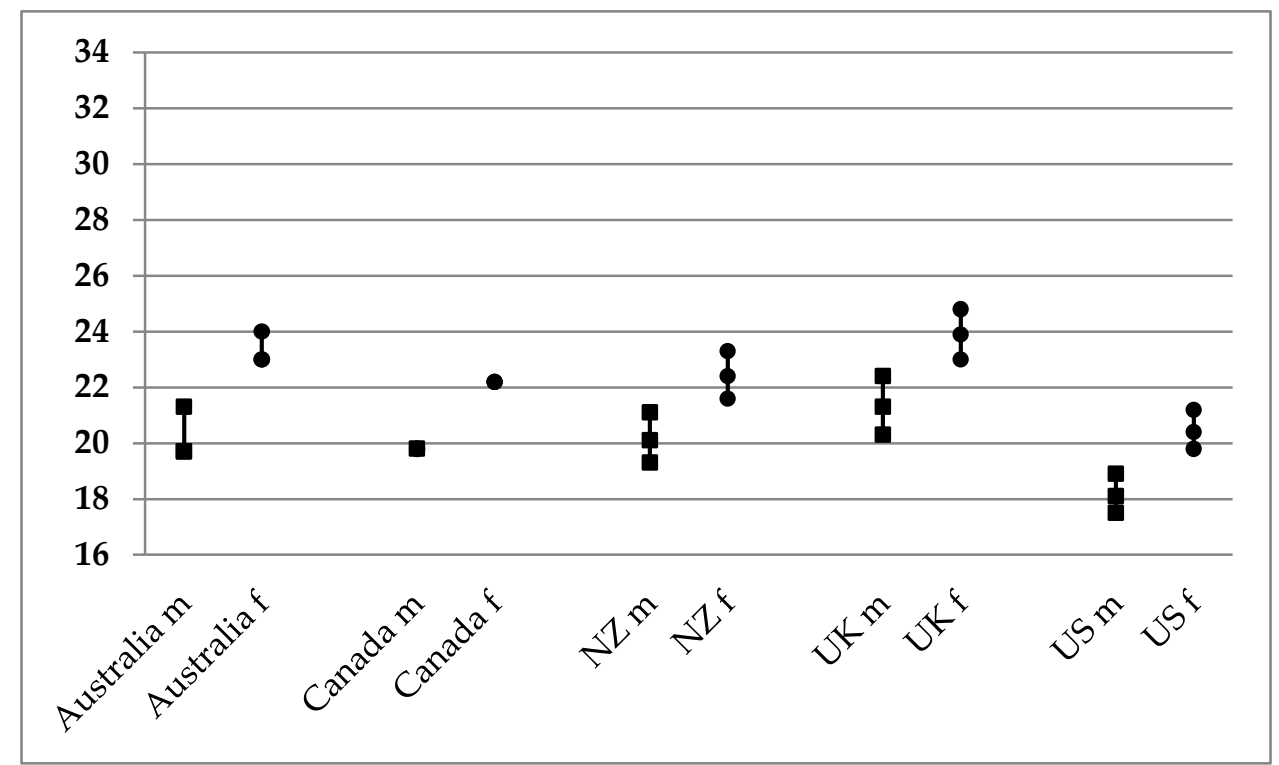

Source: See Table 5.3.

Figure 5.4: Cohort life expectancy at age 65 in calendar year 2050 for selected countries, main projection variants

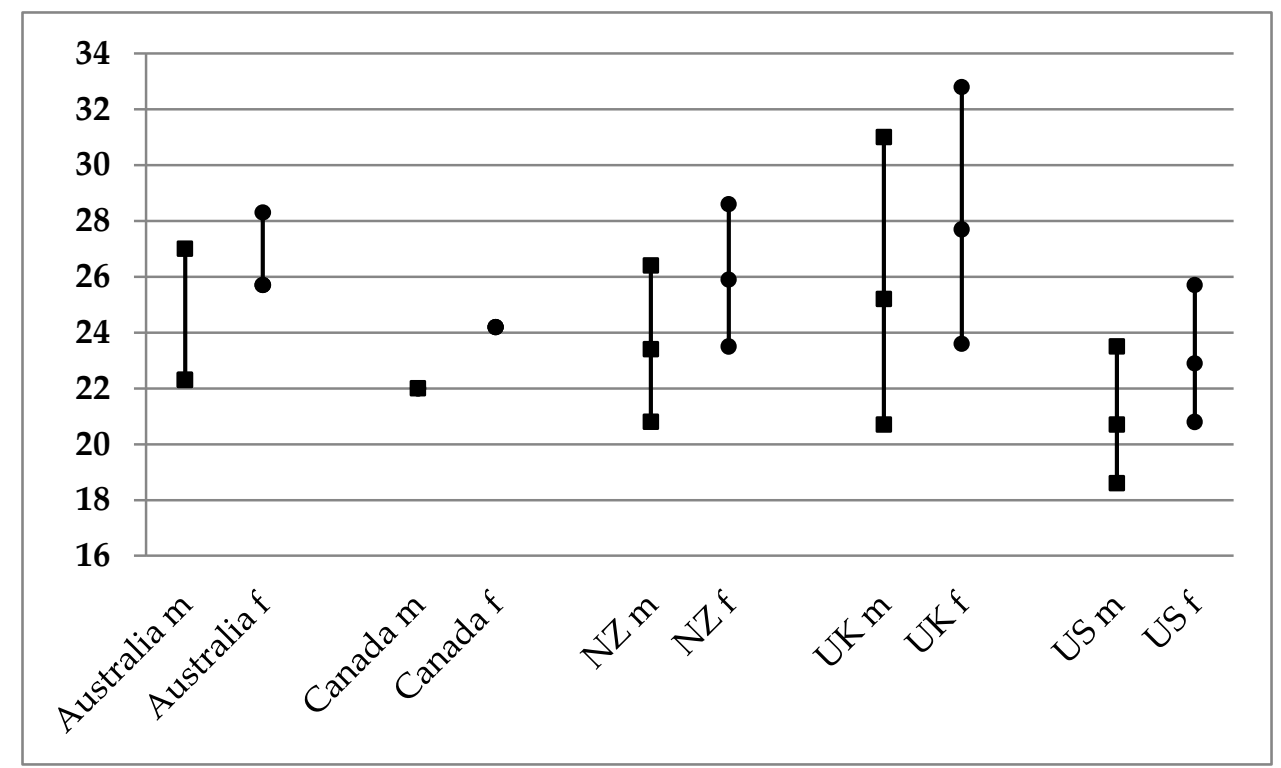

Source: See Table 5.3. 
The ranges of projected cohort life expectancy within each country (except where not available for Canada) illustrate the level of uncertainty about the future course of longevity. The UK shows the largest range, and the most optimistic projections for both lifespan at birth and at age 65. The higher variant for the UK appears more optimistic compared to that of other countries than the lower variant appears more pessimistic. This does not mean that the UK is more likely to achieve better lifespans than the other countries, or that there is any more uncertainty about longevity in the UK, but is a function of the largely arbitrary decisions on where to place the high and low variants.

There are large differences in projected average lifespan across countries, even for the 2010 cohort measures. For example, the expected average lifespan for boys born in 2010 ranges from 82 years in the US to 89 years in the UK, with the more optimistic of the two Australian projections at 92 years. The 2050 measures show an even larger range, so that the boundaries for all the variants across countries imply average lifespan for the cohort born in 2050 from 80.2 years for US males and 110.6 years for UK females. Remaining lifespan for those aged 65 in 2050 ranges from 18.6 years to 32.8 years (for the same groups).

The wide range in cohort lifespan measures reflects increasing uncertainty as assumptions of future mortality are made over the projection period, and the compounding of the relative differences in assumptions between countries. However, the projected future cohort measures underline again that all of these projections assume that mortality will continue to improve and that females will continue to live longer than males on average. Because of the assumption of continued mortality improvement, the cohort life expectancies 
are higher than relevant period life expectancies. Use of the period life expectancies will imply lower expected lifespans than the cohort life expectancies more realistically portray. Table 5.5 shows the difference between period and cohort measures, taking the projection period measures in Table 5.3 as the benchmark, therefore based on the medium projection for all except Australia.

Table 5.5: Excess of cohort life expectancy over period life expectancy at birth and at age 65 for years 2010 and 2050 from medium mortality projections in selected countries, in years

\begin{tabular}{|c|c|c|c|c|c|c|c|c|}
\hline & \multicolumn{4}{|l|}{2010} & \multicolumn{4}{|l|}{2050} \\
\hline & \multicolumn{2}{|l|}{ Male } & \multicolumn{2}{|c|}{ Female } & \multicolumn{2}{|l|}{ Male } & \multicolumn{2}{|c|}{ Female } \\
\hline & $\begin{array}{c}\text { At } \\
\text { birth }\end{array}$ & $\begin{array}{c}\text { At } \\
\text { age } \\
65\end{array}$ & $\begin{array}{c}\text { At } \\
\text { birth }\end{array}$ & $\begin{array}{c}\text { At } \\
\text { age } \\
65 \\
\end{array}$ & $\begin{array}{c}\text { At } \\
\text { birth }\end{array}$ & $\begin{array}{c}\text { At } \\
\text { age } \\
65 \\
\end{array}$ & $\begin{array}{c}\text { At } \\
\text { birth }\end{array}$ & $\begin{array}{c}\text { At } \\
\text { age } \\
65\end{array}$ \\
\hline Australia & & & & & & & & \\
\hline High & 12.3 & 2.1 & 9.5 & 1.9 & 8.4 & 2.0 & 6.8 & 1.6 \\
\hline Low & 6.5 & 1.1 & 6.3 & 1.1 & 5.6 & 0.8 & 5.1 & 1.0 \\
\hline $\begin{array}{l}\text { Canada } \\
\mathrm{New}\end{array}$ & 5.6 & 1.1 & 4.7 & 0.8 & 4.4 & 0.7 & 4.2 & 0.8 \\
\hline Zealand & 8.0 & 1.5 & 8.1 & 1.6 & 5.3 & 1.2 & 5.4 & 1.5 \\
\hline UK & 10.1 & 2.8 & 9.8 & 3.1 & 8.8 & 1.7 & 8.4 & 1.9 \\
\hline US & 6.6 & 0.8 & 5.6 & 0.7 & 5.0 & 0.8 & 4.8 & 0.9 \\
\hline
\end{tabular}

Notes and sources: See Table 5.3.

Table 5.5 shows that using period life expectancy to suggest average population lifespan would underestimate significantly from the more realistic figure given by the medium projection of cohort life expectancy. The underestimation reduces as the projection period increases as the period measure contains more estimation of future mortality improvement, so becomes more like the cohort measure. Across the countries, the potential underestimation from any confusion between period and cohort measures 
ranges from 4.7 to 10.1 years for the cohort born in 2010; and is a still important 1 to 2 years for remaining average lifespan for people aged 65 in 2050. This underlines the inherent potential for underestimation of lifespans using period measures instead of cohort measures of life expectancy. Underestimation could also come from using a medium projection when the high variant is more plausible, as the data for Australian variant projections in Table 5.5 suggest.

The increase in cohort life expectancy from 2010 to 2050 is less than that for period life expectancy; again because cohort measures in 2010 contain relatively more allowance for improving mortality than period measures. Table 5.6 shows the equivalent of Table 5.4 for cohort measures. The projected increases are 1 to 2 years less for cohort life expectancy at birth compared to period life expectancy at birth and generally less than half a year less for remaining lifespan at age 65 on a cohort basis compared to a period basis. Therefore, when increases in period life expectancy over 2010 to 2050 are presented, they tend to overestimate the extent of realistic change in average lifespans, albeit starting from an unrealistically low base. This may mitigate to some extent the underestimation inherent in period life expectancy measures, but the level of underestimation in expected lifespan compared to using cohort life expectancy is still more significant. 
Table 5.6: Increases in cohort life expectancy at birth and at age 65 from 2010 to 2050 from selected mortality projections, males and females, in years

\begin{tabular}{|l|cc|cc|}
\hline & Male & & Female & \\
\cline { 2 - 5 } & $\begin{array}{c}\text { At } \\
\text { birth }\end{array}$ & $\begin{array}{c}\text { At } \\
\text { age } \\
\mathbf{6 5}\end{array}$ & $\begin{array}{c}\text { At } \\
\text { birth }\end{array}$ & $\begin{array}{c}\text { At } \\
\text { age } \\
\mathbf{6 5}\end{array}$ \\
\hline Australia & & & & \\
High & 4.3 & 5.7 & 3.4 & 4.3 \\
Low & 3.6 & 2.6 & 2.9 & 2.7 \\
Canada & 2.6 & 2.2 & 2.3 & 2.0 \\
New & & & & \\
Zealand & 2.9 & 3.3 & 2.3 & 3.5 \\
UK & 5.0 & 3.9 & 4.5 & 3.8 \\
US & 2.9 & 2.6 & 2.8 & 2.5 \\
\hline
\end{tabular}

Notes and sources: See Table 5.3.

To complete the comparison of mortality projections between these selected countries, trends in past and projected future cohort life expectancies are now examined. This takes further the assessment of the implications for the choices of assumptions considered in Table 5.2, and illustrates that the divergence of period measures shown in Table 5.3 also holds for cohort life expectancy measures. For this comparison, England and Wales (E\&W) is used instead of the UK. England and Wales has a longer history of cohort data and the relevant available measures differ very little from those of the UK.

Australia is not included in this analysis as there are few available data points for the years selected, and no medium projection which for ease of presentation is the only variant shown in the following. The figures following show cohort life expectancy at birth for cohorts 1930 to 2050, first at birth and then at age 65. 
Figure 5.5: Cohort life expectancy at birth in years, by year of birth 1930 to 2050, for selected countries, medium projections, males

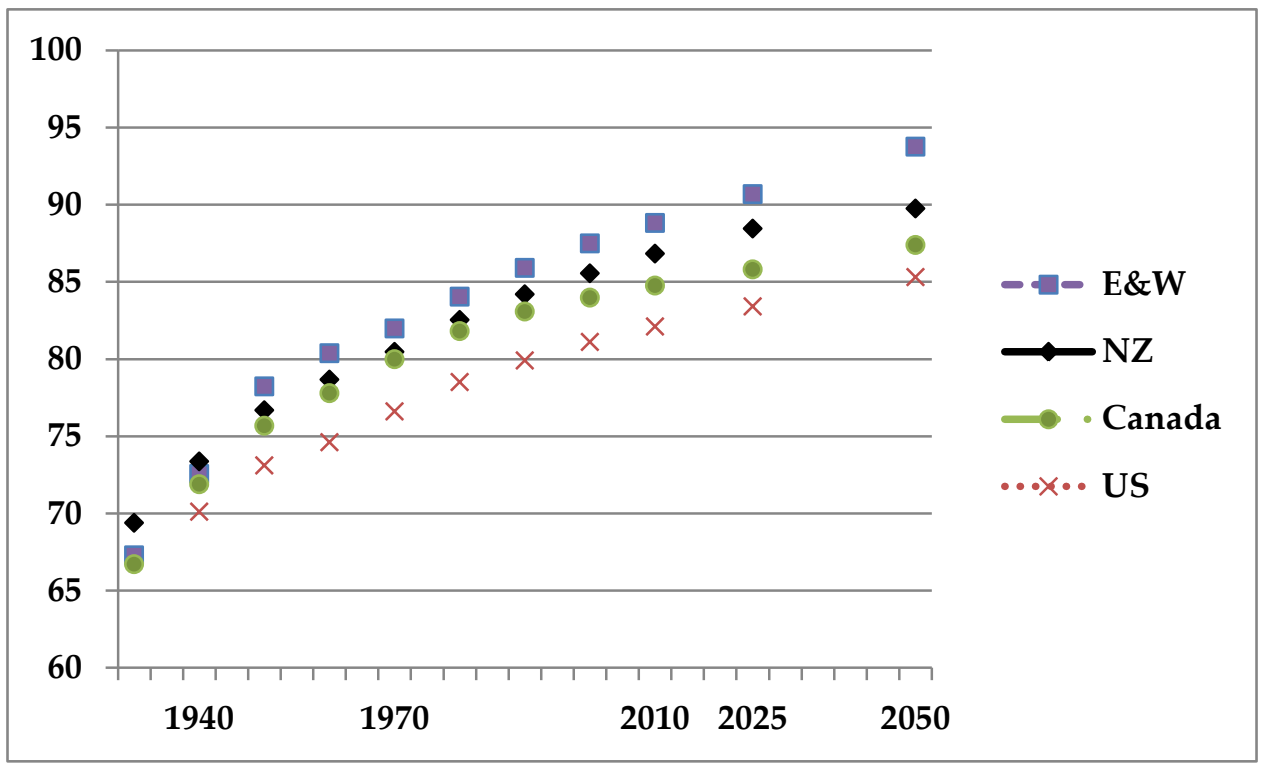

Notes and sources: See Table 5.3. Additional England and Wales data in personal communication from ONS.

Figure 5.6: Cohort life expectancy at birth in years, by year of birth 1930 to 2050, for selected countries, medium projections, females

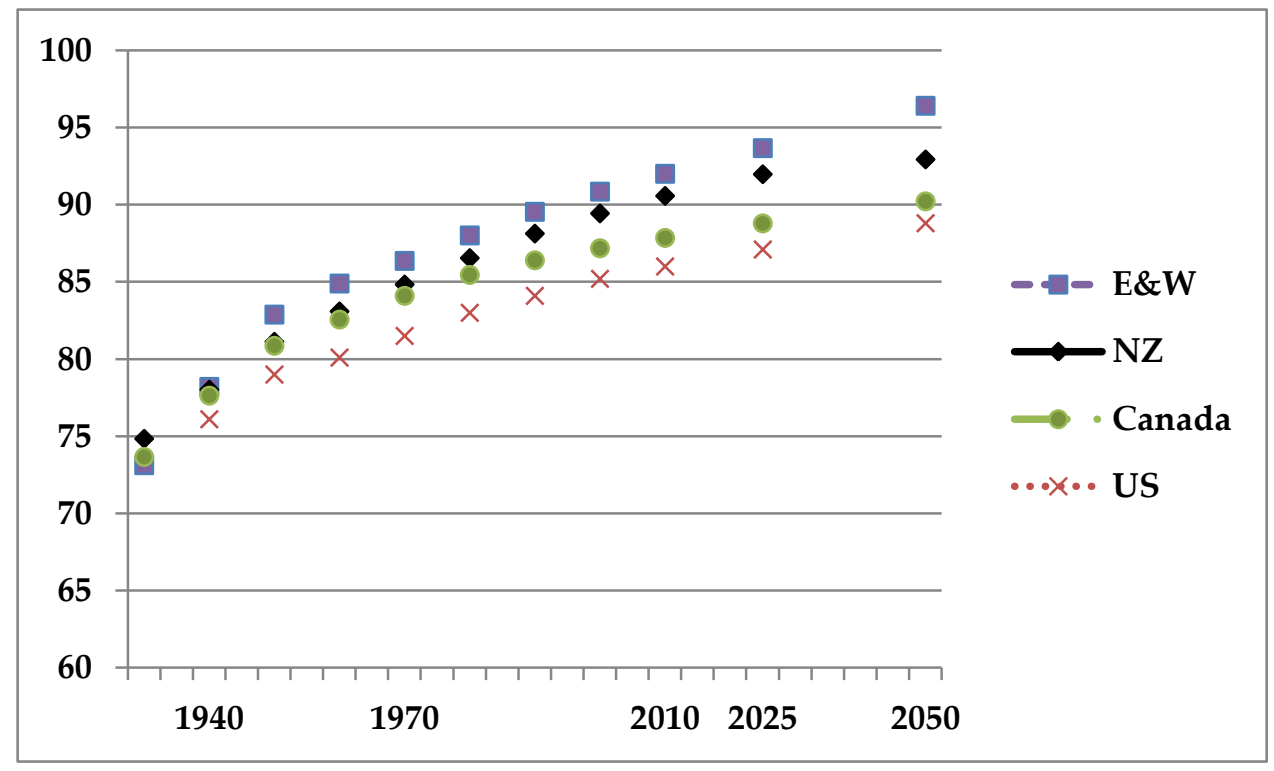

Notes and sources: See Figure 5.5. 
Figure 5.7: Cohort life expectancy at age 65 in years, by year of birth 1930 to 2050, for selected countries, medium projections, males

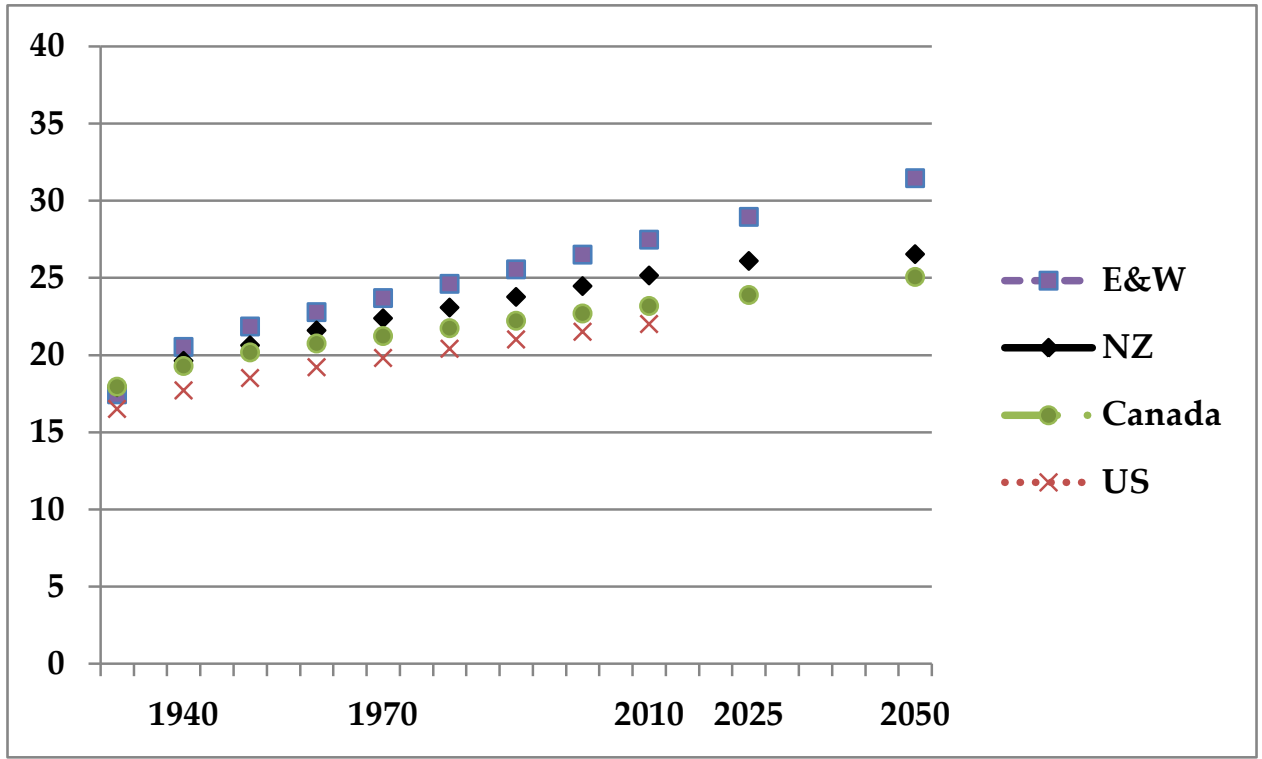

Notes and sources: See Figure 5.5.

Figure 5.8: Cohort life expectancy at age 65 in years, by year of birth 1930 to 2050, for selected countries, medium projections, females

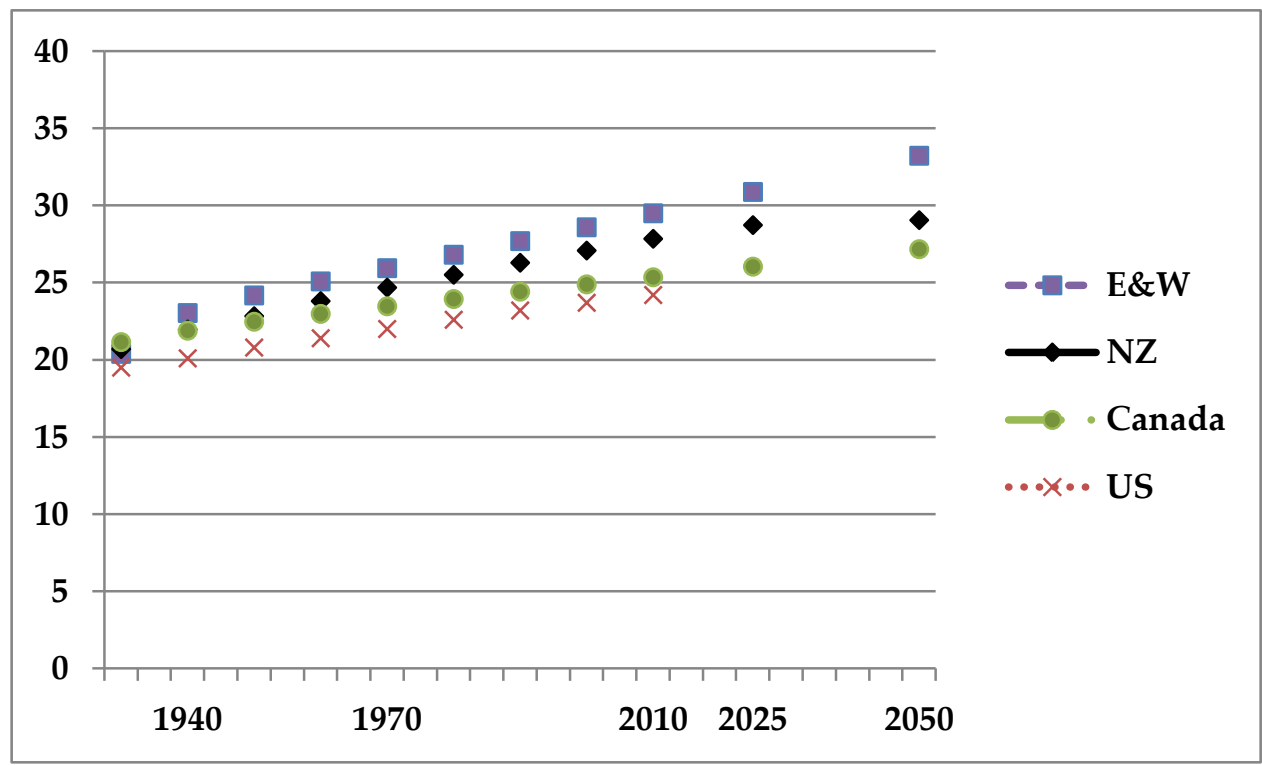

Notes and sources: See Figure 5.5. 
The early cohort measures in Figure 5.5 to Figure 5.8 contain more actual mortality data and fewer projected estimates than the later measures. The data extends for a longer period than previously shown estimates, as Figure 5.7 and Figure 5.8 are estimated remaining lifespans for people born in 2050, reaching age 65 in 2115. The figures show fairly close expectations of life across the selected countries up to around birth cohort 1970, but then the projections diverge. The divergence is not unexpected given the very long time frames over which the differences between assumptions compound. However, there is no theory or empirical evidence to suggest that over such a long period, the actual experience would be anything other than the cohort life expectancy of the countries continuing on similar tracks. The divergence of medium projections suggests a mutually inconsistent set of assumptions across countries. Insofar as the variant projections are intended to provide a boundary of potential outcomes, then continued close expectations of life across countries are possible, as Figure 5.1 to Figure 5.4 show. However, the medium projections are the estimates most often presented or referenced, usually exclusively. Further, the variant projections are an arbitrarily determined set of what is possible. This analysis emphasises that each of these singular pathways for the possible future of longevity in each country has to be understood as one potential scenario, based on only one suggestion for what past trends imply.

The position of New Zealand in the cohort life expectancy projections of Figure 5.5 to Figure 5.8 changes over time relative to that of England and Wales in particular. From the 1930 birth cohort New Zealand cohort life expectancies are greater than the E\&W equivalents. From 1950, the E\&W estimates exceed those of New Zealand and continue with an increasing gap of 
subsequent cohorts. This result is consistent with the changing position of UK and New Zealand period life expectancy projected over calendar years in Table 5.3, but the use of cohort life expectancies for successive birth cohorts reveals it happening sooner. This impression is a result of cohort life expectancies anticipating assumed mortality improvements.

This analysis adds to the comparison of past and future cohort life expectancies between England and Wales and New Zealand in O'Connell and Dunstan (2009). This study compared cohort life expectancies at birth and at ages 45 and 65 for the two countries for cohorts born in the years from 1876 to 1991. It identified that New Zealand had consistently higher life expectancies at birth than E\&W for both males and females for every cohort born to 1937. Cohort life expectancies at ages 45 and 65 were about the same for the two countries, with a small advantage to New Zealand again until the late 1930s. However, this remarkably consistent pattern, over a long time frame and for both sexes, was not expected to continue according to the projections of the statistical agencies. Later cohort life expectancies at all the ages analysed consisting of an increasing proportion of future mortality estimates over actual mortality data - diverged, with E\&W having the longevity advantage.

O'Connell and Dunstan pointed out that there was no obvious reason why this divergence should happen, especially as recent mortality improvement had been faster in New Zealand than in E\&W.

The comparison of historic QXI in Chapter 4 of this paper shows that New Zealand's recent mortality improvements have continued to outshine those of England and Wales, and high improvement rates have been spread over a wider range of ages. This analysis uses a later set of New Zealand projections 
that that available for the earlier paper: 2009-base instead of 2006-base. For the 2009-base projections, Statistics New Zealand changed its projection method and assumed more optimistic future mortality improvement than in the 2006base (Statistics New Zealand 2009a). The degree to which a gap opens up between projected cohort life expectancies between the two countries is attenuated. However, as the analysis of this chapter shows, the different assumptions made for the two medium mortality projections result in longer estimated lifespans on average in the UK than in New Zealand. Further, the variants presented suggest a range of outcomes for longevity that are balanced more towards optimism in the UK than is the case in New Zealand.

This analysis of past and future cohort life expectancies also adds estimates from Canada and the US to compare with that from New Zealand and England and Wales. This additional data confirms the general shape of improvements expected in average lifespans for successive birth cohorts. All of these countries anticipate ever longer average lifespans, with the shape of the curves in Figure 5.5 to Figure 5.8 being very similar across countries. However, the ranking of future cohort life expectancies projected for the four countries appears somewhat inconsistent. Not only does England and Wales look relatively optimistic, but Canada's projections suggest undue pessimism, given the strong mortality improvement seen in Canada. The projections in Canada assume the rate of mortality improvement slows down to a level less than half what Canada has actually experienced in the last forty-five years, although there is no rationale for why this should be so. The projections for the US keep the US at the bottom of the ranking for life expectancy in this group of countries and this appears likely in the absence of any different policies for change. 


\subsection{Reflecting New Zealand's relative mortality experience}

The comparative international analysis in Chapter 4 showed that, despite many similarities in the level of and trends in mortality rates, New Zealand is notable for its fast rate of mortality improvement at ages over 40 and especially over 80; and for its relatively high mortality at ages under 20 with high recent mortality improvement at ages 15-25. Further, while the UK has had the closest level and average improvement rate of mortality compared with New Zealand, in the last two or three decades New Zealand's rates of mortality improvement have been more like Australia's. This section takes these notable features of New Zealand's relative mortality experience and discusses how this might influence the interpretation of the relative position of New Zealand's mortality projections. The nomenclature for Statistics New Zealand variant projections now switches back to Low, Medium and High Mortality rather than the low, or high life expectancy labels used for international comparisons in Table 5.1.

\section{Relatively strong momentum in mortality improvement}

In absolute terms, and compared to its peers, New Zealand has had particularly strong improvement in mortality in the last twenty-five years. The New Zealand total population annual rate of change in mortality rates (QXI) gained momentum throughout the forty-five year period of the comparative analysis, with female mortality improvement accelerating in the 1980s before that of males in the 1990s (Table 4.4). Because future mortality projections are estimated by extrapolating forward the past mortality trend, the changing pace of New Zealand's QXI is intriguing. If the last twenty-five 
years of mortality trend were judged to provide the basis for New Zealand's future trend, then projections would result in higher projected lifespans than if the past forty-five years were used. On this basis, the Low Mortality variant of Statistics New Zealand mortality projection appears to be a useful benchmark for a plausible likely future for New Zealand mortality.

The average rate of mortality improvement over the last twenty-five years in New Zealand was of the order of the average QXI assumption for the Low Mortality variant (see the high life expectancy variant of Table 5.1). Moreover, the Australian projections are carried out using QXI from the past 100 years and the past twenty-five years. The former still uses QXI higher than New Zealand's medium projection, with the latter QXI similar to those in Low Mortality. Using Australia as a role model would therefore reinforce Low Mortality as plausible. Finally, the UK's similar past QXI also points to using future QXI assumptions more like the UK's which differ in shape: the UK projections favour early high QXI which then flatten out. Low Mortality gives similar projections of cohort life expectancy to the medium projections for the UK (Figure 5.1 to Figure 5.4).

Low Mortality uses a lower QXI for men compared to women whereas the reverse is true for Australia. Past QXI for New Zealand men were lower than for women in the 1980s, although male mortality improvement recovered some ground in the next decade. In the UK, QXI assumptions are similar by gender, although the 1980s were similarly relatively poor for men. The 1980s were an unusual decade for New Zealand, in that variance in age at death for men increased markedly (see section 6.3). Therefore, there is an argument for male mortality in New Zealand to continue at a rate of improvement similar to 
that for females in future, which would add to future QXI being overall higher than the medium variant and more like the overall effect of Low Mortality.

New Zealand's more recent rate of mortality improvement was relatively strong across all ages, particularly so over age 40 , and with a putative golden cohort emerging for birth years 1967-71 for males and 1967-76 for females. The strong mortality improvement at older ages is significant as in countries like New Zealand which some way on the path of mortality compression, the change in life expectancy measures increasingly comes from mortality improvement at older ages. Therefore, the momentum in mortality improvement in New Zealand is operating at the ages where it can have most effect on lifespan measures. The emerging golden cohort at younger ages is important as it suggests that New Zealand is better positioned for future mortality improvement than the UK, which is the only country among those analysed not to shows signs of a young golden cohort. Thus, the age profile of mortality improvement in New Zealand gives reasons to be optimistic about future mortality improvement and relatively optimistic compared to the UK, which itself makes optimistic assumptions about future continued mortality improvement. Again, this implies a plausible future of mortality in New Zealand which is better than the Medium Mortality and more like the Low Mortality projection.

\section{Relatively poor mortality for children and young adults}

Although New Zealand generally has favourable mortality compared to comparator countries, its mortality for children and young adults appears relatively poor in the results of Chapter 4's analysis. This section briefly sets some international context for what is known about mortality at these ages, 
before considering how New Zealand's mortality profile at young ages may change in future.

The level of childhood mortality is poor in all the British settler countries considered in this thesis relative to other developed countries. The five selected countries had the highest probability of dying between birth and age 5 (the under five mortality rate) in the OECD in 2001-4: 6.0 per 1,000 live births for New Zealand and Australia, 6.5 for Canada and the UK and 8.0 for the US. The ranking of these countries on this measure has worsened since the 1960s (Collinson et al. 2007). Globally, research and policy attention has focused more on childhood mortality than mortality in adolescents and young adults. However, in most countries over the fifty years to 2004 adolescent (ages 15-24) death rates have not fallen as much as those for children. This is because mortality rates from accidents have remained static or risen and violence and suicide have increased while the rate of mortality from diseases affecting childhood mortality has reduced (Viner et al. 2011). In New Zealand, across ages 1 to 19 , nearly 60 per cent of deaths result from external causes, especially transport and other accidents. In the 15-19 age group, the proportion is 74 per cent as the rate of involvement in traffic accidents increases and intentional self-harm appears as a cause of death (Ministry of Health 2010 Tables 2 and 3).

Mortality rates at ages 1-20 are very low in developed countries including those considered in this thesis. In 2010, there were 284 deaths in New Zealand of New Zealand residents aged between 1 and 19 inclusive (Statistics New Zealand data). The probability of living from age 1 to age 20 is 99.3 per cent for males, and 99.6 per cent for females (Statistics New Zealand 2009c). The analysis in Chapter 4 used mortality rates from life tables where the data were 
smoothed. It is possible comparison of actual death rate data even in the same years would attenuate the significance of differences between countries. Further, the low current mortality rates at young ages mean that assumed reductions in future would make little difference to the overall projections of population average mortality. For example, period life expectancy at birth for New Zealand males is projected to increase by 5.6 years from 2010 to 2050 (as per Table 5.4). In that projection, an assumption will have been made for continuing mortality rate improvement at ages 1-19 in the order of past improvement rates between 1 to 3 per cent a year (Table 4.5). An unanticipated further improvement in mortality rates at that age of twenty per cent would result in a further increase in period life expectancy at birth of around 0.09 years (calculated using 2005-7 life tables from Statistics New Zealand 2009c). Therefore, the apparent notable poor mortality in New Zealand at ages 1-20 should not be a reason to adjust existing projections of population mortality.

\subsection{Impact of potential adverse trends}

A common pessimistic theme in mortality forecasting is the increasing prevalence of obesity and the mortality risk from its sequelae. Olshansky et al (2005) suggested that obesity may cause a halt or reversal to the rise in life expectancy in the US. Stewart et al (2009), again for the US, forecast that obesity trends could hold back the increases in life expectancy brought by declines in smoking.

These forecasts have been criticised. Preston (2005) listed objections to the reasoning in the Olshansky article that apply equally to the methods used by Stewart et al. The studies did not allow for medical improvements or 
behavioural changes to mitigate the mortality risk from obesity; for the fact that forecasting by extrapolation already includes an implicit assumption that the mortality effect from the past increase in prevalence of obesity continues; or for mortality from non-obesity related conditions to continue to improve. These points were reiterated in a later review of US mortality (Crimmins et al. 2010b), which further suggested that the mortality risk associated with obesity may have declined as the increase in obesity prevalence has levelled off, although a cohort of obese may be working through. Incorporating more data on smoking, obesity and other mortality trends than the previous forecasts, King and Soneji (2011) forecast that declines in smoking would outweigh the effect of obesity so that US life expectancy would continue to rise throughout their projection period to 2030. Compared with the Olshansky and Stewart predictions, this forecast is more in line with knowledge that life expectancy has only ever declined when countries have experienced specific severe direct assaults on mortality such as the significant HIV/AIDS epidemic in parts of Africa or the change in social order in post-Soviet states (Wilson 2011).

Knowledge of the effect of obesity on future lifespans is not complete. There appears to be a "J-shaped" relation between Body Mass Index (BMI) and mortality from cardiovascular disease, some cancers and all causes. Most of the cardiovascular mortality risk is caused not by high BMI itself but is mediated by other associated risk factors: diabetes, elevated blood pressure and elevated serum cholesterol (Huxley and Jacobs Jr 2011). Data on the prevalence of these four risk factors from 1980 to 2008 worldwide is analysed here to put New Zealand's risk position in context with those of the other countries of this analysis. Figure 5.9 and following show the data for the latest year available. 
Figure 5.9: Age-standardised mean BMI in $\mathrm{kg} / \mathrm{m}^{2}$ by country in 2008 , with 95\% uncertainty intervals, male (left) and female (right)

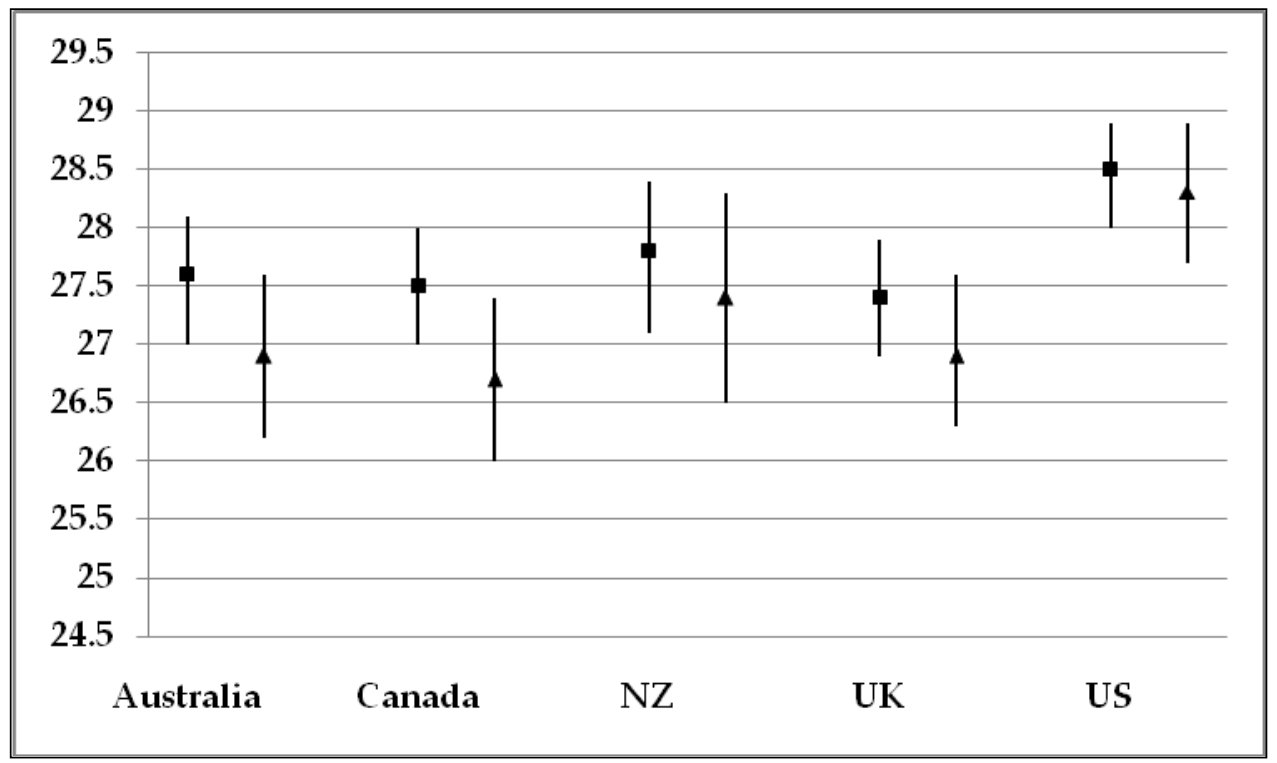

Source: Finucane et al (2011).

Figure 5.10: Age-standardised diabetes prevalence by country in 2008, with 95\% uncertainty intervals, male (1) and female (r)

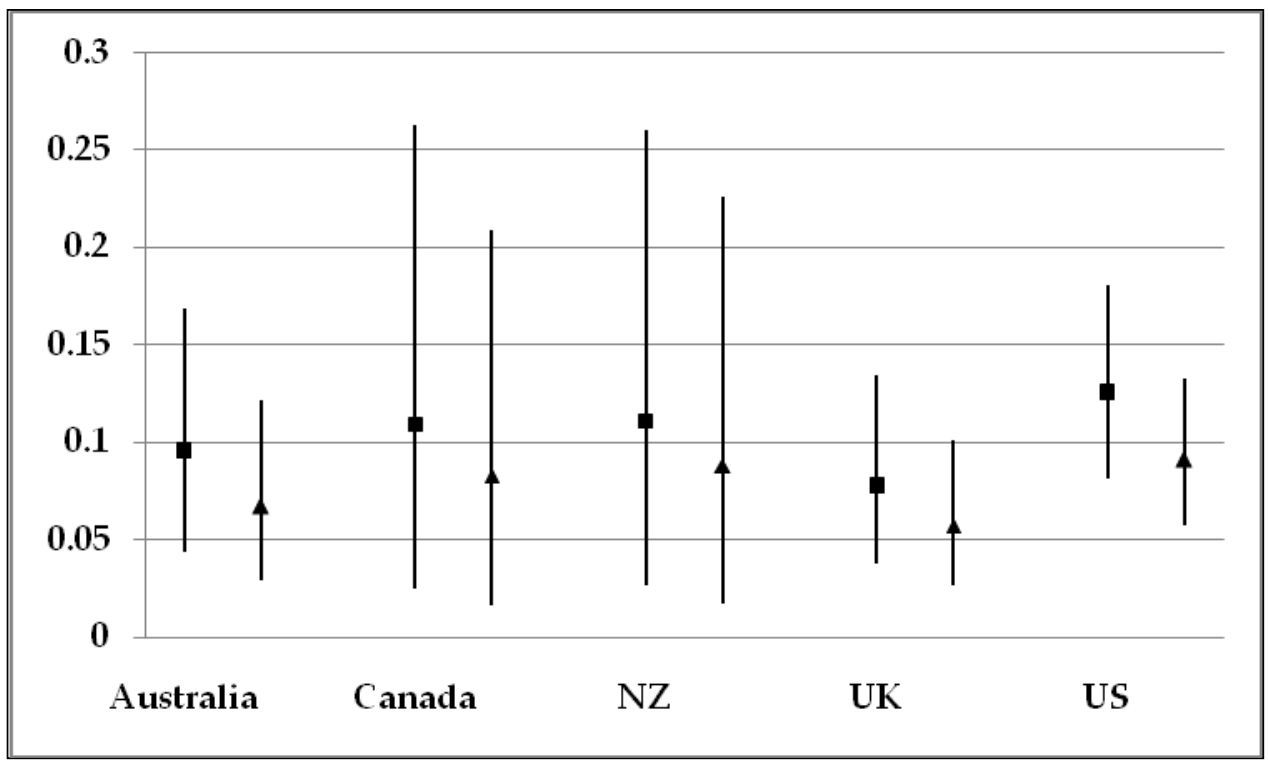

Source: Danaei et al (2011b). 
Figure 5.11: Age-standardised mean systolic blood pressure in $\mathrm{mmHg}$ by country in 2008 , with $95 \%$ uncertainty intervals, male (l) and female (r)

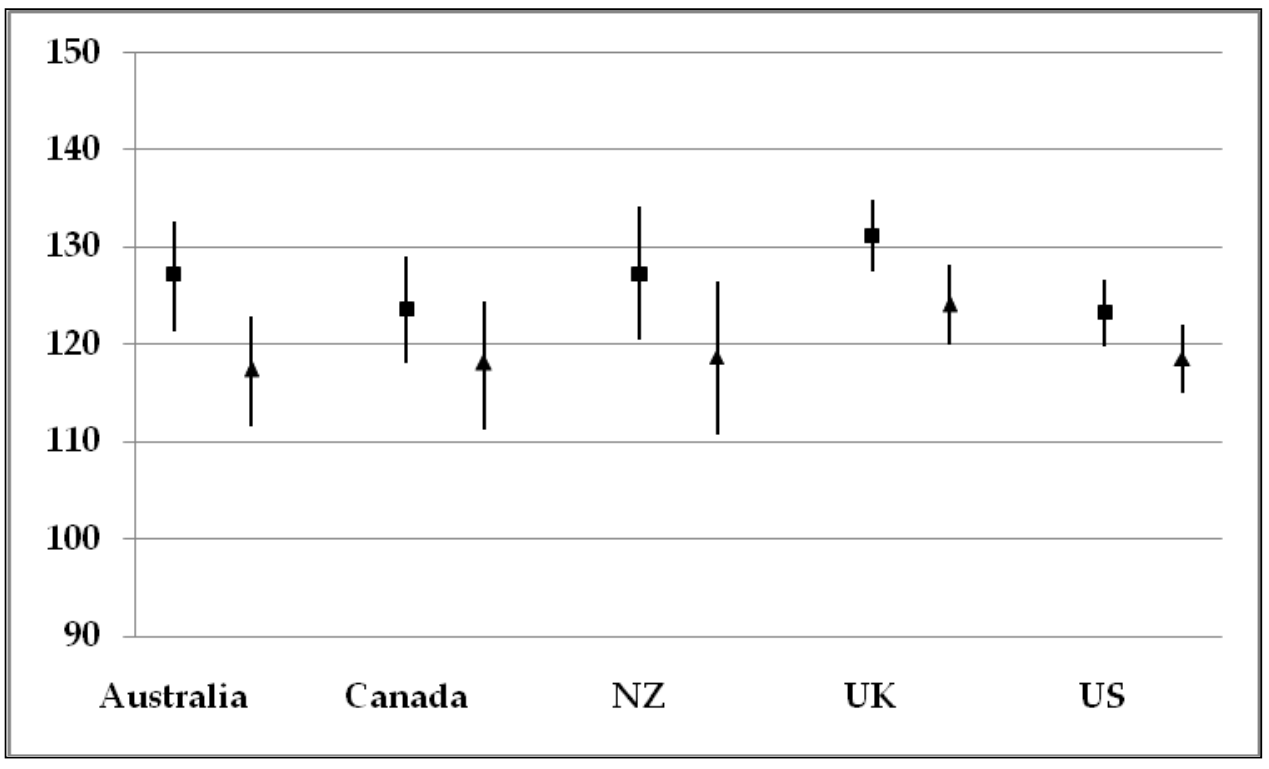

Source: Danaei et al (2011a).

Figure 5.12: Age-standardised mean serum total cholesterol in $\mathrm{mmol} / \mathrm{L}$ by country in 2008 , with $95 \%$ uncertainty intervals, male (1) and female (r)

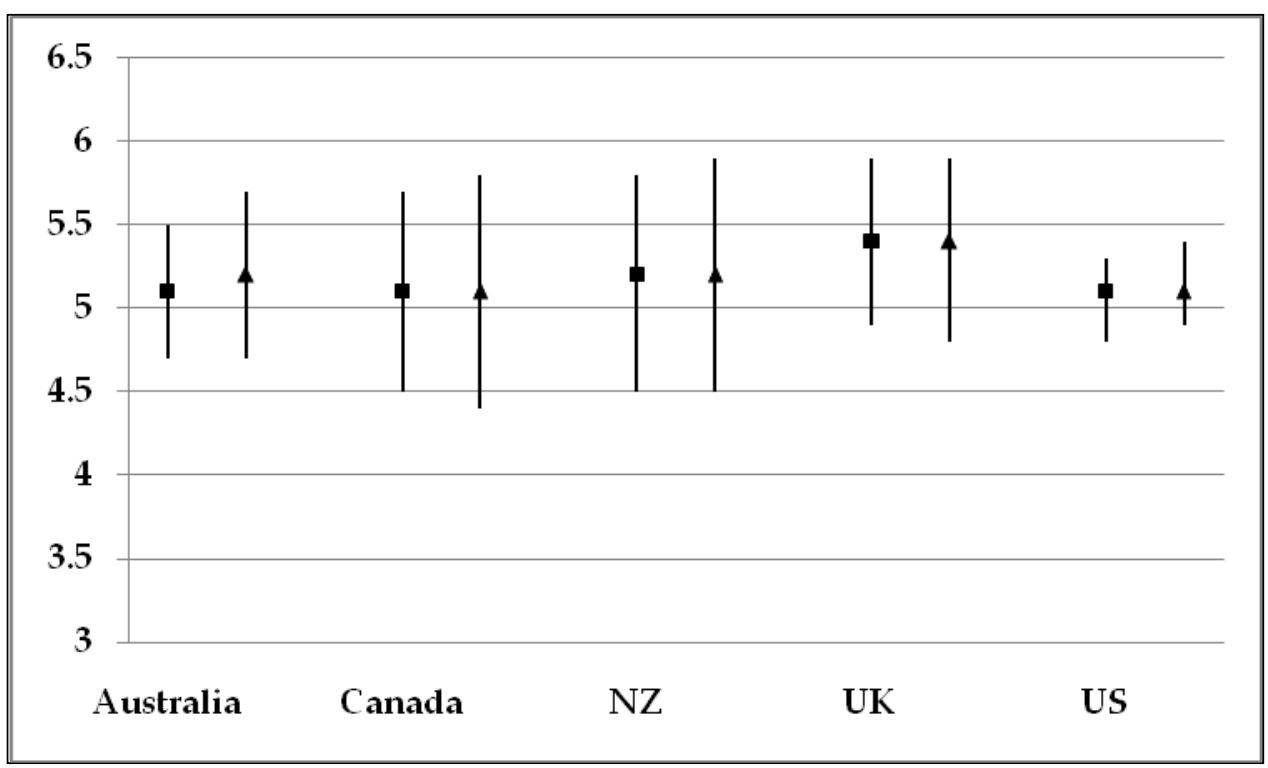

Source: Farzadfar et al (2011). 
The US has one of the highest age-standardised mean BMI in the world, with few countries in the Pacific and Middle East having higher. Over the period of the available data (1980 to 2008), mean BMI has increased globally. Mean BMI is within the range of 'overweight' (BMI $25-29.9 \mathrm{~kg} / \mathrm{m}^{2}$ ) rather than 'obese' $\left(>=30 \mathrm{~kg} / \mathrm{m}^{2}\right)$ in all five countries considered in this analysis. Of these, New Zealand has the second highest mean BMI, but is closer to the lower levels of the others than to the outlier of the US. Only Canada has had a lower increase in BMI for males, although for females the New Zealand increase is the same as that in the US, with only Australia higher.

Diabetes prevalence increased globally from 1980 to 2008 . The Pacific region has the highest levels. As with BMI, New Zealand is second to the US of these five countries for prevalence and increase for females, but third after Australia for increase for males. A different picture prevails for blood pressure and cholesterol as the UK has the highest mean, with New Zealand second, and in all five countries the means are falling. This commentary summarises the relative positions of the means, but the uncertainty interval is large enough to change rankings, with a particularly large interval for diabetes.

There is therefore a mixed picture, with mean BMI and diabetes prevalence rising in New Zealand and other countries, while the other risk factors decline. Judging from the situation and momentum on these risk factors, New Zealand appears to have lower obesity-related risk potential than the US, and similar to Australia and the UK, the role models for mortality extrapolation. Although the UK has lower diabetes prevalence, it has higher 
mean blood pressure and cholesterol. In all countries an increase in mean BMI has been in past mortality data, so implicitly the impact on future mortality should be taken into account in extrapolation.

In New Zealand, Māori and Pacific people have a higher prevalence of obesity and diabetes compared to the total population. Asian people are less likely to be obese, but more likely to have diabetes than the population (Ministry of Health 2008). There is the possibility that diabetes risk changes as a result of future migration patterns. On average for the total population, agestandardised death rates from diabetes have gradually declined in New Zealand from the late 1990s (Ministry of Health 2010). Age-standardised obesity prevalence increased from 1997, but there was no statistically significant increase from 2002/03 to the latest figures in 2006/07 (Ministry of Health 2008). This may indicate that New Zealand is following the trend in the UK and US where detailed surveys suggest obesity prevalence has levelled off since 2000 (Wang et al. 2011).

Obesity-related mortality risk can be prevented or diminished by medical treatment and behavioural change (The Lancet 2011). Even small weight losses can make a significant difference to the risk outlook (Wang et al. 2011). US data suggests the mortality risk associated with Class I obesity (BMI between 30.0 and 34.9) appears to have reduced in the 1990s compared to earlier decades (Mehta and Chang 2011). Future mortality risk from obesity may therefore depend on the effectiveness of treatment and prevention. Health policy is being directed towards this goal because of the significant and increasing economic cost of obesity and related conditions. Based on the latest forecasts from the US, the comparator country with the highest risk from 
obesity and related conditions, and assuming New Zealand does participate in a global trend to reduce obesity prevalence and its effect on mortality, it is hard to see obesity as a major influence on New Zealand's projected population average mortality level or the relative position of its projected life expectancy.

\subsection{Conclusions}

The projections of New Zealand's future life expectancy are based on an extrapolation of past internal trends, as are those in the other countries selected for this analysis. All of these countries have experienced continued improvement in mortality rates, and mortality rates are assumed to continue to improve in future. No projection involves mortality worsening in future. This means that in all projections for the selected countries lifespans are assumed to continue to lengthen over time. The difference between projections of different countries is the assumed trajectory of future mortality improvement. This shared assumption of continued mortality improvement confirms that current or projected period life expectancies cannot be relevant future lifespan measures, and cohort life expectancies are preferred here to report on the results of the projections.

The possible causes of past mortality trends have been examined in great detail and the potential future path of mortality similarly discussed at length. Some of this qualitative theorising was covered in Chapter 2. Data for past mortality levels and trends and lifespan measures for the selected countries were presented in Chapter 4. Official projections of future mortality are undertaken with more data available for each country. This combination of detailed revealed and unrevealed data of the past and theorising about future 
trends gives the appearance of more accuracy in the projections that is actually present. The assumptions identified here that underlie each set of projections are generally very simple and somewhat arbitrary, particularly about decisions on the range of variant projections presented and the shape of mortality improvements assumed. All the projections are 'what-if' scenarios essentially assuming a simplified continuation of the historic average mortality trend.

The agencies making assumptions about the future path of mortality hold different implicit or explicit views on the trajectory of that path. Convergence or divergence in mortality levels between these countries has not been stated as considered explicitly by any of the agencies, although long-term convergence is a valid hypothesis given long-term past trends. It seems plausible that the US would retain the lowest ranking, as the causes of its relatively low mortality have been investigated with no reason why it should overtake other countries in mortality improvement suggested. However, there has been no reason identified for why the other countries should change relative position; why Canada should fall behind, why the UK should jump forward, and especially why New Zealand and the UK should diverge when current and past life expectancy measures have been close. The assumptions behind the projections provide a reason why they turn out this way, but not a rationale.

Instead, the relative performance of past mortality trends imply that New Zealand's official mortality projections seem relatively pessimistic. The assessment of New Zealand's recent relative mortality position in Chapter 4 gives several reasons why New Zealand's future mortality could be better than 
assumed in the main projection. New Zealand's fast mortality improvement in recent decades is more consistent with that of Australia which has more optimistic future mortality assumptions. Relative to the UK, notwithstanding the long history of similarity in mortality levels, divergence if it occurs would be likely to give the advantage to New Zealand which has a young cohort with high mortality improvement that the UK does not have. The UK projections appear relatively optimistic because high mortality improvements are assumed in the short term - a path which based on recent experience New Zealand may be better placed to follow. The projections in Canada appear pessimistic because they assume the rate of mortality improvement slows down to a level less than half what Canada has actually experienced in the last forty-five years, with no apparent rationale for why this should be so. By comparing the assumptions and outcomes of these relevant comparator projections, the Low Mortality variant of Statistics New Zealand's projections appears plausible.

Factors that made New Zealand a relatively healthy country a century ago - clean air, low population density and benign climate - are still present. In all countries considered here increasing obesity prevalence has been in past mortality data, so the impact on future mortality will have been taken into account in existing extrapolative forecasts. There is not sufficient evidence that New Zealand's mortality risk from obesity and related factors would be significantly higher than that of Australia or the UK to change the conclusion that these countries should be role models for mortality projections. Mortality improvements could slow if healthcare spending were reduced, or if health policy were not effective at treatments or modifying behaviour to reduce mortality risk from obesity and its sequelae and other causes of death. 
However, the threat of rising health costs from obesity-related problems would suggest policies to prevent and treat these risks will be increasingly adopted. Knowledge about how known risks such as obesity and smoking may play out in future is limited, as is knowledge about how other health risk or improvements may combine to affect population average mortality. Therefore, while negative factors exist, there is no evidence to contradict a conclusion that future longevity in New Zealand is likely to reach at least the Medium Mortality estimate of Statistics New Zealand's projections and, more plausibly from the comparative analysis presented here, to follow the Low Mortality variant. 


\section{Chapter 6: Mortality within New Zealand}

This thesis focuses on a plausible future for population average lifespans and how individual expectations and policy assumptions at the national population level differ from that. In projections of mortality by national agencies, variance in mortality or lifespan is not explicitly considered. However, mortality variation is an important subject for policy and theoretical reasons.

The third part of the mortality analysis framework developed in this thesis considers mortality within New Zealand. The questions asked are: given past mortality trends for the different sub-populations, does the projection of future total population mortality look plausible? Are there unusual trends in one sub-population which could disturb the extrapolation of mortality in the total population? Answers are sought to these questions by testing the mortality of subgroups within New Zealand against emerging demographic theories on mortality variance.

In this chapter, the demographic theory of mortality variance is first set in the context of the epidemiological approach to mortality inequalities. The second section reports on the contribution of mortality variance to the mortality analysis framework used here. The third section briefly compares mortality variance in New Zealand with that of the peer countries of this analysis. 


\subsection{Theories of mortality variation}

There is a large literature on the variation of life expectancy or mortality between and within countries in the fields of epidemiology and public health. The focus in these disciplines is on avoidable health inequalities, also referred to as inequities or disparities, in particular the association of inequalities with socio-economic position (Marmot 2005; WHO 2008). The theory of fundamental causes holds that those of higher socio-economic status accumulate resources that contribute to good health and longevity (Phelan et al. 2010).

The work on the socio-economic determinants of health is not without criticism (for example, Cutler et al. 2006; Epstein et al. 2009; Oliver and Nutbeam 2003). The theory of fundamental causes predicts women would have higher mortality and shorter lifespans than men on average, yet that is not the case in all but a few less developed countries. The extent to which national income inequality is directly associated to population health appears to vary markedly by country, if at all (Lynch et al. 2004). New evidence is still

adding to theory. For example, income inequality may be related to avoidable causes of death among children and working age adults but appears unrelated to life expectancy losses from the diseases of old age (Shkolnikov et al. 2011a). Causality between socio-economic indicators and health inequalities is not proven and it is unlikely that socio-economic factors provide a full explanation for mortality differences between or within countries. 
Within demography, the variance of mortality has a smaller literature, largely to do with the relationships between population average life expectancy, population average mortality rates and variance in age at death. This literature shows three relevant mathematical rules, which are explained below. First, that there is inherent variation in mortality or lifespan in a population. Second, that variation in lifespan is inversely correlated to levels of life expectancy, except at older ages. Third, that gains in life expectancy depend on how far a population is on the path to mortality compression.

\section{Inherent variation in mortality or lifespan}

The most enduring of attempts to theorise the shape of average mortality in a population is Gompertz Law which has been proven to hold for different populations and periods at all ages except the very youngest and oldest (Gompertz 1825; Olshansky and Carnes 1997). Gompertz Law posits that the force of mortality increases in geometric progression as age $\mathrm{x}$ increases arithmetically.

$$
\mu_{x}=k e^{\beta x}
$$

The force of mortality $\mu_{x}$ can be considered a further variant of a mortality rate, being almost consistent with the mortality rate at age half a year less except at the oldest ages. In the above equation, $\mathrm{k}$ is a constant and $\beta$ is the slope of the graph of the logarithm of the force of mortality drawn with age, sometimes called the rate of ageing. 
Tuljapurkar and Edwards (2011) show that the variance in age at death for a population following Gompertz law is a function only of the parameter $\beta$, and that the rate of ageing is inversely related to the variance in length of life.

$$
\text { Variance in age at death } \cong 1 / \beta^{2}
$$

Why this should be so, or what this means for future trends, is unclear. However, since $\beta$ is a finite number, and Gompertz Law applies for human populations at most ages, it does imply that there will be some non-zero variance in mortality in national statistics. This can be seen as a natural or inherent variance in age at death within a population, or a reminder that there is an element of chance in the way biological processes operate. Some variance in age at death will be present in all populations, and not all variance will be avoidable inequity.

\section{Variance in lifespan correlated to life expectancy}

Empirical evidence shows that variance in total length of life is negatively correlated to life expectancy, except for populations of older survivors. Smits and Monden (2009) demonstrated a high negative correlation between life expectancy at age 15 and absolute inequality in age at death from a database of 212 countries dating back at least to 1950 and for some countries to the 18th century. A long-term trend in most developed countries on the path to mortality compression has been that as life expectancy at birth has increased variance in age at death has decreased. A corollary important when comparing variance in longevity or mortality across countries is that differences in variance should be interpreted only between populations of the same life expectancy at birth (e) $\mathrm{e}_{0}$, so at similar points on the path to mortality compression. 
The inverse correlation between life expectancy and variance breaks down at older ages however. In low mortality countries, mortality compression appears to be tempered by mortality shifting, so that as the curves of deaths shifts to the right, the peak does not continue to narrow endlessly (Ouellette and Bourbeau 2011). Engelman et al (2010) examined the changing distribution of ages at death from 23 national populations over the last 5 decades, and found that while the variance in mortality reduced for the full population, and for survivors to age 10, the variance in longevity among survivors to older ages slightly increased. They attribute this to a shifting of health disparities from younger to older ages as a result of the success of early life mortality improvements. There is a wider range of frailty among older survivors in high life expectancy populations compared to lower life expectancy populations.

Thus for developed countries with the highest life expectancy, variation in total lifespan might reduce as the survival curve rectangularises while heterogeneity in age at death for the oldest survivors increases. This is possible even given the Tuljapurkar and Edwards equation as Gompertz Law breaks down at older ages, and the equation needs to be modified. Countries further on the path to compression of mortality at older ages would be expected to have greater variance in age at death among older survivors.

\section{Gains in life expectancy depend on path to mortality compression}

The definition of mortality inequality in a population is that too many people die too young. In a high life expectancy population well advanced along the path to mortality compression, deaths are concentrated at ages around a 
narrow mode. There is less variance in age at death than a low life expectancy population with more deaths at younger ages and a less rectangular survival curve. Compression of mortality limits gains in life expectancy when it is already high, even if mortality rates at older ages are declining at a fast pace. This is in keeping with the discussion in section 3.1.3 for why life expectancy at birth becomes a less suitable measure for trends in longevity as it exceeds 80 years of age. The increase in life expectancy at birth from the same rate of improvement in age-specific death rates would be expected to be smaller in a population further down the path to mortality compression than another.

One implication is that a narrowing of the life expectancy differential between two populations is a possible outcome even if their death rates are improving at the same pace; if the age at death distribution for one population is more dispersed than the other. Glei and Horiuchi (2007) demonstrated this for men and women. For the majority of the 29 high-income countries they studied, the recent narrowing of life expectancy at birth between the two sexes was found to be due primarily to sex differences in the age pattern of mortality. The same rate of mortality decline produced smaller gains in life expectancy at birth for women than for men because women's deaths are less dispersed across age, that is, there is less variance in female age at death and female survivorship is more rectangular. The same could be true for two other subgroups of a population with differently-shaped age at death distributions, for example, ethnic groups. Glei and Horiuchi suggest that (p. 155) "...the results of this research seem to illustrate the risk of interpreting observed demographic trends without considering mathematical relationships at the aggregate level." 
From this theory, it is assumed here that past trends in the mortality of population subgroups may inform the extrapolation of population average mortality. Specifically, it is assumed that if mortality trends in subpopulations do not follow the demographic theories of mortality variation, then the extrapolation may be questioned. Table 6.1 summarises the theories relevant to this approach, and the following section applies them to subgroups within the New Zealand population.

Table 6.1: Summary of mortality variance theories as they apply to comparing two subpopulations

\begin{tabular}{|c|c|c|}
\hline & $\begin{array}{c}\text { Subpopulation } 1 \\
\text { (for example females) }\end{array}$ & $\begin{array}{c}\text { Subpopulation } 2 \\
\text { (for example males) }\end{array}$ \\
\hline $\begin{array}{l}\text { Path to mortality } \\
\text { compression }\end{array}$ & More advanced & Less advanced \\
\hline $\begin{array}{l}\text { Age-specific mortality } \\
\text { rates }\end{array}$ & Lower & Higher \\
\hline $\begin{array}{l}\text { Life expectancy at } \\
\text { birth }\end{array}$ & Higher & Lower \\
\hline Survival curve & More rectangular & Less rectangular \\
\hline $\begin{array}{l}\text { Variance in age at } \\
\text { death }\end{array}$ & $\begin{array}{l}\text { Lower at younger ages } \\
\text { Higher at oldest ages }\end{array}$ & $\begin{array}{c}\text { Higher at younger ages } \\
\text { Lower at oldest ages }\end{array}$ \\
\hline $\begin{array}{l}\text { Increase in life } \\
\text { expectancy at birth for } \\
\text { same reduction in } \\
\text { mortality rates }\end{array}$ & Smaller & Larger \\
\hline $\begin{array}{l}\text { Contribution to life } \\
\text { expectancy at birth } \\
\text { increase over time }\end{array}$ & $\begin{array}{l}\text { More from } \\
\text { older ages }\end{array}$ & $\begin{array}{c}\text { More from } \\
\text { younger ages }\end{array}$ \\
\hline
\end{tabular}

Source: Summarised from discussion above with key reference texts: Glei and Horiuchi 2007; Smits and Monden 2009; Tuljapurkar and Edwards 2011; Vaupel 1986. 


\subsection{Reflecting mortality of subgroups within New Zealand}

New Zealand has a unique ethnic mix and a high level of migration. Both of these features continue to add diversity to genetic, cultural, socio-economic and health factors potentially affecting mortality.

\subsubsection{Mortality data by subgroup}

New Zealand is unusual in that official period life tables have been produced for ethnically defined subgroups of the total population - Māori and nonMāori - for decades, and 20-year population projections are produced for four ethnic groups: European or other (including New Zealander), Māori, Asian and Pacific. Of the other countries analysed in this thesis, there may be separate studies of past mortality for subgroups defined by ethnicity or region. Only the US produces life tables by "race" ("White", "Black" and "Hispanic origin") (Arias 2010a, b). None of the countries produces historic cohort life expectancy by ethnicity or race or other grouping. Mortality projections are for total populations in most countries although in the US population projections are produced for "Hispanic", "Non-Hispanic Black" and "NonHispanic all other races" (US Census Bureau 2008).

Age-standardised death rates by ethnic group allow a comparison of summary mortality levels in New Zealand. From Table 6.2 it can be seen that both Māori and Pacific peoples have higher mortality and Asians better mortality than the New Zealand population on average. Table 6.3 shows the size of the ethnic groups within the New Zealand population. 
Table 6.2: Age-standardised death rates by ethnic group, 1996-7 to 2005-7

\begin{tabular}{|l|c|c|c|}
\hline & $\mathbf{1 9 9 6 - 7}$ & $\mathbf{2 0 0 0 - 2}$ & $\mathbf{2 0 0 5 - 7}$ \\
\hline Māori & 11.9 & 11.2 & 9.9 \\
\hline Pacific & 9.0 & 9.1 & 8.8 \\
\hline Total New Zealand population & 7.5 & 6.5 & 5.8 \\
\hline Non-Māori & 7.1 & 6.1 & 5.4 \\
\hline European or other & 7.0 & 6.0 & 5.4 \\
\hline Asian & 5.3 & 4.5 & 3.7 \\
\hline
\end{tabular}

Source: Statistics New Zealand 2009c Table 2.03. Mortality rates for each group at each time period are age-standardised to the New Zealand Estimated Resident Population of June 1996. Other includes "New Zealander".

Table 6.3: Ethnic groups in New Zealand

\begin{tabular}{|lcccc|}
\hline & & & & \\
European & $\mathbf{1 9 9 1}$ & $\mathbf{1 9 9 6}$ & $\mathbf{2 0 0 1}$ & $\mathbf{2 0 0 6}$ \\
\cline { 2 - 5 } Māori & $83 \%$ & $83 \%$ & $80 \%$ & $79 \%$ \\
Pacific & $13 \%$ & $15 \%$ & $15 \%$ & $15 \%$ \\
Asian & $5 \%$ & $6 \%$ & $6 \%$ & $7 \%$ \\
Other & $3 \%$ & $5 \%$ & $7 \%$ & $9 \%$ \\
\hline
\end{tabular}

Source: Census usually resident population count, Statistics New Zealand. People can identify with more than one ethnic group so figures do not add to 100 per cent.

Additionally, there has been significant work in New Zealand on estimated mortality differences between groups using a specially-derived dataset, the New Zealand Census-Mortality Study. Estimated absolute and relative inequalities in mortality associated with ethnicity and socio-economic status have been tracked from 1981-84 to 2001-04 (Blakely et al. 2007; Blakely et al. 2008). The mortality rate for all groups fell, with that for Pacific peoples and Māori falling less than for Asians and others. Absolute differences in agestandardised mortality across ethnic and income groups (ages 1-74) were stable, but relative inequality increased. However, from 1996-99 to 2001-04, relative inequality stabilised and absolute inequality declined, indicating a 
potential reversal of the previous trend of widening inequality. The underlying reasons for these trends are still being investigated. Tobias et al (2009) found an association of the trend in relative inequalities between Māori and non-Māori mortality rates with the trends in social inequalities within the population, with a time lag of a few years. Differential tobacco use, income, ethnicity and gender were found to cumulatively contribute to the gap between Māori and non-Māori period life expectancy at birth (Carter et al. 2010).

Despite the availability of some data by ethnic group within New Zealand, work to investigate mortality variance is hampered by inconsistent ethnic classifications and by a lack of data, especially at older ages. The Statistics New Zealand ethnic life tables provide a robust time series only since 1996. Before that date there was inconsistency in the Census of Population and Dwellings question and death registration forms used to define ethnicity for population numbers and death statistics. Prior to 1996, the wording of the ethnicity question used in the Census changed several times with the "degree of blood" or race being asked for prior to 1986. The "degree of Māori or Pacific Island blood, if any" of the deceased person's parents was requested for deaths registered prior to 1996 (Statistics New Zealand 2009c pp. 2-3). Further, there is evidence of under-reporting of Māori deaths prior to 1995 and consequently over-reporting of non-Māori deaths (Statistics New Zealand 2009c p. 5). From 1996, the ethnic concept used for both population and death statistics is a selfidentified concept of belonging. People can belong to more than one ethnic group, regardless of ancestry or birthplace. Ethnicity is self-identified in the Census, but identified by the person registering a death. It is therefore 
possible that an individual may change his or her own choice of ethnicity during life and then be differently identified at death.

\subsubsection{Māori and Non-Māori mortality}

For the purposes of this analysis, the best source of data was taken to be the official ethnic life tables. This is because an analysis of the variance of mortality according to the theories outlined in section 6.1 needs a full life table for two subpopulations. Therefore, this analysis can only be carried out for Māori and non-Māori groups. The latest data, for 2005-7, is used but data from 1960-62 is also shown for consistency with the period covered in the international comparison of earlier chapters. Interpretation will be made with care given the concerns over robustness of source data.

The 2005-7 New Zealand life tables show that mortality rates are higher (worse) for Māori than non-Māori at all ages for males and females. Figure 6.1 shows this on the same logarithmic scale that Figure 4.3 and Figure 4.4 showed a comparison of the mortality rates of 2006 for the countries selected for this analysis. The gap between the average mortality rates of the two largest ethnic groups of New Zealand is larger than the gap between the average mortality rates of New Zealand and other countries. 
Figure 6.1: Mortality rate at age $x\left(q_{x}\right)$ for ages 0 to 100 years by gender and ethnicity, logarithmic scale, New Zealand population, 2005-7

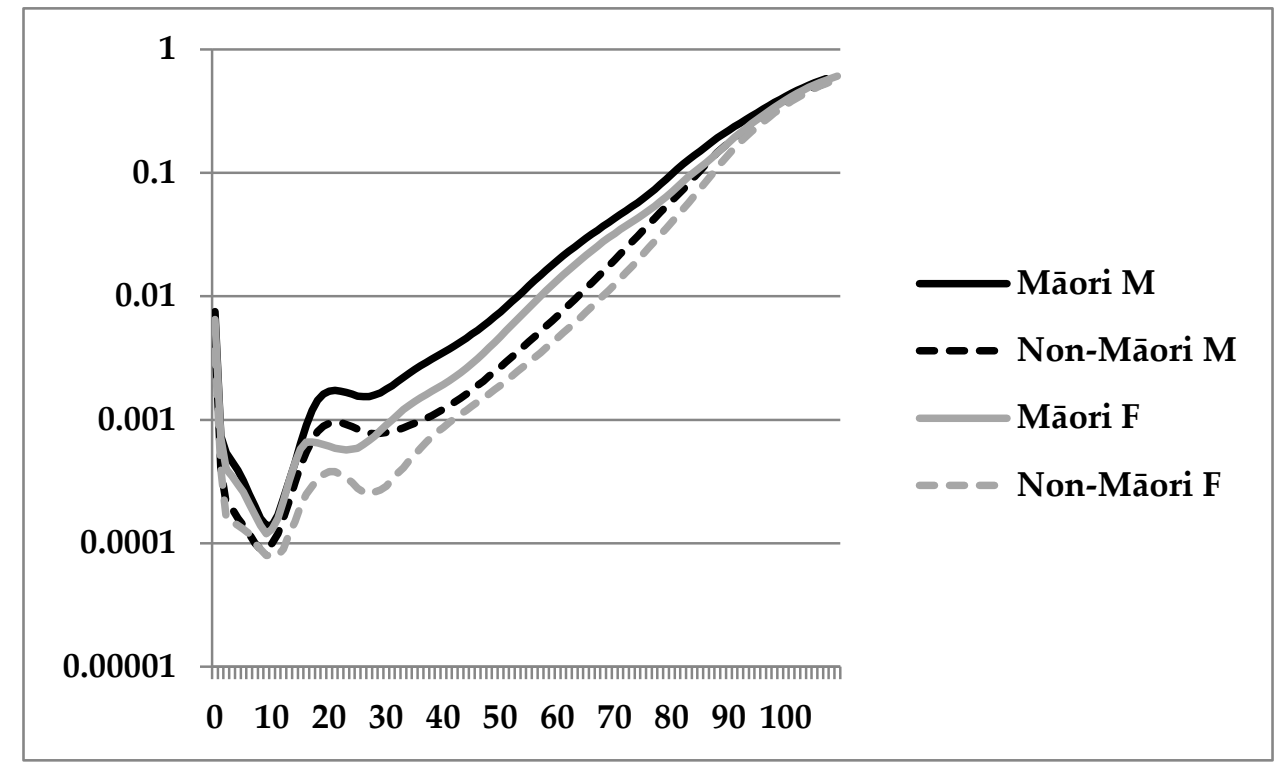

Source: Calculated from 2005-7 life tables Statistics New Zealand (2009c).

Figure 6.2 shows the relative difference in mortality rates by age. From birth to age 40 the age-specific Māori mortality rates are between one and a half times to three times that of non-Māori. However, Table 6.4 shows the numbers of deaths in New Zealand in 2010 by ethnicity and demonstrates that death rate comparisons at ages below 40 are based on few data points, especially for female Māori. Thus the fluctuations in Figure 6.2 are a data artefact of few deaths and very low absolute levels of mortality rates at those ages so that interpretations of excess mortality should be made with caution. After age 40 mortality rates of Māori men are around 3 times that of non-Māori men until around age 60 when they fall to an excess of around ten per cent by age 100. A similar pattern appears for females, except for the overhang of fluctuation shown at the start of this age group. Again at the oldest ages there are few deaths, especially in the Māori group, so proportionality of death rates is imprecise. 
Figure 6.2: Mortality rate at age $x\left(q_{x}\right)$ for Māori as a percentage of that for non-Māori, ages 0 to 100 years by gender, New Zealand population, 2005-7

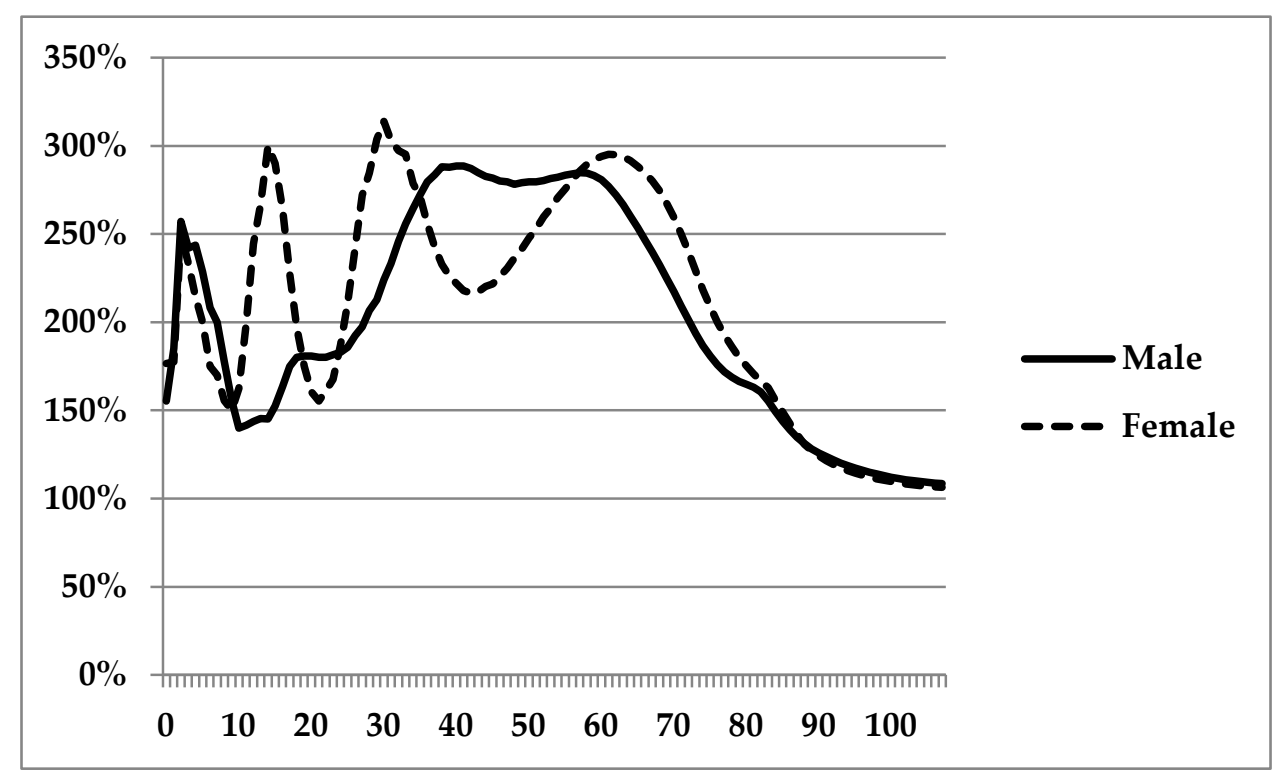

Source: Calculated from 2005-7 life tables Statistics New Zealand (2009c).

Table 6.4: Numbers of deaths registered in New Zealand of New Zealand residents, by age and ethnicity, 2010

\begin{tabular}{|l|cc|cc|c|}
\hline & Males & Females & \multirow{2}{*}{} \\
\cline { 2 - 5 } & Māori & $\begin{array}{c}\text { Non- } \\
\text { Māori }\end{array}$ & Māori & $\begin{array}{c}\text { Non- } \\
\text { Māori }\end{array}$ & Total \\
\cline { 2 - 5 } Age 0 & 70 & 115 & 51 & 89 & $\mathbf{3 2 5}$ \\
Age 1-39 & 207 & 492 & 109 & 284 & $\mathbf{1 , 0 9 2}$ \\
Age 40-79 & 1,051 & 6,333 & 885 & 4,508 & $\mathbf{1 2 , 7 7 7}$ \\
Age 80+ & 147 & 5,808 & 262 & 8,027 & $\mathbf{1 4 , 2 4 4}$ \\
\cline { 2 - 5 } Total & $\mathbf{1 , 4 7 5}$ & $\mathbf{1 2 , 7 4 8}$ & $\mathbf{1 , 3 0 7}$ & $\mathbf{1 2 , 9 0 8}$ & $\mathbf{2 8 , 4 3 8}$ \\
\hline
\end{tabular}

Source: Derived from Statistics New Zealand data 
An alternative presentation to demonstrate the different levels of agespecific mortality rates in the two ethnic subpopulations is the survival curve in Figure 6.3. This shows that the non-Māori subpopulation is further along the transition path to compression of mortality than the Māori population, for both males and females.

Figure 6.3: Survival curve from 100,000 births for New Zealand from period tables 2005-7 for Māori and non-Māori, males and females

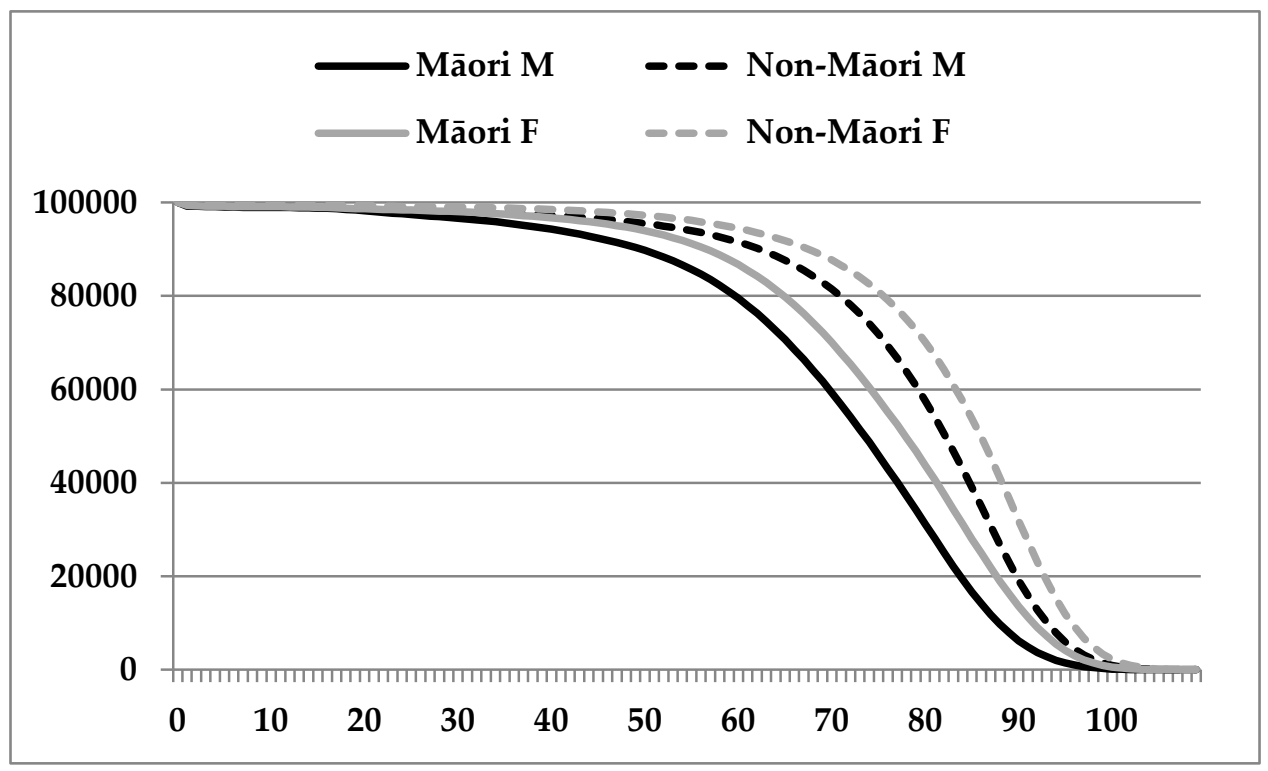

Source: Derived from Statistics New Zealand 2009c

A further comparison of current levels of mortality comes from the summary measure, life expectancy. Period life table data must be used as cohort life expectancies are not produced by ethnicity. Statistics New Zealand (2009c) investigated differentials between Māori and non-Māori period life expectancy since 1950-52 and concluded that despite data inconsistencies over that time period, Māori life expectancies have nearly always been lower than those of non-Māori at all ages, but that the gap in period life expectancy at birth has narrowed over the long term, albeit with short-term fluctuations. 
Table 6.5 gives life expectancies for Māori and non-Māori at selected ages for the two time periods used for the international analysis of this thesis. The caveat for comparisons over time is applies here. However, as expected, improvement in life expectancy over that time can be seen for all subgroups and at all ages. The greater of the two increases in life expectancy at each age over the period - Māori or non-Māori - for each age/gender pairing is highlighted in bold. The increase in life expectancy at birth was better for Māori for both males and females, but for males the improvement was better for non-Māori at all the older ages shown here. For females, greater improvement in life expectancy at ages 75 and 65 for non-Māori compared to Māori is shown. Therefore, although the gap in period life expectancy at birth between Māori and non-Māori did narrow over that time period for both males and females, the gap in male life expectancy at all older ages shown widened. For females, the gap narrowed for ages shown up to 55, but widened for ages 75 and 85 . 
Table 6.5: Period life expectancy at selected ages, New Zealand Māori and non-Māori and the gap between them, 1960-62 and 2005-7 and change over that period

\begin{tabular}{|c|c|c|c|c|c|c|}
\hline & 0 & 10 & 40 & 55 & 75 & 85 \\
\hline \multicolumn{7}{|l|}{ 1960-2 } \\
\hline \multicolumn{7}{|l|}{ Male } \\
\hline Māori & 59.1 & 53.4 & 27.1 & 16.2 & 7.0 & 3.5 \\
\hline Non-Māori & 69.2 & 61.1 & 32.7 & 19.8 & 7.5 & 3.9 \\
\hline Gap & 10.1 & 7.7 & 5.6 & 3.6 & 0.6 & 0.3 \\
\hline \multicolumn{7}{|l|}{ Female } \\
\hline Māori & 61.4 & 54.7 & 27.4 & 16.7 & 7.4 & 4.5 \\
\hline Non-Māori & 74.5 & 66.1 & 37.1 & 23.5 & 8.9 & 4.5 \\
\hline Gap & 13.1 & 11.4 & 9.7 & 6.9 & 1.5 & 0.0 \\
\hline \multicolumn{7}{|l|}{$2005-7$} \\
\hline \multicolumn{7}{|l|}{ Male } \\
\hline Māori & 70.4 & 61.1 & 33.2 & 20.6 & 8.4 & 4.5 \\
\hline Non-Māori & 79.0 & 69.5 & 40.6 & 26.7 & 11.0 & 5.6 \\
\hline Gap & 8.6 & 8.3 & 7.4 & 6.1 & 2.5 & 1.1 \\
\hline \multicolumn{7}{|l|}{ Female } \\
\hline Māori & 75.1 & 65.7 & 36.9 & 23.6 & 10.2 & 5.6 \\
\hline Non-Māori & 83.0 & 73.4 & 43.9 & 29.8 & 13.0 & 6.7 \\
\hline Gap & 7.9 & 7.6 & 7.1 & 6.2 & 2.8 & 1.1 \\
\hline \multicolumn{7}{|c|}{ Change over period } \\
\hline \multicolumn{7}{|c|}{ Male } \\
\hline Māori & 11.3 & 7.7 & 6.1 & 4.4 & 1.5 & 1.0 \\
\hline Non-Māori & 9.8 & 8.3 & 7.9 & 6.9 & 3.5 & 1.8 \\
\hline \multicolumn{7}{|l|}{ Female } \\
\hline Māori & 13.7 & 11.0 & 9.5 & 6.9 & 2.8 & 1.0 \\
\hline Non-Māori & 8.5 & 7.3 & 6.9 & 6.3 & 4.1 & 2.2 \\
\hline
\end{tabular}

Source: Derived from Statistics New Zealand complete period life tables 196062 and 2005-7 (Statistics New Zealand 2009c). Note that data in the two time periods are derived from different definitions of ethnicity, so trends over time should be interpreted with caution. Bold figures show the higher of the Māori and non-Māori data, unrounded. 
To examine further the sources of change in life expectancy at birth, Table 6.6 investigates the age groups from which mortality rate change contributes to life expectancy at birth change. Compared to Māori, the contribution to improvements in life expectancy at birth for the non-Māori population is weighted more towards improvements in mortality at older ages.

Table 6.6: Percentage contribution to instantaneous change life expectancy at birth by age group, New Zealand 2005-7 by ethnic groups and gender

\begin{tabular}{|c|c|c|c|c|c|c|}
\hline & \multicolumn{3}{|l|}{ Males } & \multicolumn{3}{|c|}{ Females } \\
\hline & Total & Māori & $\begin{array}{l}\text { Non- } \\
\text { Māori }\end{array}$ & Total & Māori & $\begin{array}{l}\text { Non- } \\
\text { Māori }\end{array}$ \\
\hline Age 0 & $3.7 \%$ & $3.9 \%$ & $3.3 \%$ & $3.4 \%$ & $3.8 \%$ & $2.9 \%$ \\
\hline Age 1-39 & $12.4 \%$ & $16.6 \%$ & $11.1 \%$ & $7.5 \%$ & $10.4 \%$ & $6.4 \%$ \\
\hline Age $40-80$ & $59.2 \%$ & $68.7 \%$ & $58.5 \%$ & $53.6 \%$ & $67.6 \%$ & $52.3 \%$ \\
\hline Age 81+ & $24.7 \%$ & $10.8 \%$ & $27.1 \%$ & $35.5 \%$ & $18.2 \%$ & $38.4 \%$ \\
\hline Total & $100.0 \%$ & $100.0 \%$ & $100.0 \%$ & $100.0 \%$ & $100.0 \%$ & $100.0 \%$ \\
\hline
\end{tabular}

Source: Calculated from Statistics New Zealand complete period life tables 2005-7 (Statistics New Zealand 2009c). Method for calculating contribution to life expectancy at birth (marginal instantaneous rate change in life expectancy at birth as a ratio to instantaneous mortality rate change, by age) derived from that used in OCA (2009) Appendices E and F.

As demographic theories explain, variance in age at death is related to life expectancy. Table 6.7 shows variance in age at death at or above selected ages for the sub-populations and ages in Table 6.5 at the two time periods of interest. The greater of the two variances - Māori or non-Māori - for each age/gender pairing is highlighted in bold. 
Table 6.7: Variance in age at death at or above selected ages, New Zealand Māori and non-Māori and the gap between them, 1960-62 and 2005-7

\begin{tabular}{|l|cccccc|}
\hline & $\mathbf{0}$ & $\mathbf{1 0}$ & $\mathbf{4 0}$ & $\mathbf{5 5}$ & $\mathbf{7 5}$ & $\mathbf{8 5}$ \\
\hline 1960-2 & & & & & & \\
Male & & & & & & \\
Māori & $\mathbf{2 1 . 0}$ & $\mathbf{1 4 . 7}$ & $\mathbf{1 1 . 0}$ & $\mathbf{8 . 4}$ & 4.1 & 2.0 \\
Non-Māori & 16.0 & 11.7 & 9.9 & 8.3 & $\mathbf{4 . 3}$ & $\mathbf{7 . 7}$ \\
\hline Female & & & & & & \\
Māori & $\mathbf{1 9 . 4}$ & $\mathbf{1 4 . 0}$ & $\mathbf{1 1 . 7}$ & $\mathbf{9 . 2}$ & $\mathbf{4 . 7}$ & 2.8 \\
Non-Māori & 14.4 & 10.2 & 9.4 & 8.1 & 4.7 & $\mathbf{7 . 9}$ \\
\hline 2005-7 & & & & & & \\
Male & & & & & & \\
Māori & $\mathbf{1 4 . 6}$ & $\mathbf{1 3 . 2}$ & $\mathbf{1 0 . 9}$ & $\mathbf{9 . 0}$ & 4.8 & 2.9 \\
Non-Māori & 12.0 & 10.8 & 9.3 & 8.3 & 5.3 & $\mathbf{8 . 4}$ \\
\hline Female & & & & & & \\
Māori & $\mathbf{1 3 . 3}$ & $\mathbf{1 1 . 8}$ & $\mathbf{1 0 . 8}$ & $\mathbf{9 . 4}$ & 5.4 & 3.2 \\
Non-Māori & 10.1 & 8.8 & 8.8 & 8.0 & $\mathbf{5 . 4}$ & $\mathbf{8 . 7}$ \\
\hline
\end{tabular}

Source: Derived from Statistics New Zealand complete period life tables 196062 and 2005-7 (Statistics New Zealand 2009c). Note that data in the two time periods are derived from different definitions of ethnicity, so trends over time should be interpreted with caution. Variance calculated as per Engelman et al (2010). Bold figures show the higher of the Māori and non-Māori data, unrounded.

The non-Māori subgroup, compared to the Māori subgroup, has lower mortality rates, higher life expectancy at birth, a more rectangular survival curve, lower variance in age at death at younger ages but higher at older ages, a smaller increase in life expectancy at birth and a greater contribution to change to life expectancy at birth from older ages. The comparison between the two subgroups is therefore entirely consistent with the tests in Table 6.1. The non-Māori population is further along the pathway to mortality compression than the Māori population and the relative positions are more marked for men than women. 
This analysis provides no evidence to suggest that the mortality trends within the Māori and non-Māori populations, or a comparison between the two, would disturb extrapolation of average total population mortality. Both subpopulations are continuing a path towards mortality compression and that would be taken into account in total population extrapolation.

The paths can be seen as similar to those of males and females forming two sub-populations of the total population. The female subpopulation is more advanced than the male on the path towards mortality compression. Because male and female subpopulations each form roughly half of the total population, there is sufficient data to make robust projections of each subpopulation mortality average. In the case of Māori, Asian and Pacific ethnic groups, official projections are made with a shorter projection period than national projections (20 years instead of over 50 years). The mortality assumptions are not described in sufficient detail to understand the rationale, but the direction of mortality differentials between groups are preserved with apparent narrowing of the gap in period life expectancy at birth, except for an increased advantage to Europeans in the Low Mortality scenario (analysis of Statistics New Zealand 2006-base ethnic projections to 2009-base national projections, 2011-2026). The main scenario of a narrowing differential between all groups and the national average would be consistent with the analysis here. However, with the potential for different ethnicities to be chosen throughout life and at death, and the changes in ethnicity classification over the recent past, the robustness of extrapolation of mortality of each ethnic subpopulation is questioned. 


\subsubsection{Mortality of migrants}

Over one-fifth of New Zealand's population aged 65 and over were born elsewhere (Census 2006 data). Therefore the progress of migrants' mortality in their new country is relevant to future longevity in New Zealand, as is any change in the characteristics of migrants over time. No life tables are produced for migrant groups. The implications of variance in mortality by birthplace on future total New Zealand population mortality are therefore considered more qualitatively than in the case of ethnicity.

A general theory in demography is that migrants are selectively healthy, compared to the average of their country of origin, in order to have made the migration. Selection effects can also arise from health, marital or socioeconomic reasons for migration or return; or from migration making a difference to health risk factors such as moving from a rural to an urban area, or access to health services being made more or less difficult; or from more racial discrimination, or closer family support, in the host compared to birth country. In addition, it is also often assumed that the mortality rates of migrants converge over time to the average of the host country (Lassetter and Callister 2009; Singh and Miller 2004; Wild et al. 2007).

However, the mortality of migrants after long duration of residence in their new country has a small literature, as many studies consider only mortality changes shortly after migration. Convergence to host country mortality has been suggested for migrants from South Asia to England and Wales (Harding 2003), but there was little evidence of this for migrants to Australia after fifteen years residence (Young 1991). Razum and Twardella (2002) suggested that the rate of convergence can be slow if the diseases which 
differ between birth and host country operate at older ages, for example heart disease, as most migration occurs at younger ages. There are therefore many unresolved issues about the progress of mortality rates of migrants, particularly over long durations, and their influence on a population's average mortality.

New Zealand has had a mixture of migrants from countries where population health is both better and worse than New Zealand's. However, most analysis on mortality is by ethnic group rather than birth country. Table 6.2 showed age-standardised death rates by ethnic groups from which it can be hypothesised that mortality is relatively worse for migrants from Pacific Islands than the New Zealand average, but better for migrants from Asia. Jatrana and Blakely (2008) found a similar result for older New Zealand residents. Given that significant inflows from Pacific Island nations began in the 1960s, and that migration from Asian countries increased from the 1980s, a large proportion of people identifying with these ethnic groups are likely to be first generation migrants. Reviewing Table 6.2, 7 per cent of people identified with Pacific ethnicity and 9 per cent with Asian (multiple ethnicities allowed) in 2006. The aggregate influence on the overall population mortality of the higher and lower mortality from these two groups is therefore small.

O'Connell and Dunstan (2009) explored the mortality of migrants from the UK. They concluded that whether or not these migrants had a mortality advantage relative to their country of birth, an advantage relative to New Zealand's average population mortality persists even decades after migration. For all of New Zealand's post-contact history until the 1950s, the United Kingdom was the dominant source country for migration to New Zealand 
(Phillips and Hearn 2008). The UK is still the largest single birth country of overseas-born residents in New Zealand, with six per cent of the resident population being UK-born at Census 2006. The countries chosen for the comparative analysis of this paper were selected because they too received significant British migration. However, if New Zealand were now to have a different profile of migrants compared to these other countries in future, and that affected a significant proportion of the population, then that could result in a divergence of mortality across these countries.

There are arguments against such divergence as a result of migration. The countries selected for analysis here have a diverse profile of current migration and country of birth of current population, as Table 3.4 showed. New Zealand's profile is most like Australia's in terms of size of overseas born population and countries of origin, which provides some reason for $\mathrm{New}$ Zealand not to be an exception. However, if it is the case that migrant mortality moves to the host country norm, then over the long term of the extrapolation period in projections the factors setting the total population average mortality will be more important than migration influences; factors which apply to all developed countries. Alternatively, if migrant mortality disadvantage or advantage persists long term, then the population average will change from that extrapolated only with significant shifts in size and profile of the immigrant population. Such shifts are unlikely to happen in such a short time scale that they are undetected when analysing past data for extrapolative projections. Finally, in the case of all the countries selected here, migration comes from both relatively healthy and unhealthy populations. Migration therefore does not seem likely to be the factor that drives divergence 
in mortality between countries, or be a reason to adjust New Zealand's total population mortality projections.

\subsection{Variation in mortality: New Zealand compared}

Demographic theory suggests that mortality variation can only be compared across countries with the same life expectancy. The countries selected for comparison in this thesis have different life expectancies, so variance cannot be compared simply from one selected country to another. In order to give some insight into New Zealand's comparative position on mortality variance, Figure 6.4 shows New Zealand's performance on a relative measure of variance in lifespan that has been standardised for life expectancy, over the time frame covered by the comparative mortality analysis in this thesis.

The source of data was chosen as the most comprehensive recent database on length of life variation which includes a measure which standardises for life expectancy (at least 50 country $^{*}$ year combinations from at least 10 different

countries). Based on a Gini coefficient of inequality, this relative length of life inequality (RLI) measure:

"... represents the deviation from average length of life inequality at a certain length of life expectancy in units of one standard deviation. Measured in this way, RLI is not correlated with life expectancy and is comparable across different levels of life expectancy".

Smits and Monden (2009) p. 1117 
Figure 6.4: RLI measure for New Zealand length of life variation 1961-2003

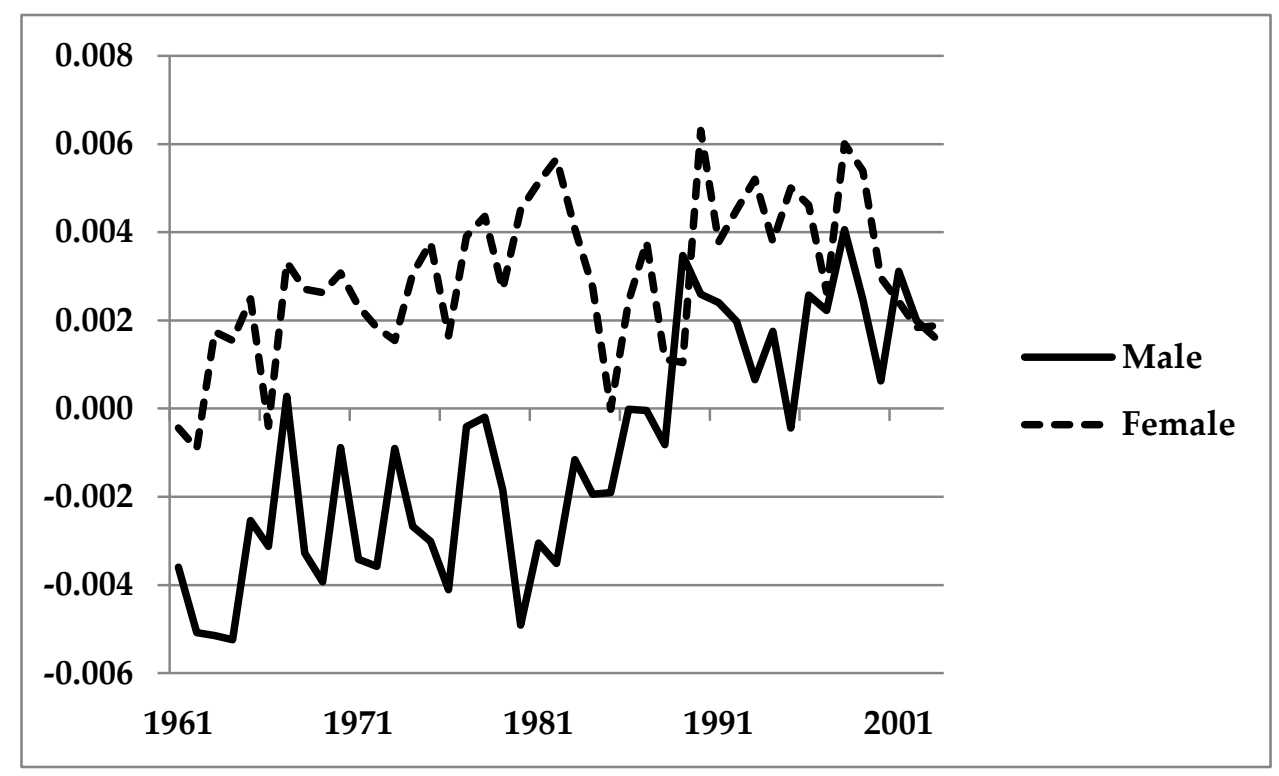

Relative inequality (RLI) measure is the deviation from average length of life inequality at a certain level of life expectancy in units of one standard deviation: the difference between the Gini coefficient of the country* year in question and the mean Gini coefficient of the range.

Source: Downloaded from www.lengthoflife.org (Smits and Monden 2009), August 2010.

Figure 6.4 shows that New Zealand's variation in length of life has worsened relative to other countries since 1961. The measures vary year by year, so short-term trends may not be meaningful, but there is an apparent trend increase in RLI measure over the period. The increase appears particularly sharp for males during the 1980s. That decade saw the inequality for men increase to what had been the prevailing level on the RLI measure for women. This is consistent with the suggestion of Tobias et al (2009) of an increasing contribution of cardiovascular deaths and suicide to ethnic mortality inequality for men in the 1980s.

The current and past position of inequality in New Zealand female age at death inequality may surprise. However, it reflects that comparative data 
from other countries informs assessment of relative heterogeneity in age at death whereas the usual analysis gives only absolute data within one country. The data on the level of New Zealand mortality rates by age and gender in section 4.1 highlighted that the mortality of females aged 1-20 in New Zealand had worsened considerably from 1961 to 2006 relative to all comparator countries, while at the oldest ages New Zealand female mortality compares well. This is consistent with a high degree in variation of age at death for female New Zealanders as compared to other countries, and suggests specific attention be given to why there appear to be too many early female deaths. While out of scope for this thesis, this is clearly a subject worth further research.

\subsection{Conclusions}

Mortality research generally uses average measures of mortality rates such as life expectancy for a population. This thesis is concerned with the plausibility of projections of average lifespan for the New Zealand population. Within demography, the variance of mortality has a smaller literature which focuses largely on theoretical aspects of the variation in lifespan across and within populations. Within epidemiology and public health disciplines the focus is avoidable health inequalities, and in particular the association of such variance with socio-economic position.

Demographic theory shows that life expectancy, the rate of change in agespecific mortality rates and mortality variance are linked. There is some inherent variation in mortality or lifespan in a population. Variation in lifespan will decline as life expectancy increases, except at older ages. For the same reduction in mortality rates, gains in life expectancy will be less for a 
population further advanced in the compression of mortality than another with a less rectangularised survival curve. These characteristics of mortality and life expectancy imply that any correct interpretation of observed trends requires a theoretical grounding in mortality variation to isolate any true deviation from an otherwise expected trend. There is no consensus on the best measure or sets of measures for mortality variation, but more than one measure should be used. Lifespan variance within a population should only be compared with that in populations of the same life expectancy at birth; which means that, for example, the variance in lifespan of the non-Māori population cannot be compared with that of Māori.

For the analytic framework in this thesis, demographic theories of mortality variance theories are used to compare mortality rates, life expectancy and mortality variance between subpopulations of the New Zealand total population. The Māori subpopulation is less advanced than the non-Māori subpopulation on the path to mortality progression, as are males compared to females. Since these pathways, and the supporting empirical data, are to be expected then no evidence is available to suggest that mortality changes are occurring within the New Zealand population likely to disturb the extrapolation of total population mortality trends. However, data definition inconsistencies and fluidity in the ethnic concept for population and death registrations make it difficult to interpret trends and absolute values of mortality by ethnicity in New Zealand. Importantly, the reliance on period life tables and lack of reliable cohort life expectancies by ethnicity means that given life expectancies for Māori and non-Māori understate actual lifespans in this current era of improving mortality. Further, by applying the general result of mortality compression that life expectancy improvement slows as life 
expectancy increases, the gap between realistic lifespans that would be indicated by cohort life expectancies by ethnicity if they existed would be expected to be smaller than the apparent gap in period life expectancies.

More qualitatively, the changing mix of migrants to New Zealand is not likely to disturb the extrapolation of total population mortality trends at least in a short to medium timescale. Further, despite having only a small positive impact on average population life expectancy measures (see Chapter 5), efforts to tackle early deaths where causes can be isolated, such as policy actions to reduce road traffic and other deaths at ages 1-20 where New Zealand has poor relative levels of mortality would also help to reduce mortality variance.

Mortality variation, and the way it changes over time, is clearly a complex subject. Demographic theory can help to inform what may be driving changes in the relationships between measures and suggests caution in expecting all variance can be eliminated. As life expectancy continues to increase and mortality compression continues, there may be apparent increases in mortality variance especially at older ages that are not due to policy or environmental changes, but a mathematical consequence of the changing shape of the survival curve. 
Underestimating lifespans? Why longevity risk exists Chapter 6

This page has been intentionally left blank. 


\section{Chapter 7:}

\section{Surveying subjective lifespans}

Although the existence of longevity risk from individuals underestimating their potential lifespans is known theoretically, there are few full surveys of adult populations providing data on the extent of potential underestimation and its causes.

This chapter first describes the available international evidence, and then discusses findings from these studies. An article based on these first two sections has been published as O'Connell (2011a). Both the methodology and the outcomes of the international literature informed my design of a sample survey of New Zealanders including questions related to expected lifespan. The remainder of the chapter describes this survey. The survey sampling frame, the design of the questionnaire, the wording of the relevant questions on longevity expectations, the variables chosen for analysis and the methods of analysis are described in subsequent sections.

\subsection{International surveys}

The literature on individuals' expectations of their own longevity refers to "subjective longevity expectations", which are gathered by surveys and measured either by subjective probability of survival to a defined age (SPS) or what is usually called "subjective life expectancy" (SLE). SLE measures individuals' expectations of their own lifespan, as they are asked variants of the question "How long do you think you will live?"; the answer being measured in years. Potential confusion from the dual meaning of "expectancy" 
in SLE, and its incorrect application in the term "life expectancy" as discussed in section 3.1, will be avoided by defining here individuals' expectations of their own likely lifespan as "subjective lifespan" or SLS. The term "subjective longevity expectations" is retained to indicate the general sense individuals have for living into later life.

There are at first sight many international reports on aspects of SLS or SPS. Studies based on surveys asking for individuals' expectations for their own longevity are found in the literature of actuarial science, anthropology, demographics, economics, health studies, policy, psychology and statistics. O'Brien et al (2005) reviewed the design and findings of over 20 different studies of longevity expectations, some of which used the same survey data on responses to an SLS or SPS question. Further searching of journal databases revealed 15 more studies.

Many of these studies were based on small samples, selected by region, occupation or other restriction, or were investigating discipline-specific questions on SLS or SPS not relevant to the broad analysis here. For example, a frequently cited early work in economic literature by Hamermesh (1985) used a sample of 411 male white American academic economists and a sample of 363 individuals taken at random from a Midwest telephone directory who were mostly male. An influential work in psychology by Tokor and Murphy (1967) sampled 48 Caucasian college graduates enrolled in a counsellor trainee program. This thesis is concerned with how the adult population of New Zealand estimates subjective longevity, and how that compares with likely outcomes. Therefore, limited or unrepresentative international studies were 
screened out, to leave those based on a nationally representative adult population.

This screening led to the consideration of six main studies:

- The Health and Retirement Study (HRS) is a national longitudinal study in the US. The question on longevity expectations used is a measure of SPS. Hurd and McGarry (1995) was the first work on longevity expectations based on HRS, and others have followed.

- The English Longitudinal Study of Ageing (ELSA) was developed based on the HRS and also uses a question on SPS. The original work on longevity expectations from ELSA is in Banks et al (2004).

- The Ageing, Status and the Sense of Control (ASOC) survey is a national longitudinal study in the US. The question relevant here is a measure of SLS. Mirowsky (1999) is the original study on longevity expectations from this survey.

- One-off studies have been carried out by inserting questions on subjective longevity expectations into surveys organised for other purposes. All the examples here cover Great Britain. Two (O'Brien et al. 2005; Wardle and Steptoe 2003) used a question on SLS. One (Popham and Mitchell 2007) used a question on SPS.

Table 7.1 summarises the critical features of these six studies. 
Table 7.1: Selected studies of SLS and SPS

\begin{tabular}{|c|c|c|c|c|}
\hline Study & Survey & Setting & Sample & SPS or SLS question \\
\hline $\begin{array}{l}\text { Hurd \& } \\
\text { McGarry } \\
(1995,2002)\end{array}$ & $\begin{array}{l}\text { Health and } \\
\text { Retirement Survey } \\
\text { (HRS) first two } \\
\text { waves, } 1992 \text { and } \\
\text { 1994. Longitudinal } \\
\text { survey focused on } \\
\text { health, economic } \\
\text { and social issues. }\end{array}$ & $\begin{array}{l}\text { Face to face } \\
\text { interviews. }\end{array}$ & $\begin{array}{l}\text { Sample size of over 10,000 comprising } \\
\text { men aged 51-65 and women aged 46-61 in } \\
\text { first wave. } \\
\text { Individuals aged 51-61 weighted to be } \\
\text { representative of the national US } \\
\text { population. Additional respondents are } \\
\text { spouses of age-eligible sample. }\end{array}$ & $\begin{array}{l}\text { SPS: "Using any number from } 0 \text { to } 10 \\
\text { where } 0 \text { equals absolutely no chance and } 10 \\
\text { equals absolutely certain, what do you think } \\
\text { are the chances you will live to be } 75 \text { [also } \\
85 \text { ] or more". } \\
\text { A visual scale showed } 0 \text { and } 10 \text { at either } \\
\text { end, } 0 \text { labelled as "absolutely no chance" } \\
\text { and } 10 \text { as "absolutely certain". }\end{array}$ \\
\hline $\begin{array}{l}\text { Banks et al , } \\
\text { (2004) }\end{array}$ & $\begin{array}{l}\text { ELSA first wave, } \\
\text { 2002-3, modelled } \\
\text { on HRS. }\end{array}$ & $\begin{array}{l}\text { Face-to-face } \\
\text { interviews } \\
\text { with self- } \\
\text { completion } \\
\text { questionn- } \\
\text { aires. }\end{array}$ & $\begin{array}{l}\text { Sample size of over } 12,000 . \\
\text { Core sample designed to represent people } \\
\text { aged } 50 \text { and over, living in private } \\
\text { households in England, drawn from } \\
\text { existing health-related study. Additional } \\
\text { respondents are partners of age-eligible } \\
\text { sample. }\end{array}$ & $\begin{array}{l}\text { SPS: "What are the chances that you will } \\
\text { live to be X?" Reference points are set } \\
\text { according to age, for example, } X=75 \text { for } \\
\text { those aged } 50-64 ; X=85 \text { for those aged } 70-74 \text {. } \\
\text { A visual scale showed } 0 \text { and } 100 \text { at either } \\
\text { end, } 0 \text { labelled as "absolutely no chance" } \\
\text { and } 100 \text { as "absolutely certain". }\end{array}$ \\
\hline $\begin{array}{l}\text { Mirowsky } \\
\text { (1999) }\end{array}$ & $\begin{array}{l}\text { First wave of } \\
\text { Ageing, Status and } \\
\text { the Sense of } \\
\text { Control (ASOC) } \\
\text { survey, } 1995 .\end{array}$ & $\begin{array}{l}\text { Telephone } \\
\text { survey. }\end{array}$ & $\begin{array}{l}\text { Over 2,400 respondents aged } 18-95 \text { who } \\
\text { identified themselves as black or white. } \\
\text { Sample was a national probability sample } \\
\text { of US households, but drawn from } \\
\text { English speakers only, and weighted to } \\
\text { compensate for over-sampling seniors. }\end{array}$ & SLS: "To what age do you expect to live?" \\
\hline
\end{tabular}


Table 7.1: Selected studies of SLS and SPS, continued

\begin{tabular}{|l|l|l|l|l|}
\hline Study & Survey & Setting & Sample & SPS or SLS question \\
\hline $\begin{array}{l}\text { O'Brien et al } \\
(2005)\end{array}$ & $\begin{array}{l}\text { Question inserted } \\
\text { into a consumer } \\
\text { omnibus survey in } \\
2004\end{array}$ & $\begin{array}{l}\text { Face-to-face } \\
\text { interviews. }\end{array}$ & $\begin{array}{l}\text { Over 3,900 British adults aged over 16. } \\
\text { Sample weighted to be nationally } \\
\text { representative. }\end{array}$ & $\begin{array}{l}\text { SLS: "I would now like you to think about } \\
\text { other people of the same age and sex of } \\
\text { yourself. To what age would you expect } \\
\text { them to live on average? Estimate if unsure. } \\
\text { And to what age would you expect } \\
\text { yourself to live? Estimate if unsure. }\end{array}$ \\
\hline $\begin{array}{l}\text { Popham \& } \\
\text { Mitchell } \\
(2007)\end{array}$ & $\begin{array}{l}\text { Question inserted } \\
\text { into the 10th wave } \\
\text { in 2001 of the } \\
\text { annual } \\
\text { longitudinal British } \\
\text { Household Panel } \\
\text { Survey (BHPS) of } \\
\text { social and } \\
\text { economic issues. }\end{array}$ & $\begin{array}{l}\text { Face-to-face } \\
\text { interviews. }\end{array}$ & $\begin{array}{l}\text { Over 4,700 British adults aged 25-64. } \\
\text { Sample reflects first wave sampling - } \\
\text { designed to be representative of British } \\
\text { adults aged 16 and over in 1991 - with } \\
\text { attrition and boost since then. }\end{array}$ & $\begin{array}{l}\text { SPS: Respondents were asked whether } \\
\text { they thought it likely that they would live } \\
\text { to be 75 or over (very likely, likely, } \\
\text { unlikely, very unlikely and do not know). }\end{array}$ \\
\hline $\begin{array}{l}\text { Wardle \& } \\
\text { Steptoe } \\
(2003)\end{array}$ & $\begin{array}{l}\text { Question inserted } \\
\text { into monthly } \\
\text { multi-purpose } \\
\text { omnibus survey in } \\
\text { September 2000. }\end{array}$ & $\begin{array}{l}\text { Face-to-face } \\
\text { interviews. }\end{array}$ & $\begin{array}{l}\text { Over 1,600 British adults aged over 16. } \\
\text { Sample was a national probability sample } \\
\text { of British households. }\end{array}$ & $\begin{array}{l}\text { SLS: “Many people feel that they have } \\
\text { some idea about their life span. Thinking } \\
\text { about your life, what age do you think you } \\
\text { will live to?" } \\
\text { Choices given: up to 70, 70 to 79, 80 to 89, } \\
\text { 90 to 99 and over 100." }\end{array}$ \\
\hline
\end{tabular}


Although both the HRS and ELSA surveys are large, and aim to be nationally representative, they are carried out for research into ageing. They sample older adults only, primarily adults originally aged in their fifties and older. The age profile of later waves is likely to be older because these are longitudinal studies (although the sample is boosted). Thus, despite being national in coverage, HRS and ELSA may have limited relevance for understanding questions of subjective longevity for an entire adult population, but were retained here because of their large sample size. Further, there are several reports using HRS data and to exclude it would significantly reduce the findings available for study.

Of the one-off studies in Table 7.1 that aim to be representative of a national adult population, two (Popham and Mitchell; Mirowsky) have in practice limitations to the sample scope, as detailed in the table. The samples of the two remaining studies from Table 7.1 are more likely to be representative of a national adult population, but both look at the same nation, Great Britain. Wardle and Steptoe do not analyse the gap between subjective life expectancies and population measures, but only look at potential correlates with SLS. This leaves O'Brien et al (2005) as the only study identified of a nationally representative adult population that analyses the gap between subjective and actual life expectancy, as measured by a population life table. This report was self-published by the university department of the researchers. It also differs from the other studies as by its question structure it draws attention first to the respondents' views on others' lifespans before asking for expectations of personal lifespan. 
Therefore, while at first sight the quantum of international evidence on SLS or SPS appears helpful, it is not necessarily generalisable. The surveys are often based on limited and possibly selective data. Another adult population outside the US or GB, such as that of New Zealand, may have different longevity expectations because demographics such as ethnicity or age structure, policy messages shaping norms or cultural ways of thinking about longevity may all be different. Further, existing studies have not been made in an appropriate context to explore longevity risk for individual retirement planning or pension policy.

\subsection{Findings from international surveys}

Despite the limitations of the studies described in Table 7.1, their findings suggest some common themes and potential areas to explore for analysis of longevity expectations of the New Zealand population. This section summarises the findings from international surveys on individuals' subjective expectations of longevity. It covers evidence on: first, how longevity expectations compare with population measures; second, whether the longevity expectations of individuals with recognised mortality risk factors reflect a true assessment of the risk; and third, how individuals think about their longevity expectations. 


\section{How do longevity expectations compare with population measures?}

From the authors' own summaries of their findings on subjective longevity expectations in the US, it may seem that people estimate fairly accurately. For example,

"We find that generally ...[subjective probabilities of survival to 75 or 85 ] do aggregate to population probabilities."

Hurd and McGarry (1995) p. S268

"When compared across age groups, average subjective life expectancies track the current-table actuarial estimates well." Mirowsky (1999) p. 977

However, the US studies mainly compared subjective lifespan (SLS) or subjective probability of survival (SPS) to measures in contemporary period tables. These measures were therefore already underestimates as they did not take future mortality improvement into account. British studies found underestimation in SPS and SLS on an aggregate basis, against contemporary period tables and cohort tables which made some assumption for future improving mortality. Further, the population aggregate may not be relevant. All studies found differences in the gap between SPS or SLS and population measure by gender, with men consistently more optimistic. Therefore, the summaries of aggregate data offer only limited insight. Table 7.2 summarises the aggregated findings from the selected studies which compare individual responses on SPS or SLS with the equivalent population average, and comments on gender differences. 
Table 7.2: Aggregate longevity expectations compared to population measures in selected studies

\begin{tabular}{|c|c|c|c|}
\hline Study & $\begin{array}{l}\text { Date of } \\
\text { survey }\end{array}$ & $\begin{array}{l}\text { Population } \\
\text { measure(s) }\end{array}$ & $\begin{array}{l}\text { Findings from aggregated } \\
\text { comparisons }\end{array}$ \\
\hline $\begin{array}{l}\text { Hurd \& } \\
\text { McGarry } \\
(1995,2002)\end{array}$ & $\begin{array}{l}1992 \\
\text { and } \\
1994\end{array}$ & $\begin{array}{l}\text { US period life } \\
\text { table } 1990 \text { used } \\
\text { for both studies, } \\
\text { aggregated for } \\
\text { each gender } \\
\text { allowing for } \\
\text { age. Brief } \\
\text { consideration of } \\
\text { period life tables } \\
\text { 1980-2000 in } \\
\text { first study. }\end{array}$ & $\begin{array}{l}\text { Compared to } 1990 \text { period table men, } \\
\text { mostly aged 51-61, slightly } \\
\text { overestimated SPS to age } 75, \\
\text { although at age } 55 \text { appeared } \\
\text { consistent with the period table } \\
\text { projected for } 2000 \text {. Men } \\
\text { unrealistically overestimated SPS to } \\
\text { age } 85 \text {. } \\
\text { Women in the same age group } \\
\text { underestimated SPS to age } 75, \text { but } \\
\text { SPS to age } 85 \text { was consistent with } \\
1990 \text { period table. }\end{array}$ \\
\hline $\begin{array}{l}\text { Banks et al , } \\
\text { (2004) }\end{array}$ & 2002 & $\begin{array}{l}\text { Period life } \\
\text { tables, } 2003 \\
\text { (region within } \\
\text { GB not stated). }\end{array}$ & $\begin{array}{l}\text { Both men and women, at all ages } 50 \text { - } \\
64, \text { underestimated SPS to age } 75 \\
\text { compared to the period table. } \\
\text { Underestimation greater by women. }\end{array}$ \\
\hline $\begin{array}{l}\text { Mirowsky } \\
\text { (1999) }\end{array}$ & 1995 & $\begin{array}{l}\text { US period life } \\
\text { table 1992, by } \\
\text { age, gender and } \\
\text { "race" (black or } \\
\text { white). }\end{array}$ & $\begin{array}{l}\text { Compared to the period table, white } \\
\text { females' SLS was consistent, black } \\
\text { females overestimated by } 4 \text { years, } \\
\text { white males by } 3 \text { years and black } \\
\text { males by } 6 \text { years. }\end{array}$ \\
\hline $\begin{array}{l}\text { O'Brien et al } \\
(2005)\end{array}$ & 2004 & $\begin{array}{l}\text { GB period and } \\
\text { cohort life tables } \\
2004 \text {, by age, } \\
\text { and gender. }\end{array}$ & $\begin{array}{l}\text { Females' SLS underestimated period } \\
\text { life table by over } 2 \text { years and cohort } \\
\text { table by } 6 \text { years. Men } \\
\text { underestimated by } 1 \text { year and over } 4 \\
\text { years, respectively. }\end{array}$ \\
\hline
\end{tabular}

Note: A period life table is one which assumes each individual experiences the mortality rates of that period throughout remaining life. A cohort table incorporates assumptions for how mortality rates will improve over the course of each individual's life. In the above, US tables are sourced from the Bureau of the Census and GB tables from the Government Actuary's Department. 
The British studies show a consistent theme of underestimation. Banks et al (2004) graphically showed underestimation for both genders and all age groups covered, and reported that the SPS for women aged 60-64 averaged to 65 per cent, which underestimated the then current average survival probability from period life tables of just over 80 per cent. However, the survival probability on cohort measures which assumed some future mortality improvement was over 85 per cent (PPI 2004). O'Brien et al (2005) found a similar picture, with SLS slightly below contemporary period life tables, but significantly below the population cohort measure. The authors concluded that people had a sense of a prevailing wisdom based on current measures of life expectancy but did not appreciate that the future was likely to bring improving mortality. Thus:

"On average, people under-estimate how long they are likely to live [by 4.62 years for males and 5.95 years for females]... they tend to ignore expected mortality improvements".

O'Brien et al (2005) p. 31

The findings for US females in both the Hurd and McGarry (HRS) and Mirowsky (ASOC) studies are consistent with the O'Brien et al hypothesis. Accuracy compared to an earlier period life table than the survey date implies underestimation of actual longevity because of mortality improvement already occurred and likely over respondents' remaining lifespan. However, the overestimates of US males in both studies (and black females in Mirowsky) appear to contradict this. 
Neither US study investigated the reasons for respondents choosing an SPS or SLS. Mirowsky hypothesised that the major demographic groups in the US "share a common image of normal longevity" (p. 978). If so, that image appeared more consistent with the average contemporary longevity of white females than with other groups. The picture is clearly more complicated for individuals than on average for a population. International studies provide mixed evidence that may not be relevant for the New Zealand population. But the evidence does suggest a risk that people underestimate their potential longevity, and that women may suffer more from this longevity risk than men.

\section{Are risk factors reflected in longevity expectations?}

The analytic studies of subjective longevity expectations in demography and health sciences usually investigate whether people have lower expectations for their own longevity if they have higher actual mortality risk. The evidence is limited and mixed, but there appear to be some risk factors more likely to be associated with lower longevity expectations. Table 7.3 shows the risk factors that were found to correlate with the direction of SPS or SLS from the selected surveys described in Table 7.1.

The comments in Table 7.3 summarise from analyses of whether selected other variables in the survey were statistically associated with the response to the SPS or SLS question in the survey in question. The caveats to the use of the survey data need to be considered. These analyses are effectively searching for associations to the answer to one question on longevity expectations; the power of these tests may be low. The studies using the HRS study analysed responses to SPS at age 75 only. Table 7.3 is an illustration of what has been chosen to study and publish, so does not provide a complete picture. Only 
statistically significant effects $(p<.05)$ are shown in both columns. Where different regression models are published, effects that were significantly significant on only some models are not shown in either column.

The first column in Table 7.3 shows whether the group of respondents with a specific risk factor, as measured by the survey in question, on average gave an SPS or SLS that was correctly higher or lower than the overall average. 'Correct' here means that the risk factor in practice does affect population longevity in the same direction. For example, smokers are known to have lower than average longevity so a 'correct' association would show the average smoker giving an SLS or SPS lower than the average for the sample. The second column shows where there was no significant difference in SPS or SLS from the survey average, when there would be a real life difference. No variable was found in these studies where longevity expectations were obviously in the 'wrong' direction. 


\section{Table 7.3: Significant risk factors affecting direction of longevity}

\section{expectations in selected studies}

\begin{tabular}{|c|c|c|c|}
\hline Study & $\begin{array}{l}\text { Survey } \\
\text { base }\end{array}$ & $\begin{array}{l}\text { Risk factors reported in } \\
\text { correct direction }\end{array}$ & Reported no effect \\
\hline $\begin{array}{l}\text { Schoenbaum } \\
(1997) \\
\text { Table } 2 \\
\end{array}$ & $\begin{array}{l}\text { HRS } \\
1992\end{array}$ & Current heavy smoker & $\begin{array}{l}\text { Former or current light } \\
\text { smoker }\end{array}$ \\
\hline $\begin{array}{l}\text { Falba and } \\
\text { Busch (2005) } \\
\text { Table } 3\end{array}$ & $\begin{array}{l}\text { HRS } \\
1992\end{array}$ & $\begin{array}{l}\text { Obesity or overweight for } \\
\text { female non smokers }\end{array}$ & $\begin{array}{l}\text { Obesity or overweight for } \\
\text { males and female smokers }\end{array}$ \\
\hline $\begin{array}{l}\text { Mirowsky and } \\
\text { Ross (2000) } \\
\text { Tables } 3 \& 4\end{array}$ & $\begin{array}{l}\text { ASOC } \\
1995\end{array}$ & $\begin{array}{l}\text { Education level } \\
\text { Past and current economic } \\
\text { hardship } \\
\text { Self-rated health } \\
\text { Current smoker } \\
\text { Level of self-confidence }\end{array}$ & $\begin{array}{l}\text { Income } \\
\text { Medical conditions } \\
\text { Physical impairment }\end{array}$ \\
\hline $\begin{array}{l}\text { Ross and } \\
\text { Mirowsky } \\
\text { (2002) } \\
\text { Table } 1\end{array}$ & $\begin{array}{l}\text { ASOC } \\
1995\end{array}$ & $\begin{array}{l}\text { Surviving same-sex parent } \\
\text { Have informal health } \\
\text { support } \\
\text { Self-rated health } \\
\text { Smoker } \\
\text { Heavy alcohol drinker } \\
\text { Poor nutrition }\end{array}$ & $\begin{array}{l}\text { Surviving opposite-sex } \\
\text { parent } \\
\text { Young children at home } \\
\text { Have emotional support } \\
\text { Obesity } \\
\text { Teetotal } \\
\text { Walk } \\
\text { Strenuous exercise } \\
\text { Private health insurance }\end{array}$ \\
\hline $\begin{array}{l}\text { O'Brien et al } \\
(2005) \\
\text { p. } 26-27\end{array}$ & $\begin{array}{l}\text { One-off } \\
2004\end{array}$ & $\begin{array}{l}\text { Gender } \\
\text { Single } \\
\text { Tertiary education } \\
\text { Self-rated health } \\
\text { Current smoker } \\
\text { Heavy alcohol drinker } \\
\text { Parent's age }\end{array}$ & $\begin{array}{l}\text { Married or widowed } \\
\text { Income } \\
\text { Social class } \\
\text { Ex-smoker } \\
\text { Low or moderate alcohol } \\
\text { drinker }\end{array}$ \\
\hline $\begin{array}{l}\text { Popham \& } \\
\text { Mitchell } \\
\text { (2007) } \\
\text { Table } 1\end{array}$ & $\begin{array}{l}\text { BHPS } \\
2001\end{array}$ & $\begin{array}{l}\text { Gender } \\
\text { Manual occupational class } \\
\text { Education levels } \\
\text { Income } \\
\text { Self-rated health } \\
\text { Moderate to heavy smoker }\end{array}$ & $\begin{array}{l}\text { Non-manual unskilled } \\
\text { occupational class } \\
\text { Father's social class at age } 14 \\
\text { Infrequent smoker }\end{array}$ \\
\hline $\begin{array}{l}\text { Wardle \& } \\
\text { Steptoe (2003) } \\
\text { p. } 442\end{array}$ & $\begin{array}{l}\text { One-off } \\
2000\end{array}$ & $\begin{array}{l}\text { Occupational class } \\
\text { Smoker } \\
\text { Eating fruit less than daily }\end{array}$ & \\
\hline
\end{tabular}


None of the found associations is conclusive, with the exception of gender. All studies found that on average men gave a lower SLS or SPS than women, even if the significance of the direction or accuracy of difference was not tested. At least two studies recorded a significant difference in the right direction between men and women, and none found no effect. It is probably generally well known that women live longer than men; the evidence exists in the increasing ratio of women to men with age in the population of any developed country. It appears that men know this to the extent that on average they have longevity expectations lower than women. This can be consistent with the suggestion from some studies that men tend to have longevity expectations that are higher than current reality for male longevity. It suggests men are more optimistic than women relative to a lower male longevity expectation.

It is also the case that higher socio-economic position and educational level improves longevity, but this is perhaps less widely known. The evidence here suggests a directional link between longevity expectations and educational level, but a mixed picture as regards measures of socio-economic position more broadly. This would be expected insofar as different measures are used. Two British studies found a link to occupational social class (one only to manual occupations), but one found no link. Income was significant in one British study, not in another and not in a US study. The latter found a link to past economic hardship, but one of the British studies found no link to father's occupational social class in childhood. The sum of evidence therefore suggests that longevity expectations tend not to reflect socio-economic factors with the exception of education. 
Self-reported health status was found to be directionally linked with longevity expectations in four studies from both countries, although having a medical condition or physical impairment was not found to be associated in one US study. Studies using HRS have found that respondents with relatively low SPS correctly predict their greater risk of mortality over short periods, from two to 10 years (Hurd and McGarry 2002; Khwaja et al. 2007; Smith et al. 2001). Although the evidence is not conclusive, an association of perceptions of health and mortality seems likely, at least in the short term.

Some evidence suggested that people with health risk factors underestimate the impact of actual risk involved. This analysis requires comparing the SPS or SLS from the survey with life tables that reflect the mortality experience not only of the usual population attributes of age and gender but also the variable in question. The production of such life tables is rare, and their accuracy is necessarily less robust than national population life tables. Schoenbaum (1997), Ross and Mirowsky (2002) and O'Brien et al (2005), despite working from different samples, all suggested that the true excess mortality risk of smokers was generally greater than respondents who smoked, or who used to, expected. Several studies found the direction of longevity expectations was associated with smoking. However, being a heavy current smoker appeared to make more of an impression on longevity expectations than being a light or ex-smoker, even though in both of the latter situations mortality risk would be elevated from population averages.

Further, Falba and Busch (2005) found that being overweight or obese only significantly affected the perceived survival probability for female nonsmokers. Smoking appears a more influential risk factor than obesity. Falba 
and Busch suggested that obese respondents substantially underestimated the excess mortality risk of obesity. However, the sample dates from the 1992 HRS survey of Americans then mostly aged in their fifties. People of different ages and elsewhere may have different knowledge, and knowledge may generally have increased as the health risks of obesity have had significant media attention. An association of longevity expectations with obesity is therefore not proven.

In summary, these studies, despite being based on few datasets, consistently suggest that individuals can vary their subjective longevity expectations in the direction that risk varies. The more widely used risk factors appear to be gender, educational level, self-reported health status and smoking. Other factors may have an effect, but the evidence is limited. None of the studies asked how respondents actually derived their choice of answer to the question on longevity expectations. When a risk factor correlates in the correct direction with SLS or SPS, then authors tend to imply that people are taking the risk factor into account. However, the analyses investigate correlation only, and the mechanism by which people actually think about their longevity expectations is hypothesised.

\section{How do individuals think about their longevity expectations?}

While Mirowsky (1999) suggested that all adults held the same reference group for SLS, O'Brien et al suggested adults saw themselves in a reference group of like people, and adjusted their SLS according to the broadly correct demographic profile of that group. Under this 'normalising' model, male smokers would have an idea of the current average life expectancy of a male smoker, and reflect that it should be lower than a female non-smoker. 
This is at odds with the psychologists' model of SLS, which from very early in the analysis of the subject has been considered "a critical indicator of a highly complex attitude toward an emotionally charged topic" (Tokor and Murphy 1967 p. 21). Under this 'planning for death' model, people adjust their population average estimate of SLS based on personal anxiety about death or experiences of death among family. This theme has been explored in small and selective studies not considered in detail here, such as Hamermesh (1985) and Brouwer and van Exel (2005). These studies illustrate a hypothesis on the basis of significant associations between SLS and age at death of grandparents and parents that there is "huge reliance on forebears' longevity" (Hamermesh 1985 p. 400) when people set their own longevity expectations. An example of anxiety about death being to the forefront when prompted by events is given by Smith (2008) who found an exaggerated lowering of SPS by people in the areas affected by Hurricane Andrew in 1992.

A third model assumes that people thinking about SLS are not thinking about death, but about remaining life, often specifically about planning for retirement. This model, called here 'life planning', underlies further studies based on HRS, which suggest that US adults aged mainly in their fifties and older with low SPS tend to retire earlier and consume in preference to save (Hurd et al. 2003; Salm 2006). Further, van Solinge and Henkens (2009) found later retirement age intentions for those in a select sample of Dutch employees with good pension plans who expected a longer life, based on higher than average scores on a variable combining subjective probabilities of survival to age 75 and beyond age 90 . 
All these models assume that people have some idea of their likely lifespan. Research on subjective longevity expectations has tended to ignore the survey respondents who did not answer the question on SLS or SPS or answered "don't know". Only 2 per cent non-response was reported in the first wave of HRS (Hurd and McGarry 1995). However, Mirowsky (1999) reported a non-response rate of 21 per cent and O'Brien et al (2005) that 10 per cent of the sample responded "don't know" to the SLS question. The "don't knows" can therefore form a sizeable group. Of the surveys covered in this review, only Mirowsky (1999) made explicit allowance for those responding "don't know", imputing a response to them. No other analysis has been found which profiles the "don't knows" compared to those who responded, or those who appeared to misunderstand the question. Such an analysis could shed light on the characteristics of those more or less likely to have thought about their own longevity, and the information that could help people form more accurate longevity expectations.

\subsection{Structuring the research questions}

The international studies provide useful learning for survey and analysis design, and give confidence that asking for SLS or SPS yields usable responses. The remainder of this chapter describes how the design of these international surveys informed the design of the survey undertaken in New Zealand, thus allowing the comparison of the first New Zealand data on longevity expectations to be compared with the international dataset. Table 7.4 summarises the way the survey and analysis were structured, and references the sections in following chapters which contain the analysis of each research question. 
Table 7.4: Research objectives for survey of New Zealanders' longevity expectations

\begin{tabular}{|c|c|c|c|}
\hline Research gaps & $\begin{array}{l}\text { Research questions } \\
\text { focusing survey }\end{array}$ & $\begin{array}{l}\text { Survey } \\
\text { question } \\
\text { number }\end{array}$ & $\begin{array}{l}\text { Section } \\
\text { contain- } \\
\text { ing } \\
\text { analysis }\end{array}$ \\
\hline $\begin{array}{l}\text { New Zealanders' } \\
\text { subjective longevity } \\
\text { expectations }\end{array}$ & $\begin{array}{l}\text { What do New Zealanders } \\
\text { say when asked for their } \\
\text { subjective longevity } \\
\text { expectations? } \\
\text { How do these expectations } \\
\text { compare with population } \\
\text { benchmarks? }\end{array}$ & Q61 & 8.1 \\
\hline $\begin{array}{l}\text { How longevity } \\
\text { expectations are } \\
\text { formed }\end{array}$ & $\begin{array}{l}\text { How much have New } \\
\text { Zealanders thought about } \\
\text { their own longevity? } \\
\text { How do New Zealanders } \\
\text { choose a response when } \\
\text { asked what they think } \\
\text { their longevity will be? }\end{array}$ & $\begin{array}{l}\text { Q60 } \\
\text { Q62 }\end{array}$ & 8.2 \\
\hline $\begin{array}{l}\text { The profile of } \\
\text { longevity } \\
\text { underestimation in } \\
\text { New Zealand }\end{array}$ & $\begin{array}{l}\text { What predicts high or low } \\
\text { longevity expectations? } \\
\text { What is the profile of those } \\
\text { most likely to under- or } \\
\text { over-estimate longevity? }\end{array}$ & $\begin{array}{l}\text { Analysis } \\
\text { of } \\
\text { responses } \\
\text { to Q61 }\end{array}$ & 8.3 \\
\hline $\begin{array}{l}\text { How longevity } \\
\text { expectations relate } \\
\text { to retirement plans }\end{array}$ & $\begin{array}{l}\text { Do New Zealanders } \\
\text { consider longevity when } \\
\text { making retirement plans? } \\
\text { What is the distribution of } \\
\text { intended retirement age } \\
\text { and implied length of } \\
\text { retirement? }\end{array}$ & $\begin{array}{l}\text { Q34 } \\
\text { Q35 } \\
\text { Q63 }\end{array}$ & 9.1 \\
\hline
\end{tabular}


The first research gap to be addressed was how New Zealanders view their expected longevity and whether, collectively, the population underestimates. The subjective longevity expectations, gathered by survey, are compared to benchmark population measures to see whether the aggregate shape of longevity expectations in New Zealand is similar to the international picture.

Second, the mechanisms by which people use understanding of their own risk factors or other considerations when setting longevity expectations are unknown. This research explores how New Zealanders' longevity expectations are formed. Important questions are how people think about their likely longevity; whether they think about it at all, and in what context. Do people of different characteristics think about their longevity differently, and if so, which factors are different groups most likely to take into account? Are there any groups who are more likely to answer "don't know" when asked for their longevity expectation? Do any of the three models postulated for how people think about longevity - normalising, planning for death or life planning - fit the New Zealand population?

Third, this research aims to describe the extent of longevity underestimation in New Zealand. The characteristics of New Zealanders most likely to expect high or low longevity will be identified, as will the profile of those most likely to under- or overestimate longevity.

Fourth, the research gap on how people think about their likely longevity seems important when considering how to formulate effective communication of information on longevity. A government might be interested in this either 
to help individuals with retirement planning or to communicate the rationale for superannuation policy changes. The few studies to link longevity expectations with retirement planning suggest that longevity underestimation is important, because it may lead people to retire without providing for the potential length of retirement. Hence this research asks how New Zealanders' longevity expectations relate to their retirement plans.

\subsection{Survey sampling frame}

The ANZ-Retirement Commission Financial Knowledge Survey was first carried out in 2006 and repeated in 2009. The Retirement Commission, now known as the Commission for Financial Literacy and Retirement Income, and abbreviated here as CFLRI, is responsible for financial education in New Zealand. It organises the survey with ANZ Bank as the major sponsor. The objective of the survey to measure levels of financial knowledge in the New Zealand adult population so that knowledge levels can be tracked over time and areas of low financial literacy can be identified and addressed.

The survey was carried out by Colmar Brunton, a market research company, under the oversight of a steering group including representatives of the CFLRI, ANZ Bank, and academic and financial experts. I attended most steering group meetings for the 2009 survey as an advisor on financial literacy. In addition, I designed the additional survey questions on longevity expectations to be explained in the next subsection. Colmar Brunton was responsible for sampling and interviewing, using a questionnaire developed by the steering group and described in the next subsection. Details are available in Colmar Brunton (2009), the company's report to its client, then 
called the Retirement Commission, which is the source for all information about the survey in this section unless otherwise stated.

A stratified sample of Statistics New Zealand area units was used, with unit areas selected with probability in proportion to size. Eight face to face interviews were carried out in randomly chosen households in each area unit. A single respondent was chosen at random within each household from those eligible - adult aged 18 and over - by asking for the adult with the last birthday. The ethical procedures for sample selection and interviews followed were those of Colmar Brunton's established practice. The interviews took place during March and April 2009 and were on average 60 minutes long. Each participant was given $\$ 20$.

A sample size of 850 interviews was achieved. The response rate was 62 per cent, considered high by Colmar Brunton relative to other general public surveys which are usually carried out by telephone (personal communication, June 2010). National surveys carried out in New Zealand for statistical purposes, such as the Household Economic Survey (Income), the Survey of Family, Income and Employment and the Household Labour Force Survey have higher response rates in the order of 75-88 per cent. However, these are very large samples ( $\mathrm{n}=4,700,15,100$ and 15,000 households respectively) and supported by Crown budgets. Data to enable analysis of the source of nonresponse in the Financial Knowledge Survey were not collected. However, the potential for non-response bias was mitigated to some degree as the data were proportionally weighted, but not rescaled, to achieve population characteristics as at Census 2006 by household size, age and gender. The analysis in this thesis uses the weights determined by Colmar Brunton. 


\subsection{Survey questionnaire}

The full questionnaire for the 2009 survey consisted of 98 questions, some of which had many parts. The majority of the questions for the 2009 survey were the same or slightly changed from those in the 2006 survey, because of the requirement to track knowledge level results over time.

The first 59 questions in the questionnaire were mainly set by the previous survey; a mixture of specific tests for financial knowledge such as:

Q32) If Nicky has $\$ 100$ in a savings account and the interest rate was $2 \%$ per year, after 5 years how much would Nicky have in her account if she left the money to grow? Would it be more than $\$ 102$, exactly $\$ 102$ or less than $\$ 102$ ?

Q 36) At what age are people entitled to NZ Super?

...and questions on the financial behaviour of the respondent, for example:

Q13) Do you have a budget?

Q34) To what extent have you thought about your financial planning for your retirement?

These questions covered the following subjects, in this order: money management, budgeting, goal setting and financial planning, debt management, mortgages, managing risk, saving, retirement planning, investing, consumer rights and responsibilities, attitudes and behaviours to saving and spending, and locus of control in life. There then followed the 
questions inserted to the 2009 survey to ask about longevity expectations: Q60 to Q63 inclusive.

Following these questions were four questions on KiwiSaver, also not asked in 2006, and then the final 28 questions which collected demographic information about the respondent and information on aspects of their personal finances.

\subsection{Survey questions on longevity expectations}

The questions on longevity expectations were inserted into the 2009 survey specifically for this thesis. I designed these questions, using the context as described in Table 7.4, and learning from the international studies on longevity expectations already covered. This subsection explains the design of these questions, which then are set out in Table 7.5.

All the international studies described in Table 7.1 situated questions on longevity expectations into long questionnaires which were primarily designed around a purpose other than longevity; either health, ageing or consumer interests. It is possible the preceding health related questions in HRS and other international studies tended to concentrate the respondents' thoughts on their perceived good or poor health, which also affected their longevity expectations. It is also possible that the questions preceding those on longevity expectations in the New Zealand survey influenced the respondents differently, towards appropriate financial planning attitudes. However, the format of the New Zealand survey, with longevity expectations questions situated within a questionnaire asking about financial knowledge and behaviour is comparable in form with international studies. 
The international studies gave confidence that asking for longevity expectations - either subjective lifespan (SLS) or subjective probability of survival (SPS) - yields usable responses. However, there were three main decisions to take in question design, for which the international surveys took varied approaches: whether to ask for SLS or SPS; whether to allow responses in single years of age or in age bands; and, how exactly to phrase the questions.

\section{SLS or SPS?}

Both SLS and SPS data can be used to compare expectations with reality and to examine how that differential varies with demographic and other characteristics. In an influential paper on expectations measures in attitudinal surveys for the purpose of econometric modelling, Manski (2004) called for expectations to be measured using subjective probabilities, asserting that is consistent with modern economic theory. He favoured SPS over SLS as SPS reflects that people hold "a set of subjective distributions for an unknown event" (page 1369) rather than expect one deterministic outcome.

However, SLS questions elicit more data that can be analysed in more depth than SPS questions. With SPS, responses yield data only in relation to the ages chosen as benchmarks: usually 75 or 85 . A survival distribution by age has to be modelled using these one or two data points, which demands a lot from analysis of little data (for example as in Khwaja et al. 2007; Perozek 2008). Both SLS and SPS studies suffer from bunching at focal values. O'Brien et al (2005) reported bunching of SLS at quinquennial ages, especially age 80. In the HRS first wave, bunching at probabilities of $0,0.5$ and 1 was reported 
(Hurd and McGarry 1995). Additional inconsistency is reported in SPS studies. In the first wave of HRS, only 70 per cent of respondents chose a lower probability of survival to age 85 than to age 75 . There is no explanation for the "cognition error or misunderstanding ...revealed by the other 30 percent of respondents" (Hurd and McGarry 1995 p. S277). Technically, SLS appears the preferable variable.

Further, the SLS of respondents form a distribution of expected lifespans; the basic unit examined for this study which takes an individual life course perspective. For example, SLS can be compared directly to expected age at retirement. Therefore, SLS was chosen as more relevant for this study.

\section{Age band or single year of age?}

Further consideration was given to how to collect responses from the SLS question (Q61): to ask an open question and collect single age responses or band responses into age brackets; or to prompt a response by showing a card with quinquennial ages or age bands. Using a showcard was preferred in order to be consistent with the way in which showcards were used to collect responses from other questions in the survey. Initially, 5-year age bands (for example $60-64,65-69, \ldots$ ) was chosen in an attempt to avoid bunching at round numbers. However, pre-survey qualitative cognitive testing of the questionnaire showed that the use of single years of age in the showcard was preferred by respondents, who perhaps found age bands confusing when asked for SLS as a year of age. It was decided to use put quinquennial single years of age on the showcard (under $60,65,70$ and so on.). While this introduced bunching, the evidence of the international surveys suggested that bunching was bound to occur anyway. 
Underestimating lifespans? Why longevity risk exists Chapter 7

The same method was used for the question on expected retirement age (Q63), with a similar but younger range of ages offered. This was to give the respondent consistency of question style and to allow the calculation of the gap between SLS and retirement age for each respondent.

\section{Phrasing of longevity questions}

The questions on subjective longevity, as originally developed to meet the research objectives, were tested in a pre-survey qualitative cognitive pilot, and amended slightly to flow with the remainder of the questionnaire. The final wording of the entire section of the questionnaire on longevity expectations is set out in Table 7.5. 
Table 7.5: Questions on longevity expectations in New Zealand survey

The next section is a short one about retirement and ageing.

IF RESPONDENT HAS REPORTED A MAJOR HEALTH PROBLEM

AT Q55 (pg 30), THEN READ: When I read the questions, if you don't feel comfortable answering them, please just let me know and we will skip them.

Q60) Thinking now about what age you might live to, which of the following best describes how much you have thought about it?

SHOWCARD.

CODE ONE ONLY.

I've thought about it a lot

I've given it some thought

I've not really thought about it

I've never thought about it

Don't know

Refused

Q61) What age do you think you will live to?

SHOWCARD.

CODE ONE ONLY.

60 or under

65

70

75

80

85

90

95

100

Over 100

Don't know

Refused 
Table 7.5: Questions on longevity expectations in New Zealand survey, continued

Q62) For what reasons do you say that? OFFER NO PROMPTS.

CODE FIRST MENTIONED IN FIRST COLUMN;

CODE ALL OTHER MENTIONS IN SECOND COLUMN

That's about average/normal [for someone like me]

Genetics

Thinking about my parents

Thinking about my grandparents

Thinking about people my age/that's about normal for people my age

I smoke

I don't smoke

I'm fit/I exercise

I'm not fit/I don't exercise

I have a good diet

I have a bad diet

I'm healthy (generally)

I'm not healthy (generally)/I'm ill

I have had health scare(s)

Medical Science/new technologies

None / No other mentions

Other (SPECIFY)

Don't know

Refused

Q63) At what age, if any, do you think you are most likely to retire? By 'retire' I mean to stop full-time paid work with no intention of working full-time again, although you might still do a little part-time work or some voluntary work.

SHOWCARD.

CODE ONE ONLY.

60 or under

65

70

75

Over 75

Never: I'll carry on working

I've already retired or I don't work

Don't know

Refused 
The CFLRI requested that the questions on longevity not be asked if a respondent had reported earlier in the survey having had a major health problem in the last 12 months. This was in case a very ill respondent would be upset by being asked the age to which he or she might live. In the event, these questions were asked to all survey participants.

Question Q60 on how much the respondent had thought about the age he or she might live to was asked with a showcard with a 4-point Likert scale, and clearly had to be asked before the question asking for an SLS. Question Q62 aimed to elicit why the respondent had chosen the SLS in Q61. An open question with no showcard was chosen because no evidence was found from prior studies even for how many reasons people might have. The first reason reported was isolated and other reasons given collected in another variable.

In addition to the questions in Table 7.5, questions used in the 2006 Financial Knowledge survey were repeated and are included in the analysis here as they enable investigation into the links between SLS and retirement planning.

Q34) To what extent have you thought about your financial planning for your retirement?

(SHOWCARD: A lot, a fair amount, A little, Not at all)

Q35) What are all the things that a person needs to consider when they think about saving for retirement? Anything else? (Unprompted) 


\subsection{Choice of independent variables for analysis}

The Financial Knowledge survey gathered data on a large number of variables. A subset of variables was selected in order to focus the analysis to what is most relevant for the research questions in this thesis. This subset formed the independent variables tested for association with the responses to the longevity expectations questions.

Previous studies explored the association of longevity expectations with demographic characteristics and health risk factors (Table 7.3), both of which are relevant for this research. Because of the focus of the Financial Knowledge survey, a third category of variables on planning and financial knowledge was also available. Some of the variables used were transformed by grouping answers in order to have significant numbers in cells for analysis. Generally, information on don't knows were kept, and merged with refused. There were very few refused. The grouping followed that used in previous reports on the survey, where possible. The data gathered in the survey but excluded from this research were those designed for tracking financial knowledge levels and debt behaviour which were already the subject of separate reports (Colmar Brunton 2009). 


\section{Demographic variables}

For this study, age, gender and education level and ethnicity were available. Two additional available variables were included to explore other angles of ethnicity: whether born in New Zealand and comfort with English. Indicators used for socio-economic status were education level, personal income and net wealth. The latter variable was constructed by Colmar Brunton from response to questions on assets, housing equity and debts.

Table 7.7 sets out the characteristics of the weighted sample on the demographic variables selected and grouped, and compares with the New Zealand adult population as close in time as possible to that of the survey. The weighted sample provides a close representation of the adult population of New Zealand by gender, age, ethnicity and personal income. Differences are within sample error (see Table 7.6) except for a slight over-representation of adults educated at tertiary level.

Table 7.6: Estimated sample errors, full sample, $95 \%$ confidence level, assuming simple random sample

\begin{tabular}{|l|c|}
\hline Survey result & Margin of error \\
\hline & \\
$10 \%$ or $90 \%$ & $+/-2.0 \%$ \\
$20 \%$ or $80 \%$ & $+/-2.7 \%$ \\
$30 \%$ or $70 \%$ & $+/-3.1 \%$ \\
$40 \%$ or $60 \%$ & $+/-3.3 \%$ \\
$50 \%$ & $+/-3.4 \%$ \\
\hline
\end{tabular}


Table 7.7: Demographic characteristics of sample

\begin{tabular}{|c|c|c|c|}
\hline \multirow[b]{2}{*}{ Demographic category } & \multirow{2}{*}{$\begin{array}{l}\begin{array}{l}\text { Sample } \\
\mathrm{n}=850 \\
\text { Weighted } \\
\text { data }\end{array} \\
\text { Per cent }\end{array}$} & \multicolumn{2}{|c|}{$\begin{array}{l}\text { NZ population aged } \\
18 \text { and over }\end{array}$} \\
\hline & & Per cent & Source \\
\hline $\begin{array}{l}\text { Gender } \\
\text { Male } \\
\text { Female }\end{array}$ & $\begin{array}{l}48.0 \\
52.0\end{array}$ & $\begin{array}{l}48.3 \\
51.7\end{array}$ & $\begin{array}{l}\text { Total } \\
\text { estimated }\end{array}$ \\
\hline $\begin{array}{l}\text { Age } \\
18-29 \\
30-44 \\
45-59 \\
60+\end{array}$ & $\begin{array}{l}21.1 \\
30.0 \\
26.2 \\
22.7\end{array}$ & $\begin{array}{l}22.2 \\
27.5 \\
26.4 \\
23.9\end{array}$ & $\begin{array}{l}\text { resident } \\
\text { popul- } \\
\text { ation } \\
\text { June } 2009\end{array}$ \\
\hline $\begin{array}{l}\text { Ethnicity (multiple response) } \\
\text { European or other } \\
\text { Māori } \\
\text { Pacific } \\
\text { Asian } \\
\text { Mid East/Latin American/African }\end{array}$ & $\begin{array}{c}78.2 \\
13.1 \\
5.3 \\
9.5 \\
0.5 \\
\end{array}$ & $\begin{array}{c}78.3 \\
11.8 \\
5.5 \\
9.7 \\
0.8 \\
\end{array}$ & $\begin{array}{l}\text { Census } \\
2006 \\
\text { See text }\end{array}$ \\
\hline $\begin{array}{l}\text { Place of birth } \\
\text { Born in New Zealand } \\
\text { Born outside New Zealand }\end{array}$ & $\begin{array}{l}72.3 \\
27.7\end{array}$ & $\begin{array}{l}70.0 \\
30.0\end{array}$ & $\begin{array}{l}\text { Census } \\
2006\end{array}$ \\
\hline $\begin{array}{l}\text { Comfort with English } \\
\text { Very } \\
\text { Somewhat } \\
\text { Not very or not at all }\end{array}$ & $\begin{array}{c}92.3 \\
5.8 \\
1.9 \\
\end{array}$ & & $\mathrm{n} / \mathrm{a}$ \\
\hline $\begin{array}{l}\text { Education } \\
\text { Primary or basic secondary } \\
\text { Secondary school } \\
\text { Tertiary or postgraduate }\end{array}$ & $\begin{array}{l}21.5 \\
23.6 \\
53.9 \\
\end{array}$ & $\begin{array}{l}25.0 \\
23.6 \\
49.7 \\
\end{array}$ & $\begin{array}{l}\text { NZ } \\
\text { Income } \\
\text { Survey } \\
\text { June 2009 }\end{array}$ \\
\hline $\begin{array}{l}\text { Personal income } \\
\text { Up to and including } \$ 30,000 \text { pa } \\
\text { Over } \$ 30,000 \text { pa } \\
\text { Refused } \\
\text { Don't know }\end{array}$ & $\begin{array}{r}47.2 \\
42.7 \\
7.2 \\
2.9\end{array}$ & $\begin{array}{l}\text { Median } \\
\$ 28,000\end{array}$ & Ages 15+ \\
\hline $\begin{array}{l}\text { Net wealth } \\
\text { Negative } \\
0-\$ 100 \mathrm{k} \\
\$ 101 \mathrm{k}-\$ 300 \mathrm{k} \\
\$ 301 \mathrm{k}-\$ 600 \mathrm{k} \\
\$ 601 \mathrm{k}+\end{array}$ & $\begin{array}{l}24.7 \\
20.3 \\
18.3 \\
21.3 \\
15.4\end{array}$ & & $\mathrm{n} / \mathrm{a}$ \\
\hline
\end{tabular}


Ethnicity is measured in Table 7.7 as in the usual population measures. Both the survey and the New Zealand Census 2006 allowed multiple responses, as people can belong to one or more ethnicity. Responses are counted for each ethnicity, so that the data points for ethnicity add up to more than the number of people in the population and the percentages for ethnicity in Table 7.7 add up to more than 100 per cent. For analysis, the ethnicity variable was grouped differently, so that each respondent is counted once. Respondents who gave a single ethnicity in one of the largest four groups New Zealand European, Māori, Pacific or Asian - form separate groups, as do those who gave both New Zealand European and Māori ethnicities. Those with other multiple ethnicities are included in "All other", along with those who give a single ethnicity that is not in one of the largest groups. The frequency table on this basis is shown as Table 7.8. The sample size for all ethnic groups other than New Zealand European is still smaller than ideal for a full analysis of results by ethnicity.

Table 7.8: Main single and dual ethnic groups

\begin{tabular}{|c|c|c|}
\hline \multirow[t]{2}{*}{ Ethnicity } & \multicolumn{2}{|c|}{$\begin{array}{l}\text { Sample, } n=850 \\
\text { Weighted data }\end{array}$} \\
\hline & nequiv & Per cent \\
\hline Sole New Zealand European & 552 & 65.0 \\
\hline Sole Māori & 67 & 7.9 \\
\hline NZ European and Māori & 38 & 4.5 \\
\hline Sole Pacific & 35 & 4.1 \\
\hline Sole Asian & 77 & 9.1 \\
\hline All other & 80 & 9.4 \\
\hline
\end{tabular}

Source: Analysis of Financial Knowledge 2009 survey responses 


\section{Health risk variables}

Data was not collected directly on health status or risk factors except where the respondent gave in response to Q62, the reason for choosing an SLS a risk factor such as "I smoke" or "I have a good diet". As the survey was about financial knowledge rather than health, it would not have been appropriate to gather the health-related data available to international surveys such as the HRS or ELSA. Therefore, the analysis of self-reported health risk factors in this study is limited to where they are top of mind for respondents. This was designed to be the case in order to identify specifically which, if any, health risk factors were in mind when respondents gave their longevity expectations. This effect has only been inferred in previous studies. The use of this variable is described further in section 8.2.2.

\section{Planning and knowledge variables}

Variables that measured respondents' engagement with retirement planning and planning for later life were selected. These were whether the respondent was currently contributing to KiwiSaver; whether he or she had made a will and how much he or she had thought about financial planning for retirement. In addition, in order to check associations with the general attitude towards planning rather than leaving things to 'fate', three variables on locus of control were selected. These asked the respondent to agree or disagree on a 5-point Likert scale to "My life is determined by my own actions"; "...is determined by things beyond my control"; and, "...is controlled by the actions of other people". Responses were grouped to three categories representing agree; neither agree or disagree; and disagree. 
Finally, variables that conveniently measured overall financial knowledge were selected. The major summary measure of the Financial Knowledge survey was membership of the High, Medium or Low knowledge group. This measure had been calculated by scoring for correct answers on the basic financial knowledge questions in the survey, across a variety of financial topics, then using those scores as the basis of splitting into the three groups. The scores from the 2006 Financial Knowledge survey were used then to divide the sample into three evenly sized groups, named 'tritiles'. In 2009 the same boundary scores for each group were maintained, in order to track the desired increasing number in the High knowledge group and decreasing number in the Low knowledge group.

Being in the High knowledge group is more likely for those aged 35-64 rather than older or younger, New Zealand European, tertiary educated, home owners, skilled executives or professionals, with an annual household income of more than $\$ 50,000$. Being in the Low knowledge group is more likely for those aged 18-24 or 65 and over, of Māori or Pacific ethnicity, primary or basic secondary educated only, tenants, not in paid employment or in semi-skilledor unskilled work, and on a low income (ANZ-Retirement Commission 2009 p. 9). Further, financial product ownership is highest among the High knowledge group and lowest among the Low knowledge group (Colmar Brunton 2009 p. 15). Further tests (not shown) confirm that membership of financial knowledge group is strongly associated with contributing to KiwiSaver, having made a will and having thought about planning for retirement (all $\mathrm{p}<.001)$. Thus, membership of a knowledge group is associated with both socio-economic status and with activity in personal finance management. 
Further, two knowledge groups had been defined by the steering group at 'extreme' scores. The Lowest knowledge group were the 10 per cent of respondents with the lowest scores on the basic financial questions, and can be seen as a marker for those excluded from personal finance management. Conversely, the Advanced knowledge group scored highly on knowledge questions about advanced financial topics, mostly investing. Around 20 per cent of respondents were in the Advanced knowledge group. Table 7.9 shows the distribution of the sample on these planning and financial knowledge variables. Note that the proportion in the High knowledge group had increased from 2006 to 2009 as desired, with less than a third of the sample in the Low and Medium knowledge groups.

The sample proportions reporting contributing to KiwiSaver were slightly lower than would be expected. The number contributing to KiwiSaver at the time of the survey was approximately 29 per cent of the New Zealand population (aged 18 and over; calculated from IRD statistics). KiwiSaver had started less than two years before the survey took place, and numbers were growing rapidly. Some respondents may not have reported their membership correctly, especially if they had been auto-enrolled. 
Table 7.9: Planning and financial knowledge characteristics of sample

\begin{tabular}{|l|c|}
\hline & $\begin{array}{c}\text { Sample, } \mathbf{n = 8 5 0} \\
\text { Weighted data }\end{array}$ \\
\cline { 2 - 2 } Retirement planning category & Per cent \\
\hline Contributing to KiwiSaver & \\
No or don't know & 75.9 \\
Yes & 24.1 \\
\hline Made a will & \\
No or don't know & 46.7 \\
Yes & 53.3 \\
\hline How much thought about financial & \\
planning for retirement? & \\
A little or not at all & 44.3 \\
A lot or a fair amount & 55.6 \\
Missing & 0.1 \\
\hline My life is determined by my own & \\
actions & \\
Strongly or somewhat agree & 93.9 \\
Neither agree or disagree & 3.4 \\
Somewhat or strongly disagree & 2.7 \\
\hline My life is determined by things & \\
beyond my control & \\
Strongly or somewhat agree & \\
Neither agree or disagree & \\
Somewhat or strongly disagree & \\
\hline My life is controlled by the actions & \\
of other people & \\
Strongly or somewhat agree & 30.1 \\
Neither agree or disagree & 32.6 \\
Somewhat or strongly disagree & \\
\hline Financial Knowledge group & \\
Low & \\
Middle & \\
High & \\
Lowest & \\
Advanced & \\
\hline Source: Analysis of Finang & \\
\hline
\end{tabular}

Source: Analysis of Financial Knowledge 2009 survey responses 


\subsection{Methods of analysis}

All statistical analysis was carried out using SPSS version 18. As variables were categorical, exploratory analysis searched for associations between responses and selected variables using Pearson's chi-squared tests. Significant standardised residuals within the contingency table were identified. For some tests of association, binary or multinomial logistic regression was then used with regression equation:

$$
P(\text { outcome })=\frac{1}{1+e^{-\left(b_{0}+b_{1} X_{1}+b_{2} X_{2}+\cdots\right)}}
$$

The $X_{i}$ input into the model were the potentially salient predictor variables identified in exploratory analysis, re-coded where 1 indicated the category as shown, and 0 all other responses. Both backward and forward stepwise methods were tested. Although inclusion would have been preferred as a possible error over exclusion, the fit of the model was generally better with the forward method.

SLS responses were compared to population indicators of expected lifespan from life tables. First, aggregate measures of survey responses to SLS were compared to benchmark aggregate population lifespan indicators. This checked whether the SLS were centralised around one norm, ignoring the distribution by age. This analysis is in section 8.1.1. 
Next, the gap between SLS and life table lifespan was calculated for each respondent to form a distribution for each life table used. This gap is a measure of respondents' underestimation of lifespan relative to that calculated from life expectancy e from the life table $\mathrm{T}$ for the respondent's age $\mathrm{x}$ and gender g:

$$
\text { Underestimation }(x, g, T)=x+e_{x, g, T}-S L S_{x, g}
$$

Calculating the gap for each age and gender improves on some previous studies as it allows for the characteristic of lifespan prospects improving as age increases and creates a distribution amenable to analysis. Four Statistics New Zealand life tables were used as the source for comparator population lifespans (T):

- Statistics New Zealand's latest period life table as at the time of the survey (2005-7);

- The cohort life table relevant to the time of the survey, that is, for all ages in calendar year 2009. This table was calculated and made available for this purpose by Statistics New Zealand on each of the three main scenarios for future mortality in the 2009-base population projections. The use of these cohort tables introduces benchmarks incorporating assumptions for future mortality improvement.

The importance of using population life tables as the benchmark - apart from the precedent of following previous studies - is that the tables are the official source of such information, and are used in domains which may provide indicators for individual expectation-setting, for example by policy makers. Thus the extent of underestimation is set in the policy context. The 
question is therefore not whether each individual lifespan expectation is accurate - that would be impossible to determine until each of the survey respondents had died - but rather the extent of the collective tendency for expectations to follow population benchmarks, taking into account the age and gender characteristics within the population. The summary measures of the distribution of the gap from each life table provide an overview of collective over- or underestimation of lifespan. Regression analyses profile the characteristics of those most likely to over- or underestimate. These analyses are in section 8.3.

SLS was collected at quinquennial ages, with the first group "60 or under" and the last "Over 100". These groups were assigned an SLS of 60 and 105 respectively where numerical calculations were necessary. Comparator lifespans from life tables were also rounded to multiples of 5 years to be consistent. Thus probabilities of survival between quinquennial SLS were not analysed, but this additional detail is not necessary to understand the extent of underestimation. The pattern of comparison between quinquennial SLS and quinquennial life table lifespans proved sufficiently clear for robust interpretation of results.

Age was not uniquely defined for either age of respondent or SLS. Age of respondent had been collected in 5 year age bands except for groups aged 1819, aged 65-74 and aged 75 and over. The variable of interest $x+e_{x}$ varies little across 5 year age bands, and as lifespans were rounded were rarely different for lower and upper ages of a band except for the oldest age group. The gap was taken to be zero, or SLS deemed "congruent" to the table value, if the SLS was within the range of life table lifespans from the lower and upper age of the 
age band; positive if SLS fell below the lifespan of the lower age and negative if SLS were above the lifespan of the upper age. This is a strong test for noncongruence, especially for those aged 75 and over.

\subsection{Limitations of this analysis}

This analysis necessarily had to use what was available from the Financial Knowledge survey. This was not a problem insofar as the wordings of the questions inserted on subjective life expectancy were as requested. However, the survey collected responses to other questions as decided by the steering group outside of considerations of this specific research. The data collected which formed the independent variables for this analysis were therefore not designed around the question of subjective lifespans. No obvious variable was missing for this analysis which focuses on subjective lifespan and retirement. If the intention had been to carry out an analysis of subjective lifespans in association with health factors then this survey would not have been the starting point.

Possible limitations of this analysis arise not from the availability of data in aggregate, but rather in the detail of three specific variables. The first, ethnicity has already been discussed. The sample size for ethnicities other than solely New Zealand European is smaller than ideal, as is the case for many surveys in New Zealand which are not supplemented by booster samples. Ethnic booster samples were available for the Financial Knowledge survey 2006, but budget constraints precluded their use for the 2009 survey used here. 
The second possible limitation is the grouping of SLS and retirement age responses to quinquennial years. While this was done to help the respondents answer more easily, it does risk losing potential responses between the multiples of five years. However, previous studies on subjective lifespans showed that bunching at quinquennial ages was likely to occur anyway which itself would have reduced the power of using single age data. Further, the analysis using SLS treated the quinquennial rounding appropriately, for example by comparing to rounded lifespans from life tables, and still sufficient data on the degree of over- and underestimation of lifespan was obtained. Therefore, the use of rounded SLS is not considered a significant limitation, although an unconstrained range of responses would be required to test whether that holds true.

However, it may be more of a limitation for responses to the question on likely retirement age. There is a smaller range of likely retirement ages given than SLS, and intuitively, it is perhaps more likely that an individual can imagine retiring at, say, 67 rather than 65 or 70 , compared to picking 87 as SLS instead of 85 or 90 . The analysis using retirement ages was therefore chosen to minimise distortion from the use of quinquennial ages for response. For example, the implied length of retirement was calculated as rounded SLS less rounded retirement age and then grouped into three categories, rather than analysing the variable of implied length of retirement as if it were linear.

The third limitation from the design of the survey is that age of respondent was collected in age bands. In particular the oldest age bands of 65-74 and 75+ were wider than the five year bands used at younger ages. This is a common complaint of any research on later life issues using survey 
evidence as it limits the information available just as heterogeneity of many variables associated with ageing increases. For example, 75-year-olds would be expected to have a very different average SLS than 95-year-olds. The effect on the SLS analysis of using age bands rather than single years for age of respondents is mitigated somewhat by the use of lifespans $(x+e x)$ rather than life expectancy (ex) as the dependent variable of interest. Lifespans vary little across most age bands. For example, in the 2005-7 male period life table, lifespan rounded to the nearest multiple of five is 85 years for all ages from 63 to 78 . It is then 90 years until age 88 , reaching 100 years at age 95 . The most critical impact on this analysis of not having age of respondent by single age is therefore in the age band 75+. In the absence of any data on the actual age of the oldest survey respondent, age 89 was used as the end point of this last age band. Sensitivity testing on this assumption is discussed in the text where relevant. The interpretation of these results is not affected. Potential exploration of how SLS features in the financial planning of over 75 year olds, for example for annuity purchase, is limited, but this study is concerned with longevity risk in the pre-retirement period.

Finally, this is a survey at one point in time of New Zealanders' SLS, and subsequent changes in SLS may have occurred. After this survey was carried out in 2009, the earthquakes in Christchurch of 2010 and 2011 may have heightened Cantabrians' sense of mortality. The subsequent media coverage of the risks from natural hazards in other parts of New Zealand may also have affected New Zealanders' perceptions of mortality. This is despite the number of earthquake deaths being fewer than one per cent of the total annual deaths in New Zealand. Smith (2008) suggests that those affected by a natural hazard may reduce their lifespan expectations. If so, the extent of underestimation of 
lifespans in New Zealand may be greater than shown by this 2009 survey, but the effect may be temporary.

The limitations are judged not to undermine the results of this analysis, but suggest points of difference for future surveys on longevity expectations.

\subsection{Conclusions}

Subjective longevity expectations are an important element of policy and individuals' own retirement planning, but there has been until recently no data on longevity expectations in New Zealand, and few large sample surveys on the subject internationally.

The international studies provide somewhat inconclusive evidence, but some common themes emerge together with some unanswered research questions. Summary findings reporting subjective lifespans congruent to population life tables usually hide both over- and underestimation of longevity, but the general tendency appears more to be underestimation. People may have a broad view of average longevity, or they may take some individual mortality risk factors into account which can introduce additional underestimation. A shared theme is that men reflect that they have lower actual longevity than women, but are more optimistic, so that women are more likely to underestimate their actual longevity. However, there is almost no data on how people think about longevity, if they think about it at all, and why they choose a particular estimate of their own likely longevity when asked.

A reasonable hypothesis consistent with the limited evidence is that people are aware of current information on longevity, both the average for the 
population and some factors causing it to vary for individuals with higher or lower than average mortality risk. The underestimation of individual longevity may occur if available public information on longevity is itself outdated, or does not help individuals to understand the potential for and implications of mortality improvement and the impact of individual risk factors.

The New Zealand study here has been designed to test this hypothesis and fill research gaps on the extent of any collective underestimation of lifespan in the population, by whom and why. The new survey extends the approach used in the international studies in three important ways. First, additional variables for analysis include retirement planning and financial knowledge to explore longevity risk in the context of retirement planning. Second, new questions and risk factors probe the rationale for choice of subjective lifespan. Third, the analysis enables the gaps to potential lifespan to be estimated allowing for age and gender, and tested against population life tables that allow for improving mortality on different assumptions. 


\section{Chapter 8: New Zealanders' lifespan expectations}

The findings of the survey on New Zealanders' lifespan expectations are structured in sections of this chapter based on three out of the four research gaps identified from international studies: what lifespan New Zealanders expect; how those lifespan expectations are formed; and, what they imply for the profile of lifespan underestimation in New Zealand. A final section discusses the conclusions that can be drawn from this survey evidence. The fourth research gap, on how subjective lifespan expectations are related to retirement planning, is considered in the next chapter.

\subsection{New Zealanders' subjective lifespan expectations (SLS)}

The survey asked "What age do you think you will live to?" to obtain a subjective lifespan (SLS). This section first describes the distribution of SLS responses and then investigates the profile of respondents and nonrespondents.

\subsubsection{Distribution of SLS}

The shape of the distributions of SLS given by men and women were very different (Figure 8.1). The distribution of female SLS had a clear mode at age 85. The distribution of the male SLS was weighted more to the left, with a flat peak from age 75 to 85 . There were higher proportions of men than women in both the lowest and highest SLS groups. 
Figure 8.1: What age do you think you will live to? Subjective lifespan, estimated percentage of the population by gender

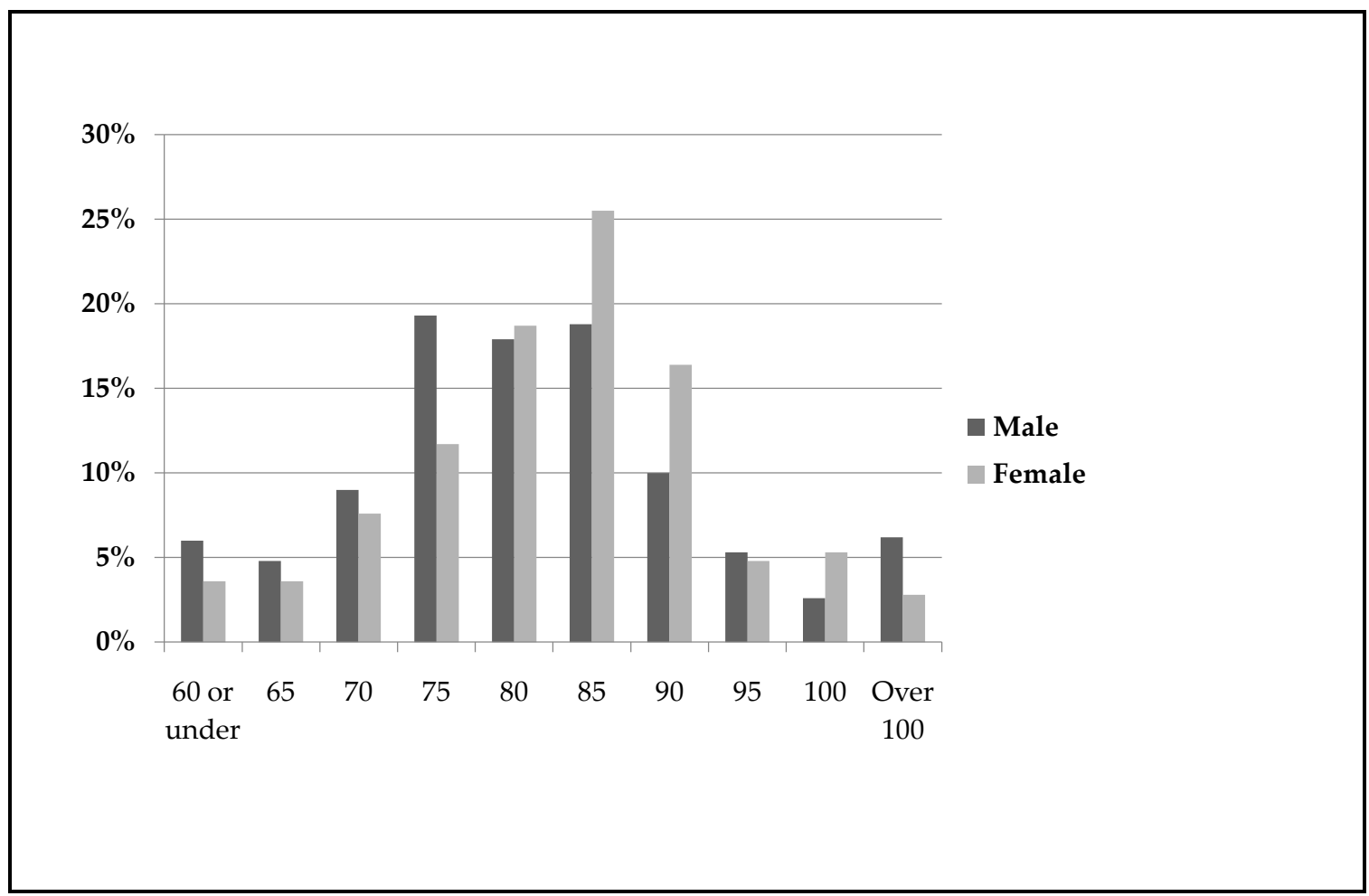

Source: Analysis of Financial Knowledge 2009 survey responses. $\mathrm{n}=365$ male, 361 female who gave an SLS

To explore whether the population takes cues from contemporary ageindependent indicators of population lifespan, Table 8.1 sets out measures of average SLS alongside such benchmarks. Life expectancy at birth is the most obvious candidate for a mean age-independent published indicator to test. The latest two Statistics New Zealand period life tables are used as comparators. Further age-independent indicators used are derived from the lifespans that people would see around them. Statistics New Zealand provides ages at death in New Zealand, from which the mean, median and mode have been extracted. 
Table 8.1: Summary of sample SLS compared to age-independent population lifespan indicators

\begin{tabular}{|l|c|c|c|}
\hline \multirow{2}{*}{} & \multicolumn{3}{|c|}{$\begin{array}{c}\text { SLS, actual or life table } \\
\text { measure, in years }\end{array}$} \\
\cline { 2 - 4 } & Total & Men & Women \\
\hline Sample distribution SLS & 726 & 365 & 361 \\
N (weighted) & & & \\
Mean & 81.9 & 81.0 & 82.8 \\
Median & 80 & 80 & 85 \\
Mode & 85 & 75 & 85 \\
Mean life expectancy at birth & & & \\
Complete period life table, 2005-7 & - & 78.0 & 82.2 \\
Provisional abridged life table 2007-9 & - & 78.4 & 82.4 \\
& & & \\
\hline Actual age of deaths in New & & & \\
Zealand of New Zealand residents, & & & \\
2009 & 74.3 & 71.5 & 77.2 \\
Mean & 79.8 & 76.9 & 82.8 \\
Median & 83 & 83 & 89 \\
Mode & & & \\
\hline
\end{tabular}

Source: Analysis of Financial Knowledge 2009 survey responses and Statistics New Zealand data.

The mean age at death from the life expectancy at birth measure is close to the mean SLS for women, but not for men. Mean actual ages at death are lower than period table life expectancy at birth as the latter is the mean age at death for a hypothetical population affected less by deaths at young ages. However, for the total population, the median SLS of 80 years and the mode of 85 years are close to those measures for actual deaths in New Zealand. Moreover, there is indeed a close similarity between the shape of the distribution of actual deaths within the age range of SLS survey responses and the distribution of SLS (Figure 8.2). The peaks and the asymmetry of the SLS distributions of both men and women align with those of the distributions of 
actual deaths, although the peak in male SLS at 75 and female at 85 are more pronounced than in actual deaths. A further inconsistency is the higher proportion of men compared to women with an SLS of 100 or more when reality is reversed. However, the general pattern suggests that, collectively, the population takes cues for SLS from the contemporary pattern of deaths in the population.

Figure 8.2: Distribution of SLS responses compared to actual deaths in New

\section{Zealand in 2009}

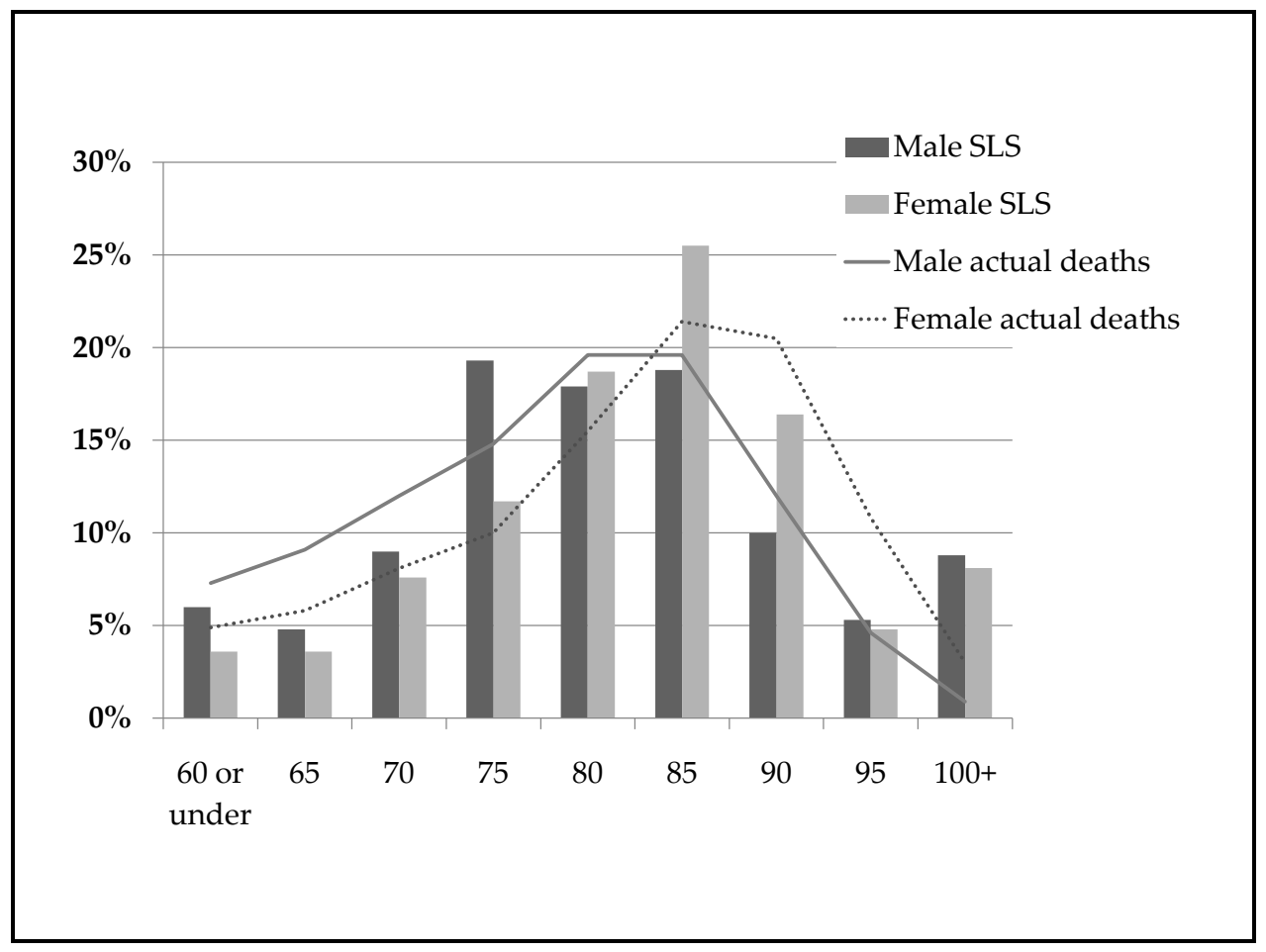

Source: SLS data from Financial Knowledge 2009 survey responses. n=365 male, 361 female who gave an SLS. Actual deaths 2009, 100 per cent = deaths over age 57. Actual deaths at ages 100 and over grouped in data source from Statistics New Zealand.

When aggregate measures of SLS are compared to population benchmarks which do not vary by age, an important actuarial property is ignored; that life expectancy ex decreases with each additional year of age $(x)$ by less than one 
year because those that survive the extra year are on average less frail than the group would have been with the addition of those that have died. Life expectancy at birth would therefore be lower than mean likely lifespans for an adult population sample. This is also true for central measures of the distribution of actual deaths in a past year. As mortality is improving, the central measures of age at death for an ageing population would be expected to increase every year. If survey respondents were anticipating these effects then the sample SLS would be expected to exceed these time-dependent indicators. This is why a more insightful analysis is provided by examining the distribution of the gap between SLS for each respondent and gender-, ageand time-dependent population lifespan measures. Section 8.3 shows the results of this analysis.

\subsubsection{Profile of SLS respondents}

Most survey respondents, around 85 per cent, gave a subjective lifespan when asked what age they thought they would live to. Fewer than 10 respondents refused to give an answer; 116 said they didn't know (13.7 per cent of sample, weighted estimate, sample error $+/-2.3$ per cent). This is within the range of previous international SLS surveys, but the "don't knows" form a sizeable group worth profiling. For an exploratory profiling of responses to the SLS question, the sample was split into three groups: those choosing an SLS up to and including the median of 80 years; those choosing an SLS of 85 years and above (responses were collected at quinquennial ages) and those saying don't know or refused. Results are in Table 8.2. 
Gender and age are significantly associated with not giving an SLS. Women are significantly more likely than men to say they don't know when asked for their SLS. Women form two-thirds of the "don't knows". Further, those under 30 are not more or less likely to say "don't know" to the SLS question, but after age 30, the likelihood of saying "don't know" increases with age. It would be speculative to say whether uncertainty comes with age, or whether today's older cohort is less confident at giving SLS than the younger cohort.

Those saying "don't know" are more likely to have basic or secondary education only; be in the Low financial knowledge group; and, disagree that life can be determined by own actions. Financial planning for retirement is not associated, but contributing to KiwiSaver is, with KiwiSaver contributors less likely to say "don't know" when asked for SLS. Ethnicity and comfort with English are significantly associated, with Pacific Peoples being more likely to say "don't know". In summary, respondents to the SLS question are more likely to be male, middle aged rather than under 30 or over 60 , well educated (which may mean financially better off), knowledgeable about financial issues, and confident about influencing their life themselves. 
Table 8.2: What age do you think you will live to?

\begin{tabular}{|c|c|c|c|}
\hline \multirow[b]{2}{*}{ Variable/category } & \multicolumn{3}{|c|}{ Percentage within category } \\
\hline & $\begin{array}{l}\text { SLS } 80 \\
\text { (median) } \\
\text { and below }\end{array}$ & $\begin{array}{l}\text { SLS } 85 \text { and } \\
\text { above }\end{array}$ & $\begin{array}{l}\text { Don't know } \\
\text { or refused }\end{array}$ \\
\hline Total sample, $n=850$ & 43.6 & 41.8 & 14.6 \\
\hline \multicolumn{4}{|l|}{ Gender ${ }^{* * *}$} \\
\hline Male & $51.1^{*}$ & 38.6 & $10.3^{*}$ \\
\hline Female & $36.9^{*}$ & 44.8 & $18.3^{*}$ \\
\hline \multicolumn{4}{|l|}{ Age } \\
\hline Under 30 & 50.3 & 38.0 & 11.7 \\
\hline 30 and over & 42.1 & 42.7 & 15.2 \\
\hline \multicolumn{4}{|l|}{ Age 30 and over $* * *$} \\
\hline $30-44$ & 47.7 & 42.2 & $10.2^{*}$ \\
\hline $45-59$ & 48.0 & 38.1 & 13.9 \\
\hline $60+$ & $27.5^{* *}$ & 48.7 & $23.8^{* *}$ \\
\hline \multicolumn{4}{|l|}{ Ethnicity (single count) $* *$} \\
\hline New Zealand European & 40.6 & 44.4 & 15.0 \\
\hline Māori & 55.2 & 28.4 & 16.4 \\
\hline NZ European and Māori & 55.3 & 42.1 & 2.6 \\
\hline Pacific & 31.4 & 40.0 & $28.6^{*}$ \\
\hline Asian & 55.8 & 28.6 & 15.6 \\
\hline All other & 43.8 & 48.8 & 7.5 \\
\hline \multicolumn{4}{|l|}{ Place of birth } \\
\hline Born in New Zealand & 44.3 & 41.7 & 14.0 \\
\hline Born outside New Zealand & 42.1 & 41.7 & 16.2 \\
\hline \multicolumn{4}{|l|}{ Comfort with English ** } \\
\hline Very & 43.7 & 42.8 & 13.5 \\
\hline Somewhat & 52.1 & 27.1 & 20.8 \\
\hline Not very or not at all & 18.8 & 37.5 & $43.8^{* *}$ \\
\hline \multicolumn{4}{|l|}{ Education $* * *$} \\
\hline Primary or basic secondary & 39.3 & 39.9 & $20.8^{*}$ \\
\hline Secondary school & 37.8 & 41.3 & $20.9^{*}$ \\
\hline Tertiary or postgraduate & 48.0 & 42.6 & $9.4^{* *}$ \\
\hline \multicolumn{4}{|l|}{ Personal income $* * *$} \\
\hline Up to and including $\$ 30,000 \mathrm{pa}$ & 41.4 & 42.6 & 16.0 \\
\hline Over $\$ 30,000$ pa & 49.2 & 42.0 & $8.8^{* *}$ \\
\hline Don't know or refused & 31.4 & 36.0 & $32.6^{* * *}$ \\
\hline \multicolumn{4}{|l|}{ Net wealth } \\
\hline Negative & 47.4 & 35.9 & 16.7 \\
\hline 0-\$100k & 44.8 & 44.2 & 11.0 \\
\hline$\$ 101 \mathrm{k}-\$ 300 \mathrm{k}$ & 43.6 & 39.1 & 17.3 \\
\hline$\$ 301 k-\$ 600 k$ & 39.8 & 43.1 & 17.1 \\
\hline$\$ 601 \mathrm{k}+$ & 41.7 & 55.1 & 9.1 \\
\hline
\end{tabular}


Table 8.2: What age do you think you will live to?, continued

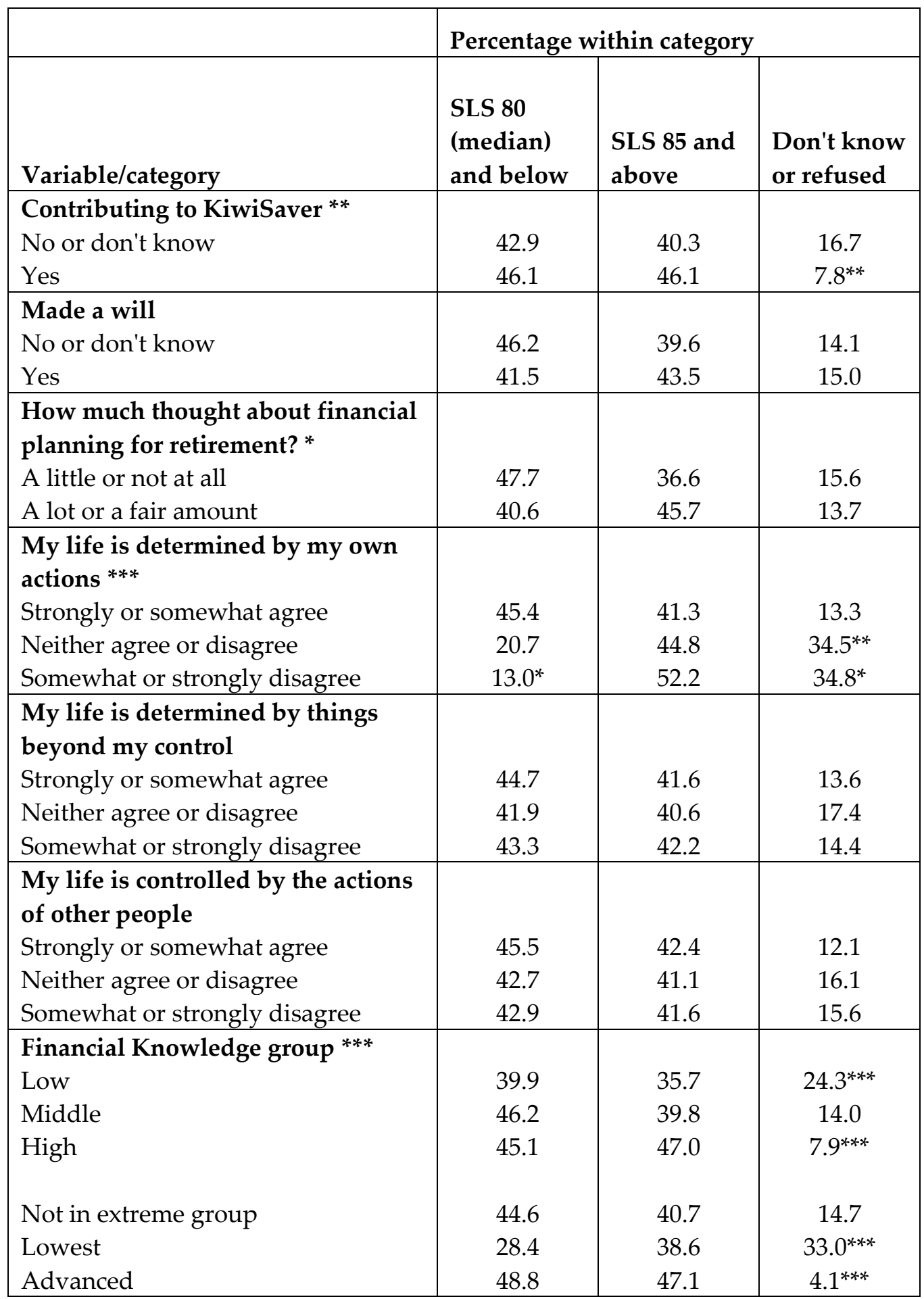

Source: Analysis of Financial Knowledge 2009 survey responses.

Note: A Pearson's chi-squared test was carried out between the variable denoted in the table columns and each variable in a subsection of the table. An asterisk next to the variable name denotes significant association between the two variables. An asterisk within a cell denotes a high standardised residual value for that category under the assumption of no association between the two variables. ${ }^{*} \mathrm{p}<.05 ;{ }^{* *} \mathrm{p}<.01$; ${ }^{* * *} \mathrm{p}<.001$. 
These findings are consistent with international evidence. The only previous study to study the "don't knows" found that the likelihood of saying "don't know" to the SLS question increased with age, particularly for women, and decreased with education and a sense of control over one's own life (Mirowsky 1999 p. 974-5). Further, international studies have consistently found that men are more likely to overestimate likely longevity than women (Hurd and McGarry 1995, 2002; Mirowsky 1999; O'Brien et al. 2005). This analysis gives a possible explanation, which also fits with the higher proportion of men than women with SLS of 100 or more: those men who give a response to the SLS question are more likely to be guessing than the female respondents, and they tend to guess with some bravado towards the higher SLS.

\subsection{How lifespan expectations are formed}

This section reports on the results of the New Zealand survey questions that aimed to shed more light on how responses to questions of lifespan expectations are formed.

\subsubsection{How much New Zealanders have thought about lifespan}

Potential lifespan seems to have been considered by a majority of the population. An estimated 70 per cent of adult New Zealanders (69.5 per cent of sample, weighted estimate, sample error +/-3.1 per cent) claim to have given the age they might live to some or a lot of thought (Figure 8.3). The 30 per cent who say they have not or never thought about SLS are significantly more likely to say they don't know their SLS $(\mathrm{p}<.001)$. Yet only 15 per cent say they 
do not know what their SLS is. This means that over 20 per cent (15/70) of SLS responses to the survey could be a guess from people thinking about it for the first time.

Figure 8.3: How much thought given to age might live to, estimated percentage of the population

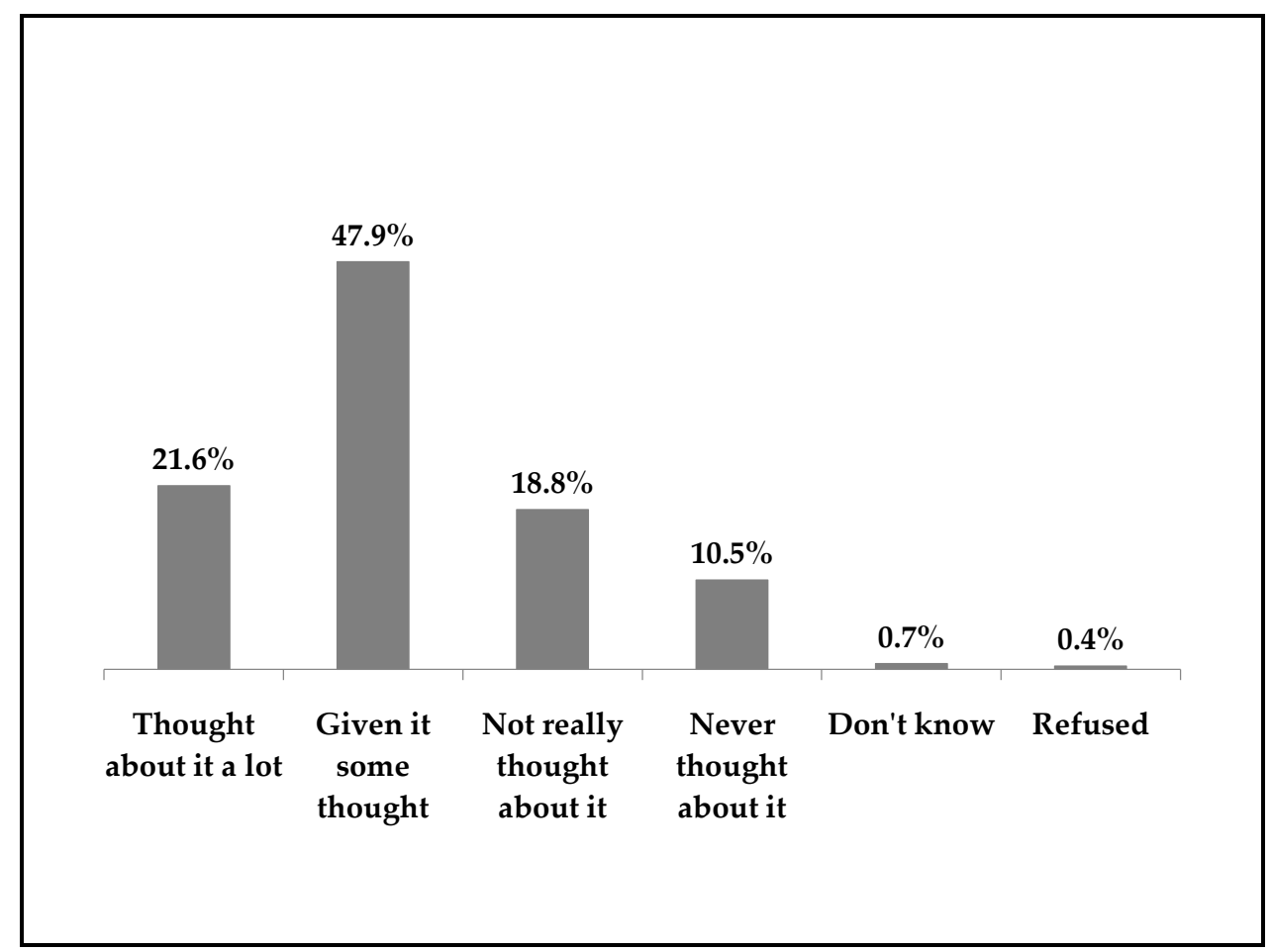

Source: Analysis of Financial Knowledge 2009 survey responses. $\mathrm{n}=850$.

Having thought about SLS was strongly associated with age. Specifically, those aged under 30 were significantly more likely to say they had never or not really thought about the age they might live to $(\mathrm{p}<.001)$ than those aged 30 or over. Figure 8.4 shows that those aged 18-29 were roughly equally likely to say they had or hadn't given the age they might live to some thought; whereas around three-quarters of those aged 30 and over said they had given it some thought. The odds of a New Zealander aged 30 and over having given 
the age they might live to some or a lot of thought were 2.5 times higher than the under 30s.

Figure 8.4: What thought given to age might live to, estimated percentage of the population by age group

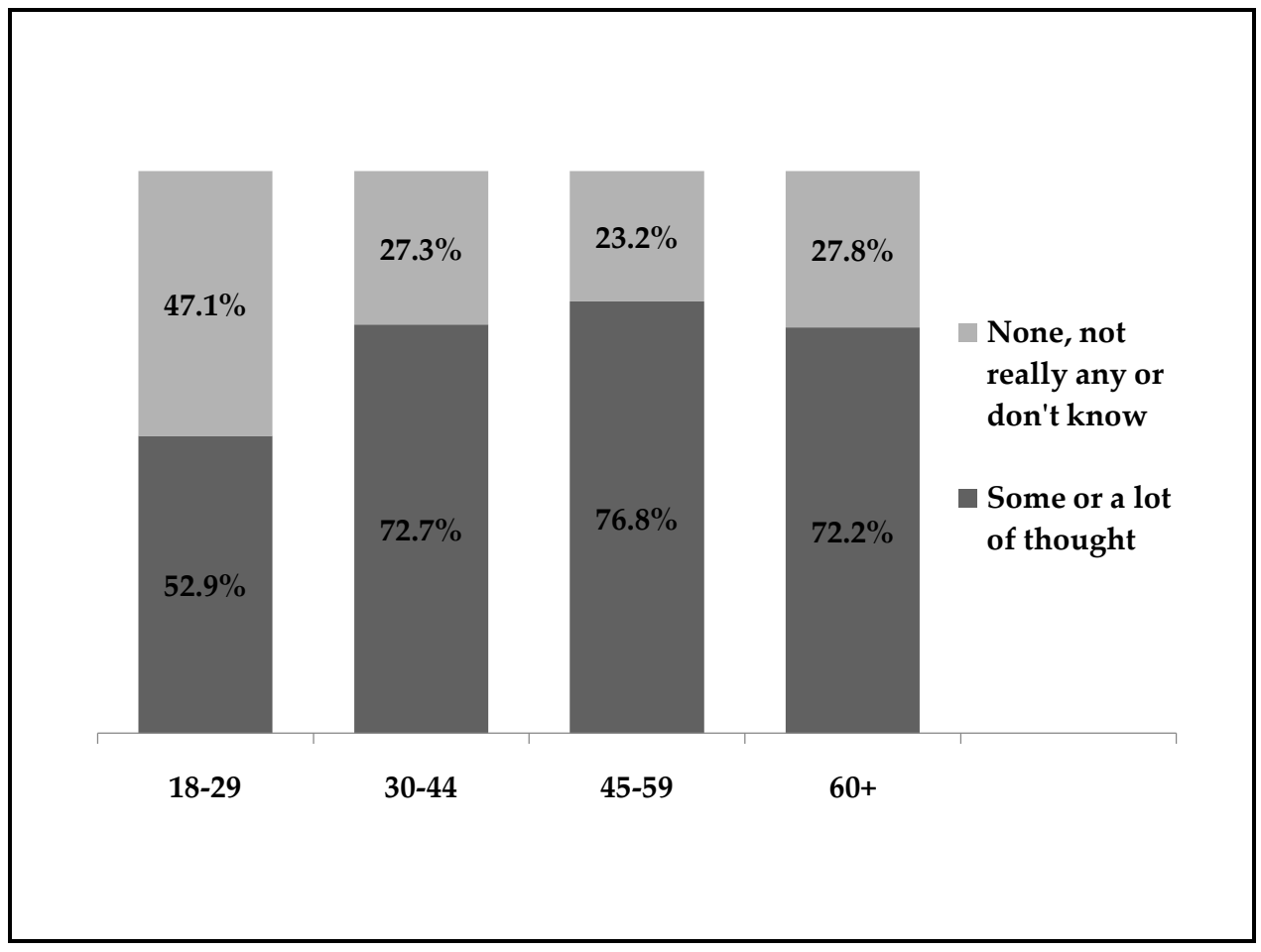

Source: Analysis of Financial Knowledge 2009 survey responses. $\mathrm{n}=850$.

The two subsamples of respondents aged 18-29 and aged 30 and over were investigated for associations with degree of having thought about SLS. Within those aged under 30, no significant associations for having thought about age might live to were identified (analysis not shown). Table 8.3 shows the results of this analysis for the 30 and over age group. The responses "never" or "not really thought" or don't know were grouped, as were "some" or "a lot of thought". 
Table 8.3: Have you thought about the age you might live to?

\begin{tabular}{|c|c|c|}
\hline Age 30 and over & Percentage & thin category \\
\hline Variable/category & $\begin{array}{l}\text { No, not really } \\
\text { or don't know }\end{array}$ & $\begin{array}{l}\text { Some or a lot } \\
\text { of thought }\end{array}$ \\
\hline Total aged 30 and over, $n=671$ & 26.1 & 73.9 \\
\hline $\begin{array}{l}\text { Gender } \\
\text { Male } \\
\text { Female }\end{array}$ & $\begin{array}{l}25.4 \\
26.8\end{array}$ & $\begin{array}{l}74.6 \\
73.2\end{array}$ \\
\hline $\begin{array}{l}\text { Age } \\
30-44 \\
45-59 \\
60+\end{array}$ & $\begin{array}{l}27.2 \\
23.3 \\
28.0\end{array}$ & $\begin{array}{l}72.8 \\
76.7 \\
72.0\end{array}$ \\
\hline $\begin{array}{l}\text { Ethnicity (single count) } * * * \\
\text { New Zealand European } \\
\text { Māori } \\
\text { NZ European and Māori } \\
\text { Pacific } \\
\text { Asian } \\
\text { All other }\end{array}$ & $\begin{array}{c}23.0 \\
47.9^{* *} \\
8.0 \\
42.9 \\
32.1 \\
27.9\end{array}$ & $\begin{array}{l}77.0 \\
52.1 \\
92.0 \\
57.1 \\
67.9 \\
72.1\end{array}$ \\
\hline $\begin{array}{l}\text { Place of birth } \\
\text { Born in New Zealand } \\
\text { Born outside New Zealand }\end{array}$ & $\begin{array}{l}24.9 \\
29.3\end{array}$ & $\begin{array}{l}75.1 \\
70.7\end{array}$ \\
\hline $\begin{array}{l}\text { Comfort with English *** } \\
\text { Very } \\
\text { Somewhat } \\
\text { Not very or not at all }\end{array}$ & $\begin{array}{l}24.2 \\
51.7^{* *} \\
77.8^{* *}\end{array}$ & $\begin{array}{l}75.8 \\
48.3 \\
22.2\end{array}$ \\
\hline $\begin{array}{l}\text { Education } * * * \\
\text { Primary or basic secondary } \\
\text { Secondary school } \\
\text { Tertiary or postgraduate }\end{array}$ & $\begin{array}{l}39.2^{* *} \\
24.0 \\
21.8\end{array}$ & $\begin{array}{l}60.8 \\
76.0 \\
78.2\end{array}$ \\
\hline $\begin{array}{l}\text { Personal income } * * * \\
\text { Up to and including } \$ 30,000 \text { pa } \\
\text { Over } \$ 30,000 \text { pa } \\
\text { Don't know or refused }\end{array}$ & $\begin{array}{l}29.0 \\
20.1^{*} \\
41.4^{*}\end{array}$ & $\begin{array}{l}71.0 \\
79.9 \\
58.6\end{array}$ \\
\hline $\begin{array}{l}\text { Net wealth } \\
\text { Negative } \\
0-\$ 100 \mathrm{k} \\
\$ 101 \mathrm{k}-\$ 300 \mathrm{k} \\
\$ 301 \mathrm{k}-\$ 600 \mathrm{k} \\
\$ 601 \mathrm{k}+\end{array}$ & $\begin{array}{l}28.8 \\
35.0 \\
27.0 \\
23.0 \\
20.5\end{array}$ & $\begin{array}{l}72.7 \\
65.0 \\
73.0 \\
77.0 \\
79.5\end{array}$ \\
\hline
\end{tabular}


Table 8.3: Have you thought about the age you might live to?, continued

\begin{tabular}{|c|c|c|}
\hline \multirow{2}{*}{$\begin{array}{l}\text { Age } 30 \text { and over } \\
\text { Variable/category }\end{array}$} & \multicolumn{2}{|c|}{ Percentage within category } \\
\hline & $\begin{array}{l}\text { No, not really } \\
\text { or don't know }\end{array}$ & $\begin{array}{c}\text { Some or a lot of } \\
\text { thought }\end{array}$ \\
\hline $\begin{array}{l}\text { Contributing to KiwiSaver } \\
\text { No or don't know } \\
\text { Yes }\end{array}$ & $\begin{array}{l}26.1 \\
26.0\end{array}$ & $\begin{array}{l}73.9 \\
74.0\end{array}$ \\
\hline $\begin{array}{l}\text { Made a will } * * \\
\text { No or don't know } \\
\text { Yes }\end{array}$ & $\begin{array}{l}32.2 \\
22.7 \\
\end{array}$ & $\begin{array}{l}67.8 \\
77.3\end{array}$ \\
\hline $\begin{array}{l}\text { How much thought about financial } \\
\text { planning for retirement? } * * * \\
\text { A little or not at all } \\
\text { A lot or a fair amount }\end{array}$ & $\begin{array}{c}37.8^{* * *} \\
19.9^{*} \\
\end{array}$ & $\begin{array}{c}62.2^{*} \\
80.1 \\
\end{array}$ \\
\hline $\begin{array}{l}\text { My life is determined by my own } \\
\text { actions } \\
\text { Strongly or somewhat agree } \\
\text { Neither agree or disagree } \\
\text { Somewhat or strongly disagree }\end{array}$ & $\begin{array}{c}26.6 \\
5.9 \\
25.0\end{array}$ & $\begin{array}{l}73.4 \\
94.1 \\
75.0\end{array}$ \\
\hline $\begin{array}{l}\text { My life is determined by things } \\
\text { beyond my control } \\
\text { Strongly or somewhat agree } \\
\text { Neither agree or disagree } \\
\text { Somewhat or strongly disagree }\end{array}$ & $\begin{array}{l}23.0 \\
34.7 \\
27.0\end{array}$ & $\begin{array}{l}77.0 \\
65.3 \\
73.0 \\
\end{array}$ \\
\hline $\begin{array}{l}\text { My life is controlled by the actions } \\
\text { of other people } \\
\text { Strongly or somewhat agree } \\
\text { Neither agree or disagree } \\
\text { Somewhat or strongly disagree }\end{array}$ & $\begin{array}{c}15.7^{* *} \\
23.7 \\
33.1^{* *}\end{array}$ & $\begin{array}{l}84.3 \\
76.3 \\
66.9\end{array}$ \\
\hline $\begin{array}{l}\text { Financial Knowledge group } * * * \\
\text { Low } \\
\text { Middle } \\
\text { High }\end{array}$ & $\begin{array}{l}38.0^{* *} \\
26.1 \\
20.1^{*}\end{array}$ & $\begin{array}{l}62.0 \\
73.9 \\
79.9\end{array}$ \\
\hline $\begin{array}{l}\text { Not in extreme group } \\
\text { Lowest } \\
\text { Advanced }\end{array}$ & $\begin{array}{c}27.2 \\
47.8^{* *} \\
16.2^{*}\end{array}$ & $\begin{array}{l}72.8 \\
52.2 \\
83.8\end{array}$ \\
\hline
\end{tabular}

Source: Analysis of Financial Knowledge 2009 survey responses.

Note: A Pearson's chi-squared test was carried out between the variable denoted in the table columns and each variable in a subsection of the table. An asterisk next to the variable name denotes significant association between the two variables. An asterisk within a cell denotes a high standardised residual value for that category under the assumption of no association between the two variables. ${ }^{*} \mathrm{p}<.05 ;{ }^{* *} \mathrm{p}<.01 ;{ }^{* * *} \mathrm{p}<.001$. 
Among those aged 30 and over, there is a significant association with being more likely to say that they have not thought about the age they will live to for: Māori, those less than very comfortable with English, those who have not thought about financial planning for retirement, those at the lowest levels of education and financial knowledge and those who agree that their life is controlled by other people. Those with higher incomes are significantly less likely to say they have not thought about the age they might live to, whereas those who did not know their income or refused to say are more likely to say they have not thought about it.

The general picture of the New Zealand population, then, is that having thought about personal longevity by age 30 is equally likely as not. Thereafter, more thought is likely to be given to SLS especially by those with better incomes or good financial knowledge, perhaps in tandem with financial planning for retirement. However, around a quarter of New Zealanders aged 30 and over still has not really thought about their likely lifespan.

The strong association with age uncovered here confirms that the international studies with older age samples, such as HRS and ELSA, or with younger age samples, such as the studies in psychology often carried out with small groups of students, cannot be used without question as guides to the profile of SLS and correlates for a population of all adult ages. This is particularly concerning because of the number of interpretive studies based on HRS. Further, as Māori are less likely to have thought about SLS, generalising the study of any international population study on SLS to New Zealand is questionable. 


\subsubsection{How New Zealanders choose their SLS}

Asking why survey participants chose their SLS was considered to be an integral part of this study. There was no theory from international studies to direct enquiry, and yet it was hypothesised that how New Zealanders think about their lifespan may influence not only whether SLS was high or low, but also how messages about population average lifespans or lifespan improvement may be received.

After giving an SLS, survey respondents were asked "For what reasons do you say that", and the unprompted answers coded and recorded, separately for first reason and subsequent responses. There were 35 codes used to collect reasons, so some grouping into manageable categories was necessary. The responses ranged from none to 8 reasons given (Figure 8.5). For 30 per cent of the sample (weighted) only one reason was given. The modal number of reasons was just one; the median two reasons and the mean 2.1. In over onethird of cases where two reasons were given, both reasons were the same according to the grouping developed, and explained below. Thus, the first reason given is likely to be a good indicator of why people gave the SLS they did. Analysis here concentrates on this 'main' reason only, grouped into five categories, described in the following. 
Figure 8.5: Number of reasons for why chose age will live to, estimated percentage of population

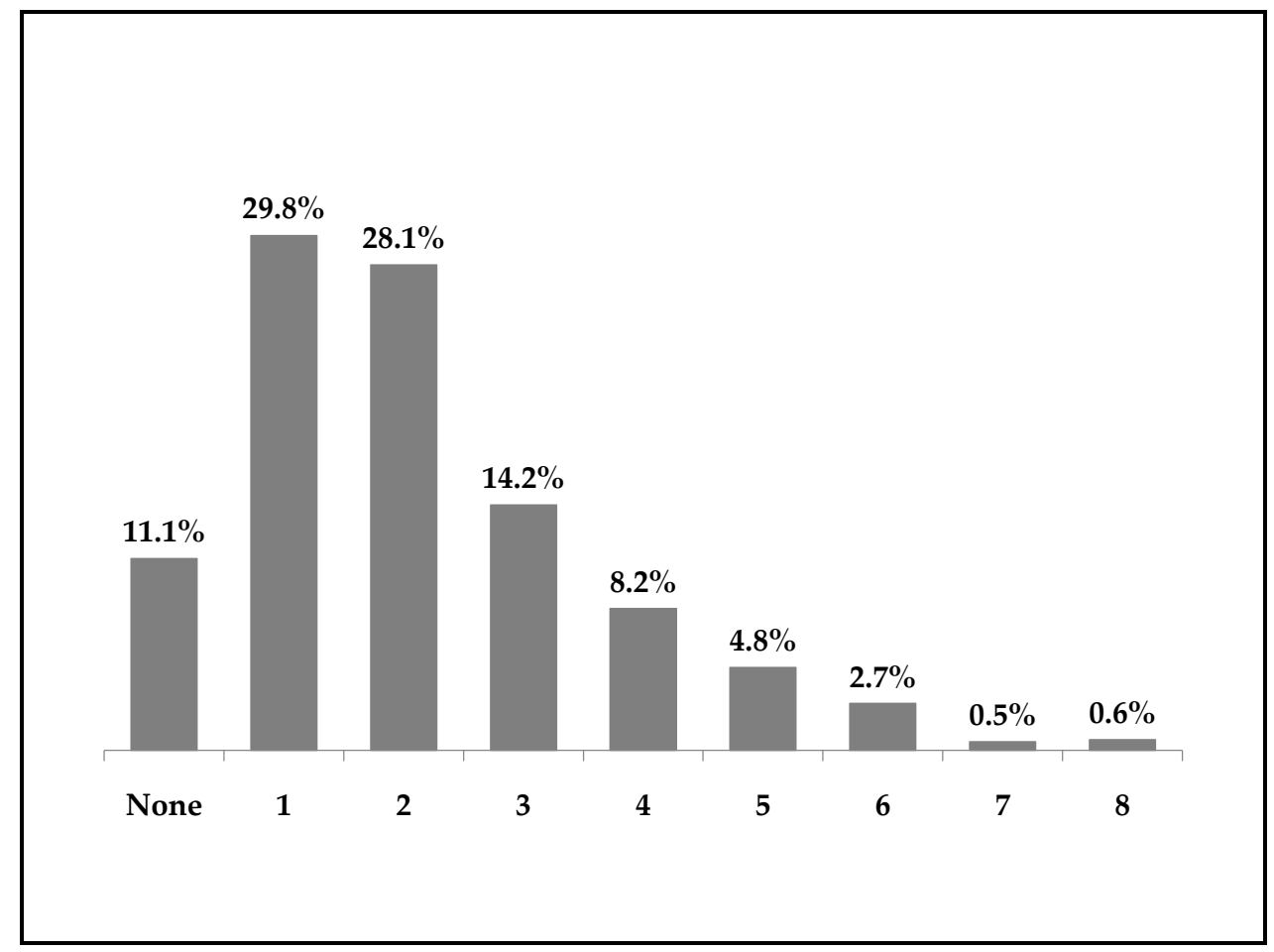

Source: Analysis of Financial Knowledge 2009 survey responses. $\mathrm{n}=850$.

\section{Distribution of main reason}

The first reasons given for choice of SLS were grouped into five categories as follows:

- Genes: Respondents who gave the reasons coded as "genetics", "thinking about my parents/grandparents" or "family history" formed the largest group, comprising an estimated 34 per cent of the population. Within the groups, nearly half mentioned their parents, a third "genetics", and under one-fifth their grandparents.

- Other or don't know: The long tail of other reasons coded was grouped with those who said "don't know" as some of those reasons were tantamount to saying don't know, for example, "just a number in my 
head", "it's out of my hands" or a wish such as "I want to be around for my grandchildren", or "that's a reasonable age". This group comprised an estimated 30 per cent of the population.

- Average/normal: Responses were coded as "That about average/normal (for someone like me)" or "thinking about people my age/that's about normal for people my age." This group comprised an estimated 10 per cent of the population.

- Good risk factors: A variety of lifestyle, but not genetic, reasons were coded as factors which indicated good health behaviour or favourable mortality risk, such as "I'm healthy" (9.9 per cent of first reasons), "I'm fit" (2.3 per cent) or "I don't smoke" (2.2 per cent). In total, this group comprised an estimated 17 per cent of the population.

- Bad risk factors: Similarly, lifestyle, but not genetic, reasons indicative of poor health or mortality risk were coded such as "I smoke" (3.5 per cent of first reasons) or "I've had health scares" (2.0 per cent). This group was just over half the size of the good risk factor group, at an estimated 9 per cent of the population.

SLS is external to personal behaviour or manageable risk for respondents in the first three groups. Their rationale is based on family history or a genetic explanation; they do not have an explanation or evade one by saying something like "it's out of my hands"; or, can only reason on the basis of their SLS being 'average' or 'normal'. These groups together make up around three quarters of adult New Zealanders. The remaining quarter of the New Zealand adult population gives personal risk factors - either positive or negative - as their first reason for choosing their SLS. 


\section{Profiling who gives each main reason}

Predictive variables for being in each of the five groups were identified by multinomial regression, following exploratory analysis with Pearson's chisquared tests and binary regression for each main reason (not shown). Results are in Table 8.4.

Those in the Low financial knowledge group have significantly greater odds of saying "other or don't know" or not having a reason for choosing a particular SLS relative to all other reasons. There is also a suggestion of a weaker association with having negative net wealth and saying "other or don't know". Giving as main reason "average/normal" has greater odds for those aged 30-44, with no other significant associations found.

Compared with saying "other or don't know" or not having a reason for choosing a particular SLS, the odds of giving genes as the main reason are greater for those in the High financial knowledge group as opposed to in the Low or Medium groups, those who gave New Zealand European as sole ethnicity as opposed to all other ethnicities, and those who have given their life expectancy some or a lot of thought rather than not really given it any or no thought. 
Table 8.4: Predictors of main reason for choice of SLS: multinomial logistic regression

\begin{tabular}{|c|c|c|c|c|c|}
\hline \multirow{3}{*}{$\begin{array}{l}\text { Each reason relative to } \\
\text { reference category "Other } \\
\text { or don't know" }\end{array}$} & \multirow{2}{*}{\multicolumn{2}{|c|}{ Coefficient }} & \multicolumn{3}{|c|}{ 95\% CI for Odds Ratio } \\
\hline & & & \multirow[b]{2}{*}{ Lower } & \multirow{2}{*}{$\begin{array}{l}\text { Odds } \\
\text { Ratio }\end{array}$} & \multirow[b]{2}{*}{ Upper } \\
\hline & $\mathbf{b}$ & SE & & & \\
\hline \multicolumn{6}{|l|}{ Genes } \\
\hline Constant & -0.11 & 0.39 & & & \\
\hline Age $30-44$ & -0.37 & 0.21 & 0.46 & 0.69 & 1.04 \\
\hline Have thought about SLS & $0.52^{*}$ & 0.20 & 1.13 & 1.68 & 2.51 \\
\hline Low financial knowledge & $-0.86^{* *}$ & 0.26 & 0.26 & 0.42 & 0.70 \\
\hline Female & 0.25 & 0.19 & 0.89 & 1.29 & 1.86 \\
\hline NZ Euro sole ethnicity & $0.42^{*}$ & 0.21 & 1.02 & 1.53 & 2.29 \\
\hline High financial knowledge & $0.80^{* *}$ & 0.24 & 1.40 & 2.23 & 3.55 \\
\hline Negative net wealth & -0.42 & 0.22 & 0.43 & 0.66 & 1.00 \\
\hline Locus of control: own ${ }^{\wedge \wedge}$ & 0.67 & 0.36 & 0.96 & 1.95 & 3.98 \\
\hline \multicolumn{6}{|l|}{ Bad risk factors } \\
\hline Constant & $-1.87^{* *}$ & 0.55 & & & \\
\hline Age 30-44 & -0.41 & 0.31 & 0.36 & 0.67 & 1.23 \\
\hline Have thought about SLS & 0.58 & 0.30 & 1.00 & 1.78 & 3.19 \\
\hline Low financial knowledge & $-0.68^{*}$ & 0.33 & 0.27 & 0.51 & 0.97 \\
\hline Female & -0.44 & 0.27 & 0.38 & 0.64 & 1.09 \\
\hline NZ Euro sole ethnicity & $0.70^{*}$ & 0.31 & 1.11 & 2.02 & 3.67 \\
\hline High financial knowledge & -0.39 & 0.34 & 0.35 & 0.68 & 1.32 \\
\hline Negative net wealth & 0.06 & 0.29 & 0.60 & 1.06 & 1.89 \\
\hline Locus of control: own ${ }^{\wedge \wedge}$ & 0.63 & 0.53 & 0.67 & 1.87 & 5.26 \\
\hline \multicolumn{6}{|l|}{ Good risk factors } \\
\hline Constant & $-1.25^{* *}$ & 0.45 & & & \\
\hline Age 30-44 & -0.45 & 0.24 & 0.39 & 0.63 & 1.02 \\
\hline Have thought about SLS & $0.67^{* *}$ & 0.24 & 1.22 & 1.95 & 3.13 \\
\hline Low financial knowledge & $-0.59^{*}$ & 0.28 & 0.32 & 0.55 & 0.95 \\
\hline Female & 0.17 & 0.22 & 0.77 & 1.18 & 1.81 \\
\hline NZ Euro sole ethnicity & -0.32 & 0.23 & 0.47 & 0.73 & 1.14 \\
\hline High financial knowledge & 0.13 & 0.28 & 0.66 & 1.14 & 1.96 \\
\hline Negative net wealth & -0.48 & 0.25 & 0.38 & 0.62 & 1.01 \\
\hline Locus of control: own ${ }^{\wedge \wedge}$ & $1.95^{* *}$ & 0.69 & 1.81 & 7.06 & 27.50 \\
\hline
\end{tabular}


Table 8.4: Predictors of main reason for choice of SLS: multinomial logistic regression, continued

\begin{tabular}{|c|c|c|c|c|c|}
\hline \multirow{2}{*}{$\begin{array}{l}\text { Each reason relative to } \\
\text { reference category "Other } \\
\text { or don't know" }\end{array}$} & \multicolumn{2}{|c|}{ Coefficient } & \multicolumn{3}{|c|}{ 95\% CI for Odds Ratio } \\
\hline & b & SE & Lower & $\begin{array}{l}\text { Odds } \\
\text { Ratio }\end{array}$ & Upper \\
\hline 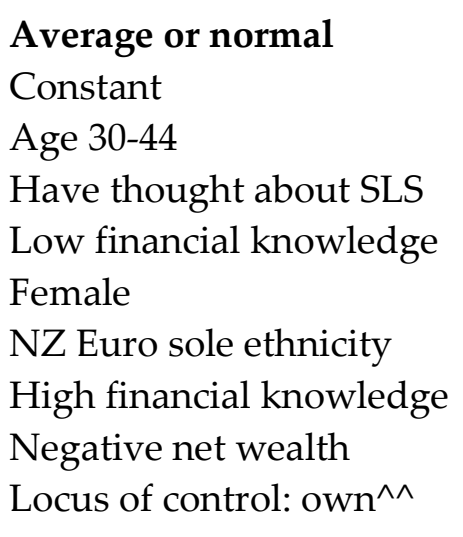 & $\begin{array}{c}-2.89^{* * *} \\
0.53^{*} \\
-0.28 \\
-1.24^{* *} \\
-0.27 \\
-0.14 \\
0.00 \\
-1.36^{* * *} \\
1.13\end{array}$ & $\begin{array}{l}0.60 \\
0.27 \\
0.27 \\
0.36 \\
0.26 \\
0.27 \\
0.31 \\
0.39 \\
0.72\end{array}$ & $\begin{array}{l}1.00 \\
0.45 \\
0.14 \\
0.46 \\
0.51 \\
0.55 \\
0.12 \\
0.75\end{array}$ & $\begin{array}{l}1.70 \\
0.76 \\
0.29 \\
0.76 \\
0.87 \\
1.00 \\
0.26 \\
3.10\end{array}$ & $\begin{array}{c}2.88 \\
1.28 \\
0.59 \\
1.27 \\
1.48 \\
1.83 \\
0.55 \\
12.82\end{array}$ \\
\hline $\begin{array}{l}\text { Notes: } \mathrm{R}^{2}=.21 \text { (Cox and Sn } \\
\text { Significance level: }{ }^{*} \mathrm{p}<.05 \text {; } \\
\text { Predictor variables all have } \\
\text { Sole ethnicity categories co } \\
\text { identified (NZ European, } \\
\text { ^ Strongly or somewhat a } \\
\text { actions". }\end{array}$ & $\begin{array}{l}\text { l), .22 (Na } \\
{ }^{*} \mathrm{p}<.01 ; \\
\text { two categ } \\
\text { apare witl } \\
\text { āori, Asia } \\
\text { ree with ". }\end{array}$ & $\begin{array}{l}\text { ries: s } \\
\text { other } \\
\text {, Paci } \\
\text { My life }\end{array}$ & $\begin{array}{l}\text { ted categ } \\
\text { ole ethni } \\
\text { c) and all } \\
\text { s determ }\end{array}$ & $\begin{array}{l}\text { ry and a } \\
\text { ies sepa } \\
\text { hixed eth } \\
\text { ed by } m\end{array}$ & $\begin{array}{l}94.86^{* * *} \\
\text { other. } \\
\text { ately } \\
\text { icities. } \\
\text { own }\end{array}$ \\
\hline $\begin{array}{l}\text { Forward stepwise method } \\
\text { associations in Pearson's ch }\end{array}$ & d with & riol & idontif & ysir & $\begin{array}{l}\text { cant } \\
\text { tests. }\end{array}$ \\
\hline
\end{tabular}

Those who have given their life expectancy some or a lot of thought also have greater odds for personal good risk factors being the main reason for choosing SLS. A further predictor for having greater odds for good risk factors relative to "other or don't know" is agreeing that life is determined by one's own actions. Those with greater odds of bad risk factors being the main 
reason for choice of SLS are New Zealand Europeans, but no other significant associations were found.

Few significant associations were found to predict each category of main reason for choosing SLS. There are likely to be other predictors unavailable in these data. However, a pattern emerges that the main reason given for SLS is based primarily on thoughtfulness about lifespan, view on locus of control and level of financial knowledge. Demographic and socio-economic factors are conspicuously absent, except that those of New Zealand European sole ethnicity are more likely to give bad risk factors or genes as main reason than say "other or don't know". This could be because of a cultural tendency to give a definite answer rather than genes or bad risk factors being more prevalent for this group. Finally, given that people are more likely to start to consider lifespan after the age of 30 - as shown in Figure 8.4 - it is perhaps not surprising that those aged just above 30 do not have a clear reason for their choice of SLS and so have greater odds of giving the response "average or normal".

\section{Summary: importance of the reason for choosing SLS}

The five main reasons for choice of SLS in the New Zealand adult population are illustrated in Figure 8.6. These reasons are significant as they start to get behind the reason why people choose the SLS they do. Because five different main reasons summarise a larger number of different reasons; and because there are different profiles of those most likely to give each reason, efforts by previous writers to model population choice of SLS as if it were homogenous appear flawed. 
Figure 8.6: Main reason for choosing SLS, grouped, and predictors of being in each group

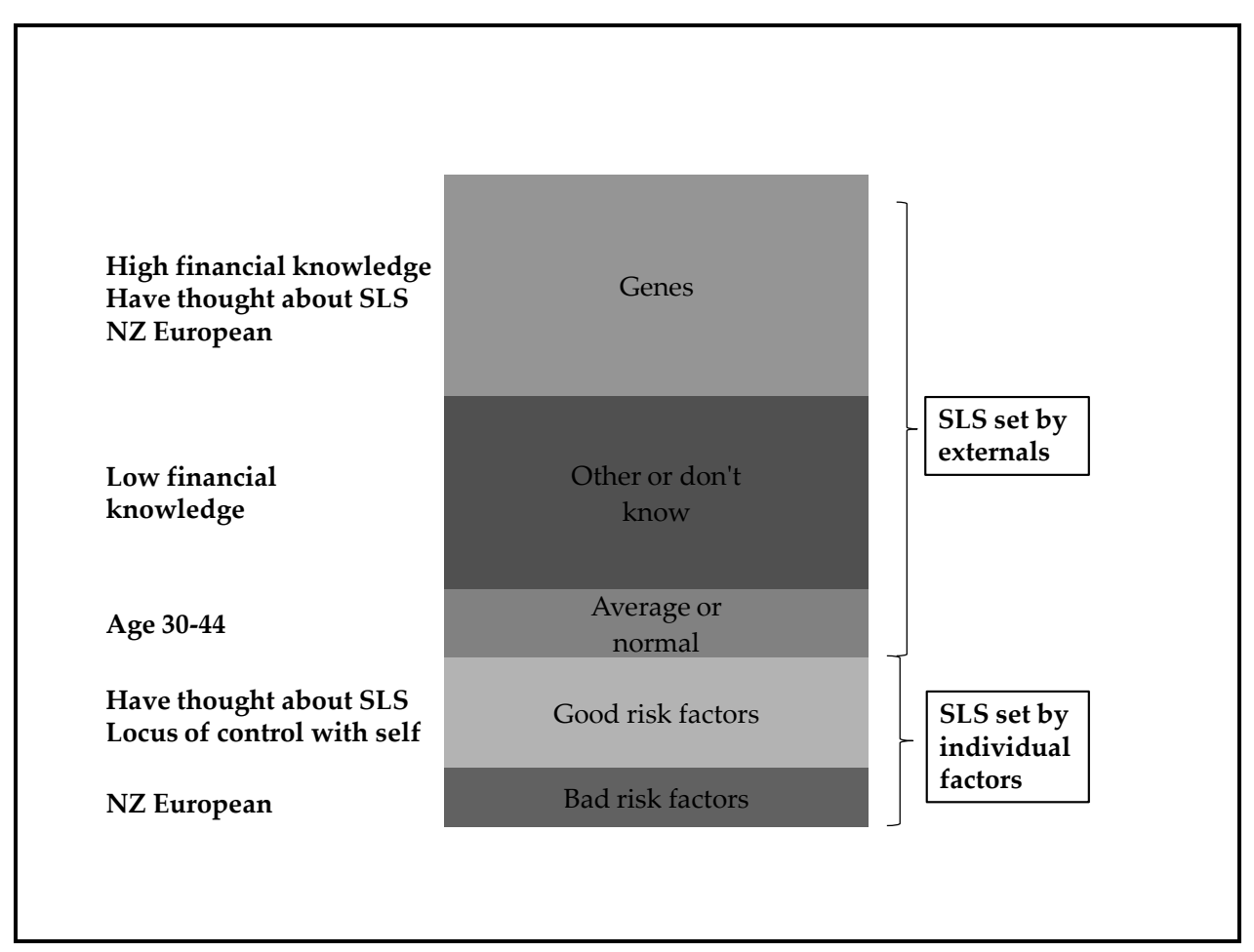

Source: Analysis of Financial Knowledge 2009 survey responses.

Height represents estimated percentage of population in each category, 100 per cent $=850$.

International literature has used three models for how people think about and choose their SLS, as defined and discussed in section 7.2. In the 'normalising' model, adults choose their SLS according what they think is normal in a reference group of like people. In the 'planning for death' model, people choose their SLS based on personal anxiety about death or experiences of death among family. In the 'life planning' model, SLS is used as an assumption in order to plan for retirement.

The results of this survey suggest that all three models may apply within groups of the New Zealand population, but the models do not seem a complete explanation for why people choose the SLS they do, or how they 
think about lifespan. The importance of financial knowledge level as a predictor for main reason for SLS choice seems to indicate that thinking about SLS is linked to financial planning, probably linked to retirement. Further evidence of this is considered in the next chapter. The planning for death model seems more relevant to the large group giving genes or family history as the main reason for SLS. The normalising model clearly applies to those giving "average/normal" as the main reason, but it could also apply to the "other or don't know" group, and to those taking personal risk factors into account, with a different norm. The number and variety of reasons given in this survey suggest a diversity of ways of thinking about lifespan within the population that summary models cannot describe adequately.

For any one individual, the relative importance of genes or risk factors for actual longevity is impossible to estimate unless facts are known on, for example, a smoking-related illness or a hereditary disease. However, biodemographers have proposed that one-quarter of the variation in adult lifespans could be explained by genetic factors, one quarter by early life conditions and half by conditions in later life (Vaupel et al. 1998). 'Later life conditions' includes the health issues and behavioural factors that would be part here of good or bad risk factors. One-third of the sample gave genes as the main reason for SLS choice, and one-quarter personal risk factors. Compared to the Vaupel hypothesis, New Zealanders appear to weight genetic factors more and risk factors less.

Further, almost three-quarters of the population state a first explanation for their choice of SLS that is external to them, either genetics, being average or normal, some other explanation, or none. This suggests that health promotion 
messages - insofar as the connection from healthier personal behaviour to improving lifespan is concerned - may fall on unreceptive ears much of the time.

Just over one fifth of the New Zealand adult population (aged 15+ years) smokes on a regular basis (Ministry of Health Tobacco Use Survey 2008). Smoking is thought to be a well-understood health risk factor. Yet fewer than 10 per cent (9.1 per cent, weighted estimate, sample error $+/-0.6$ per cent) admit that their bad risk factors may affect SLS, and of the whole sample only 3.5 per cent specifically said they smoked. Previous surveys have linked smoking behaviour with SLS, and asserted that smokers generally underestimate the risk to their longevity. This survey suggests that most smokers do not first think of their smoking when they choose their SLS. Further, if behavioural risk factors are not being considered by the majority of the population in setting their SLS, it is not surprising that international studies have not found conclusive or consistent evidence for the 'accuracy' of individuals' SLS compared to the effect of such factors.

Finally, in terms of public messages around improving longevity for superannuation and retirement planning, as so many people believe in an external or given reason for lifespan, it may be that they are receptive to evidence that it is improving. The counter to that may be that as a sizeable group have taken evidence from their family history or their own personal risk factors, the power of individual anecdote or belief in personal risk may override the general message. It could be that only the tenth of the adult population who say that they chose their SLS because it is 'average or normal' are receptive to public information on average population lifespans. 


\subsection{Lifespan underestimation}

The pattern of diverse reasons given for choosing SLS suggests that choice is not evidence-based for many New Zealanders. This section explores how the SLS chosen fit the lifespan evidence available. Whether the SLS show over- or underestimation of lifespan can only be seen by comparing the SLS responses against each other - which tests whether those at relative risk of low lifespan have relatively lower SLS - or by comparing with relevant measures of likely lifespan for the population from which the sample is drawn. In what follows, first, factors predicting high or low SLS are investigated and responses examined for directional accuracy. Next, the gaps between SLS and life table lifespans are calculated and the profile of those most likely to over- or underestimate SLS described.

\subsubsection{Predicting high or low SLS}

The profile of those choosing relatively high or low SLS should give some insight into whether the choice is directionally 'accurate' relative to the actuarial risk inherent in the profile, or related to subjective rather than known risk factors, or simply random. In particular, those likely to choose extreme high or low SLS are likely to be over- or underestimating respectively compared to life tables, so are a potential source of the greatest inaccuracy.

Binary logistic regression was used to identify predictive characteristics of those more or less likely to choose high or low SLS and to choose extreme values. The "don't knows" were eliminated from the sample to construct three dichotomous dependent variables for those who gave an SLS response:

- Very low SLS of 65 or below compared to SLS 70 or above (n=65/661). 
- Above median SLS of 85 or above compared to SLS 80 or below $(n=355 / 372)$.

- Very high SLS of 100 or above compared to SLS 95 or below (n=61/665).

This grouping of the sample in these groups is illustrated in Figure 8.7, and the results of the regression are in Table 8.5.

Figure 8.7: SLS, above and below median and extreme groups, estimated percentage of population

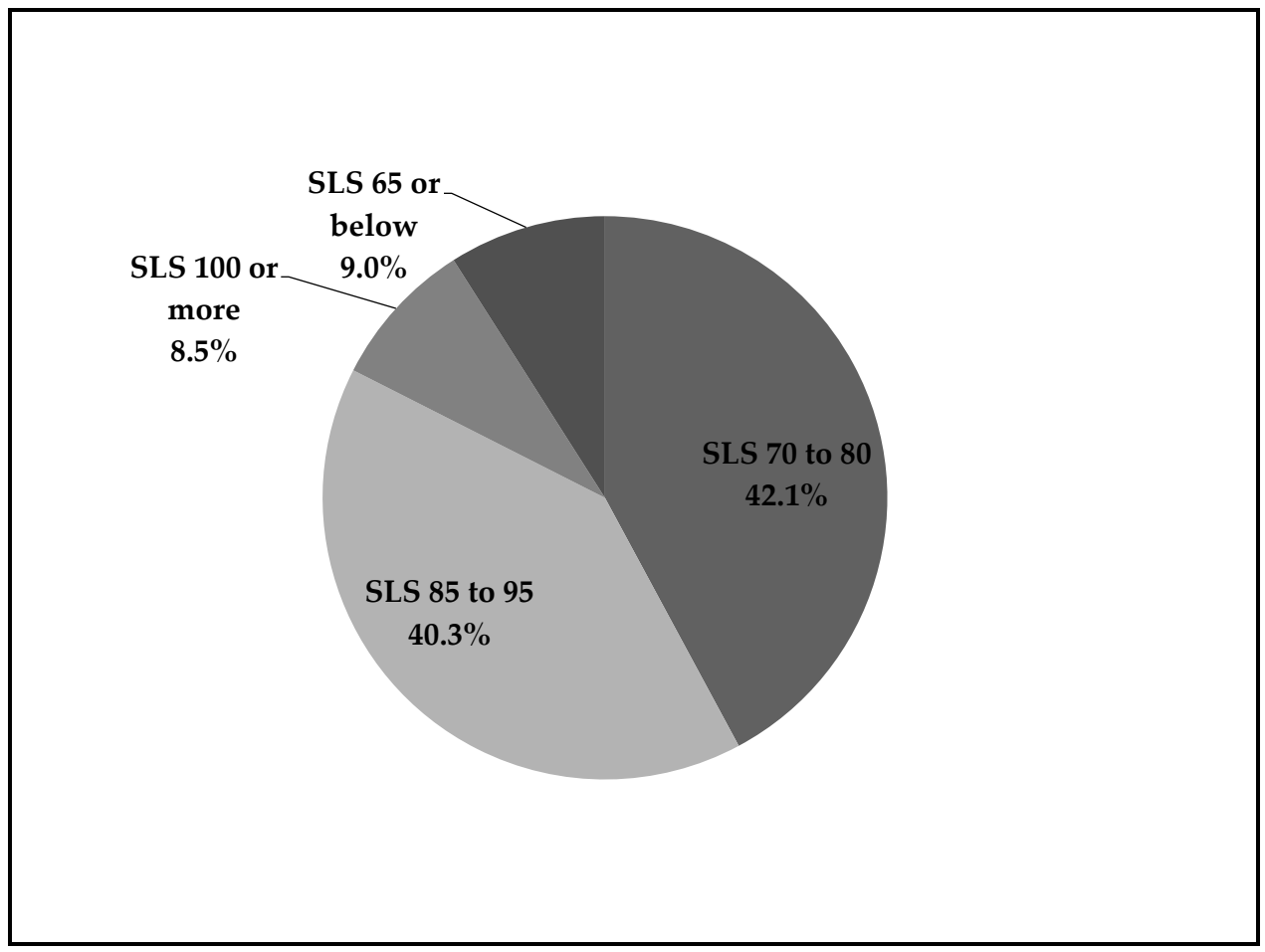

Source: Analysis of Financial Knowledge 2009 survey responses. $\mathrm{n}=726$ who gave an SLS. 
Table 8.5: Predictors of SLS: binary logistic regressions

\begin{tabular}{|c|c|c|c|c|c|}
\hline & & & \multicolumn{3}{|c|}{ 95\% CI for Odds Ratio } \\
\hline & \multicolumn{2}{|c|}{ Coefficient } & \multirow[b]{2}{*}{ Lower } & \multirow{2}{*}{$\begin{array}{l}\text { Odds } \\
\text { Ratio }\end{array}$} & \multirow[b]{2}{*}{ Upper } \\
\hline & b & SE & & & \\
\hline $\begin{array}{l}\text { SLS } 65 \text { or below as } \\
\text { compared to } 70 \text { or more }\end{array}$ & & & & & \\
\hline Constant & $-4.23^{* * *}$ & 0.40 & & & \\
\hline First reason other/DK & $1.10^{* *}$ & 0.40 & 1.39 & 3.01 & 6.54 \\
\hline First reason 'average' & $1.37^{* *}$ & 0.49 & 1.52 & 3.93 & 10.17 \\
\hline Bad risk factors & $2.85^{* * *}$ & 0.42 & 7.63 & 17.22 & 38.91 \\
\hline Age 60 and above & $-2.60^{*}$ & 1.07 & 0.01 & 0.07 & 0.60 \\
\hline Age below 30 & 0.61 & 0.33 & 0.96 & 1.84 & 3.51 \\
\hline Low financial knowledge & $1.04^{* *}$ & 0.33 & 1.49 & 2.82 & 5.35 \\
\hline Locus of control: others ${ }^{\wedge}$ & $0.69^{*}$ & 0.32 & 1.06 & 2.00 & 3.76 \\
\hline Negative net wealth & $0.71^{*}$ & 0.32 & 1.09 & 2.04 & 3.81 \\
\hline $\begin{array}{l}\text { SLS } 85 \text { or above as } \\
\text { compared to } 80 \text { or below }\end{array}$ & & & & & \\
\hline Constant & -0.70 & 0.45 & & & \\
\hline Māori sole ethnicity & $-0.76^{*}$ & 0.32 & 0.25 & 0.47 & 0.87 \\
\hline Asian sole ethnicity & $-0.72^{*}$ & 0.29 & 0.28 & 0.49 & 0.87 \\
\hline Genes as first reason & $0.46^{*}$ & 0.19 & 1.10 & 1.58 & 2.28 \\
\hline Good risk factors & $0.99^{* * *}$ & 0.23 & 1.72 & 2.69 & 4.23 \\
\hline Bad risk factors & $-1.57^{* * *}$ & 0.38 & 0.10 & 0.21 & 0.44 \\
\hline Female & $0.34^{*}$ & 0.16 & 1.02 & 1.40 & 1.92 \\
\hline Age 60 and above & $0.63^{* *}$ & 0.21 & 1.25 & 1.87 & 2.80 \\
\hline Locus of control: own ${ }^{\wedge \wedge}$ & $-1.23^{* *}$ & 0.43 & 0.13 & 0.29 & 0.68 \\
\hline $\begin{array}{l}\text { SLS } 100 \text { or above as } \\
\text { compared to } 95 \text { or below }\end{array}$ & & & & & \\
\hline Constant & $-5.30^{* * *}$ & 0.98 & & & \\
\hline Have thought about SLS & $1.07^{* *}$ & 0.39 & 1.35 & 2.93 & 6.33 \\
\hline Genes as first reason & $1.97^{*}$ & 0.93 & 1.14 & 7.14 & 44.51 \\
\hline First reason other/DK & $2.41^{*}$ & 0.94 & 1.78 & 11.17 & 70.17 \\
\hline Good risk factors & $3.16^{* *}$ & 0.92 & 3.87 & 23.60 & 144.00 \\
\hline Low financial knowledge & $1.15^{* * *}$ & 0.31 & 1.73 & 3.16 & 5.78 \\
\hline Negative net wealth & $-0.773^{*}$ & 0.36 & 0.23 & 0.46 & 0.94 \\
\hline Have will & $-0.99 * *$ & 0.32 & 0.20 & 0.37 & 0.70 \\
\hline
\end{tabular}


Source for Table 8.5:

Analysis of Financial Knowledge 2009 survey responses.

Notes:

SLS at 65/70:

$\mathrm{R}^{2}=.14$ (Cox and Snell), .31 (Nagelkerke). Model $\chi^{2}(8)=109.54^{* * *}$

SLS at 80/85:

$\mathrm{R}^{2}=.13$ (Cox and Snell), .18 (Nagelkerke). Model $\chi^{2}(8)=102.42^{* * *}$

SLS at 95/100:

$\mathrm{R}^{2}=.10$ (Cox and Snell), .22 (Nagelkerke). Model $\chi^{2}(7)=73.77^{* * *}$

Significance level: ${ }^{*} \mathrm{p}<.05 ;{ }^{* *} \mathrm{p}<.01 ;{ }^{* * *} \mathrm{p}<.001$

Predictor variables all have two categories: stated category and all other.

Sole ethnicity categories compare with other sole ethnicities separately identified (NZ European, Māori, Asian, Pacific) and all mixed ethnicities.

$\wedge$ Strongly or somewhat agree with "My life is controlled by the actions of other people".

$\wedge \wedge$ Strongly or somewhat agree with "My life is determined by my own actions".

All variables were tested for each of the three models.

Variables excluded by stepwise regression from all models:

New Zealand European sole ethnicity

Age 30-44

Age 45-59

Tertiary or postgraduate education

High personal income; over $\$ 30,000$ pa

High net wealth; over $\$ 301,000$

Have thought about financial planning for retirement

Strongly or somewhat agree with "My life is determined by things beyond my control".

In Medium financial knowledge group

In High financial knowledge group

Forward stepwise models used; backwards models tested but did not improve fit. 
The regression models do not explain all of the variance that exists, as the $\mathrm{R}^{2}$ indicators are low. However, the picture that emerges suggests that the reason for SLS choice is as important as the few actuarial factors that seem to be relevant. Further, both extremes of the SLS distribution are also associated with having a low level of financial knowledge.

\section{Very low SLS: Pessimism and lack of knowledge}

Not surprisingly, those aged 60 and over are significantly less likely to choose an SLS of 65 or below than those aged under 60 , but there appears to be no significant difference within ages under 60 in propensity to choose a very low SLS. People with negative net wealth are more likely to choose a very low SLS than those with positive net wealth. These significant predictor variables are the only ones linked to actuarial risk factors. It therefore appears that something other than an appraisal of population mortality risk influences the choice of a very low SLS.

The high significance of bad risk factors as the first reason for choosing SLS, with odds ratio of 17 , suggests that people reasoning on the basis of their individual mortality risk factors from behaviour such as smoking, poor diet or lack of exercise or a health problem, are likely to choose a very low SLS. However, as discussed earlier, the proportion of people reasoning SLS on the basis of bad risk factors appears low compared to the prevalence of such factors in the population. Those taking such risks into account when choosing their SLS are not necessarily doing so correctly, but are more pessimistic about their health or mortality chances than those with the same bad risks but who do not reason their choice of SLS on the same basis. 
This suggests that those choosing a very low SLS are influenced, perhaps unduly, by pessimism about life chances. This is confirmed as those who agree that their life is controlled by the actions of other people are twice as likely to choose a very low SLS compared to others. The pessimism also seems linked to lack of knowledge about lifespans and planning. Specifically, those who say "don't know" or similar when asked why they chose their SLS are more likely to choose a very low SLS than others. Those who offer the reason that they are "average" or "normal" are also more likely to do so. And low financial knowledge is almost as salient a predictor with an odds ratio of nearly three.

\section{Above median SLS: Optimism strengthened by age and gender}

The odds of those aged 60 and over choosing an SLS or 85 or more are nearly twice those of younger respondents. The odds for females are 1.4 times higher than that of males. Those giving Māori as sole ethnicity are less likely to choose an above median SLS. These properties are in the correct direction compared to mortality risk (although not necessarily of the correct size, which is not tested here). However, the remaining predictor variable associated with actuarial risk appears to be in an incorrect direction: those giving Asian as sole ethnicity are less likely to choose an above median SLS than others, but population risk would predict longer lifespans on average for those of Asian ethnicity. However, given the small subsample of Asian ethnicity this result should be considered with caution.

The other variables cannot be explained actuarially as they are related to how people think about SLS and life in general. Those making genes the first reason for their SLS are more likely to choose an above median SLS than other 
respondents. Those reasoning on the basis of good risk factors are more likely to give an above median SLS, whereas those reasoning on the basis of their bad risk factors are significantly less likely to do so. Those who agree that life is determined by their own actions are also less likely to choose a high SLS. Taken together, these results imply a degree of optimism that either externalities or individual good health behaviour will influence longevity positively.

\section{Very high SLS: Optimism with doubtful rationale}

The lack of any variable of actuarial significance in the regression model derived for the highest SLS of 100 or more is striking. Instead, optimism appears to be the strongest influence. Those who have given the age they might live to some thought are more likely to choose a very high SLS or more compared to others. However, having thought about SLS is not guarantee of choosing an accurate SLS, or being able to use that SLS in planning effectively. Those in the low financial knowledge group are also more likely to choose a very high SLS than respondents with greater financial knowledge scores.

Again, the reasoning behind choice of SLS was highly significant for predicting a very high SLS, with an odds ratio of 24 for those reasoning on the basis of good risk factors and an odds ratio of seven for those reasoning on the basis of genes. However, those who said "don't know" when asked why they chose the SLS they did also were more likely to choose a very high SLS. This is consistent with a general theme of optimism without good rationale for those hoping for a lifespan of 100 or more, notwithstanding that some respondents will have some grounds for a longer than average lifespan on the basis of family history or very healthy lifestyle. 
Those who have negative net wealth are less likely to choose a very high SLS, the converse of being more likely to choose a very low SLS. Those who have made a will are also less likely to choose the highest SLS. Perhaps for this latter group the necessity to confront mortality in a practical way has led to a more conservative assessment than those who claim to have thought about how long they will live, but have thought about it in the abstract.

\section{Summary: Reasoning behind SLS is a key predictor}

Taken together, the results from these three models suggest that the usual actuarial predictors of age and gender are not the only things people think of when choosing an SLS. Indeed, for those who give the highest or lowest value of SLS, actuarial parameters are hardly important at all. Further, other factors that are known to make a different to actual mortality risk such as ethnicity and other socio-economic indicators seem to be relatively absent in New Zealanders' consideration of SLS. The exceptions are that Māori sole ethnicity seems to be an indicator for below rather than above median SLS and that negative net wealth is associated with the lowest SLS.

The stronger predictor of high or low SLS appears to be the reasoning behind choosing SLS. Individual good or bad risk factors are highly significant predictors of above or below median SLS and especially for very high or very low SLS. However, the associations found with general locus of control, with low financial knowledge and with having no particular reasoning for choice of SLS suggest that for many, SLS is not an informed or thoughtful prediction. 
No previous study of subjective longevity expectations explored the reasons why people gave the SLS or subjective probability of survival (SPS) they did, although some small studies found an association of family longevity history with SLS (Brouwer and van Exel 2005; Hamermesh 1985). The new finding here is that family longevity history may be an important explanation for many people, and individual risk factors may be for others, but people reason differently - if they do so at all - and this reasoning has significant influence on choice of SLS. Further, rational understanding of mortality risk seems to play little part in setting SLS. This finding casts doubt on previous interpretations of surveys that imputed accuracy in given SLS or SPS to respondents' understanding of their own risk factors as if all respondents were thinking in that way.

\subsubsection{Over- and underestimation of SLS}

In the earlier part of this chapter, it was shown that the aggregate SLS of New Zealanders did not obviously underestimate relative to age-independent population lifespan indicators which ignored future mortality improvement. However, the distribution of SLS showed a wide range of SLS responses, and variation by gender. Here, the distributions of the gaps between SLS and age-, gender- and time-dependent life table measures are evaluated to explore what factors contribute to congruence with life tables or over- or underestimation of lifespan.

Four life tables for the New Zealand population are used, as provided by Statistics New Zealand: the latest complete period life table as at the time of the survey and the cohort life table for all ages in calendar year 2009. The period table takes no account of potential future change in age-specific 
mortality risk. The cohort table does, and has three versions, one for each of the main scenarios for future mortality in the 2009-base population projections. The gap between SLS and rounded life table measures was calculated for each respondent by age and gender. A positive gap represents underestimation of lifespan relative to the comparator life table; a negative gap overestimation. Table 8.6 summarises key measures of the four distributions of the gaps between SLS and each of the life tables.

The period life table itself is an underestimation of likely lifespan, as it does not take future mortality improvement into account. The cohort life table does so. This explains the pattern of increasing underestimation moving down through the different life tables of Table 8.6. Relative to the period life table, male SLS appear to be congruent on average, although the gap ranges from overestimating by 25 years to underestimating by 20 years. Women underestimate on average by 2.8 years to the period life table, with a maximum underestimation of 25 years. For each life table comparator, the SLS for women are a greater underestimate than the SLS for men.

Figure 8.8 shows how the prevalence of underestimation of SLS increases through the progression of life tables as the greater force of assumed future mortality improvement lengthens estimated lifespans for each age and gender. 
Table 8.6: Summary of gap between life table estimated lifespan

(by age and gender) and SLS for men and women, in years

\begin{tabular}{|c|c|c|}
\hline & \multicolumn{2}{|c|}{ Gap, in years } \\
\hline & $\begin{array}{c}\text { Men } \\
n=365\end{array}$ & $\begin{array}{c}\text { Women } \\
n=361\end{array}$ \\
\hline Period life table & & \\
\hline Mean & 0.0 & 2.8 \\
\hline Median & 0.0 & 0.0 \\
\hline Mode & 0 & 0 \\
\hline Minimum & -25 & -20 \\
\hline Maximum & 20 & 25 \\
\hline Cohort life table, high mortality & & \\
\hline Mean & 1.2 & 2.8 \\
\hline Median & 0.0 & 0.0 \\
\hline Mode & 0 & 0 \\
\hline Minimum & -25 & -20 \\
\hline Maximum & 25 & 25 \\
\hline Cohort life table, medium mortality & & \\
\hline Mean & 4.2 & 4.5 \\
\hline Median & 5.0 & 5.0 \\
\hline Mode & 0 & 0 \\
\hline Minimum & -20 & -20 \\
\hline Maximum & 25 & 30 \\
\hline Cohort life table, low mortality & & \\
\hline Mean & 5.5 & 7.3 \\
\hline Median & 5.0 & 5.0 \\
\hline Mode & 0 & 5 \\
\hline Minimum & -20 & -15 \\
\hline Maximum & 30 & 30 \\
\hline
\end{tabular}

Source: Analysis of Financial Knowledge 2009 survey responses.

Note: A positive gap represents underestimation of lifespan relative to the comparator life table; a negative number overestimation. If the oldest respondent were aged 99 instead of 89 as assumed then the mean gap to the period life table would increase by 0.2 years for women. The cohort table provided stops at age 90 , so a similar sensitivity test could not be performed. The cohort table of life expectancy by age in calendar year 2009 consistent with 2009-base projections was provided by Statistics New Zealand (April 2010). 
Figure 8.8: Estimated percentage of the population with SLS higher (overestimate), congruent or lower (underestimate) relative to population life tables

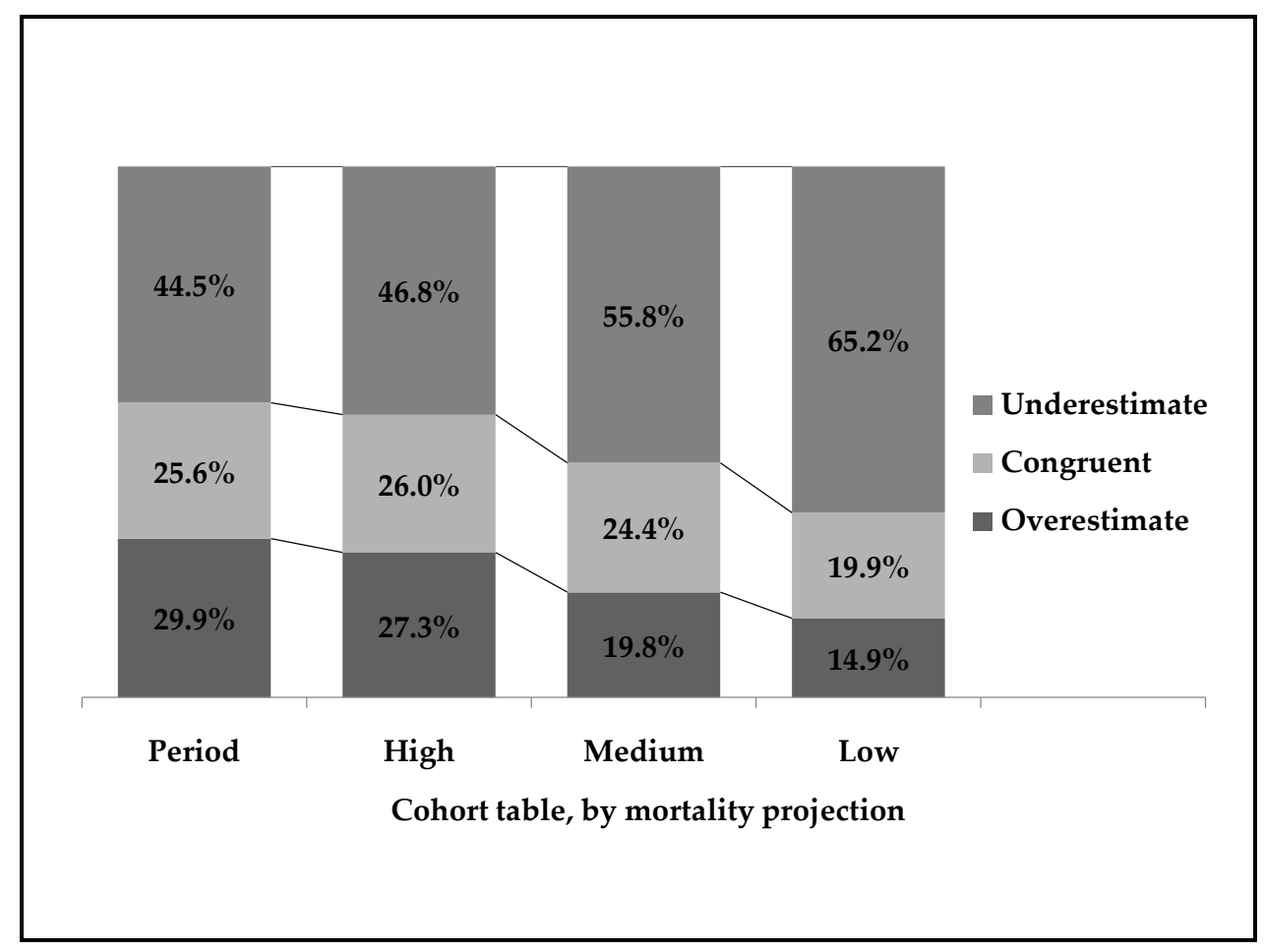

Source: Analysis of Financial Knowledge 2009 survey responses. $\mathrm{n}=726$ who gave an SLS. Congruence implies the SLS is within the range of relevant life table values for the age group within which the individual lies.

Figure 8.9 illustrates the distribution of the gap relative to the period table by gender. Figure 8.10 shows the distribution of the gap relative to the Low Mortality cohort table, illustrating how the prevalence of underestimation increases as the assumption for future mortality improvement becomes stronger. 
Figure 8.9: Gap between SLS and period life table, estimated percentage of the population by gender

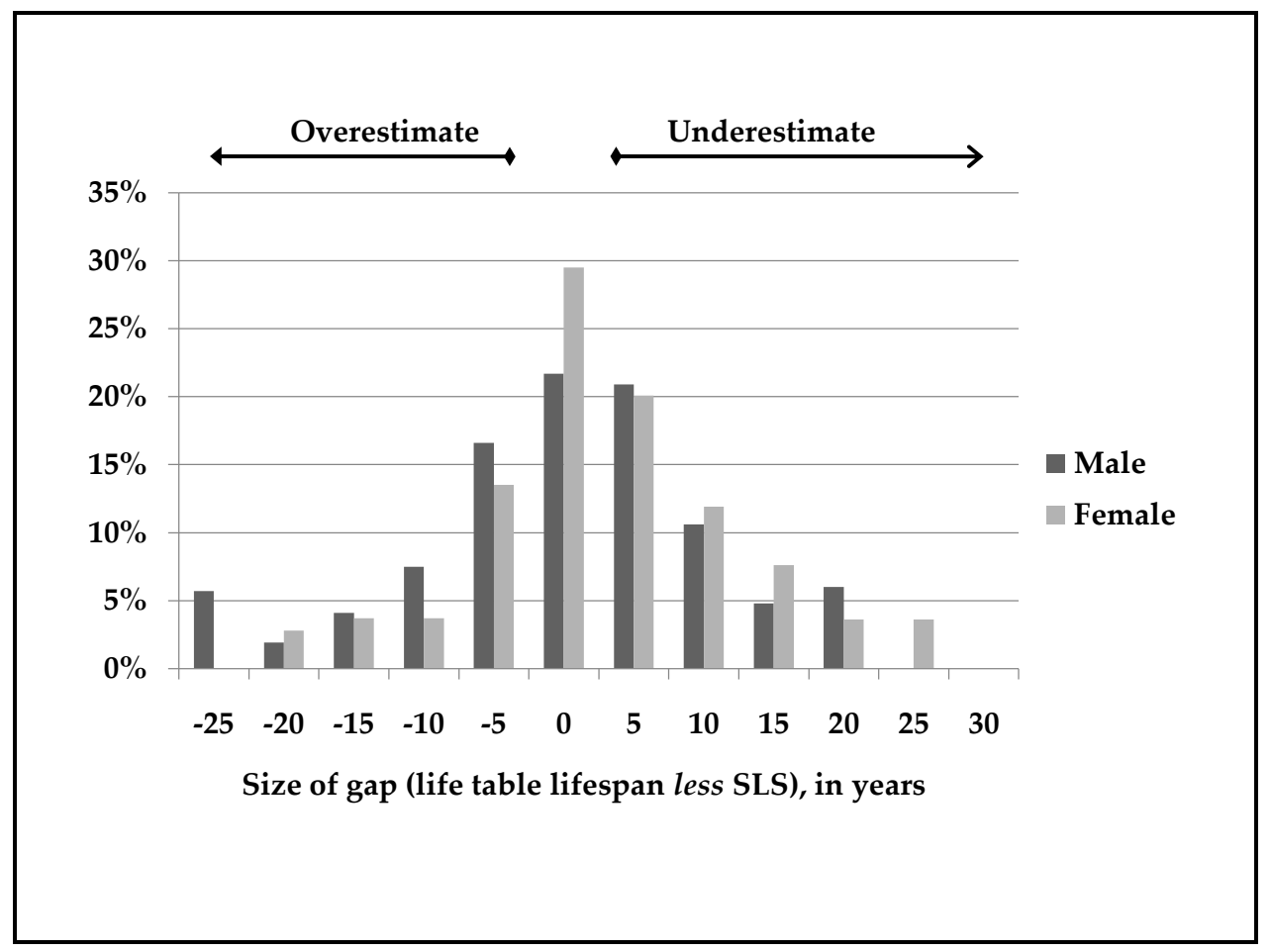

Source: Analysis of Financial Knowledge 2009 survey responses. $\mathrm{n}=365$ male, 361 female who gave an SLS.

Chapter 5 suggested that the most plausible mortality outcome for New Zealand could be the Low Mortality cohort table. Compared to this benchmark, the best estimate of underestimation of SLS on average is 5.5 years for men and 7.3 years for women. O'Brien et al (2005) found underestimation relative to a British cohort table with mortality assumptions in a range similar to the medium to low mortality assumptions of the New Zealand table of 4.6 years for males and 6.0 years for females. Thus this set of results is highly consistent with those of the only comparable international survey of adult population SLS. 
Figure 8.10: Gap between SLS and Low Mortality cohort life table, estimated percentage of the population by gender

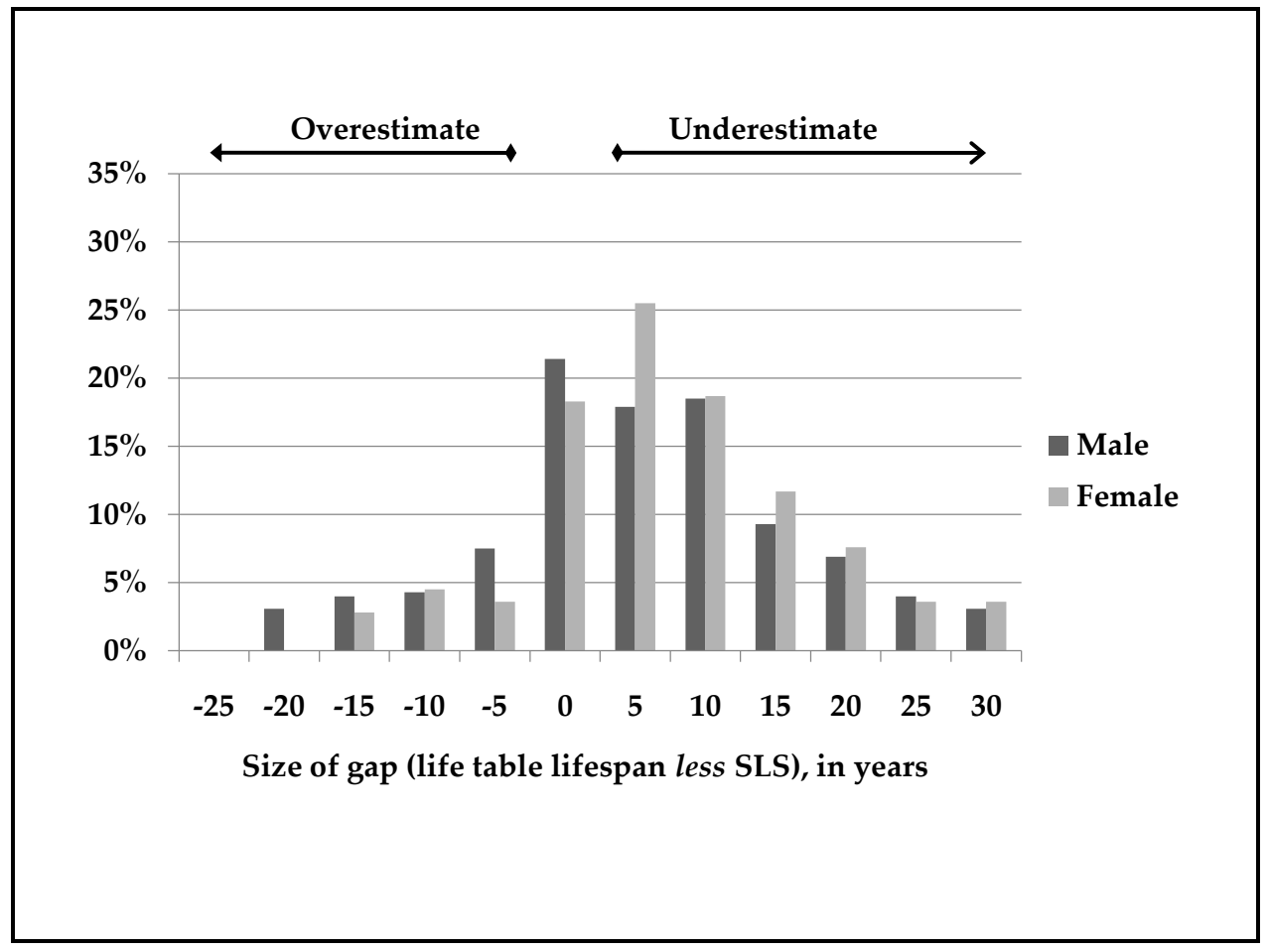

Source: Analysis of Financial Knowledge 2009 survey responses. $\mathrm{n}=365$ male, 361 female who gave an SLS.

In order to identify the characteristics of those most at risk of underestimating lifespans, profiling of the gap distributions were carried out for the gap relative to the period table and the Low Mortality cohort table. Categorical tests of association were carried out with the distribution of SLS to the period gap split into three groups: congruent to the life table (that is, the gap was around zero); overestimates (the left hand side of the gap distribution); and, underestimates (the right hand side of the gap distribution). Predictive variables for being in the over- and underestimating group instead of the congruent group were identified by multinomial regression, following exploratory analysis with Pearson's chi-squared tests (not shown). Results are in Table 8.7. 
Table 8.7: Predictors of over- and underestimation of SLS compared to period life table: multinomial logistic regression

\begin{tabular}{|c|c|c|c|c|c|}
\hline & & & \multicolumn{3}{|c|}{ 95\% CI for Odds Ratio } \\
\hline & \multicolumn{2}{|c|}{ Coefficient } & \multirow[b]{2}{*}{ Lower } & \multirow{2}{*}{$\begin{array}{l}\text { Odds } \\
\text { Ratio }\end{array}$} & \multirow[b]{2}{*}{ Upper } \\
\hline & $\mathbf{b}$ & SE & & & \\
\hline \multicolumn{6}{|l|}{$\begin{array}{l}\text { Overestimate vs. } \\
\text { congruent }\end{array}$} \\
\hline Constant & -0.56 & 0.95 & & & \\
\hline Locus of control: others^ ${ }^{\wedge}$ & $0.64^{* *}$ & 0.24 & 1.18 & 1.89 & 3.02 \\
\hline Locus of control: own ${ }^{\wedge \wedge}$ & 0.36 & 0.45 & 0.59 & 1.44 & 3.49 \\
\hline Age 60 and above & $-1.23^{* * *}$ & 0.28 & 0.17 & 0.29 & 0.50 \\
\hline Bad risk factors & -1.42 & 0.79 & 0.05 & 0.24 & 1.14 \\
\hline Genes as first reason & $0.53^{*}$ & 0.26 & 1.03 & 1.70 & 2.83 \\
\hline NZ Euro sole ethnicity & -0.33 & 0.25 & 0.44 & 0.72 & 1.17 \\
\hline Good risk factors & $1.12^{* * *}$ & 0.31 & 1.66 & 3.06 & 5.63 \\
\hline Male & $0.87^{* * *}$ & 0.22 & 1.56 & 2.39 & 3.67 \\
\hline High net wealth & $-0.58^{*}$ & 0.23 & 0.36 & 0.56 & 0.88 \\
\hline \multicolumn{6}{|l|}{$\begin{array}{l}\text { Underestimate vs. } \\
\text { congruent }\end{array}$} \\
\hline Constant & 1.01 & 0.63 & & & \\
\hline Locus of control: others^ ${ }^{\wedge}$ & $0.63^{* *}$ & 0.22 & 1.21 & 1.87 & 2.90 \\
\hline Locus of control: own ${ }^{\wedge \wedge}$ & $1.45^{* *}$ & 0.48 & 1.68 & 4.26 & 10.85 \\
\hline Age 60 and above & $-0.75^{* *}$ & 0.24 & 0.30 & 0.47 & 0.75 \\
\hline Bad risk factors & $1.31^{* *}$ & 0.40 & 1.69 & 3.72 & 8.15 \\
\hline Genes as first reason & -0.20 & 0.23 & 0.52 & 0.82 & 1.28 \\
\hline NZ Euro sole ethnicity & $-0.74^{* *}$ & 0.23 & 0.30 & 0.48 & 0.75 \\
\hline Good risk factors & -0.17 & 0.30 & 0.47 & 0.84 & 1.52 \\
\hline Male & 0.07 & 0.20 & 0.72 & 1.07 & 1.59 \\
\hline High net wealth & -0.36 & 0.21 & 0.46 & 0.70 & 1.05 \\
\hline \multicolumn{6}{|c|}{$\begin{array}{l}\text { Source: Analysis of Financial Knowledge } 2009 \text { survey responses. } \\
\text { Notes: } \\
\mathrm{R}^{2}=.22 \text { (Cox and Snell), .24 (Nagelkerke). Model } \chi^{2}(18)=176.32^{* * *} \\
\text { Significance level: }{ }^{*} \mathrm{p}<.05 ;{ }^{* *} \mathrm{p}<.01 ;{ }^{* * *} \mathrm{p}<.001 \\
\wedge \text { Strongly or somewhat agree with "My life is controlled by the actions of } \\
\text { other people". } \\
\wedge \wedge \text { Strongly or somewhat agree with "My life is determined by my own } \\
\text { actions". }\end{array}$} \\
\hline
\end{tabular}


Relative to the low mortality cohort table, an estimated 14.9 per cent of the population gave an SLS that overestimated. Therefore only two groups were formed to compare underestimates (65.2 per cent of sample) with congruent or overestimated SLS (34.8 per cent). The results of binary logistic regression are in Table 8.8.

Table 8.8: Predictors of underestimation of SLS compared to Low Mortality cohort life table: binomial logistic regression

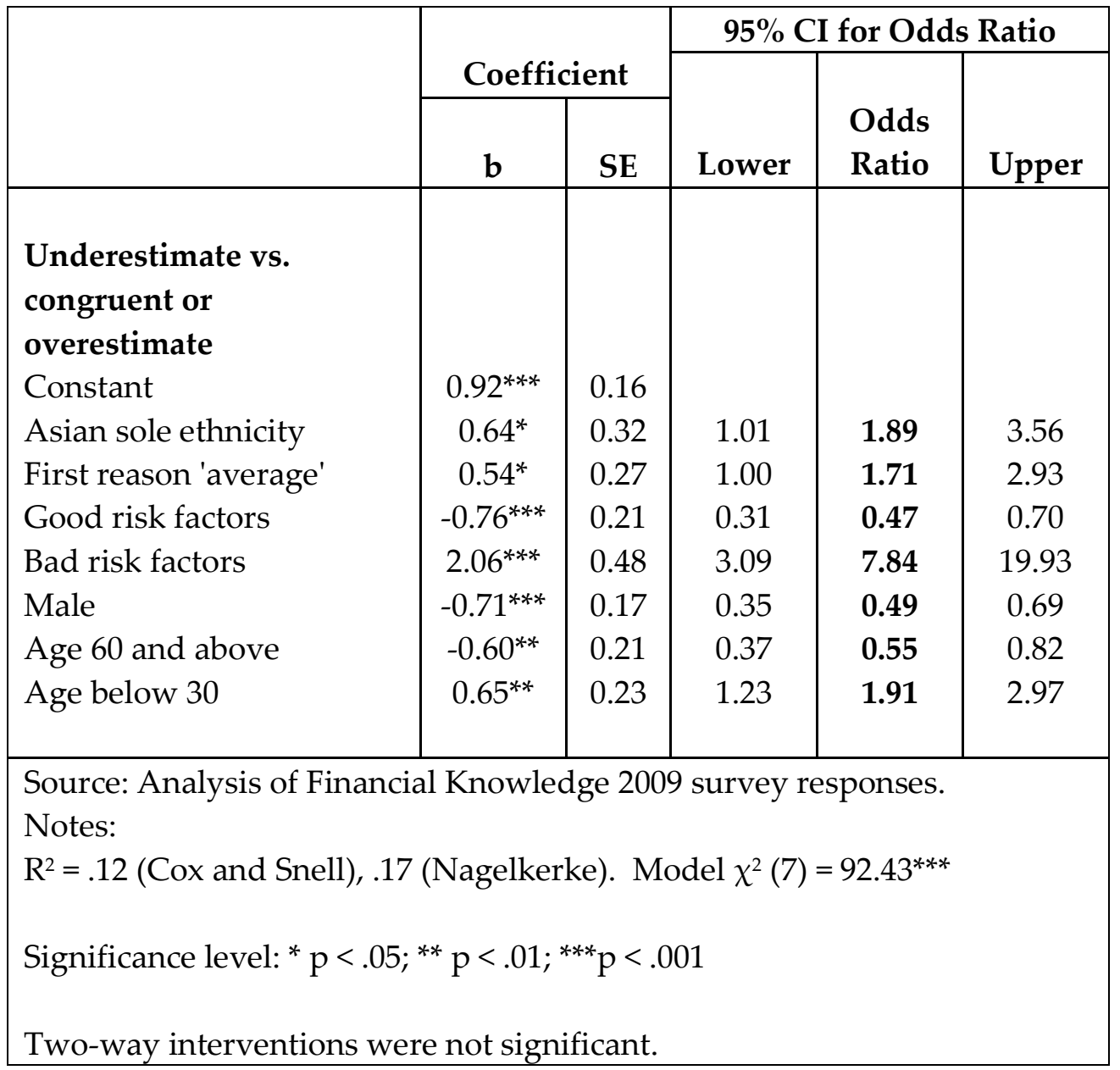




\section{Relative to period life table}

Relative to giving an SLS congruent to the period life table, the odds of overestimating SLS are significantly greater for males, those who give good risk factors as their main reason for choosing SLS, those who give genes as their main reason, or those with net wealth $\$ 300,000$ or below. The odds of underestimating SLS to the period table are greater relative to giving a congruent SLS for those who give bad risk factors as their main reason, those who give ethnicity as something other than solely New Zealand European or those who agree that the life is determined by their own actions. Those aged 60 and over, or who disagree that life is controlled by the actions of others have greater odds for choosing an SLS congruent to the period table, relative to either underestimating or overestimating.

The strength of prediction from personal good or bad mortality risk information is not surprising. This additional information is lost in a population average represented by the life table, although if it were an accurate representation of actual medical risk then would be taken into account by individual underwriting for a life insurance or annuity policy. Whether or not people reasoning their SLS by individual risk factors turn out to be accurate, their SLS is presumably in the right direction relative to the current population table. The greater likelihood of those reasoning on the basis of genes to overestimate suggests a greater tendency to use family history only when it is good, as if longer-living ancestors are more memorable than shorter-living ones when thinking about one's own longevity. Again, people reasoning on the basis of genes may turn out to be accurate in their choice of SLS or not. 
This analysis confirms that, although men choose below median SLS more than women (Table 8.5), men are also more likely to overestimate SLS than women. This is consistent with the aggregate picture of Table 8.6, which showed men on average choosing an SLS congruent to the male period table lifespan and women underestimating relative to the female period table. Assuming that men and women are equally accurate in their prediction of their own longevity, it could be that the male life table underestimates future likely longevity more than the female life table does. Alternatively, if the period tables were an accurate estimation of future longevity for both genders then this picture could be explained by men being relatively less cautious, and more prone to exaggerate SLS than women. This is consistent with the previously made observation that men were less likely to say "don't know" and therefore perhaps more likely to guess than women. A similar explanation could be advanced for why people of lower net worth are more likely to overestimate than choose a congruent SLS.

While it is logical that those who reason on the basis of bad risk factors would have greater odds of underestimating SLS relative to congruence with the period life table, it is less clear why those not of New Zealand European sole ethnicity or who agree that life is controlled by one's own actions would. A possible explanation is that people in these groups tend to think they would have shorter lifespans than what they see as the average, which is represented in some way by the period life table. This is explicable to some extent on the basis of ethnicity, although Asian sole ethnicity would be a counter-example. The locus of control predictor makes sense under this explanation only if people who think that own actions matter believe that their own actions are a mortality risk relative to average. This is not inconsistent with the greater 
odds for people who disagree that the actions of others control their life choosing a congruent SLS, which further suggests that congruence is associated with a norm which is out of individual or others' control.

However, the results are not conclusively consistent with the suggestion that the population models SLS on a norm represented by the period life table. Individual risk factors influence some, but also the greater odds for males to overestimate suggest a fundamental division by gender. Further, people aged 60 and over have greater odds for a congruent SLS to the table relative to either over- or underestimating compared to all ages below 60 . This suggests a further division by age. Therefore, it appears that the population is generally not choosing SLS congruent with the period table across either of the table's two defining characteristics: age or gender.

\section{Relative to Low Mortality cohort life table}

The profile of those more likely to underestimate SLS relative to the Low Mortality cohort table, as shown in Table 8.8, is a more realistic assessment of those actually likely to underestimate their SLS than that relative to the period life table. Underestimating lifespan is more likely for those aged below 30 than older people, although less likely for those aged 60 and over. It is more likely for women than men, all other things equal. It is also more likely for those of Asian sole ethnicity than people of any other ethnicity. Reasoning behind choice of SLS is again important, with those choosing good or bad risks being less or more likely to underestimate respectively and those saying they chose their SLS on the basis of it representing a normal or average lifespan being more likely to, comparing in each case to those giving all other reasons. 
Possible explanations for each of these predictor relationships can be advanced. The youngest ages are more likely to underestimate than other groups because for them the strong mortality improvement assumptions in the low mortality cohort table have longest to take effect. If people are taking a view based on current lifespans they see around them without taking future mortality improvement into account, then all other things being equal, the youngest people will offer the most inaccurate SLS. People in the oldest age group are in the opposite position as regards future mortality improvement, and they may also make their decision based on more information gathered over a lifetime of observation and their own personal risk factors.

Information on personal risk factors, where it influences choice of SLS, obviously leads to being more or less likely to underestimate compared to the life table. Whether or not this is accurate cannot be determined by from this analysis, but it applies only to those influenced by those risk factors. This model predicts that those who do not use any personal information, but rely on what they think of as a normal or average lifespan for their SLS are more likely to underestimate than all others. Thus, lifespans from the Low Mortality cohort life table can be seen as overestimating compared to the population's view of normal; or rather, that 'normal' is not perceived to include the possibility of mortality improvement to the extent in the table.

That those of Asian sole ethnicity appear more likely to underestimate than other ethnicities is a puzzle. As discussed earlier, the subsample of Asian ethnicity is very small, and the result may be spurious. 
The finding that men are less likely to underestimate relative to the Low Mortality cohort table fits with the summary statistics of the gap between SLS and each life table in Table 8.6 and with the analysis of predictor variables for the gap with the period life table in Table 8.7. Women have greater odds of choosing a congruent SLS relative to overestimating to the period table compared to men. Whether explicitly done or not, the pattern of women's SLS is consistent with ignoring future mortality improvement from the period life table, while men are more likely to choose SLS consistent with better mortality than the period life table. The cohort mortality tables introduce mortality improvement which is assumed to be at a higher rate for women than men (see Table 5.1). This has the effect of widening the gender difference in the SLS gap, while resetting the benchmark higher for women. Thus women are more likely to choose an SLS that underestimates relative to the low mortality cohort table than men.

\section{Summary: underestimation a significant risk}

If the key longevity risk is underestimating relative to the low mortality cohort table, then there are some clear predictors of who is most likely to suffer that risk in the New Zealand population: females, those under age 30 and those who think their SLS is 'average' or 'normal'. Thus, a large part of the population is at risk. The odds are twice as high for females compared to males, nearly as much for the under 30s compared to older people (odds ratio 1.9) and slightly lower for those who reason their SLS on the basis of being average compared to other reasons (odds ratio 1.7). Further, those who reason on the basis of bad risk factors are nearly 8 times more likely to choose an SLS which underestimates on this definition. Whether or not the SLS responses are 
real underestimation, or reflect accurate assessment of true individual mortality risk cannot be ascertained.

Perhaps surprisingly, ethnicity and socio-economic factors are not conclusive predictors of underestimation relative to tables that do not take these factors into account. This suggests that the known factors lowering mortality on average and shortening average lifespans are generally not taken into account by individuals.

\subsection{Conclusions}

This survey has identified subjective lifespan expectations of adult New Zealanders which form similar patterns to those found in earlier American and British surveys. As in these previous surveys, New Zealanders appear to take cues for their SLS from the contemporary pattern of deaths in the population without taking likely future mortality improvement into account. Further, this study has shown that aggregate measures conceal substantial variation in SLS with potential underestimation of lifespan in the New Zealand adult population even before allowing for future mortality improvement.

New insights have emerged on how longevity expectations are formed. Simplistic models for how the population thinks about SLS are not appropriate, as there is great diversity in reasoning. Three quarters of the New Zealand adult population reason their SLS on the basis of external, given factors or none; only one quarter reason on the basis of personal risk factors which they could influence. This appears to underplay the impact on mortality risk of lifestyle and behaviour. Within the larger group, few believe their SLS is 'normal', with most either putting faith in their genes or in a 
variation of lifespan being 'out of their hands'. Therefore, information on likely future population average SLS may simply not be immediately believed by many. Moreover, reasoning for SLS appears to be less evidence-based than the implicit assumption in previous studies that individuals choose SLS with an appreciation of the mortality risk of their demographic characteristics. This research suggests reasoning, if done at all, is on a more emotional level, with levels of financial knowledge and locus of control being associated predictors.

When asked for their likely age at death, more adult New Zealanders give answers that underestimate what is considered likely in population age- and gender-specific population projections of future lifespans than give higher answers or answers close to the projections. This holds for both the official Medium Mortality cohort projections, and those considered more likely by the analysis in this thesis, the Low Mortality projections. The pattern is consistent with a general lack of appreciation that mortality is improving. Compared to the period life table which assumes no future mortality improvement, female New Zealanders underestimate lifespans on average by over two years, while males are more likely to choose SLS congruent to the table. Taking future mortality improvement into account increases the prevalence of likely underestimation in lifespan substantially. Using the medium estimate cohort table shows an underestimation of around four years for men and women. Using the cohort table with projected Low Mortality, New Zealand men underestimate their SLS on average by over five years and women by over seven years.

There are higher proportions of men compared to women at both extremes of low and high SLS, suggesting subgroups of men both over- 
pessimistic and over-optimistic; both subgroups are associated with low financial knowledge. Those under age 30 are more likely to underestimate than older people, because the effect of unanticipated mortality improvement is greater. No other demographic mortality risk factors appear salient predictors of underestimation.

Reason for choice of SLS is a predictor for high or low SLS; the risk of underestimation is strongly associated with the reason behind choosing SLS. Those who do not have a reason, or who give a reason on the basis of their SLS being 'average or normal' or who cite their personal bad mortality risk factors are more likely to give a low or very low SLS, and so are at risk of underestimating their lifespan. Those who cite good risk factors or genes or family history of lifespan are more likely to choose an SLS at the higher end, so are less likely to underestimate SLS compared to population life tables.

However, underestimation of lifespan may still exist even where people choose a higher SLS. The benefit of 'good' risk factors in extending longevity may not be fully appreciated, or those taking their parents' or grandparents' lifespan may not allow sufficiently for mortality improvement over time. Those choosing the lowest SLS may turn out to have actual lifespan in the lower range, but still have longevity risk from living longer than they expect, and from not having money saved for retirement because of low financial knowledge or low net worth. The optimists who choose the highest SLS are also more likely to have low financial knowledge, suggesting that they may be a group of confident optimists rather than accurate predictors or planners. Whether or not they turn out to live as long as they expect, their longevity risk may arise from poor financial planning for retirement. 


\section{Chapter 9: Longevity risk in New Zealand}

Longevity risk - the risk that people live longer than expected - can exist in retirement plans or in government policy through underestimation of longevity. The previous chapter showed that there is substantial underestimation of longevity by adult New Zealanders. This chapter discusses the sources of potential longevity risk in New Zealand, first in individuals' retirement planning, and second within superannuation policy. The third section attempts to quantify longevity risk in both domains.

\subsection{Potential for individual longevity risk}

"Longevity risk" for the individual is defined as the risk of living longer than expected in retirement planning, so that retirement is of longer duration than expected and personal finances in retirement become compromised. The previous chapter showed that underestimation of potential lifespans appears to exist in New Zealand but that translates into longevity risk for individuals' retirement only if potentially low lifespans are taken into account when planning for retirement. Assuming a too early retirement age can also lead to the risk of a longer than expected duration of retirement. Using further analysis from the survey described in Chapter 7 this section explores these two risks for adult New Zealanders. It describes how New Zealanders consider lifespan, retirement age and duration of retirement in their retirement plans. 


\subsubsection{How New Zealanders consider lifespan in retirement plans}

Fewer New Zealanders claim to have thought about financial planning for retirement than have thought about the age they might live to. Whereas an estimated nearly 70 per cent of the population said they had given the age they might live to some or a lot of thought, under 60 per cent said they had thought about financial planning for retirement a lot or a fair amount (55.6 per cent, weighted estimate, sample error +/-3.3 per cent; the questions were phrased slightly differently, see Table 7.5, but responses for both were collected on a 4point Likert scale). This suggests that retirement plans, including intended retirement ages, are less well considered than life plans more generally, including subjective lifespan (SLS).

Further, SLS does not seem strongly attached to retirement planning. This is despite having thought about SLS and having thought about financial planning for retirement being highly correlated $\left(\chi^{2}(1)=41.864, p<.001\right)$. Those who have at best not really thought about SLS are significantly more likely to have given financial planning for retirement a little or no thought than those who have thought about SLS. However, when asked what things need to be considered by people saving for retirement, fewer than one quarter offered unprompted a response coded as "How long they will live for in their retirement" or "Their life expectancy" (24.1 per cent, weighted estimate, sample error $+/-2.9$ per cent). This compares with an estimated 75 per cent offering responses to do with required spending in retirement, 58 per cent offering responses on current financial situation and 44 per cent offering responses on income available in retirement (Colmar Brunton 2009 p. 97). Even if a slight majority of the population have thought about financial planning for retirement, only a minority have considered SLS in that context. 
Knowing that lifespan should be considered for retirement saving was not significantly associated with having thought about SLS, or with having thought about financial planning for retirement. Indeed, the only variable significantly associated at $\mathrm{p}<.001$ was financial knowledge group $\left(\chi^{2}(2)=\right.$ 51.216). Those offering longevity as a consideration when saving for retirement were more likely to be in the High knowledge group and less likely to be in the Low knowledge group (both at $\mathrm{p}<.001$ ). Level of financial knowledge appears to be the best predictor of whether people know possible lifespan should be considered when planning saving for retirement.

\subsubsection{Intended retirement age}

Despite some lack of knowledge about planning for retirement, when asked for their likely retirement age, only around 5 per cent said they didn't know (5.3 per cent, sample error $+/-1.5$ per cent). Almost three times as many gave no response when asked for their SLS. However, an estimated 15.8 per cent of the population gave "I've already retired or I don't work" as a response to the question on likely retirement age. The analogous group of course does not exist when asking for SLS.

Excluding the latter group of non-workers, the distribution of responses to the question "At what age, if any, do you think you are most likely to retire?" was different from that for SLS (Figure 9.1). Likely retirement ages were less variable and bunched at the left of the distribution. The mode and median likely retirement age were both 65 years, the mean was 65.4 years and the standard deviation was 5.9 years, compared to a standard deviation for SLS of 10.5 years. This partly reflects that the age options on the showcards for both 
questions started at 60 and under, but the SLS question continued until "Over $100 "$, whereas the retirement age question swept up all ages over 75 into "Never, I'll carry on working".

\section{Figure 9.1: Likely retirement age, estimated percentage of the population}

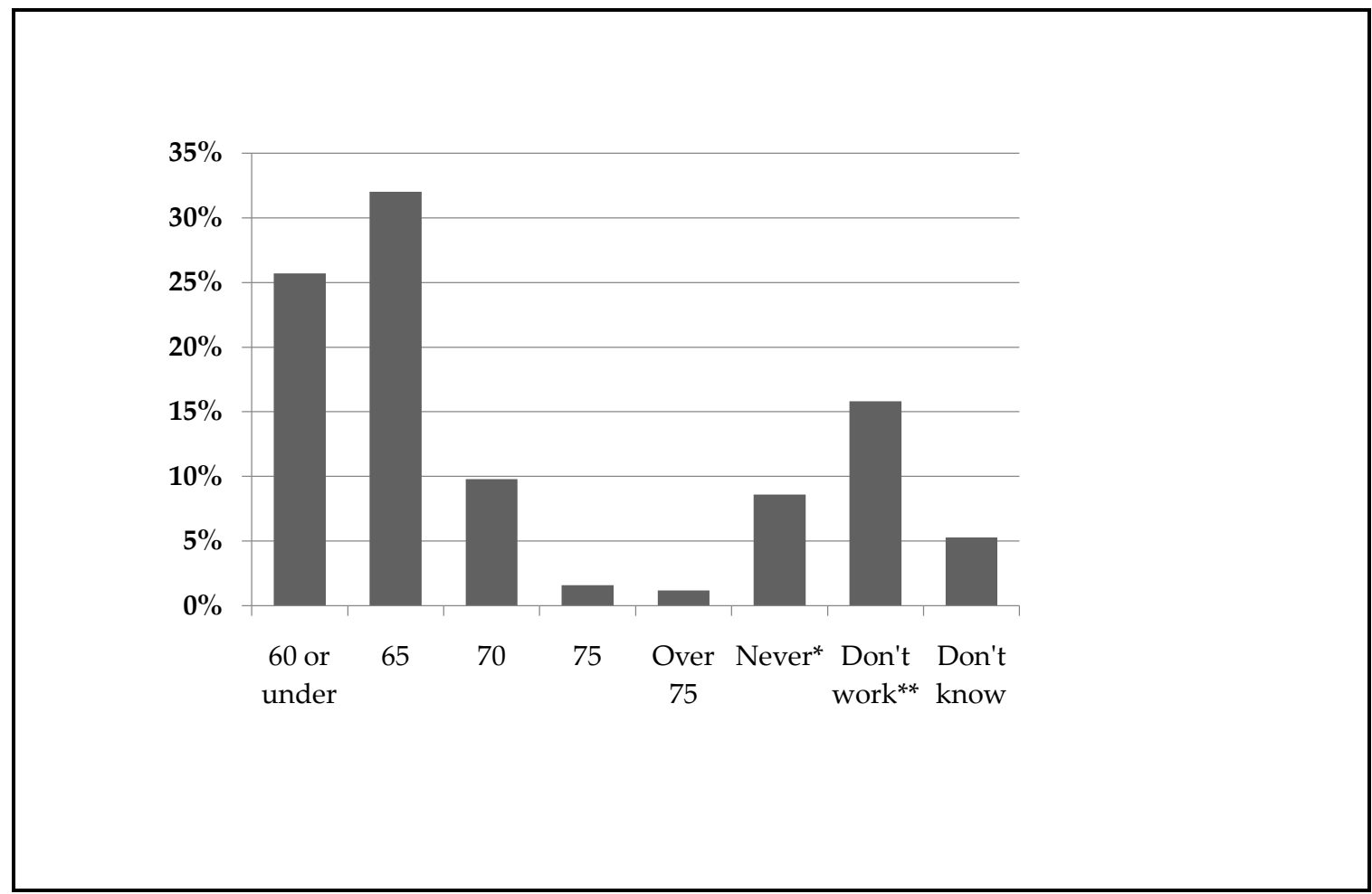

Source: Analysis of Financial Knowledge 2009 survey responses.

$\mathrm{n}=850$. *"Never, I'll carry on working". **I've already retired or I don't work".

Given that many people have not given financial planning for retirement much thought, this clustering of likely retirement age around age 65 may not always be a signal of intention, but rather of supposition that they will follow what they think of as the normal retirement age. The concentration of responses around age 65 underlies how entrenched that age is as a marker of that perceived norm of retirement. This is despite there being no legal barrier to retirement at any age and the rising participation of New Zealanders aged 65 or over in the labour force: at 17 per cent at Census 2006, the age 65 and 
over participation rate had tripled from the low in 1991 (Statistics New Zealand 2009b). The age of eligibility of the public pension is also likely to act as a marker for retirement. Since public pension began in New Zealand in 1898 , and prior to the inception of the New Zealand Superannuation (then called National Superannuation) in 1977, the eligibility age was age 65 , with a period in the mid 20th century when a reduced benefit was also available at age 60. Eligibility age was raised to age 61 effective 1992 and reached the current age of 65 in 2001 (Preston 2008). Both of the two intended retirement ages chosen by the majority of adult New Zealanders have been the ages of eligibility for the public pension within memory.

To investigate the characteristics associated with high or low likely retirement ages, the responses of those working and not already retired were split into two groups: those choosing a likely retirement age of 65 and under $(n=491)$ and those choosing one of 70 years and over (responses were collected at quinquennial ages) including in the latter group $(n=180)$ those who said "Never, I'll carry on working". Associations found in exploratory analysis (not shown) suggested that early or late likely future retirement age may be determined by age, ethnicity and socio-economic factors. These were tested more fully by logistic regression, the results of which are shown in Table 9.1. 
Table 9.1: Predictors of likely retirement age of 70 or more (including never), as compared with 65 or below

\begin{tabular}{|c|c|c|c|c|}
\hline & & $95 \% \mathrm{C}$ & for Od & Ratio \\
\hline & $\begin{array}{l}\text { Coefficient b } \\
\text { (standard error) }\end{array}$ & Lower & $\begin{array}{l}\text { Odds } \\
\text { Ratio }\end{array}$ & Upper \\
\hline $\begin{array}{l}\text { Variables included in } \\
\text { model }\end{array}$ & & & & \\
\hline Constant & $-1.267^{* * *}(0.242)$ & & & \\
\hline Age 60 and over & $1.641^{* * *}(0.343)$ & 2.637 & 5.161 & 10.102 \\
\hline Age 45-59 & $0.972^{* * *}(0.250)$ & 1.621 & 2.644 & 4.313 \\
\hline $\begin{array}{l}\text { SLS } 85 \text { and over (those that } \\
\text { gave an answer) }\end{array}$ & $0.859^{* * *}(0.203)$ & 1.586 & 2.362 & 3.517 \\
\hline Net wealth over $\$ 300,000$ & $-0.775^{* *}(0.259)$ & 0.277 & 0.461 & 0.766 \\
\hline Male & $0.488^{*}(0.203)$ & 1.094 & 1.628 & 2.424 \\
\hline NZ European sole ethnicity & $-0.560^{*}(0.226)$ & 0.367 & 0.571 & 0.890 \\
\hline [Have will] & $-0.480^{*}(0.242)$ & 0.385 & 0.619 & 0.994 \\
\hline [Asian sole ethnicity] & $-0.650 \quad(0.367)$ & 0.254 & 0.522 & 1.073 \\
\hline In High FK group & $-0.376 \quad(0.218)$ & 0.447 & 0.687 & 1.054 \\
\hline Source: Analysis of Financia & Knowledge 2009 su & rey respon & & \\
\hline Variables excluded from mo & & & & \\
\hline Each first reason for giving & S; Māori sole ethni & ty; Have $t$ & tiary ed & tion \\
\hline High personal income; Nega & ve net wealth; Age & nder 30 ar & $30-44$ & \\
\hline Note: & & & & \\
\hline $\begin{array}{l}\text { Each variable is defined as } \mathrm{s} \\
\text { combined. }\end{array}$ & ted category compa & ed to all o & er categ & \\
\hline $\begin{array}{l}\text { Backwards stepwise method } \\
\text { brackets. }\end{array}$ & ased. Forward met & d exclud & variable & square \\
\hline $\mathrm{R}^{2}=.11($ Cox and Snell), .16 & Jagelkerke). Model & $2(9)=67$ & & \\
\hline Significance level: ${ }^{*} \mathrm{p}<.05$; & $\mathrm{p}<.01 ; * * * \mathrm{p}<.001$ & & & \\
\hline
\end{tabular}

This analysis reveals that later retirement is predicted most strongly by older age, higher SLS, lower net wealth and to a lesser extent by gender. The model does not explain all of the variance in retirement age, as shown by the low $\mathrm{R}^{2}$ statistics. It is possible that an exogenous factor, such as the perception 
of age 65 as perceived normal retirement age at eligibility age for the public pension, exerts more influence on individuals' intentions than any of the variables shown here.

Nevertheless, this model suggests that intended retirement aged 70 and over (among those workers who gave an expected retirement age) is more likely for those over age 45 as compared with younger workers, and especially for those over age 60 and over. It is significantly less likely for those with net wealth over $\$ 300,000$. This pattern suggests that people become more likely to consider later retirement as they age, unless they have the wealth to consider retiring earlier. New Zealanders who give European as their sole ethnicity may be more likely to be in this financial position; it may also be the case that the norm of retiring at 65 may be more entrenched for this group. The pattern of changing intentions with increasing age and wealth implies increasing realism as retirement becomes a nearer event and wealth is more known. Declarations of intended early retirement from young people may well be turn out to be unrealistic and premature. The inverse relationship between wealth and retirement age intentions may be more of a concern from a social policy perspective. Those in higher socio-economic groups have an actuarially greater chance of a longer life than those in lower socio-economic groups. The evidenced disparity in length of intended retirement by socio-economic differential magnifies the disparity in lifespan alone.

A higher retirement age was also associated with a higher SLS, although this analysis cannot show whether high SLS leads to a higher intended retirement age, or the reverse, or whether this is a consequence of another variable. The association is logical in the sense that a longer expected lifespan 
should allow a later retirement age. It also confirms findings from international studies, notably van Solinge and Henken (2009) and Hurd et al (2003), that later retirement intentions were associated with longer expected life.

\subsubsection{Implied length of retirement}

To explore the length of retirement implied by the responses to the questions on intended retirement age and expected length of life, the gap between the two was calculated for each respondent who gave an answer to both $(n=603)$. This is most likely not the length of retirement most people are explicitly expecting; as previous analysis has indicated how few people plan their retirement, let alone those who do so with life expectancy as a consideration. The purpose of this analysis is to investigate the distribution of implicit assumptions being made. This variable is therefore called "implied length of retirement ".

Both SLS and likely retirement age were collected at quinquennial ages, so for most responses implied length of retirement was found by simple subtraction, and is itself a multiple of 5 years. At the extreme ends of the scales, an SLS of 60 or under was taken as 60, and an SLS of over 100 was taken as 105. A likely retirement age of 60 or under was taken as 59 , and one of over 75 was taken as 77, with the choice of cut off points made at smaller gaps than 5 years because of the narrowness of the retirement age choices. "Never" was taken as 77 or SLS if lower. Four cases were removed where SLS was lower than the given likely retirement age. 
The resulting implied lengths of retirement ranged from zero to 46 years. The median was 16 years, mean 16.7 years and mode 20 years. Inevitably, given the bunching at quinquennial ages and rather arbitrary choice of end values, the distribution was lumpy. However, the data fell naturally into three equally sized groups, and these tritiles, as shown in Figure 9.2 were used for further analysis.

Figure 9.2: Implied length of retirement in years, estimated percentage of the population, grouped by tritile

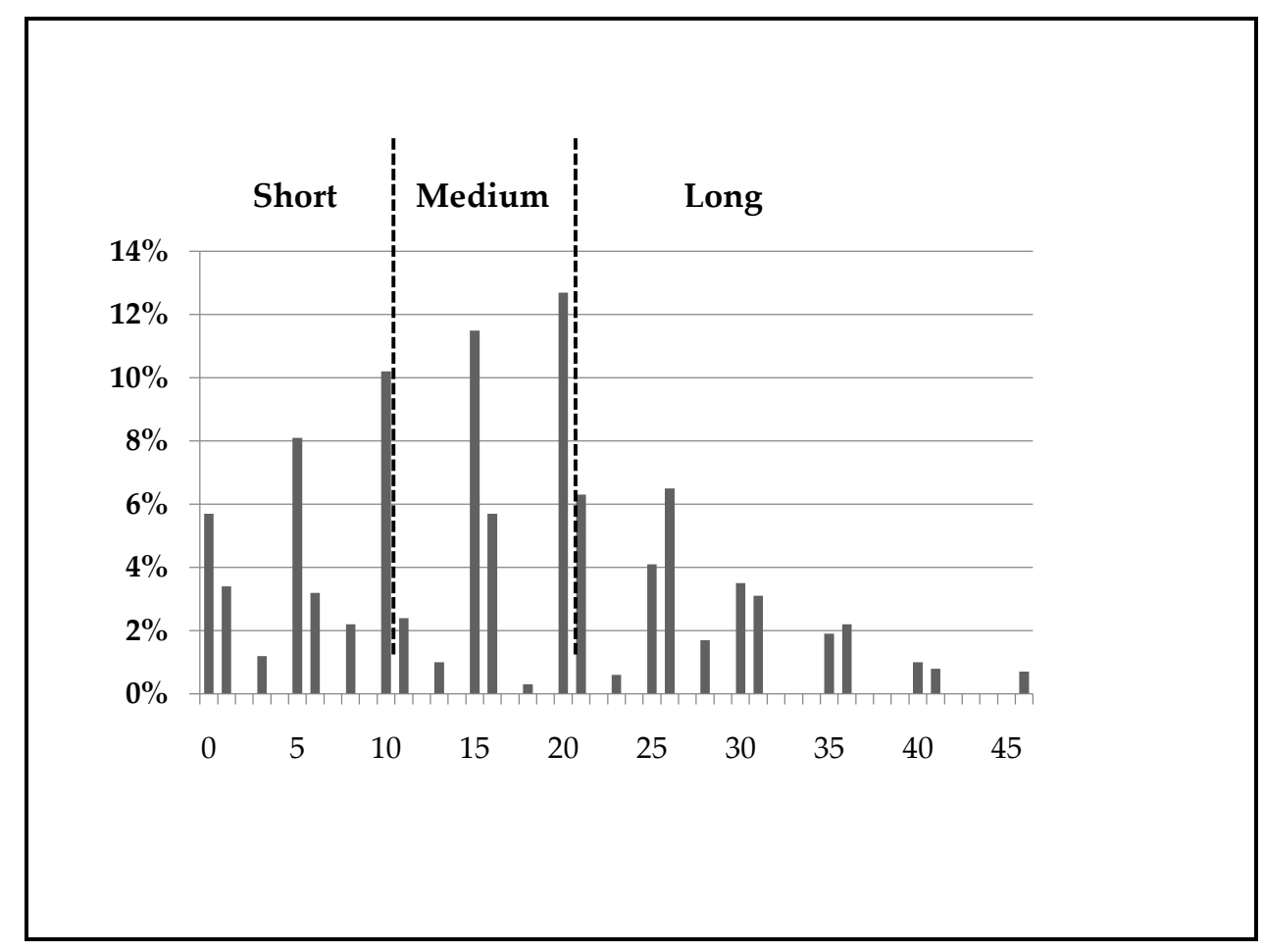

Source: Analysis of Financial Knowledge 2009 survey responses. $\mathrm{n}=603$ workers who gave an SLS.

Table 9.2 shows how each significantly associated variable is distributed by tritile of implied length of retirement. Other variables were tested but do not show significant associations. 
Table 9.2: Implied length of retirement, by tritile

\begin{tabular}{|c|c|c|c|}
\hline \multirow[b]{2}{*}{ Variable/category } & \multicolumn{3}{|c|}{ Percentage in each tritile } \\
\hline & $\begin{array}{l}\text { Short } \\
10 \text { or } \\
\text { fewer } \\
\text { years }\end{array}$ & $\begin{array}{l}\text { Medium } \\
11 \text { to } 20 \\
\text { years }\end{array}$ & $\begin{array}{l}\text { Long } \\
\text { More than } \\
20 \text { years }\end{array}$ \\
\hline Total sample, $n=603$ & 33.8 & 33.7 & 32.5 \\
\hline $\begin{array}{l}\text { Gender ** } \\
\text { Male } \\
\text { Female }\end{array}$ & $\begin{array}{l}39.2 \\
28.3\end{array}$ & $\begin{array}{l}32.8 \\
34.7\end{array}$ & $\begin{array}{l}28.0 \\
37.1\end{array}$ \\
\hline $\begin{array}{l}\text { Age split at } 30 * \\
\text { Age under } 30 \\
\text { Age } 30 \text { or more }\end{array}$ & $\begin{array}{l}38.8 \\
32.2 \\
\end{array}$ & $\begin{array}{c}24.3^{*} \\
36.8 \\
\end{array}$ & $\begin{array}{l}36.8 \\
31.0 \\
\end{array}$ \\
\hline $\begin{array}{l}\text { Personal income * } \\
\text { Up to and including } \$ 30,000 \text { pa } \\
\text { Over } \$ 30,000 \text { pa } \\
\text { Don't know or refused }\end{array}$ & $\begin{array}{l}40.3 \\
29.3 \\
24.3 \\
\end{array}$ & $\begin{array}{l}29.8 \\
35.5 \\
45.9 \\
\end{array}$ & $\begin{array}{l}29.8 \\
35.2 \\
29.7 \\
\end{array}$ \\
\hline $\begin{array}{l}\text { Net wealth *** } \\
\text { Negative } \\
0-\$ 100 \mathrm{k} \\
\$ 101 \mathrm{k}-\$ 300 \mathrm{k} \\
\$ 301 \mathrm{k}-\$ 600 \mathrm{k} \\
\$ 601 \mathrm{k}+\end{array}$ & $\begin{array}{c}45.2^{*} \\
31.3 \\
33.7 \\
28.6 \\
25.0 \\
\end{array}$ & $\begin{array}{c}18.5^{* * *} \\
34.0 \\
41.6 \\
45.5^{*} \\
36.4 \\
\end{array}$ & $\begin{array}{l}36.3 \\
34.7 \\
24.8 \\
25.9 \\
38.6 \\
\end{array}$ \\
\hline $\begin{array}{l}\text { Made a will *** } \\
\text { No or don't know } \\
\text { Yes }\end{array}$ & $\begin{array}{l}36.1 \\
31.4 \\
\end{array}$ & $\begin{array}{l}26.5^{*} \\
41.4^{*}\end{array}$ & $\begin{array}{l}37.4 \\
27.2 \\
\end{array}$ \\
\hline $\begin{array}{l}\text { How much thought about financial } \\
\text { planning for retirement? } \\
\text { A little or not at all } \\
\text { A lot or a fair amount }\end{array}$ & $\begin{array}{l}39.8 \\
28.7 \\
\end{array}$ & $\begin{array}{l}28.7 \\
38.0 \\
\end{array}$ & $\begin{array}{l}31.5 \\
33.3 \\
\end{array}$ \\
\hline $\begin{array}{l}\text { Financial Knowledge group }{ }^{* * *} \\
\text { Low } \\
\text { Middle } \\
\text { High }\end{array}$ & $\begin{array}{l}45.2^{*} \\
37.0 \\
26.0^{*}\end{array}$ & $\begin{array}{c}21.7^{* *} \\
33.8 \\
40.1 \\
\end{array}$ & $\begin{array}{l}33.1 \\
29.2 \\
33.9 \\
\end{array}$ \\
\hline $\begin{array}{l}\text { First reason for SLS } * * * \\
\text { Average/normal } \\
\text { Genes } \\
\text { Bad risk factors } \\
\text { Good risk factors } \\
\text { Other or don't know } \\
\end{array}$ & $\begin{array}{c}36.1 \\
22.9^{* *} \\
67.9^{* * *} \\
27.2 \\
43.5 \\
\end{array}$ & $\begin{array}{c}48.6^{*} \\
40.7 \\
23.2 \\
28.1 \\
22.9^{*} \\
\end{array}$ & $\begin{array}{c}15.3^{*} \\
36.4 \\
8.9^{* *} \\
44.7 \\
33.6 \\
\end{array}$ \\
\hline
\end{tabular}

Source: Analysis of Financial Knowledge 2009 survey responses.

See notes on following page. 
Notes for Table 9.2: A Pearson's chi-squared test was carried out between the variable denoted in the table columns and each variable in a subsection of the table. An asterisk next to the variable name denotes significant association between the two variables. An asterisk within a cell denotes a high standardised residual value for that category under the assumption of no association between the two variables. ${ }^{*} \mathrm{p}<.05 ;{ }^{* *} \mathrm{p}<.01 ;{ }^{* * *} \mathrm{p}<.001$.

Socio-economic status appears linked with implied length of retirement as it did with both SLS and retirement age, but is most apparent in the profile of the Short tritile. Those with negative net wealth are significantly more likely to be expecting a short retirement, as are those in the low financial knowledge group.

The pattern of association with reasons for SLS suggests that predictors of high or low SLS follow through to an implied consistent retirement age: a first reason of genes is a predictor of a high SLS and less likely to be associated with a short implied retirements; a first reason of bad risk factors is a predictor of low SLS and a short implied retirement. Other associations are less compelling. There is no obvious association with age, except that people aged under 30 are less likely to expect a mid-range length of retirement, but may expect either a short or long one. The converse is apparent with those who have made a will; this group are significantly more likely to be in the middle tritile for implied length of retirement. This pattern seems to suggest knowledge and the likelihood of reasonable intentions increasing with age and with making practical decisions related to lifespan. 
Longevity risk can occur whether implied length of retirement is short, medium or long. The financial impact of the risk appears greatest for people who expected a low SLS, but a relatively high retirement age, so are in the tritile of shortest implied length of retirement. In theory, living a number of years longer than assumed would lead to a proportionately larger savings shortfall if a short retirement were expected compared to a long retirement. Short expected lifespans may turn out to be accurate if assessments of poor mortality risk factors were correct. However, the probability of living longer than expected still exists, and, as being in this tritile is associated with having low financial knowledge and negative net worth, people in this position may

not have money saved for retirement. Further, if they have health problems or cannot find suitable work (and many in this tritile expect to work until age 70 or later), then their retirement finances may be further compromised from their implicit or explicit expectations.

\subsection{Potential for longevity risk in public pension policy}

Longevity risk in public pension policy exists if too low lifespans are assumed in the evidence used by policy makers when estimating the future cost of public pensions and in deciding on policy reform. This section considers whether this is the case, first briefly for the comparator countries used in previous mortality analysis to give some international context for the situation in New Zealand, and then focusing in more detail on the latter. 


\subsubsection{How lifespan is considered in pension policy}

Many countries have reformed pension policy over the last decade or two with increasing longevity cited as one of the main reasons for change. Raising the age of eligibility for the public pension has been the most common reform in developed economies. Further increases are planned, and applauded (OECD 2007, 2011a, b). The OECD explicitly links policy for increasing eligibility age (which it calls pensionable age) to increasing lifespans:

"By 2050, the average pensionable age in OECD countries will reach nearly 65 for both sexes: an increase of nearly 2.5 years for men and 4 years for women on 2010. However, life expectancy is projected to grow faster than these increases in pension age. Life expectancy at pensionable age is forecast to increase by about 3 years for men and 2.5 years for women between 2010 and 2050." OECD 2011b p. 21

The OECD analysis underlying this conclusion uses the projected change in period life expectancies at pensionable age from the United Nations population division, 2008 revision (OECD 2011b p. 27). Thus, it uses a measure that is likely to underestimate average age at death. If this were a basis for a policy recommendation, it would therefore contain longevity risk. The OECD does not make a specific argument for why eligibility age should keep pace with increasing life expectancy. For example, a valid policy could be to retain eligibility age in order to offer people the ability to fund greater leisure time after that age. However, the implicit rationale appears to be that increasing length in retirement (or, more precisely, time spent receiving a public pension - in many countries receipt of the pension is not contingent on 
having retired) will increase the cost of providing the public pension. The OECD does not suggest a specific cost goal, but it appears to be implicit that length of retirement should be constant rather than increasing. The absolute level of total costs could increase even if length of retirement were constant if the number of people over the eligibility age increases, and costs as a percentage of Gross Domestic Product (GDP) could increase if GDP failed to grow at the same pace as the number of pension recipients. Within any country, the precise cost goal and the importance of cost in relation to other factors shaping the structure of the public pension and economy would be a matter of public policy subject to political debate within that country. Therefore, although longevity risk is likely to exist in eligibility age policy at the level of the OECD analysis, the extent of longevity risk and the importance of increasing longevity will vary by country.

Table 9.3 compares the policy position on raising age of eligibility for the public pension in the countries used as comparators for the mortality analysis in previous chapters. This shows that only New Zealand and Canada have not yet acted to increase eligibility age beyond age 65. Thus, countries with both worse (UK, USA) and better (Australia) average lifespans than New Zealand plan to increase eligibility age to age 67 or age 68 , on differing timetables. For the same countries, Table 9.4 summarises the longevity evidence published as rationale for increasing the age of eligibility from policy makers where planned or legislated, or from other bodies where proposals have been made. 
Table 9.3: Policy position on raising age of eligibility for the public pension in selected countries

\begin{tabular}{|c|c|}
\hline & $\begin{array}{l}\text { Age of eligibility for public pension and policy } \\
\text { position as at late } 2011\end{array}$ \\
\hline Australia & $\begin{array}{l}\text { Legislation was enacted in } 2009 \text { to increase Age } \\
\text { Pension age of eligibility from age } 65 \text { to } 67 \text { at a rate of } \\
\text { six months every two years, starting } 1 \text { July } 2017 \text { and } \\
\text { finishing } 1 \text { July } 2023 \text {. } \\
\text { Affects people born } 1 \text { July } 1952 \text { and later. } \\
\text { (Australian Government 2009). }\end{array}$ \\
\hline Canada & $\begin{array}{l}\text { No legislative plans, but proposals are in debate. } \\
\text { For example, the Mowat Centre for Policy Innovation } \\
\text { published costed proposals for increasing the normal } \\
\text { age of eligibility for the Canada Pension Plan and } \\
\text { Quebec Pension Plan from age } 65 \text { to } 67 \text { at a rate of } \\
\text { two months every year starting } 2012 \text { and finishing } \\
2023 . \\
\text { Would affect people born in } 1952 \text { and later. } \\
\text { (Hering and Klassen 2011). }\end{array}$ \\
\hline New Zealand & $\begin{array}{l}\text { Commission for Financial Literacy and Retirement } \\
\text { Income (CFLRI), then called the Retirement } \\
\text { Commission, proposed an increase in New Zealand } \\
\text { Superannuation from age } 65 \text { to age } 67 \text { at a rate of two } \\
\text { months every year starting } 2020 \text { and finishing } 2033 \text {. } \\
\text { Would affect those born in } 1955 \text { and later (Retirement } \\
\text { Commissioner } 2010 \text { Table } 6.2 \text { ). } \\
\text { No action taken by Government. } \\
\text { "Prime Minister John Key said there would be } \\
\text { no change on his watch. ... He disagreed with } \\
\text { the assertion the current age had to rise. } \\
\text { "For a variety of reasons but in my view, New } \\
\text { Zealand super is sustainable at age } 65, \text {, he } \\
\text { said." } \\
\text { TVNZ } 2010\end{array}$ \\
\hline & $\begin{array}{l}\text { In November } 2011 \text { the opposition Labour party } \\
\text { adopted the CFLRI proposal as policy. }\end{array}$ \\
\hline
\end{tabular}


Table 9.3: Policy position on raising age of eligibility for the public pension in selected countries, continued

\begin{tabular}{|c|c|}
\hline & $\begin{array}{l}\text { Age of eligibility for public pension and policy } \\
\text { position as at late } 2011\end{array}$ \\
\hline $\begin{array}{l}\text { United } \\
\text { Kingdom } \\
\text { (UK) }\end{array}$ & $\begin{array}{l}\text { Up to } 5 \text { April } 2010 \text { the State Pension age (SPa) was } 60 \\
\text { for women and } 65 \text { for men. Legislation was enacted } \\
\text { in } 1995 \text { for women's SPa to increase gradually from } \\
\text { April } 2010 \text { to reach } 65 \text { in } 2020 \text {. } \\
\text { Legislation was enacted in } 2007 \text { to increase SPa for } \\
\text { both men and women to } 66 \text { between April } 2024 \text { and } \\
\text { April } 2026 \text {, to } 67 \text { between April } 2034 \text { and April } 2036 \\
\text { and to } 68 \text { from April } 2044 \text { to April } 2046 \text {. } \\
\text { Affects people born } 6 \text { April } 1959 \text { onwards. } \\
\text { The Pensions Act } 2011 \text { accelerated the increase in SPa } \\
\text { to } 65 \text { for women and to } 66 \text { for both men and women. } \\
\text { Women's SPa will increase to } 65 \text { between April } 2016 \\
\text { and November } 2018 \text {. The SPa for both men and } \\
\text { women will start to increase in December } 2018 \text { and } \\
\text { reach } 66 \text { in October } 2020 \text {. The first cohort to have SPa } \\
\text { over } 65 \text { years exactly will have been born in } \\
\text { December } 1953 \text {. } \\
\text { (PPI } 2011 \text {; UK Government } 2011 \text { ) }\end{array}$ \\
\hline $\begin{array}{l}\text { United States } \\
\text { of America } \\
\text { (USA) }\end{array}$ & $\begin{array}{l}\text { Legislation was enacted in } 1983 \text { to increase the age of } \\
\text { eligibility for unreduced Social Security retirement } \\
\text { benefits ("full or normal retirement age") from age } 65 \\
\text { to } 67 \text { over the period } 2003 \text { to } 2027 \text {, with an 11-year } \\
\text { pause during which the age will remain at } 66 . \\
\text { Affects people born in } 1938 \text { and later. } \\
\text { Social Security Online (2011) and related documents }\end{array}$ \\
\hline
\end{tabular}

Note: "Age of eligibility" is used as a general term here for the age at which full benefits are available from the main public pension. Reduced benefits may be able to be taken at earlier ages, and other benefits, sometimes with other eligibility ages, also exist in each country. 
Table 9.4: Longevity rationale invoked for proposed or actual increase in public pension eligibility age in selected countries

\begin{tabular}{|c|c|}
\hline & Longevity rationale, as published \\
\hline Australia & $\begin{array}{l}\text { "...people are living longer and spending more } \\
\text { retirement years in good health. Despite this the } \\
\text { Age Pension age has not been increased above } 65 \\
\text { years since its inception in } 1909 \text {. When the Age } \\
\text { Pension was introduced, a male retiring at age } 65 \\
\text { would have expected to spend } 11 \text { years in } \\
\text { retirement. At that time, around half of the male } \\
\text { population reached retirement age. Today over } 85 \\
\text { per cent of the male population reaches retirement } \\
\text { age and can expect to spend over } 19 \text { years in } \\
\text { retirement. } \\
\text { To respond to the long-term cost of demographic } \\
\text { change, and to reflect improvements in life } \\
\text { expectancy, the Government will progressively } \\
\text { increase the qualifying age for the Age Pension." } \\
\text { (Australian Government 2009). } \\
\text { The source is not given but the figures are } \\
\text { consistent with period life expectancy measures. }\end{array}$ \\
\hline Canada & $\begin{array}{l}\text { "Canadians now live considerably longer than } \\
\text { policy makers expected when they reformed the } \\
\text { CPP and QPP in the late 1990s. Official estimates } \\
\text { show that by 2050, men and women who retire at } \\
\text { age } 65 \text { will live, on average, until age } 87 \text { and } 89 \text {, } \\
\text { respectively. The latest projections of life } \\
\text { expectancy at age } 65 \text { in } 2050 \text { are thus } 3.0 \text { years and } \\
0.7 \text { years higher for men and women respectively } \\
\text { than those made during the last round of CPP } \\
\text { reforms in the 1990s. Moreover the latest official } \\
\text { projections very likely underestimate future } \\
\text { improvements in life expectancy..." } \\
\text { (Hering and Klassen } 2011 \text { p. } 6 \text { ). } \\
\text { The source is not given but the figures are } \\
\text { consistent with the cohort life expectancy medium } \\
\text { scenario as used in Chapter } 5 \text {. }\end{array}$ \\
\hline
\end{tabular}


Table 9.4: Longevity rationale invoked for proposed or actual increase in public pension eligibility age in selected countries, continued

\begin{tabular}{|c|c|}
\hline & Longevity rationale, as published \\
\hline New Zealand & 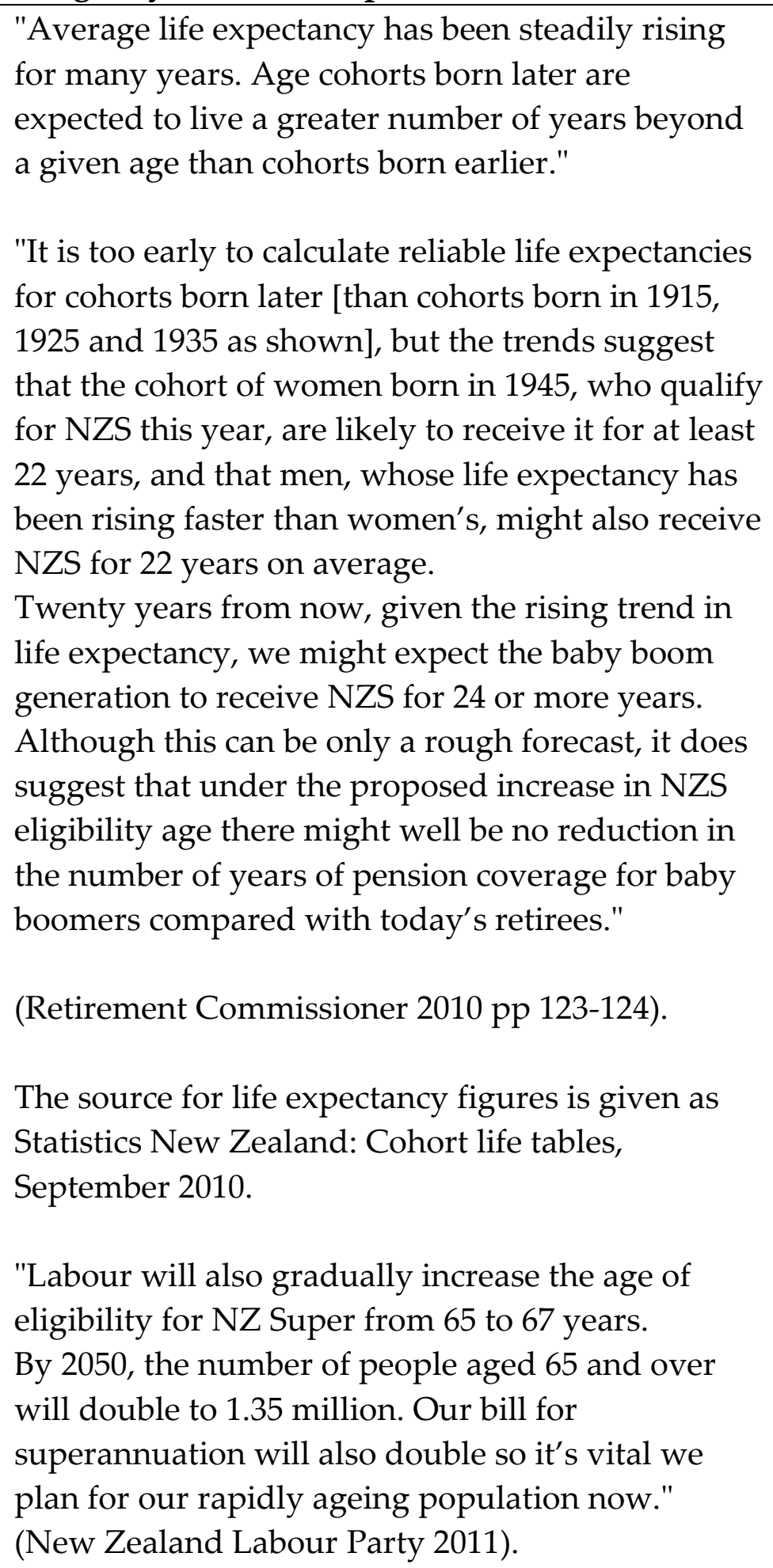 \\
\hline
\end{tabular}




\begin{tabular}{|c|c|}
\hline $\begin{array}{l}\text { United } \\
\text { Kingdom } \\
\text { (UK) }\end{array}$ & $\begin{array}{l}\text { "When the first contributory pension was } \\
\text { introduced, many people did not live long enough } \\
\text { to receive it. But more of us are living to State } \\
\text { Pension age, and receiving the State Pension for } \\
\text { longer, than at any time in our history...." } \\
\text { "The state pensions system needs to be both fair } \\
\text { and sustainable in the face of societal and } \\
\text { demographic change.... rising life expectancy } \\
\text { comes at a financial cost, which falls mainly on our } \\
\text { working-age population." } \\
\text { (DWP } 2010 \text { p.9). } \\
\text { Detailed data shown using ONS projected cohort } \\
\text { life expectancy data, including: } \\
\text { "In } 1980, \text { a woman of } 65 \text { would have been expected } \\
\text { to live to } 83 \text {, on average. Her daughter, reaching } 65 \\
\text { this year, can expect to live to } 89, \text { on average. And } \\
\text { her granddaughter, when she reaches } 65 \text { in } 2040 \text {, } \\
\text { should expect to live to } 92, \text { on average. In three } \\
\text { generations, the expected average length of life } \\
\text { after age } 65 \text { has risen by nine years...In } 1980 \text {, a } \\
\text { man received a State Pension for } 24 \text { per cent of his } \\
\text { adult life, on average....Today, a man will receive } \\
\text { it for } 32 \text { per cent of his adult life, on average. For } \\
\text { women, the proportion of adult life spent in receipt } \\
\text { of a State Pension has increased from } 36 \text { per cent in } \\
\text { 1980 to } 42 \text { per cent today, on average." } \\
\text { (DWP } 2010 \text { pp. } 10-11 \text { ). }\end{array}$ \\
\hline $\begin{array}{l}\text { United States } \\
\text { of America } \\
\text { (USA) }\end{array}$ & $\begin{array}{l}\text { "Congress cited improvements in the health of } \\
\text { older people and increases in average life } \\
\text { expectancy as primary reasons for increasing the } \\
\text { normal retirement age ... Since the program first } \\
\text { began paying monthly Social Security benefits in } \\
1940 \text { the average life expectancy for men reaching } \\
\text { age } 65 \text { has increased nearly } 4 \text { years to age 81; for } \\
\text { women reaching age } 65 \text {, their average life } \\
\text { expectancy has increased nearly } 6 \text { years to age } 84 . " \\
\text { (Social Security Online 2011). } \\
\text { The source is not given but the figures are } \\
\text { consistent with period life expectancy measures. }\end{array}$ \\
\hline
\end{tabular}


In public documents, the rationale for reform is often summarised and less than explicit, but a shared theme of response to increasing cost from increasing longevity is apparent. Of the countries where increases in eligibility age are planned by government, only the UK uses detailed future cohort life expectancies for all current ages as evidence of expected increases in average lifespans in future, but only a single scenario - the medium estimate as labelled in Chapter 5 - is used. Australia and the USA invoked only evidence of past increases in period life expectancy as argument for increasing eligibility age. For the two countries where independent bodies have made proposals for reform - New Zealand and Canada - projected cohort life expectancies are mentioned even if not referenced as such. In Canada projections further into the future are used to cover more of the current population and the possibility that these are underestimates of likely future lifespans is referred to. The New Zealand political debate, though limited, is in terms of the increasing cost from the number of people reaching the age of eligibility. This is an aggregate cost view, ignoring the impact, including cost, on individual lifespans.

Even if an explicit cost goal of legislation to increase eligibility age is not stated, the cost implication of plans is published and cost is referred to in general as one driver for change. If the cost of the public pension started to increase unexpectedly as a result of average lifespan underestimate, it may be possible to change eligibility age or other parameters within the policy structure at short notice to compensate. Therefore, it is not necessarily the case that longevity risk of this type will always lead to higher cost of the public pension than planned for. However, it would mean that eligibility age is not being raised fast enough to meet whatever the implicit cost or other goals were at the time of reform, which is usually some years before the increase in 
eligibility age begins. This risk was made explicit in the case of the UK when a new government used the rationale for longevity increasing faster than expected to propose an acceleration of the timetable for the increase to age 66 only three years after the timetable had been set:

"The Pensions Act 2007 legislated for increases in State Pension age. Under the Act, the State Pension age was planned to rise for men and women to 66 by 2026, 67 by 2036, and 68 by 2046. ... Even since this timetable was set, official projections for average life expectancy at 65 in 2026 have gone up an extra 1.5 years for men, and 1.6 years for women...

... tomorrow's pensioners will spend an even greater part of their adult life in receipt of a State Pension than was thought in 2007. No responsible government can afford to ignore the challenges of increasing longevity. It is crucial for both financial sustainability and fairness to each generation that the state pensions system reflects how much longer we are living. To ensure this, the Government has reviewed the timing of the increase in State Pension age to 66." DWP (2010) p. 9 and p. 15.

Public discourse about eligibility age policy reform may be the only way in which individuals are exposed to data about expected average lifespans. If that data is then used in individuals' retirement planning then it has a direct consequence for individual longevity risk. Of these countries, governments in Australia and the US did not use the opportunity of eligibility age reform to give information about future likely average lifespans. The UK government 
has used the best available source of future projected cohort life expectancies extensively. However, each time it uses only the medium estimate without referencing the potential for longer (or shorter) average lifespans than the medium scenario provides. For example a database published by the government department responsible for pension policy of cohort life expectancies at age 65 for cohorts reaching that age from 1951 to 2058, from projections dating from 1983 to 2008, shows only the principal projection results from the statistical or actuarial agency (DWP 2011), yet the same source details the consistent underestimation of the projections over time. The policy debates in both Canada and New Zealand have been narrow by comparison, far from a full public debate led by government. It is therefore unlikely that individuals have been able to discern good estimates of likely future lifespans, or the uncertainty involved, from the available public discourse.

Table 9.4 shows that the longevity rationale for each country's eligibility age reform or proposal has been made with a variety of parameters to describe potential average lifespans. Very few parameters are likely to be absorbed by the public and hopefully used in their own retirement planning. The most useful parameters for this purpose would intuitively be likely average duration of retirement or likely average total lifespans. Because more people think about lifespan than retirement age, it is likely that a single figure of a likely future average lifespan would be the most likely to be remembered. A range of plausible estimates for this figure could be useful to show that there is uncertainty and therefore demonstrate the need for caution in planning. None of the debates in the countries shown reach an ideal of communicating this parameter simply and memorably, quite apart from whether the best future estimate of the parameter is used. 


\subsubsection{Longevity in New Zealand's superannuation policy}

The previous section identified three potential contributions to longevity risk in New Zealand from policy for the public pension, New Zealand Superannuation (NZS). First, New Zealand is unusual among the comparator countries for not planning to increase eligibility age even though average lifespans in New Zealand are at higher levels and are increasing faster than countries that are increasing eligibility age beyond the current age of eligibility age for NZS of 65. Despite the fast improvement in mortality in New Zealand, relative to the other countries considered, even the CFLRI proposal for reform of eligibility age suggests a later increase than planned or proposed elsewhere. In declining to raise eligibility age, a political judgement is being made that the future cost of NZS is affordable and the savings to be made from increasing eligibility age could not be better used elsewhere. This policy decision means that the cost of New Zealand Superannuation is likely to increase faster than the cost of other public pensions where eligibility age is increasing because of increasing longevity.

Second, where an increase to the eligibility age has been proposed, the evidence invoked has been past or near-term cohort life expectancies, even though estimates of cohort life expectancies projected further into the future are available on request to Statistics New Zealand. Therefore, it would be possible to use future estimates of lifespan, on the Low and Medium Mortality scenarios. Although there is uncertainty in these future estimates - "It is too early to calculate reliable life expectancies for cohorts born later" as the Retirement Commissioner put it (2010 p. 124) - a discussion of that very uncertainty itself carries an important message for retirement or policy 
planning: that using a single conservative estimate carries a risk of future financial loss.

Estimates of future mortality rates consistent with future cohort life expectancies are already used within models of the future fiscal cost of New Zealand Superannuation, whether to explore the consequences of policy change such as increasing eligibility age or for the regular use of the Long Term Fiscal Model for cost estimates on current policy (Rodway 2010; Rodway and Wilson 2006; The Treasury 2006, 2009). Generally, however, only the medium scenario for mortality is used. Even if other scenarios are used they are not reported on prominently but rather as sensitivity tests on the main case. Therefore the future cost of NZS as most prominently reported from the results of the model will be underestimated compared to the results from using the low mortality variant. Thus longevity risk is introduced into policy planning from the use of a single, conservative, scenario for longevity. Further, the reporting of the results of these models is not an effective forum for giving public information on likely future lifespans because their purpose is to estimate the fiscal cost of NZS, which depends on many other assumed parameters.

Third, there are consequences from public policy on New Zealand Superannuation for longevity risk in individual retirement planning. Opportunities to give individual New Zealanders' information about their potential lifespans are limited because the public debate about eligibility age is narrow and late. In other countries, such a debate has provided a public forum for data on increasing lifespans and the implications therein to be revealed, explained and discussed. A single helpful measure of future average 
lifespans is not obvious in New Zealand's public discourse. The political party with a policy to increase age of eligibility uses the same rationale highlighted in the media release on the original proposal from the 2010 Retirement Commissioner's Review of Retirement Income Policy: the increasing number of superannuitants from the "baby boomer" generation (CFLRI 2010). The message of increasing lifespans was subsumed into the detail of the report in which there was only a tentative reference to the possibility the baby boomer generation might receive NZS for 24 or more years. This number is critically important for individual retirement planning, but not emphasised as such.

Further risks to individual retirement planning accrue from retaining New Zealand Superannuation eligibility age at 65. A low estimate of individual lifespan is associated in New Zealanders' minds with a low retirement age, with eligibility age for New Zealand Superannuation appearing as a critical factor influencing retirement age intentions (Figure 9.1). Retaining eligibility age at 65 is therefore likely to retain age 65 as the modal intended retirement age of New Zealanders. Additionally, eligibility age for New Zealand Superannuation is also the age at which KiwiSaver funds become available. Therefore, as long as an eligibility age of 65 is retained, so the basis for most New Zealanders for financial retirement planning is likely to be retirement at age 65 . The public policy position of no change to eligibility at age 65 fails to give the public the message that longevity is increasing. It does not trigger any reason for individuals to increase their explicit or implicit assumptions about retirement age or age at death.

Policy debate need not be the only source of information about potential average lifespan or appropriate or normal retirement age. However, in New 
Zealand it is the main contender. KiwiSaver is the main retirement savings vehicle for the adult population; around half of those eligible for KiwiSaver aged 18 and over are members (computed from IRD KiwiSaver membership statistics as at end April 2011 and Statistics New Zealand population estimates March 2011 quarter). Other retirement plans outside of KiwiSaver tend to make the same assumption for eligibility age, that is, they follow New Zealand Superannuation eligibility age. Illustrations of future retirement benefits from an adviser will also use an assumption of likely lifespans. The most prominent widely available calculator promoted so that New Zealanders can estimate the savings needed for their own retirement exists on the Sorted website (www.sorted.org.nz), from the Commission for Financial Literacy and Retirement Income (CFLRI). An individual using this calculator has the opportunity to input his or her own assumptions for both retirement age and age at death, but defaults are provided. Behavioural economic theory suggests these defaults would be most often used (for example, Madrian 2009). The default retirement age is age 65 . The definition or source of the default ages at death are not stated, but they are age- and gender-specific and slightly lower than period life expectancies at retirement age (see later in Table 9.5). Not all New Zealanders will have used CFLRI's retirement savings calculator. Around a third of New Zealanders are estimated to have ever used Sorted material, including booklets and seminars as well as the website, which contains many other calculators (CFLRI 2011 p. 4). Individuals may have alternatively used similar calculators with a financial advisor or policy salesman, and these may in fact rely on the Sorted calculator. Whether by setting official estimates for use in calculators such as these, or by implanting key assumptions through the use of headline numbers on retirement age and potential lifespans, the parameters used in policy and discussed in policy 
debate, and data supplied by Statistics New Zealand which in turn is used in policy debates, can all extend a strong influence on the assumptions made in individuals' retirement planning.

\subsection{Quantifying longevity risk in New Zealand}

Do the contributions to potential longevity risk described in previous sections actually constitute significant longevity risk in New Zealand's policy planning or significant longevity risk in New Zealanders' retirement planning? This section addresses this question by quantifying the effect of longevity risk in these two domains.

\subsubsection{Longevity risk in policy}

Failure to increase the age of eligibility for New Zealand Superannuation (NZS) in itself invites greater exposure to longevity risk. An unchanged eligibility age means a greater change to NZS eligibility age or other parameter would be required to mitigate the increase in future cost of NZS due to future increases in longevity than if a schedule of future increases in eligibility age were planned. Further, the political judgement which currently accepts the future fiscal cost of NZS may make a different policy decision if future costs were estimated to be higher as a result of using assumptions on mortality improving faster than Statistics New Zealand's medium scenario. Treasury does not publish full up to date cost scenarios for NZS using anything other than the medium scenario. Therefore it is difficult to gauge the extent of longevity risk as a result of conservatism in the choice of mortality assumptions. However, a recent illustration is available from Treasury work 
to estimate the cost savings from raising eligibility age (Rodway 2010). This is the source for what follows in this section.

The base case for the gross of tax fiscal cost of New Zealand Superannuation was estimated to be 4.4 per cent of Gross Domestic Product (GDP) in 2010, rising to 6.9 per cent in 2035 and 7.9 per cent in 2060. Among the many demographic and economic assumptions this calculation requires, the medium scenario for mortality from Statistics New Zealand was used. The cost in 2035 was estimated to increase by 0.3 percentage points, to 7.1 per cent of GDP, and by 0.7 percentage points, to 8.6 per cent of GDP in 2060, if mortality instead followed Statistics New Zealand's Low Mortality scenario (Rodway 2010 p. 23). Thus, if mortality did decrease on what this thesis anticipates is plausible, and no other changes to policy were made, then the fiscal cost of NZS would be higher by less than one tenth over the next five decades. While this may not be deemed highly significant, further improvements in mortality would of course make more of a fiscal impact. The Very High Life Expectancy scenario (where period life expectancy at birth is set arbitrarily to 95 years in 2060) was estimated to increase the gross cost of NZS in 2060 to 9.7 per cent of GDP. Given that cohort life expectancy at birth is already within five years of that figure for females, it is not outside the bounds of possibility that such improvement could occur over that timeframe.

Although the actual cost of mortality proving to be on the Low Mortality scenario does not look in isolation to be highly significant, some context can be provided for what policy change would be required to mitigate the cost. The reduction in cost under the medium scenario if eligibility age were increased from age 65 to age 67 on the CFLRI's proposed timetable detailed in Table 9.3 
is estimated to be 0.8 percentage points of GDP, and larger savings would be made earlier than the additional cost would be on the lighter mortality assumption (Rodway 2010 p. 10). Therefore, to mitigate the longevity risk resulting from a conservative choice of mortality assumptions in cost modelling requires a policy that provides as much cost saving as the CFLRI's proposed increase in eligibility age. This policy appears to be currently (late 2011) politically impossible to achieve. From this point of view the longevity risk in current New Zealand Superannuation policy is significant. The risk will only grow as lifespans continue to increase, and if lifespans start to lengthen by even more than the Low Mortality scenario suggests, while plans to increase eligibility age continue to be avoided.

\subsubsection{Longevity risk in individual retirement plans}

In addition to the longevity risk from failure to address increasing longevity within New Zealand Superannuation policy, the lack of signalling on rationale for increasing eligibility age is likely to lead to increased longevity risk for individual New Zealanders. As discussed above, eligibility age is a signal for retirement age and is the default age for planning when KiwiSaver funds become available. An increasing eligibility age is therefore likely to indicate an expectation of increasing intended retirement age, which has a beneficial effect on mitigating financial risk in individual retirement planning. Further, the public discourse around the longevity rationale for policy reform to increase eligibility age for New Zealand Superannuation would offer the opportunity to inform the public of plausible future lifespans using cohort life expectancy measures, which should mitigate longevity risk from using poor assumptions of likely age at death. 
This section explores the size of longevity risk in individual plans from two public policy messages: the effect on intended retirement age from the age of eligibility for New Zealand Superannuation, and the effect on subjective lifespan (SLS) from using lifespan data in public messages that underestimates the actual likely future lifespans. This analysis summarises previous work by drawing on Chapter 3 for the theoretical rationale for using cohort life expectancy rather than the more commonly used period measure, on Chapters 4 to 6 for the conclusion that the Low Mortality variant of the Statistics New Zealand projections is more plausible than the more commonly used medium estimate, on Chapters 7 to 8 for data on adult New Zealanders' SLS and on this Chapter 9 for the associations between SLS, age of eligibility for New Zealand Superannuation and intended retirement age.

Having less money than expected in retirement can arise from a number of reasons other than underestimating lifespan. Under the lifecycle savings hypothesis, individuals rationally work out how much income they will need in retirement on top of the public pension they will receive, and then save regularly to provide that excess income making assumptions for the rate of return net of costs they will achieve on their savings and the rate at which they will be able to draw down or annuitise their savings in retirement. In this rational model, there is scope for lower retirement income than expected through contributions to savings not being made as planned, public pension being less than expected through policy change, lower than expected returns on savings or worse than expected annuitisation rates. Further, retirement income may be lower than implicit expectations by those individuals who did not make retirement plans because their expectations were irrational or unrealistic. 
The impact of longevity risk on retirement income turning out lower than expected can be illustrated in the rational case by computing the financial effect of changing lifespan assumption in the lifecycle savings model. By also making retirement age intention a variable parameter, the effect of the links between the two parameters that determine length of retirement can be explored. These computations were carried out using a simple publicly available calculator based on the lifecycle savings model from the Sorted website. Results are in Table 9.6. The analysis is illustrated using one example individual born in 1960, so in 2009 was aged 49 which is a reasonable estimate for the typical age of retirement planning. This analysis computes the monthly savings required to reach a defined retirement income objective given a set of assumptions including retirement age intention and age at death. For this analysis, the latter two assumptions vary and other assumptions, including retirement income objective, are held constant at the CFLRI default, or omitted where unnecessary.

The assumptions for typical age at death likely to be used in a financial planning exercise were selected from the distribution of New Zealanders' subjective lifespans (SLS) revealed in the previous chapter, from potential signals from public commentary and from the (age-specific) recommendations from the Sorted website. The potential actual ages at death were taken from age-specific actual or projected mortality data as detailed. Table 9.5 summarises the age at death assumptions used and their sources. 
Table 9.5 shows that the contribution to underestimation of lifespans from using a flawed measure - period as opposed to cohort - is greater than the contribution from using a more plausible assumption for future mortality than the almost universally used medium estimate. Assuming survival to the age of retirement intention (that is using period life expectancy at retirement age) improves on assuming the flawed period life expectancy at birth measure by adding four years for females and five for males. Further improvement of the same amount is added by using the year of birth-specific cohort measure on the plausible scenario, but all but one of these years comes from the change from period to cohort measure, rather than from the choice of scenario of future mortality improvement. 
Table 9.5: Age at death assumptions that may be used by, or are available to New Zealanders

\begin{tabular}{|c|c|c|c|}
\hline \multirow{2}{*}{$\begin{array}{l}\text { Message } \\
\text { source }\end{array}$} & \multirow[b]{2}{*}{ Data source } & \multicolumn{2}{|c|}{ Lifespan in years } \\
\hline & & Female & Male \\
\hline \multirow{2}{*}{$\begin{array}{l}\text { Assumptions } \\
\text { made in New } \\
\text { Zealand adult } \\
\text { population }\end{array}$} & Modal SLS & 85 & 75 \\
\hline & Median SLS & 85 & 80 \\
\hline CFLRI & $\begin{array}{l}\text { Default in retirement } \\
\text { savings calculator }\end{array}$ & 85 & $81 / 82$ \\
\hline $\begin{array}{l}\text { Commonly } \\
\text { used in public } \\
\text { commentary } \\
\text { (see Table 3.1) }\end{array}$ & $\begin{array}{l}\text { Period life expectancy at } \\
\text { birth }\end{array}$ & 82 & 78 \\
\hline $\begin{array}{l}\text { Improving } \\
\text { above by } \\
\text { using age at } \\
\text { retirement }\end{array}$ & $\begin{array}{l}\text { Period life expectancy at } \\
\text { age } 60 / 65 / 67\end{array}$ & $85 / 86 / 86$ & $82 / 83 / 83$ \\
\hline $\begin{array}{l}\text { Modal age at } \\
\text { death }\end{array}$ & Period life table & 89 & 86 \\
\hline $\begin{array}{l}\text { Cohort } \\
\text { measure } \\
\text { (most realistic } \\
\text { lifespan } \\
\text { measure) }\end{array}$ & $\begin{array}{l}\text { Cohort (born 1960), life } \\
\text { expectancy at age } 65 \\
\text { medium mortality cohort } \\
\text { table }\end{array}$ & 89 & 87 \\
\hline $\begin{array}{l}\text { Cohort } \\
\text { measure using } \\
\text { more } \\
\text { plausible } \\
\text { mortality } \\
\text { improvement } \\
\text { assumption }\end{array}$ & $\begin{array}{l}\text { Cohort life expectancy at } \\
\text { age } 65 \text { (born 1960), Low } \\
\text { Mortality cohort table }\end{array}$ & 90 & 88 \\
\hline $\begin{array}{l}\text { High current } \\
\text { estimate to } \\
\text { illustrate } \\
\text { uncertainty }\end{array}$ & $\begin{array}{l}\text { 90th percentile of age of } \\
\text { actual deaths, } 2009\end{array}$ & 92 & 89 \\
\hline
\end{tabular}

See notes on following page. 
Notes for Table 9.5:

1. For modal and median SLS see Table 8.1. These are the mode and median for the New Zealand adult population as surveyed, not specific to this illustrative individual.

2. 'CFLRI' ages at death are the retirement age-specific default assumptions in the calculator. They are described as "Your expected lifetime is the age at which an average person could be expected to live to if you reach your retirement age", but source is not given. Female default is the same for retirement age 60 and 65; male varies as shown. Note that these figures are lower than the approximate 24 years after age 65 the Retirement Commissioner's 2010 Review of Retirement Income Policy used for the possible lifespan of the baby boomer generation, as part-rationale for proposing an increase in age of eligibility for New Zealand Superannuation (see section 9.2.2).

3. Period data from Statistics New Zealand published 2005-7 complete life table (the most recent complete life table as at 2009).

4. Cohort mortality table ages at deaths are expected lifespan from age 65, M65 $=65+\mathrm{e}_{65}$ for cohorts born in 1960, consistent with latest available 2009-base projections (data provided by Statistics New Zealand November 2009). Further improvement to the model should be made by using cohort life expectancy from the actual retirement age if different from 65. However, this data is not available. It should not affect the results significantly as lifespan estimates do not change by a significant amount within a low number of years, as shown by the period life table equivalents in this table. The effect of changing eligibility age of retirement from age 65 to 60 or 67 using this age at death assumption is illustrated in Table 9.6.

5. 90th percentile age at death from distribution of actual deaths in 2009, from Statistics New Zealand data

6. All life expectancy measures rounded to nearest integer. 
Modal age at death from the latest period table adds further improvement to the period life expectancy measure, getting close to cohort life expectancy. It is therefore a useful proxy for cohort lifespan at this age, and could be used if full cohort tables were not available. To introduce greater conservatism, or a margin for the possibility of further mortality improvement, higher age at death assumptions could be used. For example, the 90th percentile of the current distribution of ages at death would increase the assumption a further one or two years. Advice on age at death assumption is important as the SLS of the adult New Zealand population underestimates likely actual age at death. The advice available from the CFLRI also underestimates. Because the definition of the measure is so important for getting close to a likely age at death measure, it is important that public information on likely ages at death does use theoretically accurate measures. 
Table 9.6: Sizing the effect of longevity risk on retirement plans

\begin{tabular}{|c|c|c|c|c|c|c|}
\hline & \multicolumn{6}{|c|}{$\begin{array}{l}\text { Monthly savings required, in dollars, to meet } \\
\text { retirement income objective of } 70 \text { per cent of pre- } \\
\text { retirement income using assumption for age at } \\
\text { death and other assumptions as in notes }\end{array}$} \\
\hline & Female & & & Male & & \\
\hline $\begin{array}{l}\text { at death } \\
\text { assumption }\end{array}$ & $\begin{array}{c}\text { Retire } \\
\text { at } 60\end{array}$ & $\begin{array}{c}\text { Retire } \\
\text { at } 65\end{array}$ & $\begin{array}{l}\text { Retire } \\
\text { at } 67\end{array}$ & $\begin{array}{c}\text { Retire } \\
\text { at } 60\end{array}$ & $\begin{array}{l}\text { Retire } \\
\text { at } 65\end{array}$ & $\begin{array}{c}\text { Retire } \\
\text { at } 67\end{array}$ \\
\hline Modal SLS & 1,496 & 415 & 178 & 1,248 & 244 & 24 \\
\hline Median SLS & 1,496 & 415 & 178 & 1,383 & 337 & 108 \\
\hline CFLRI & 1,496 & 415 & 178 & 1,407 & 370 & 137 \\
\hline $\begin{array}{l}\text { Period life } \\
\text { expectancy at } \\
\text { birth }\end{array}$ & 1,431 & 370 & 137 & 1,332 & 302 & 76 \\
\hline $\begin{array}{l}\text { Period life } \\
\text { expectancy at } \\
\text { age } 60 / 65 / 67\end{array}$ & 1,496 & 429 & 190 & 1,431 & 385 & 151 \\
\hline $\begin{array}{l}\text { Modal age at } \\
\text { death }\end{array}$ & 1,573 & 468 & 226 & 1,530 & 432 & 192 \\
\hline $\begin{array}{l}\text { Medium } \\
\text { mortality } \\
\text { cohort lifespan } \\
\text { at age } 65\end{array}$ & 1,573 & 468 & 226 & 1,536 & 442 & 203 \\
\hline $\begin{array}{l}\text { Low mortality } \\
\text { cohort lifespan } \\
\text { at age } 65\end{array}$ & 1,591 & 480 & 237 & 1,555 & 455 & 214 \\
\hline $\begin{array}{l}\text { 90th percentile } \\
\text { of age of actual } \\
\text { deaths, } 2009\end{array}$ & 1,624 & 503 & 258 & 1,573 & 468 & 226 \\
\hline
\end{tabular}

See notes on following page. 
Notes to Table 9.6:

1. Calculations from Comprehensive Retirement calculator on Sorted website www.sorted.org.nz performed May 2011.

2. Illustrative case is a single person, born January 1960 with an annual income of \$40,000 (approximately median wages and salaries, New Zealand Income Survey: June 2009, Table 12, Statistics New Zealand). Changing age of individual changes the amount needed to be saved but not the proportional change in saving needed for changes in age at death.

3. The calculator default assumptions are used as at time of carrying out calculation: total retirement income objective is 70 per cent of pre-retirement pre-tax income after tax, New Zealand Superannuation of $\$ 17,676$ per annum will be available from age 65 and net real rate of return from savings is 3.6 per cent (for a 17.5 per cent tax payer in a Portfolio Investment Entity). Results are in current dollars. Other assumptions made within the calculator not stated. No additional investment lump sums, housing assets or mortgages were included in the calculation in order to focus only on the change in savings required when assumed age at death changes.

4. Monthly savings computed are in dollars from age 50 to the assumed age of retirement. For retirement at age 67, NZS payments from age 65 are assumed to be accumulated and used to contribute towards retirement income.

5. See also notes to Table 9.5.

Looking first at the example woman intending to retire at age 65, the CFLRI's recommendation for expected age at death is the same as the model and median SLS found in the New Zealand adult population. If retirement did take place at age 65, but age at death turned out to be that predicted by the average from the Medium Mortality cohort table, then monthly savings towards retirement should have been $\$ 468$ rather than $\$ 415$. For an unexpected increase in age at death of four years, savings would fall short by 11 per cent. If age at death turned out to be one year later at the average from the low mortality cohort table, or a further year later at the 90th percentile of New Zealand ages at death, then the shortfall would be 14 per cent or 17 per 
cent respectively. For the example man, the CFLRI's recommendation for expected age at death is higher than the modal and median SLS found for the New Zealand adult population. To take the extreme case shown in the table, a man expecting to die at the modal SLS of 75, but who actually turned out to live to the 90th percentile of actual deaths at age 89 , would have a shortfall in retirement savings of 21 per cent if he intended to and did retire at age 60 or a shortfall of 48 per cent with retirement at age 65 . Table 9.6 confirms that a greater contribution to longevity risk comes from using a period indicator for lifespan instead of a cohort indicator than by changing mortality improvement scenario in the cohort indicator. This result holds for individuals born ten years earlier or later than the example shown here (calculations not shown).

Retiring later makes a large difference to retirement finances. Most New Zealanders choose when asked a retirement age assumption of either 65 rather than 60 (Figure 9.1). This choice has a greater impact in required savings than any change in assumption in age at death illustrated in Table 9.6 for a given intended retirement age. Retiring later increases the number of years when savings could be made and reduces the number of years that retirement funds have to cover income needs. An increase in retirement age to age 67 from age 65 cuts the amount of savings required on any of the age at death assumptions by at least half. For some New Zealanders, a retirement age of 67 may turn out to be realistic than an increase in regular saving to meet the same retirement income goal.

The association between SLS and intended retirement age (Table 9.1) means that an expectation of a lower than likely actual lifespan may have a double negative effect on retirement finances: by not saving enough for likely 
length of retirement and by starting retirement too early. However the sensitivity to age at death assumption, and therefore the effect of longevity risk, increases with higher retirement age. The quantum of risk for New Zealanders in financial planning for retirement from a low intended retirement age is greater than longevity risk from underestimating likely age at death, but the remedy of a higher retirement age intention brings with it a greater significance of longevity risk.

New Zealand Superannuation is available as the first tier of income, so a shortfall of retirement savings should not mean absolute poverty for most. Further, for any individual facing a shortfall in savings, the option may exist of retiring later than expected. Thus, longevity risk is present in New Zealanders' individual retirement plans, but may not be financially significant for all. There is an additional, linked, and likely more significant risk from intending to retire too early. The financial impact may be able to be mitigated by saving more or working longer than intended. However, in some cases, the financial impact could be severely negative, and mitigating actions may not be available when they are needed. Therefore, even if longevity risk is not always significant for individuals, addressing the risks of longer than intended retirement would be of benefit to New Zealanders. The natural forum for theoretically correct and plausible measures of likely future lifespans that policy debate around eligibility age for New Zealand Superannuation would provide would help to address such risks. 


\subsection{Conclusions}

New Zealanders think less about financial planning for retirement than about how long they are going to live. Expectations for retirement age are concentrated around age 65, whereas expectations for age at death cover a wider range. Few people appear to have thought about length of retirement even if they have a financial plan for retirement. The lengths of retirement people implicitly expect vary widely. Individual New Zealanders suffer longevity risk in their own retirement planning when they assume implausibly low age at death; and the financial risk is often exacerbated by low intended retirement ages.

Because socio-economic position is positively associated with subjective lifespan but inversely related to retirement intentions, socio-economic disparities in implied length of retirement are magnified. People in higher socio-economic groups are more likely to expect to retire at 65 or earlier, and, probably relying on genes or family history, expect a longer than median lifespan and, implicitly, a retirement that is at least ten years or more. People in lower socio-economic groups are more likely to expect to work until 70 or beyond, and perhaps through lack of knowledge, assume a low SLS, so that implied length of retirement is likely to be short; less than ten years. Longevity risk exists whatever the implied length of retirement, but seems greatest for people who expect both a short retirement and a short lifespan. The risk of having lower than expected retirement income will be compounded for people in this group who find they are not able to correct their early retirement intention when they need to by continuing to work in later life. 
Longevity risk within New Zealand's public pension policy is greater than that in those of the British settler countries used as comparators for this thesis which have started to raise eligibility age for the main public pension (USA) or have plans to do so (Australia, UK). This is because there are no plans to increase eligibility age for New Zealand Superannuation. This policy is the most direct form of addressing the increasing fiscal cost of superannuation caused by increasing longevity. While it can be argued that there is no requirement to raise the age of eligibility provided the increasing cost can be borne, not doing so leaves the future cost of NZS more exposed to future unexpected increases in longevity than if a schedule of future increases in eligibility age were planned.

Estimates of future fiscal cost of NZS made by Treasury's Long-term Fiscal Model apparently yield politically acceptable results. However, the risk is that future mortality is better than assumed in the single scenario used: costs will in fact be greater than expected. A planned rise in eligibility age would be some insurance against this outcome which is likely according to the examination in this thesis of the plausibility of suggested future mortality trends. In fact, the increased cost from the likely outcome would be covered by an increase in eligibility age to 67 , even on a slower timetable than other countries. Thus longevity risk in New Zealand is also present as a result of failing to use a plausible range of mortality projections.

That New Zealand's public debate on eligibility age is relatively late and limited in nature is a further contributor to longevity risk. Where individual New Zealanders make unrealistically low assumptions for retirement age or lifespan, policy makers can help by providing signposts for both parameters. 
Eligibility age for NZS is a strong signal for intended retirement age, and the greater risk for New Zealanders in financial planning for retirement is from a low intended retirement age rather than longevity risk from underestimating likely age at death.

A wider debate about raising eligibility age would offer the opportunity for introducing the best available evidence on likely future lifespans into public discourse, improving from the flawed and underestimated data points most commonly referenced. This means referencing modal age at death, from period or cohort tables, or projected cohort life expectancies at the relevant age at least on the Medium Mortality basis, instead of the more common period measures. The potential for further lifespan improvement can be communicated by additionally referencing indicators from the Low Mortality scenario. However, the definition of the measure is more important for getting close to a likely age at death measure than the choice of mortality scenario.

This research was not directed at answering the question of whether New Zealand should have a higher eligibility age for its public pension. Other issues would also have to be addressed, such as preferences and equity considerations for spending on providing additional leisure years for older people compared to other potential demands. However, these findings provide evidence for a longevity rationale for raising eligibility age; suggest a potentially more compelling presentation of the policy than so far used in New Zealand, based on the individual perspective; and demonstrate the additional benefit of the policy from the mitigation of longevity risk. 


\section{Chapter 10: Conclusions and implications}

Longevity risk - the risk that future lifespans turn out to have been underestimated - is known as a potential threat to individual retirement planning and policy making. Demographers and actuaries have analysed past mortality trends and theorised about future mortality patterns, understanding the inherent uncertainty in forecasting future mortality and the recent history of underestimating population average lifespan. This thesis has probed some unanswered questions about why longevity risk should exist when a great deal of intellectual activity is taken up with projecting future mortality, and, using New Zealand as an example population, specifically examined the extent to which underestimation of likely future lifespans occurs and the consequences for longevity risk.

The starting hypothesis was that there are structural reasons in the way lifespans are measured and communicated that lead to longevity risk in both individual retirement planning and policy making settings. This chapter describes the findings of this thesis that confirm this hypothesis and illustrate the New Zealand case. It shows that, first, longevity risk is inherent and likely widespread when mortality is improving; second, that the relatively positive mortality outlook for New Zealand means future lifespans are likely to turn out better than the main official estimate; third, that New Zealanders underestimate likely lifespans; and fourth, that lifespan indicators used in public discourse may hold the key to reducing longevity risk in both individual planning and policy domains. A final section summarises the contribution to theory this thesis has made. 


\subsection{Longevity risk inherent and widespread}

Longevity risk is known to be a threat in public pension policy, even after reforms to tackle ageing such as raising the age of eligibility of public pensions, and in individual retirement planning. In neither domain has the extent, causes and consequences of longevity risk been well investigated. Limited empirical evidence from survey data in the United Kingdom and United States of America suggests lifespans are more often underestimated that otherwise.

Longevity risk is more likely to exist now than in the 1950s and 1960s. Then, mortality rates were fairly stable in most developed countries or even slightly worsening. Now, mortality rates are improving and average lifespans are lengthening. Moreover, it is well known amongst demographers and actuaries that the pattern of mortality trends is changing: there has been unexpected rapid improvement in mortality rates at older ages in developed countries since the 1980s. The consequences of these patterns for future trends are a source of active debate, but the consensus among demographers and actuaries is for further gains in average lifespan. All mortality forecasting is essentially extrapolation of past trends. When mortality is improving, overreliance on extrapolation without due consideration of potential changes in trend is likely to lead to underestimation of future lifespans; and has done so in developed countries since the 1980s.

It is known in demographic and actuarial theory and practice that period life expectancy, at any age but especially at birth, is a flawed measure of actual lifespans, being better suited as a summary indicator of population average 
mortality experience at a point in time. Cohort life expectancy allows for changing mortality rates during the lifecourse of a defined birth cohort and is therefore closer to the intuitive meaning of what lifespan an individual should expect. Despite this, cohort measures are rarely used, whereas period life expectancy at birth is used in many settings, including in official mortality projections. Existing literature does not explore the consequences of the almost universal use of period measures in public discourse. However, one consequence is demonstrated here: the misuse of period life expectancy, especially life expectancy at birth, to set individual lifespan expectations in informal and formal settings in New Zealand and elsewhere. Period measures will be lower than the more appropriate cohort measures of lifespan when mortality is improving, and will increase more slowly. Therefore, such misuse contributes to a structural reason for lifespans to be underestimated. The reason for such misuse is conjectured here to stem from the original naming of 'expectation of life' in a setting which no longer applies. The more appropriate cohort measures are available in New Zealand, the UK and other countries with an actuarial input to forecasting but are widely referenced for policy making and in public discourse only in the UK.

Furthermore, period life expectancy is increasingly problematic as a measure of likely lifespans in countries where its value is high, over 80 years, and mortality is shifting and compressing. As the shape of the distribution of ages at death changes, life expectancy at birth, the average of the distribution, becomes less useful as a single indicator of change. In these circumstances, modal age at death is sensitive to changes in mortality at the oldest ages, and will be higher than period life expectancy at birth, even within the same period life table. In countries including New Zealand which are advanced on the 
path to mortality compression with mortality change taking place at the oldest ages, the mode is an increasingly useful measure of the central tendency of population age at death.

Because available data suggests a tendency to underestimate future lifespans by both individuals (in subjective longevity expectations) and policy makers (in the mortality projections provided to them by official agencies), there is likely to be widespread longevity risk in individual retirement planning and policy making settings. Furthermore, there appears to be a culprit in the almost ubiquitous measure of lifespan proven as flawed and of diminishing usefulness. However, to confirm or otherwise the hypothesis that this contributes to a structural reason for longevity risk, further exploration was made into the lifespan assumptions prevalent in policy making and retirement planning, taking New Zealand as a case example.

\subsection{Future mortality outlook in New Zealand positive}

Longevity risk is exacerbated if public information on future mortality or lifespan is conservative, that is, underestimates lifespans. Public information on future lifespans is sourced in most countries from official statistical agencies. These agencies use similar mathematical techniques to extrapolate assumed future trends in mortality rates from past trends.

However, new doubts about relying purely on extrapolation are beginning to be expressed. The fast pace of mortality improvement at older ages and the changing shape of the distribution of ages at death produces a situation never seen before which may presage a break from the hitherto smooth improvement in average population lifespan. Moreover, demographic theory 
attempting to explain the variance of lifespan within populations is only now emerging. This theory also sows doubt on whether simple extrapolation of average mortality measures from past to future can still be a valid basis for mortality projections in high life expectancy countries like New Zealand.

Uncertainty about future lifespans is therefore increasing. The uncertainty is about the pace of future mortality improvement; the question is how to estimate it. Understanding past mortality trends has long been considered a necessary activity before attempting to forecast future population mortality, although the extent to which such analysis influences official projections of future mortality is hard to appreciate from published reports. There is no one formula to which one can refer to predict future age at death for an individual or for the average of a population. There are too many possible contributory factors - environmental, genetic, behavioural and medical - with an overlay of randomness. It is not possible to quantify the potential future population mortality risk from single causes. Only smoking with its single main cause of death has a reliable past evidence base of causation. Therefore, instead of accumulating evidence on past causes of death as a source of insight to supplement pure extrapolation, international comparisons of past average population mortality trends are recommended. However, such analysis is rarely carried out, and any reported on in support of official mortality projections appears cursory.

This thesis has carried out detailed analysis of New Zealand's mortality compared to that of its British settler country peers. Mortality in New Zealand ranks in the middle of the groups of British settler countries selected for this analysis, the others being Australia, Canada, the United Kingdom (UK) and 
United States of America (US). This is the case looking at both the level of mortality rates and period life expectancy at birth used here in its appropriate form as a summary measure of all-ages population mortality at points in time. There is slightly better mortality in Canada and still better mortality in Australia. There is slightly worse mortality in the UK and still worse in the US. In all these countries mortality rates are lower and life expectancy is higher for females than males. Relative to the other countries, New Zealand's mortality appears comparatively better for females than males.

Although the 1960s were a decade of slow or no improvement in mortality, there has since been constant improvement in all countries. The improvement in all-age mortality rates over the 45 years, standardised to the New Zealand population age profile by gender was 1.3 per cent for both males and females in the US; 1.6 per cent in the UK; and 1.7 per cent for $\mathrm{New}$ Zealand. The rates for Canada were 1.8 per cent for females and 2.0 per cent for males; from Australia 2.1 per cent and 2.2 per cent respectively. New Zealand's mortality improved over the evaluation period against Canada, the UK and US at ages over 40, but worsened at younger ages especially for males. The improvement in New Zealand's mortality accelerated for females in 1980s and males in 1990s. In the last decade the rate of improvement in mortality rates in New Zealand was the highest of all countries for female and second only to the outperformance of Australia for males. Thus the very fast improvement in mortality levels in Australia has been striking over the fortyfive year period, and New Zealand's recent experience has come very close. 
Mortality analysis in actuarial literature has focused recently on the existence of a 'golden cohort' of people born in the UK before and during the Second World War who have had higher annual rates of improvement in mortality rates than preceding or following cohorts at each age through their lifetime. The analysis in this thesis revealed that all the countries selected here have such a golden cohort with birth years covering the period 1922-1941. The UK is unusual in that its golden cohort is most clearly defined by the extent of the difference to mortality improvement levels in other cohorts, but also because it does not have a putative younger golden cohort visible in all the other countries centred on birth years 1952-1976. Relatively, New Zealand has had poor mortality levels at ages 1 to 20 although high rates of improvement at ages 15-25 in the last decade. Over the longer term of this analysis New Zealand has had particularly strong mortality improvement at ages over 40 and unusually high rates of mortality improvement at ages over 75 . This bias towards good mortality at older ages is further indicated by New Zealand having had the highest increase in modal age at death among these countries with the most recent data showing New Zealand with the highest modal age at death for females.

The official projections of New Zealand's future life expectancy are similar to those in the other countries selected for this analysis in that they are based on an extrapolation of past trends and therefore result in projected continued lengthening of average population lifespan. The difference between projections of different countries is the assumed trajectory of future mortality improvement. The analysis of the projections made for this thesis revealed generally simple and somewhat arbitrary assumptions for this trajectory. All the projections are 'what-if' scenarios essentially assuming a simplified 
continuation of the historic average mortality trend, but with different implicit or explicit choices made about the shape of the trajectory. A comparison of the outcomes of the central projections, as measured by future life expectancy measures, shows that Australia retains the top ranking and the US the lowest. There would be no reason to suggest otherwise, given the relative positions each country's mortality sustained over the forty-five years of historic analysis. However, the mortality of Canada is projected to worsen relative to its peers, the UK's to improve significantly, and New Zealand's and the UK's to diverge even though current and past life expectancy measures have been close. Existing mortality analyses have provided no explanation for why this should be so.

Instead, analysis here of the assumptions underlying New Zealand's mortality projections compared to those of its most relevant mortality role models have confirmed that New Zealand's appear relatively pessimistic. Underlying Australia's projections are more optimistic assumptions for future mortality than those made in New Zealand's because recent mortality improvement is given more weight than earlier, slower change. Following this approach for New Zealand would increase the rate of mortality improvement assumed. The UK's projections appear relatively optimistic because they allow for high mortality improvements in the short-term future - a path which based on recent experience New Zealand may be more likely to follow. By seeking consistency between the assumptions and outcomes of these relevant comparator projections, the Low Mortality variant of Statistics New Zealand's projections has a plausible narrative. 
Further analysis of aspects of New Zealand's mortality provides no evidence to alter this conclusion. In particular, demographic opinion tends to the view that obesity will not cause average mortality levels to increase in future. Increasing average body mass index in New Zealand and all comparator populations has coexisted with improving average mortality levels over recent decades. Medical treatments and prevention appear able to reduce mortality risk from obesity, and improvements in non-obesity related mortality continue. The rise in obesity prevalence appears to be levelling off. Against the potential for a surprising worsening mortality trend must be set the evidence for New Zealand's better fundamentals for long life than most countries can offer: cleaner air, lower population density and more benign climate. The evidence-based reasoning presented here, based on more detailed analysis of past and future projected mortality than has been presented elsewhere, points to future mortality in New Zealand being better than the main official projection and plausibly like the Low Mortality variant of Statistics New Zealand's projections.

Further testing of the robustness of this conclusion was carried out by applying demographic theories to draw conclusions from a comparison of mortality rates, life expectancy and mortality variance between subpopulations of the New Zealand total population over the forty-five year period. Specifically, the characteristics in those parameters for the Māori subpopulation as compared to non-Māori and those of males compared to females appear as expected following these theories. Further, the changing mix of migrants to New Zealand is not likely to have a large unanticipated impact on average mortality trends. Thus, no evidence is available to suggest that mortality changes occurring within the New Zealand population are 
likely to disturb the extrapolation of total population mortality trends. However, data definition inconsistencies and fluidity in the ethnic concept for population and death registrations make it difficult to interpret trends and absolute values of mortality by ethnicity in New Zealand. Importantly, the reliance on period life tables and lack of reliable cohort life expectancies by ethnicity means that the available period life expectancies for Māori and nonMāori understate actual lifespans and will likely overstate the gap between realistic average lifespans of these ethnic groups.

Consideration of demographic theories of mortality or age at death variance confirmed that variation will remain: it is an inherent characteristic of mortality. Although epidemiologic studies have focused on the socioeconomic drivers of mortality variation, these do not provide a complete explanation of cause or consequence. On one measure of age at death variance which standardises for level of life expectancy, New Zealand appears to have somewhat low variance for men but high for women, within the groups of comparator British settler countries. A reduction in mortality at young ages would reduce the level of variance in age at death in New Zealand although it would not improve average all-age measures such as period life expectancy by any significant amount. Given New Zealand's poor relative levels of mortality rates at ages 1-20, policy actions to reduce deaths at these ages, such as tackling road traffic and other accidents are justified. However, demographic theory suggests that age at death variance may increase in future in countries well advanced on the path to mortality compression because of increasing heterogeneity of frailty as more people survive to the oldest ages. This would be true in the case of New Zealand, especially given its relatively good mortality at older ages. 
Thus at this current phase of emerging theory and limited empirical data, and assuming efforts are made to tackle mortality differences where causes can be isolated, the picture of mortality variance appears to lend support for relative and absolute optimism about future population mortality in $\mathrm{New}$ Zealand, and the Low Mortality variant projection to be more likely than the Medium Mortality estimate. The latter is more commonly used, and where it is referenced, future lifespans are likely to be underestimated and longevity risk therefore exists.

\subsection{New Zealanders underestimate likely lifespans}

Subjective longevity expectations, that is, how long people think they are likely to live, should be an important element of individuals' own retirement planning. Further, the subjective view of policy makers on the outlook for longevity may colour their interpretation of demographic data and policy decisions made using it. However, there has been no data on longevity expectations in New Zealand, and few large sample surveys on the subject internationally. The mechanisms by which people set their expectations are not understood. Differences in expectations, the accuracy of expectations, or what factors cause people to have those expectations have not been well explored. This thesis addressed these research gaps with a review of existing literature to extract findings relevant to a national population and a detailed survey of the lifespan expectations of adult new Zealanders.

Studies based on surveys asking for individuals' expectations for their own longevity are found in the literature of actuarial science, anthropology, demographics, economics, health studies, policy, psychology and statistics. Of 
the reports of findings based on surveys of "subjective life expectancy" or "subjectivity probability of survival" only six were from original large datasets of longevity expectations using national adult population samples. These either originated in the UK or US. Only one survey, from Great Britain, covered all adult ages, had no other limitation of scope and quantified the gap between given subjective lifespan and life expectancy as measured by a population life table for each respondent by age and gender. This single study (O'Brien et al. 2005) was not in a peer-reviewed journal, but found that on average subjective lifespan as given was slightly below contemporary period life tables, but more significantly below the population cohort measures which allowed for the then best estimate of future improvement in mortality, at around four years on average for males and six years for females. The authors concluded that people had a sense of a prevailing wisdom based on current period measures of life expectancy but did not appreciate that the future was likely to bring improving mortality. The accumulated evidence from the other studies pointed to people holding subjective longevity expectations congruent to contemporary period tables and so exhibiting a prevailing tendency to underestimate potential longevity more than overestimate. Longevity risk therefore existed in all these samples.

The survey of subjective lifespan expectations carried out in New Zealand confirmed the hypothesis that a similar pattern of underestimation of lifespans exists. Subjective lifespan (SLS) was captured by asking "'What age do you think you will live to?" to a sample representative of the New Zealand adult population $(\mathrm{n}=850)$. Average underestimation of lifespans was around four years compared to Statistics New Zealand's Medium Mortality cohort table and over five years for males and over seven years for females compared to 
the cohort measures from the Low Mortality projection which this thesis proposes as the most plausible variant. The proportions of adult New Zealanders underestimating their SLS based on the period table contemporary to the survey was 45 per cent, with 25 per cent giving SLS congruent to the table and 30 per cent overestimating. Using the Medium Mortality projection for future mortality improvement, those figures are respectively, 56, 24 and 20 per cent. Compared to the preferred Low Mortality projection, 65 per cent of adult New Zealanders underestimated their lifespan, 20 per cent gave congruent SLS and 15 per cent overestimated. Thus, when asked for their likely age at death, more adult New Zealanders give answers that underestimate what is considered likely in population age- and genderspecific population projections of future lifespans than give higher answers or answers close to the projections. The maximum underestimation of SLS was 30 years on the Low Mortality cohort table. Underestimation of lifespans in New Zealand is therefore widespread and can be significant.

As appeared to be the case in previous surveys, it is likely that New Zealanders take cues for their likely lifespan from the contemporary pattern of deaths in the population. The female distribution of SLS had a single, strong peak at age 85. The male distribution was flat from 75 to 85 . Both features are similar in the distribution of age of deaths contemporary to the survey, although the male SLS tended towards age 75 more than the actual distribution. Within six months before this survey, life expectancy at birth from the most recent complete period life table (2005-7) had been publicised in New Zealand, erroneously, as what should be expected as a lifespan for those recently born: 82 years for females and 78 years for males. The evidence from this New Zealand survey is consistent with the hypothesis that individuals 
form lifespan expectations without appreciating likely future mortality improvement; and that indicators in the public domain exert some influence.

Subgroups of male New Zealanders appeared both over-pessimistic and over-optimistic about SLS: higher proportions of men than women gave SLS at both low and high extremes. Both subgroups were associated with low financial knowledge. Individuals aged under age 30 were more likely to underestimate than older people; the effect of unanticipated mortality improvement accumulates for longer for younger people. Other than age and gender, no other demographic mortality risk factors appear salient predictors of underestimation.

By asking why respondents chose their SLS, new insights emerged on how longevity expectations are formed. Great diversity in reasoning was apparent, so no one model for how the population thinks about SLS is appropriate. Three quarters of the New Zealand adult population reason their SLS on the basis of external, given factors or none; only one quarter reason on the basis of personal risk factors which they could influence. This appears to underplay the actual likely impact on mortality risk of lifestyle and behaviour. Within the larger group, few believe their SLS is 'normal', with most either putting faith in their genes or in a variation of lifespan being 'out of their hands'. Therefore, information on likely future population average SLS may simply not be believed by many without some other narrative or rationale.

The risk of underestimating lifespan appeared to be strongly associated with the reason behind choosing SLS. Those who do not have a reason, or who give a reason on the basis of their SLS being normal or who cite their own 
bad mortality risk factors are more likely to give a low or very low SLS. All could be at risk of underestimating lifespan; the latter group are still are at risk if their perception of bad mortality risk is incorrect. Those who cite good risk factors or genes or family history of lifespan are more likely to choose a high SLS but may still underestimate. The extent to which perceived 'good' risk factors will extend longevity may not be fully appreciated. Those basing their SLS on parents' or grandparents' lifespan may not allow sufficiently for mortality improvement over time. Those choosing the lowest SLS may turn out to have actual lifespan in the lower range, but still have longevity risk from living longer than they expect, and may not have money saved for retirement because this group is also associated with having low financial knowledge or low net worth. Those who choose the highest SLS are also more likely to have low financial knowledge, suggesting that they may be a group of confident optimists rather than accurate predictors or planners. Whether or not they turn out to live as long as they expect, their longevity risk may arise from poor financial planning for retirement.

The survey revealed how infrequently New Zealanders incorporate lifespan expectations into retirement planning. Financial planning for retirement is given some thought by just over half the adult population, whereas just over two thirds have thought about the age to which they will live. However, only around one quarter suggested SLS as a factor to be considered in retirement planning. Therefore, in thinking about ways to mitigate longevity risk within retirement planning, it might be considered a first priority to encourage the activity of retirement planning, and then how to offer realistic assumptions for the necessary planning parameters which 
should include the length of retirement: a function of retirement age and age at death.

New Zealanders' stated expectations for likely retirement age are, for the majority, either age 60 or age 65 . The duration of retirement people expect can be calculated from subtracting stated expected retirement age from SLS, but because of the lack of thought about retirement planning, the resulting value can only be called "implied" length of retirement. A narrow range of expected retirement ages co-exists with a wide range of implied length of retirement. Implied retirement duration expected by New Zealanders fell into three equally-sized groups: up to and including 10 years, 11 to 20 years and 21 to 40 years. Longevity risk can exist whatever the implied length of retirement. Some people expect too short a retirement than they are likely to experience; explicitly or implicitly, including some cases of not having a retirement plan. The risk in expecting a short retirement is not being able to find or be able to do the work they expect to have at older ages; or in finding that their retirement income does not last if they do live longer than they expect. Those expecting a long retirement may not appreciate the large amount of savings needed to provide an income for that long, and they may be unrealistic about either or both of their assumed low retirement age and SLS. Thus for many New Zealanders expectations for retirement age and age at death are either not used in retirement planning or are used unrealistically. Longevity risk the risk of 'dying too late' - is compounded by the risk of 'retiring too early'. 


\subsection{Lifespan indicators key to reducing longevity risk}

Public pension policy exerts a strong influence on retirement planning as it sets eligibility age for the public pension, which appears to be a very strong driver for intended retirement age. Further, subjective lifespan expectations appear to be made following cues from information on lifespans referenced in public discourse, and these indicators come from the same official statistical agency which provides policy makers with the mortality data for their economic modelling. The most commonly used lifespan measures have been shown here to be underestimates of potential lifespans and the most commonly used scenarios of population mortality projections shown to be conservative in the case of New Zealand. The sources of longevity risk in New Zealand have therefore been explored within policy for eligibility age for New Zealand Superannuation; within the projection data used for estimates of future likely lifespans in policy making and within indicators used in public discourse on likely future lifespans.

\section{Not increasing eligibility age increases longevity risk}

There are currently (late 2011) no plans to increase eligibility age for New Zealand Superannuation (NZS); indeed such a change has been ruled out by the current government. However, an increase in eligibility age would directly address the increasing fiscal cost of superannuation caused by increasing longevity. Of the British settler countries used as comparators to New Zealand in this thesis, the US has started to raise eligibility age for its main public pension from 65 to 67; Australia plans to do so and the UK plans to raise the age still further to 68 . Longevity risk within public pension policy in New Zealand is therefore greater than that in these countries, and especially 
compared to the UK and US as New Zealand's mortality is lower and improving faster.

The given reason for not increasing New Zealand's eligibility age is that estimates of the future fiscal cost of NZS yield acceptable results. However, these costs, estimated using Treasury's Long-Term Fiscal Model, refer to a single scenario for future mortality: Statistics New Zealand's Medium Mortality. According to the results of this thesis, a more plausible outlook for future mortality is more optimistic. It is therefore likely that the fiscal costs of NZS will be higher than modelled. Longevity risk in policy planning is introduced from using a conservative mortality scenario. A planned rise in eligibility age would be some insurance against this longevity risk. In fact, an increase in eligibility age to 67 , even on a slower timetable than other countries are planning, has been estimated to cover the increase in cost from the lower mortality envisaged in the more plausible scenario, compared to the single scenario used. Thus there is longevity risk in New Zealand Superannuation policy, greater than that in other countries, from failure to plan to increase eligibility age and compounded by failing to use a plausible range of mortality projections.

\section{Conservatism in future mortality projections}

The reliance on a single central estimate for future mortality as input to fiscal cost modelling for policy purposes is not confined to New Zealand. It is common practice in most countries. Official projections of future mortality appear technically detailed and are the result of a great deal of modelling activity which is difficult for the non expert user to understand let alone challenge. The need to understand the detailed analysis is belied by the 
inevitably rather arbitrary choice of assumptions for future projections; but this may not be obvious to a user. The nuanced perspective of the demographer distinguishing between "forecast" and "projection" may not be appreciated. The non-expert user may be misled into thinking that the complexities of forecasting have been considered by the experts, and that their central estimate represents a single best estimate. Generally "Low" and "High" variants are chosen to represent an arbitrary equal difference in mortality improvement rates on either side of the central estimate, so shed limited light on the potential range of outcomes. The handover of official evidence on future lifespans from official statistical agency to policy maker therefore tends to result in the use of a single projection based on unquestioned assumptions. There is generally no detailed analysis made available which could help a nonexpert user to decide which future scenarios for mortality should be tested for fiscal consequences. Fuller data, including less conservative projections which may actually represent more plausible future mortality paths, is lost.

Further, in New Zealand and other countries, the official agency appears to prefer not to present the results of mortality projections in terms of future cohort life expectancies. This is a conservative approach, as if too much reliance should not be put on the underlying assumptions of future mortality change in the cohort measures; even though these assumptions are identical to those underlying the official projections. This may be a consequence of the statistical agency's primary role in making projections being to estimate the size of the national population. Mortality is only one element alongside fertility and migration in the population projections. In the case of New Zealand, the statistical agency specifically warns against using its mortality assumptions "as a precise measure of mortality" and states that the "objective 
of population projections is not to specifically measure or project the life expectancy of the population" (Statistics New Zealand 2009a). Yet naturally, there is great interest in what are assumed to be measures of lifespan; they are referenced widely and often misunderstood.

This preference not to publish cohort measures leads to a reliance on the available period measures. These, by definition, assume there will be no change in mortality rates in future. In the current era of improving mortality this assumption is not just conservative but unlikely. The persistent rate of mortality improvement in the countries selected for this analysis confirms that past period life expectancies cannot be relevant historic lifespan indicators, and the shared assumptions of continued mortality improvement mean that projected period life expectancies cannot be relevant future lifespan measures. Longevity risk is introduced by setting period life expectancy measures, which underestimate lifespans, as the most usual form of public information about lifespans.

\section{Indicators in public discourse on future lifespans underestimate}

Where individual New Zealanders make unrealistically low assumptions for retirement age and lifespan, policy makers can help by providing signposts for both parameters. Again, eligibility age for New Zealand Superannuation is critical. It is a strong signal for intended retirement age, and the greater risk for New Zealanders in financial planning for retirement is from a low intended retirement age rather than longevity risk from underestimating likely age at death. 
Further, as has happened in other countries, a wider debate than has been held so far in New Zealand about raising eligibility age would offer the opportunity for introducing the best available evidence on likely future lifespans into public discourse, improving from the flawed and underestimated data points most commonly referenced. This means referencing projected cohort life expectancies at the relevant age at least on the Medium Mortality basis, instead of the more common period measures. The potential for further lifespan improvement can be communicated by additionally referencing indicators on the Low Mortality scenario or indicators of the extreme ends of the distribution of current ages at death.

The contribution to underestimation of lifespans from using a flawed measure - period as opposed to cohort - is greater than the contribution from using a more plausible assumption for future mortality than the almost universally used medium estimate. Illustrating with Statistics New Zealand data points that might be used in retirement planning by an individual born in 1960, a lifespan thought to be appropriate by reference to the latest period life expectancy at birth would be an underestimate of eight (female) or ten (male) years compared to the year of birth-specific cohort measure on the plausible (Low Mortality) scenario. Assuming survival to the age of retirement intention (that is using period life expectancy at retirement age) improves on assuming the period life expectancy at birth measure by adding four years for females and five for males. The remainder of the shortfall is made up by using the year of birth-specific cohort measure on the plausible scenario, but all but one of these years comes from the change from period to cohort measure, rather than from the choice of scenario of future mortality improvement. 
New Zealanders' subjective longevity expectations are chosen for a variety of reasons, and public information on potential future lifespans may not be believed or acted on by everyone. However, the definition of the measure is important for getting close to a likely age at death measure. In an era of improving mortality, individuals may not appreciate that their own likely lifespan is longer than their parents, grandparents or what they see around them at the current time. It seems an obvious responsibility of demographers, actuaries and official users of the data to ensure the indicators in public discourse are theoretically robust and plausible, and to carry the message that longevity is improving.

In Australia, the official actuarial projections of future mortality (AGA 2009) are based on two variants rather than three, which is likely to be a better prompt for the user to make an informed decision which one to use rather take the easy decision to use the central estimate out of three. In the UK, the presentation of future mortality projections is made in terms of cohort life expectancies by the official statistical agency and the government department responsible for pension policy (DWP 2011; ONS 2009). Modal age at death is rarely if ever used as an indicator of population lifespan yet it is simple, easily available and less likely than period measures to underestimate. Each of these actions would go some way to mitigate the systemic problem of underestimation caused either by over-reliance on life expectancy at birth as a lifespan indicator or over reliance on a conservative central projection of future mortality. Each is possible in New Zealand. 
Mortality is inherently uncertain. An individual cannot predict the age of his or her own death, and a demographer or actuary cannot give a policy maker an accurate prediction of average future lifespans. Yet the indicators of potential lifespans in public discourse are nearly always flawed. The result of these flaws acts in one direction: to communicate low estimates compared to what is likely. The innate conservatism of projections of future lifespans by demographers, and the failure of policy makers to question the underlying assumptions of the main projection offered, leads to further underestimation. Longevity risk is the result, both in individual retirement planning and policy domains. Longevity risk exists in New Zealand, more so than in other countries which are planning to increase eligibility age of the public pension. Increasing the eligibility age of New Zealand Superannuation would itself help to mitigate longevity risk. Whether or not the age is raised a more active public debate on the issue would give the opportunity for technically correct indicators of future increasing lifespans to be set into public consciousness.

\subsection{Contribution to theory}

This thesis has explored the existence, causation and remedies for longevity risk in two under-explored domains: individual retirement planning and public pension policy. In doing so, it has taken a new theoretical perspective on measures of lifespan; extended existing demographic and actuarial theories to undertake original research on past mortality and lifespan prospects in New Zealand; built on an existing body of research on subjective lifespan expectations in a new setting, and accumulated theory and empirical findings to diagnose longevity risk and suggest remedies applicable in the general case as well as in New Zealand. 


\section{New theoretical perspective on measures of lifespan}

Brief acknowledgement of the shortcomings of period life expectancy to represent potential lifespan or to indicate population mortality trends in high life expectancy countries can be found within existing literature. Supplementary measures such as modal age of death or cohort measures are known as theoretically superior. Modal age at death and other life table based measures have been subject to limited empirical analysis but are not used practically. There have been applications of cohort life expectancy, to some extent in New Zealand and especially so in the UK.

In this thesis such commentary on the theoretical shortcomings of period life expectancy measures, especially period life expectancy at birth, has been brought together and developed to confirm the critical contribution these flaws make towards longevity risk. It has been demonstrated that period measures of life expectancy are used widely for a purpose they are increasingly not suited to, especially in high life expectancy countries like New Zealand. This conclusion has additionally drawn on empirical evidence of the flawed use of period measures, the well-known demographic theory of mortality compression and emerging demographic theories of mortality variance. It has led to a conclusion that longevity risk will be inherent in high life expectancy countries where period measures of life expectancy are the main descriptor of population lifespan.

Additional theoretical work in this thesis has brought together emerging demographic theory on variance in mortality or age at death. It has juxtaposed the purpose of mortality variance analysis within epidemiology and public health disciplines to identify the association of variance in health inequalities 
and socio-economic position with the proof from demographic theory that variation in mortality or lifespan is inherent. Further, it has drawn implications for the validity of extrapolative forecasting of mortality from demographic studies showing associations between mortality parameters: declining variation in total lifespan as life expectancy increases, increasing variance in remaining lifespan at older ages and differential gains in life expectancy depending on how advanced populations are on the path compression of mortality. This has suggested a new field of research which interprets epidemiological studies of health inequalities through the lens of demographic theories of mortality variance.

Further, a new application of these mortality variance theories has been tested in this thesis. If two subgroups within a subpopulation are at different stages on the path to compression of mortality, then their indicators of variation in lifespan, life expectancy and mortality rates will behave differently. This theory has been used here to show that the trends in mortality within the New Zealand population, by two subgroups of ethnicity and gender, have been broadly as expected. The implication offered is that extrapolation of population average mortality in this case is valid; it should not be disturbed by unexpected changes in subgroup mortality. Confirmation of this conclusion and other applications of this analysis would be useful topics for future research.

\section{Original research on mortality past and prospects in New Zealand}

Demographic and actuarial literature is rich in the theory of mortality trends and mortality forecasting or projection techniques. However, much of this is not obviously used in the projections of future mortality by official statistical 
agencies or others involved in the demography of national populations. For example, comparisons of mortality trends between countries is recommended as a source of insight when projecting future mortality of a nation, but detailed comparative analysis of past mortality tends to be carried out to prove a single theory rather than to draw conclusions for projecting forward. Comparisons of the assumptions and outcomes of future mortality projections of like countries hardly exist.

In this thesis, an original analytic framework was developed to fill these research gaps in the case of New Zealand. The framework was developed to test the consistency and plausibility of New Zealand's mortality projections against those of select countries, starting with a comparison of New Zealand's past total population mortality trends to those of other countries. A rich inventory of mortality data from the recent past of New Zealand and peer countries was therefore developed, as well as a first empirical comparison of the assumptions and results of mortality projections in the selected countries. This approach follows the philosophy of Hajnal (1955) to draw on analysis of past population data in order to project future demographic variables, and to do so with "more cogitation and less computation" than other approaches which favour data processing over thoughtful insight. This thesis provides an innovative example of Hajnal's approach applied to mortality.

This analysis has several features that are new to demographic or actuarial literature. It selected only the British settler countries of Australia, Canada, New Zealand, the UK and US for comparison because of the social and ancestral similarities of their populations. It focused on mortality since 1961 as recent trends are not well examined in literature yet may be more important 
than longer term trends as mortality compresses. The analysis did not follow the usual practice on relying on period life expectancy as a measure of lifespans or trend indicator of mortality but supplemented its use with analysis of the level of mortality rates, rates of improvement in mortality rates and other central measures of age at death. Actuarial techniques supplemented demographic analysis. The study of past rates of improvement in age-specific mortality rates (defined here as "QXI"), which has developed as a key theme in actuarial literature, were developed further here. Where available, mortality projections from the official actuarial agency were used for international comparison in preference to those from the demography-led statistical agency, because the more insightful cohort life expectancies are an actuarial-led measure.

This work therefore contributes a detailed empirical evidence base of New Zealand's recent past, current and possible future mortality profile and that of its peers. It is a mainly optimistic picture with New Zealand in the middle of this group of countries, with leading momentum in mortality improvement in the recent decade. This work shows New Zealand has particularly good mortality at older ages, but relatively poor mortality at ages under 20. Further research to correct this poor mortality is indicated. This thesis is the first known analysis of QXI that confirms the existence of the golden cohort in all five countries in the range of birth years 1922-1936 for males, extending a further five years for females, and the presence of a putative golden cohort in birth years 1952-1976 for males starting ten years later for females in all the countries except the UK. This finding is a particularly important contribution to the debate on the cause and implications of golden cohorts. It supplements Murphy's (2010) investigation of the existence of golden cohorts but provides 
an alternative conclusion: that cohort effects are as important than period effects, not less so. Further, the cohort mortality patterns provide a reason to doubt the relative optimism of the UK's mortality projections and the relative pessimism of New Zealand's.

Setting mortality projections in an international comparative context revealed the somewhat arbitrary choice of assumptions. Each set of projections is based on extrapolation of past mortality data with some judgement necessary in the choice of parameters. Extrapolation is acknowledged in theory and practice as essentially the only method available, and has strong support based on a rationale of hitherto smooth progression in population life expectancy. However, this thesis provides support instead for the doubts beginning to be expressed about the validity of pure extrapolation for high life expectancy countries. The relative positioning of future cohort life expectancies between Canada, the UK and New Zealand appears inconsistent with the rankings of recent mortality and improvement rates. The robustness of the evidence for the assumptions used for projection by official agencies, the range of variant projections presented, and the implications for the results of the projections being interpreted as more definitive best estimates than they are intended to be are all questionable. While problems with the robustness of projections are not unknown in the general case, this thesis develops a substantial evidence base to assess the mortality assumptions underlying the official projections of New Zealand. The central finding that the Low Mortality variant is more plausible than the Medium Mortality variant most commonly used is supported by detailed evidence and analysis and is the only known testing of any of the Statistics New Zealand projection variants. 


\section{Extends existing body of research on subjective lifespan expectations}

Studies based on surveys asking for individuals' expectations for their own longevity are found in a variety of literature, having been carried out for a range of purposes. For this thesis, the first study of an adult population's expected age at death in a retirement planning context representative of a full adult population was made. It is also the first study to probe reasons for responses made and the first study in New Zealand on the subject.

The findings of this survey helped to confirm the findings of previous studies that "subjective lifespan" (SLS) is generally an underestimate of likely lifespan. By a technically more advanced analysis of results than most other large studies, the distribution of the size of underestimation relative to established mortality scenarios strengthens the theory (hypothesised in earlier studies) that respondents fail to anticipate future improvement in mortality. The profile of underestimation was able to be explored by a large number of variables, but amongst mortality risk factors only age and gender emerged as salient predictors of lifespan underestimation. This suggests a systematic pattern of underestimation in populations - a notion unexplored in previous studies which have focused on associations between demographic factors and level of SLS imputing a rational logic to the choice of SLS. The results of this New Zealand study refute the notion that there can be a single model of SLS rational choice. No one model proposed to describe how individuals choose SLS fits the data in this study. Further, reasons for choosing SLS, which were ignored in previous studies, were identified as significant predictors for high or low SLS despite suggesting a lack of knowledge in respondents' minds on what likely lifespans might be. This leads directly to the theory that retirement planning is done without knowledge of likely length of retirement, and is 
subject to a population bias of underestimation because it relies on internalised contemporary evidence of ages at death. Further evidence was identified for the previously hypothesised failure to anticipate mortality improvement as around one-third of respondents cited their parents or grandparents' ages at death as the main reason for choosing age at death.

\section{Accumulates theory and empirical findings on longevity risk}

While existing literature acknowledges problems with extrapolative projections and even suggests solutions, there has been no empirical analysis of consequent longevity risk. This thesis filled this research gap using the case of New Zealand's superannuation policy and individual retirement planning.

To calculate the size of longevity risk requires the comparison of likely future lifespans and estimates of lifespans used in policy and individual retirement planning. This analysis of longevity risk required both a theoretical approach to determine what future lifespans are plausible and original empirical findings on subjective longevity expectations, with the identification of lifespan assumptions used in policy being a lesser task. The calculation of longevity risk in individual pre-retirement settings implicitly followed the widely used lifecycle savings hypothesis which underpins New Zealand's leading retirement planning tool. The calculation of longevity risk in the policy setting used existing calculations. Thus the contribution of this thesis in this area is not in the development of new tools to make calculations, but rather in the innovative approach that accumulated theory and evidence in order to explore longevity risk in practical circumstances, consistent with the Hajnal model. 
Furthermore, this thesis has developed theories and evidence on the cause of longevity risk. The larger part of underestimation of potential lifespans occurs when period measures of life expectancy are used but cohort measures are more relevant. Drawing on an illustration of potential assumed ages at death in an individual's retirement plan, half of the underestimation of age at death was shown to derive from misusing period life expectancy at birth instead of at retirement age and most of the remaining half from using period rather than cohort life expectancy measure. A smaller part of underestimation was indicated from using a conservative assumption for future mortality rather than a more plausible one. This new finding is important as it focuses the mitigation of longevity risk onto a structural issue - the use of lifespan indicators - rather than onto a partly subjective issue of which projection of future mortality is likely to be most plausible. In turn this strengthens the theory that using time- and context-specific indicators of lifespan in public discourse on superannuation policy would be an important remedy for longevity risk in both domains of policy and individual retirement planning.

Longevity risk has been found to be a potentially lesser risk in individual retirement planning than the risk of assuming too early a retirement age. The quantum of financial difficulty caused by 'retiring too soon' could be greater than that caused by 'living too long'. But the influence on intended retirement age identified from eligibility age of superannuation again strengthens the theory that the two domains considered here are inextricably linked, and that the signals sent by policy can cause or mitigate longevity risk for individuals. 
This research has provided the first confirmation that New Zealand is likely to be underestimating individual and population future longevity. The age of eligibility for New Zealand Superannuation is shown as a remedy for this longevity risk: as an indicator for retirement age and as a tool to communicate future lifespans and the consequences of mortality improvement. These results were not reached by questioning whether the age of eligibility should be raised or not, but they do contribute a robust argument for why an important policy decision should be publicly debated with correct evidence on potential individual lifespans. 


\section{References}

Abel, Guy J, Jakub Bijak and James Raymer. 2010. "A comparison of official population projections with Bayesian time series forecasts for England and Wales." Population Trends 141 (Autumn 2010).

ABS. 2008. "Population projections Australia 2006-2101". Australian Bureau of Statistics. Canberra.

ABS. 2010. "Year Book Australia, 2009-10". Australian Bureau of Statistics. Canberra.

AGA. 2009. "Australian Life Tables 2005-7". Australian Government Actuary. Canberra.

Andreev, K and J Vaupel. 2005. "Patterns of Mortality Improvement over Age and Time: Estimation, Presentation and Implications". Paper for conference: PAA 2005 Annual Meeting. Philadelphia, PA. 31 March - 2 April, 2005.

ANZ-Retirement Commission. 2009. "2009 Financial Knowledge Survey - Summary". www.financialliteracy.org.nz.

Arias, E. 2010a. "United States life tables by Hispanic origin". National Center for Health Statistics. Vital and health statistics Series 2 no. 152. Hyattsville, MD.

Arias, E. 2010b. "United States life tables, 2006". National Center for Health Statistics. National vital statistics reports vol. 58 no. 21. Hyattsville, MD.

Australian Government. 2009. "Pensions Overview: Secure and Sustainable Pensions". http://www.budget.gov.au/2009-

10/content/glossy/pension/html/pensions_overview_08.htm

http://www.budget.gov.au/2009-

10/content/glossy/pension/download/pensions_overview.pdf. Date accessed 25 January 2010.

Baker, David P., Juan Leon, Emily G. Smith Greenaway, John Collins and Marcela Movit. 2011. "The Education Effect on Population Health: A Reassessment."

Population and Development Review 37(2):307-332.

Banks, James, Carl Emmerson and Zoe Oldfield. 2004. "Not so brief lives: longevity expectations and wellbeing in retirement." In Seven Ages of Man and Woman, eds. Iain Stewart and Romesh Vaitilingam. Swindon: Economic and Social Research Council. 
Banks, James, Alastair Muriel and James P Smith. 2010. "Disease Prevalence, Disease Incidence, and Mortality in the United States and in England." Demography 47 (Supplement):S211-S231.

Barker, D J P. 2007. "The origins of the developmental origins theory." Journal of Internal Medicine 261:412-417.

Barr, Nicholas. 2000. "Reforming Pensions: Myths, Truths, and Policy Choices". International Monetary Fund Working Paper 00/139.

Bengtsson, Tommy. 2006. "Linear Increase in Life Expectancy: Past and Present." In Perspectives on Mortality Forecasting III. The Linear Rise in Life Expectancy: History and Prospects, ed. Tommy Bengtsson. Stockholm: Swedish Social Insurance Agency.

Benjamin, B. 1982. "The Span of Life." Journal of the Institute of Actuaries 109(3):319357.

Blakely, T, Tobias M, Atkinson J, Yeh L-C and Huang K. 2007. "Tracking Disparity: Trends in ethnic and socioeconomic inequalities in mortality, 1981-2004". Ministry of Health. Wellington.

Blakely, Tony, Martin Tobias and June Atkinson. 2008. "Inequalities in mortality during and after restructuring of the New Zealand economy: repeated cohort studies." BMJ 336:371-375.

Bloom, David E and David Canning. 2007. "Commentary: The Preston Curve 30 years on: still sparking fires." International Journal of Epidemiology 36:498-499.

Bongaarts, John. 2006. "How Long Will We Live?" Population and Development Review 32(4):605-628.

Booth, $\mathrm{H}$ and L Tickle. 2008. "Mortality modelling and forecasting: a review of methods." Annals of Actuarial Science I/II:3-43.

Booth, Heather. 2006. "Demographic forecasting: 1980 to 2005 in review."

International Journal of Forecasting 22:547-587.

Booth, Heather and Leonie Tickle. 2004. "Beyond three score years and ten: Prospects for longevity in Australia." People and Place 12(1):15-27.

Brouwer, Werner B F and N Job A van Exel. 2005. "Expectations regarding length and health related quality of life: Some empirical findings." Social Science \& Medicine 61:1083-1094.

Brown, J. R. 2001. "Private pensions, mortality risk, and the decision to annuitize." Journal of Public Economics 82(1):29-62. 
Brown, J. R. 2003. "Redistribution and Insurance: Mandatory Annuitizaton With Mortality Heterogeneity." Journal of Risk \& Insurance 70:17-41.

Bryant, John. 2003. "Can population projections be used for sensitivity tests on policy models?". The Treasury. New Zealand Treasury Working Paper 03/07. Wellington.

Burch, Thomas K. 2003. "Demography in a new key: A theory of population theory." Demographic Research 9:263-284.

Caldwell, J C. 1996. "Demography and Social Science." Population Studies 50:305-333.

Calvert, G N. 1946. "The Future Population of New Zealand". New Zealand Government, issued under the Authority of the Treasury. Wellington.

Canudas-Romo, Vladimir. 2010. "Three measures of longevity: Time trends and record values." Demography 47(2):299-312.

Carey, James R and Debra S Judge. 2001. "Life Span Extension in Humans is SelfReinforcing: A General Theory of Longevity." Population and Development Review 27(3):411-436.

Carey, James R. 2003. "Life Span: A Conceptual Overview." Population and Development Review 29:1-18.

Carnes, Bruce A and S Jay Olshansky. 2007. "A Realist View of Aging, Mortality, and Future Longevity." Population and Development Review 33(2):367-381.

Carter, Kristie N, Tony Blakely and Matthew Soeberg. 2010. "Trends in survival and life expectancy by ethnicity, income and smoking in New Zealand: 1980s to 2000s." The New Zealand Medical Journal 123(1320).

CFLRI, previously Retirement Commission. 2010. "Something will have to change to keep New Zealand Super in the future". Commission for Financial Literacy and Retirement Income. 7 December 2010. Wellington.

CFLRI, previously Retirement Commission. 2011. "Statement of Intent 2011/14". Commission for Financial Literacy and Retirement Income. Wellington.

Cheung, Siu Lan Karen and Jean-Marie Robine. 2007. "Increase in common longevity and the compression of mortality: The case of Japan." Population Studies 31(1):85-97.

Christensen, Kaare, Gabriele Doblhammer, Roland Rau and James W Vaupel. 2009.

"Ageing populations: the challenges ahead." Lancet 374:196-1208.

Christensen, Kaare, Thomas E Johnson and James W Vaupel. 2006. "The quest for genetic determinants of human longevity: challenges and insights." Nature Reviews. Genetics 7:436-448. 
Collinson, D, C Dey, G Hannah and L Stevenson. 2007. "Income inequality and child mortality in wealthy nations." Journal of Public Health 29(2):114-117.

Colmar Brunton. 2009. "2009 Financial Knowledge Survey". Prepared for the Retirement Commission, 17 June 2009. Wellington.

Crimmins, Eileen. 1993. "Demography: the Past 30 Years, the Present, and the Future." Demography 30(4):579-591.

Crimmins, Eileen, Jung Ki Kim and Sarinnapha Vasunilashorn. 2010a.

"Biodemography: New Approaches to Understanding Trends and Differences in Population Health and Mortality." Demography 47(Supplement):S41-S64.

Crimmins, Eileen M, Samuel H Preston and Barney Cohen eds. 2010b. Explaining Divergent Levels of Longevity in High-Income Countries. Washington DC: Panel on Understanding Divergent Trends in Longevity in High-Income Countries; National Research Council of the National Academies.

Cutler, David, Angus Deaton and Adriana Lleras-Muney. 2006. "The Determinants of Mortality." The Journal of Economic Perspectives 20(3):97-120.

Danaei, Goodarz, Mariel M. Finucane, John K. Lin, Gitanjali M. Singh, Christopher J. Paciorek, Melanie J. Cowan, Farshad Farzadfar, Gretchen A. Stevens, Stephen S. Lim, Leanne M. Riley and Majid Ezzati. 2011a. "National, regional, and global trends in systolic blood pressure since 1980: systematic analysis of health examination surveys and epidemiological studies with 786 country-years and 5.4 million participants." The Lancet 377(9765):568-577.

Danaei, Goodarz, Mariel M. Finucane, Yuan Lu, Gitanjali M. Singh, Melanie J. Cowan, Christopher J. Paciorek, John K. Lin, Farshad Farzadfar, Young-Ho Khang, Gretchen A. Stevens, Mayuree Rao, Mohammed K. Ali, Leanne M. Riley, Carolyn A. Robinson and Majid Ezzati. 2011b. "National, regional, and global trends in fasting plasma glucose and diabetes prevalence since 1980: systematic analysis of health examination surveys and epidemiological studies with 370 country-years and 2.7 million participants." The Lancet 378(9785):31-40.

de Grey, Aubrey D N J. 2006. "Extrapolaholics Anonymous: Why Demographers' Rejections of a Huge Rise in Cohort Life Expectancy in This Century are Overconfident." Annals of the New York Academy of Sciences 1067:83-93.

de Moivre, Abraham. 1725. "Annuities upon lives: or, the valuation of annuities upon any number of lives; as also, of reversions". Accessed through Eighteenth Century Collections Online.

Dowd, Kevin, David Blake and Andrew J G Cairns. 2010. "Facing up to uncertain life expectancy: The longevity fan charts." Demography 47(1):67-78. 
Dunnell, Karen. 2008. "Ageing and Mortality in the UK: National Statistician's Annual Article on the Population." Population Trends 134 (Winter 2008):6-23.

Dunstan, Kim and Nicholas Thomson. 2006. "Demographic Aspects of New Zealand's Ageing Population". Statistics New Zealand. Wellington.

DWP. 2010. "A sustainable State Pension:when the State Pension age will increase to 66. Cm 7956". Department for Work and Pensions. The Stationery Office. London.

DWP. 2011. "Cohort Estimates of Life Expectancy at Age 65". Department for Work and Pensions. London.

Ediev, Dalkahat M. 2011. "Life Expectancy in Developed Countries is Higher than Conventionally Estimated. Implications from Improved Measurement of Human Longevity." Population Ageing(4):5-32.

Engelman, Michal, Vladimir Canudas-Romo and Emily M. Agree. 2010. "The Implications of Increased Survivorship for Mortality Variation in Aging Populations." Population and Development Review 36(3):511-539.

Engen, Eric M, William G Gale and Cori Uccello. 2001. "Lifetime Earnings, Social Security Benefits, and the Adequacy of Retirement Wealth Accumulation Working Paper 2004-10". Center for Retirement Research at Boston College.

Epstein, David, Dolores Jimenez-Rubio, Peter C Smith and Marc Suhrcke. 2009. "Social Determinants of Health: An Economic Perspective." Health Economics 18:495502.

Eriksson, Johan G. 2005. "The fetal origins hypothesis - 10 years on." BMJ 330:10961097.

Falba, Tracy A and Susan H Busch. 2005. "Survival Expectations of the Obese: Is Excess Mortality Reflected in Perceptions?" Obesity Research 13(4):754-761.

Fallin, M Daniele and Amy Matteini. 2009. "Genetic Epidemiology in Aging Research." The Journals of Gerontology 64A(1):47-60.

Farzadfar, Farshad, Mariel M. Finucane, Goodarz Danaei, Pamela M. Pelizzari, Melanie J. Cowan, Christopher J. Paciorek, Gitanjali M. Singh, John K. Lin, Gretchen A. Stevens, Leanne M. Riley and Majid Ezzati. 2011. "National, regional, and global trends in serum total cholesterol since 1980: systematic analysis of health examination surveys and epidemiological studies with 321 country-years and 3.0 million participants." The Lancet 377(9765):578-586.

Finucane, Mariel M., Gretchen A. Stevens, Melanie J. Cowan, Goodarz Danaei, John K. Lin, Christopher J. Paciorek, Gitanjali M. Singh, Hialy R. Gutierrez, Yuan Lu, Adil N. Bahalim, Farshad Farzadfar, Leanne M. Riley and Majid Ezzati. 2011. "National, 
regional, and global trends in body-mass index since 1980: systematic analysis of health examination surveys and epidemiological studies with 960 country-years and 9.1 million participants." The Lancet 377(9765):557-567.

Fries, James F. 1980. "Aging, Natural Death, and the Compression of Morbidity." The New England Journal of Medicine July 17, 1980 303(3):130-135

Fries, James F. 1989. "The Compression of Morbidity: Near or Far?" The Milbank Quarterly 67(2):208-232.

Gallop, Adrian. 2008. "Mortality Projections in the United Kingdom". Paper for conference: Living to 100 and Beyond Symposium. Orlando, Fla.

Glei, Dana A. and Shiro Horiuchi. 2007. "The narrowing sex differential in life expectancy in high-income populations: Effects of differences in the age pattern of mortality." Population Studies: A Journal of Demography 61(2):141 - 159.

Goldring, Shayla, Nigel Henretty, Julie Mills, Kate Johnson and Steve Smallwood. 2011. "Mortality of the 'Golden Generation': What can the ONS Longitudinal Study tell us?" Population Trends 145 (Autumn 2011).

Goldstein, Joshua R. 2004. "Simpler Probabilistic Population Forecasts: Making Scenarios Work." International Statistical Review 72(1):93-106.

Gompertz, Benjamin. 1825. "On the Nature of the Function Expressive of the Law of Human Mortality, and on a New Mode of Determining the Value of Life Contingencies." Philosophical Transactions of the Royal Society of London 115:513583.

Gutterman, Sam. 2008. "Social Insurance Perspectives and Implications". Paper for conference: Living to 100 and Beyond. Orlando, Fla.

Haberman, Steven. 1995. "History of Actuarial Science." In The History of Actuarial Science, eds. Steven Haberman and Trevor A Sibbett: Pickering \& Chatto (Publishers) Ltd.

Hajnal, John. 1955. "The Prospects for Population Forecasts." Journal of the American Statistical Association 50(270):309-322.

Hamermesh, Daniel S. 1985. "Life Expectancy and Economic Behavior." The Quarterly Journal of Economics 100(2):389-408.

Harding, Seeromanie. 2003. "Mortality of Migrants from the Indian Subcontinent to England and Wales: Effect of Duration of Residence." Epidemiology 14(3):287-292.

Harper, Sarah and Kenneth Howse. 2008. "An Upper Limit to Human Longevity?" Journal of Population Ageing 1:99-106. 
Hering, Martin and Thomas R Klassen. 2011. "Is 70 the new 65? Raising the Eligibility Age in the Canada Pension Plan". Mowat Centre for Policy Innovation, School of Public Policy \& Governance, University of Toronto. Toronto.

Hinde, Andrew. 2007. Demographic Methods. London: Hodder Arnold.

Hobcraft, John, Jane Menken and Samuel Preston. 1982. "Age, Period, and Cohort Effects in Demography: A Review." Population Index 48(1):4-43.

Hoem, Jan. 2008. "The reporting of statistical significance in scientific journals." Demographic Research 18:437-442.

Holzmann, Robert and Joseph E Stiglitz eds. 2001. New Ideas about Old Age Security: Towards Sustainable Pension Systems in the 21st Century. Washington DC: The International Bank for Reconstruction and Development, The World Bank.

Hurd, Michael D and Kathleen McGarry. 1995. "Evaluation of the Subjective Probabilities of Survival in the Health and Retirement Study." The Journal of Human Resources 30 (Special Issue on the Health and Retirement Study : Data Quality and Early Results): S268-S292.

Hurd, Michael D and Kathleen McGarry. 2002. "The Predictive Validity of Subjective Probabilities of Survival." The Economic Journal 112 (October):966-985.

Hurd, Michael D, James P Smith and Julie M Zissimopoulos. 2003. "The Effects of Subjective Survival on Retirement and Social Security Claiming. Labor and Population Program Working Paper Series 03-11". RAND.

Huxley, Rachel R. and David R. Jacobs Jr. 2011. "Size still matters...but not in the way we once thought." The Lancet 377(9771):1051-1052.

Janssen, Fanny and Anton Kunst. 2007. "The choice among past trends as a basis for the prediction of future trends in old-age mortality." Population Studies 61(3):315-326.

Jatrana, Santosh and Tony Blakely. 2008. "Ethnic inequalities in mortality among the elderly in New Zealand." Australian and New Zealand Journal of Public Health 32(5):437-443.

Kannisto, Vaino. 1994. "Development of Oldest-old Mortality, 1950-1990: Odense Monographs on Population Aging, 1 ": Odense University Press.

Kannisto, Vaino. 2001. "Mode and Dispersion of the Length of Life." Population: An English Selection 13(1):159-171.

Keilman, Nico. 1997. "Ex-Post Errors in Official Population Forecasts in Industrialized Countries." Journal of Official Statistics 13(3):245-277. 
Keilman, Nico. 2003. "Types of Models for Projecting Mortality." In Perspectives on Mortality Forecasting 1. Current Practice, eds. Tommy Bengtsson and Nico Keilman. Stockholm: National Social Insurance Board.

Keilman, Nico. 2008. "European Demographic Forecasts Have Not Become More Accurate Over the Past 25 Years." Population and Development Review 34(1):137-153.

Keyfitz, Nathan. 1993. "Thirty Years of Demography and Demography." Demography 30(4):533-549.

Khwaja, Ahmed, Frank Sloan and Sukyung Chung. 2007. "The relationship between individual expectations and behaviors: Mortality expectations and smoking decisions." Journal of Risk and Uncertainty 35:179-201.

King, Gary and Samir Soneji. 2011. "The future of death in America." Demographic Research 25:1-38.

Kuh, D, R Hardy, M Hotopf, D A Lawlor, B Maughan, R Westendorp, R Cooper, S Black and G D Mishra. 2009. "A review of lifetime risk factors for mortality." British Actuarial Journal 15 Supplement, 1:17-64.

Lanchester, John. 2010. "The knives are out." In The Guardian Weekend, 2 October 2010. UK.

Lassetter, Jane H and Lynn C Callister. 2009. "The Impact of Migration on the Health of Voluntary Migrants in Western Societies: A Review of the Literature." Journal of Transcultural Nursing 20:93-104.

Lee, Ronald and Timothy Miller. 2001. "Evaluating the Performance of the Lee-Carter Method for Forecasting Mortality." Demography 38(4):537-549.

Leon, David A. 2011. "Trends in European life expectancy: a salutary view". International Journal of Epidemiology. First published online March 17, 2011. http://ije.oxfordjournals.org/content/early/2011/03/16/ije.dyr061.short. doi 10.1093/ije/dyr061.

Leslie, George. 1895. "The Rates of Mortality in New Zealand." New Zealand Journal of Insurance, Mining, and Finance September and November 1895.

Lessof, Elizabeth. 1949. "Mortality in New Zealand and England \& Wales." Population Studies 3(1):76-99.

Li, Nan and Ronald Lee. 2005. "Coherent Mortality Forecasts for a Group of Populations: An Extension of the Lee-Carter Method." Demography 42(3):575-594. 
Long, John F and Frederick W Hollmann. 2004. "Developing Official Stochastic Population Forecasts at the US Census Bureau." International Statistical Review 72(2):201-208.

Lutz, Wolfgang. 1995. "Scenario Analysis in Population Projections Working Paper WP-95-97". International Institute for Applied Systems Analysis (IIASA). Laxenburg Austria.

Lutz, Wolfgang. 2009. "Toward a Systematic, Argument-Based Approach to Defining Assumptions for Population Projections Interim Report IR-09-037". International Institute for Applied Systems Analysis (IIASA). Laxenburg, Austria.

Lutz, Wolfgang and Joshua R Goldstein. 2004. "Introduction: How to Deal with Uncertainty in Population Forecasting?" International Statistical Review 72(1):1-4.

Lynch, John, George Davey Smith, Sam Harper, Marianne Hillemeier, Nancy Ross, George A. Kaplan and Michael Wolfson. 2004. "Is Income Inequality a Determinant of Population Health? Part 1. A Systematic Review." The Milbank Quarterly 82(1):5-99.

Madrian, Brigitte. 2009. "Defaults and Savings Outcomes: Lessons, Research Gaps and Implication for Savings Policy". Paper for conference: Financial Literacy in Times of Turmoil and Retirement Insecurity. Washington DC. 20 March 2009.

Manski, Charles F. 2004. "Measuring Expectations." Econometrica 72(5):1329-1376.

Marmot, Michael. 2005. "Social determinants of health inequalities." The Lancet 365:1099-1104.

Mehta, Neil K. and Virginia W. Chang. 2011. "Secular Declines in the Association Between Obesity and Mortality in the United States." Population and Development Review 37(3):435-451.

Ministry of Health. 2008. "A Portrait of Health - Key results of the 2006/07 New Zealand Health Survey". Ministry of Health. Wellington.

Ministry of Health. 2010. "Mortality and Demographic Data 2007". Ministry of Health. Wellington.

Mirowsky, John. 1999. "Subjective life expectancy in the US: correspondence to actuarial estimates by age, sex and race." Social Science \& Medicine 49:967-979.

Mirowsky, John and Catherine E Ross. 2000. "Socioeconomic Status and Subjective Life Expectancy." Social Psychology Quarterly 63(2):133-151.

Murphy, M J. 1995. "The prospect of mortality: England and Wales and the United States of America, 1962-1989." British Actuarial Journal 1(II):331-350. 
Murphy, Michael. 2010. "Reexamining the Dominance of Birth Cohort Effects on Mortality." Population and Development Review 36(2):365-390.

New Zealand Herald. 15 November 2008. "Kiwis set to live longer in spite of health gloom, by Eugene Bingham".

http://www.nzherald.co.nz/nz/news/article.cfm?c_id=1\&objectid=10543134. doi

New Zealand Institute. 2010. "Life expectancy".

http://www.nzinstitute.org/index.php/nzahead/measures/life_expectancy/. Date accessed 12 July 2010.

New Zealand Labour Party. 2011. "Labour will give all Kiwis a secure retirement". http://www.ownourfuture.co.nz/savings. Date accessed 7 November 2011.

New Zealand Listener. 2010. "Many Happy Returns." In June 52010 issue, by Geraldine Johns

O'Brien, Chris, Paul Fenn and Stephen Diacon. 2005. "How long do people expect to live? Results and implications". Centre for Risk and Insurance Studies Research Report 2005-1. Nottingham University Business School.

O'Connell, Alison. 2011a. "How Long Do We Expect to Live? A Review of the Evidence." Journal of Population Ageing 4:185-201.

O'Connell, Alison. 2011b. "International forecasts of future longevity". Longevity Bulletin 01. Institute and Faculty of Actuaries. London.

O'Connell, Alison and Kim Dunstan. 2009. "Do cohort mortality trends emigrate? Insights on the UK's golden cohort from comparison with a British settler country." British Actuarial Journal 15, Supplement, 1:91-121.

OASDI Board of Trustees. 2010. "The 2010 Annual Report of the Board of Trustees of the Federal Old-Age and Survivors Insurance and Federal Disability Insurance Trust Funds". US Government Printing Office. Washington.

OCA. 2009. "Canada Pension Plan Mortality Study: Actuarial Study No. 7". Office of the Chief Actuary. Ottawa.

OECD. 2007. "Pension Reform: The Unfinished Agenda Policy Brief, September 2007". Organisation for Economic Co-operation and Development.

OECD. 2010. "OECD Health Data 2010 - Version: June 2010". http://www.oecd.org/document/30/0,3343,en_2649_34631_12968734_1_1_1_1,00.html. doi 
OECD. 2011a. "Pension reforms must deliver affordable and adequate benefits". Organisation for Economic Co-operation and Development. Media Release 17/03/2011.

OECD. 2011b. "Pensions at a Glance 2011: Retirement-income systems in OECD and G20 countries". OECD Publishing.

Oeppen, Jim and James W Vaupel. 2002. "Broken Limits to Life Expectancy." Science 296(10 May 2002):1029-1031.

Oliver, Adam and Don Nutbeam. 2003. "Addressing health inequalities in the United Kingdom: a case study." Journal of Public Health Medicine 25(4):281-287.

Olshansky, S Jay and Bruce A Carnes. 1997. "Ever Since Gompertz." Demography 34(1):1-15.

Olshansky, S Jay, Bruce A Carnes and Jacob Brody. 2002. "A Biodemographic Interpretation of Life Span." Population and Development Review 28(3):501-513.

Olshansky, S Jay, Dana P Goldman, Yuhui Zheng and John W Rowe. 2009. "Aging in America in the Twenty-first Century: Demographic Forecasts from the MacArthur Foundation Research Network on an Aging Society." The Milbank Quarterly 87(4):842-862.

Olshansky, S Jay, Douglas J Passaro, Ronald C Hershow and Jennifer Layden. 2005. "A Potential Decline in Life Expectancy in the United States in the 21st Century." The New England Journal of Medicine 352(11):1138-1145.

Olshansky, S. Jay, Bruce A. Carnes and Christine Cassel. 1990. "In Search of Methuselah: Estimating the Upper Limits to Human Longevity." Science 250(4981):634-640.

ONS. 2006. "National population projections 2004-based". Office for National Statistics.

ONS. 2009. "2008-based National Population Projections". Office for National Statistics.

Ouellette, Nadine and Robert Bourbeau. 2011. "Changes in the age-at-death distribution in four low mortality countries: A nonparametric approach." Demographic Research 25:595-628.

Peller, Sigismund. 1948. "Mortality, Past and Future." Population Studies 1(4):405-456. Pensions Commission. 2004. "Pensions: Challenges and Choices, The First Report of the Pensions Commission". The Stationery Office. London. 
Pensions Commission. 2005. "A New Pension Settlement for the Twenty-First Century, The Second Report of the Pensions Commission". The Stationery Office. London.

Perozek, Maria. 2008. "Using Subjective Expectations to Forecast Longevity: Do Survey Respondents Know Something We Don't Know?" Demography 45(1):95-113.

Phelan, Jo C., Bruce G. Link and Parisa Tehranifar. 2010. "Social Conditions as Fundamental Causes of Health Inequalities." Journal of Health and Social Behavior 51(1 suppl):S28-S40.

Phillips, Jock and Terry Hearn. 2008. Settlers: New Zealand immigrants from England, Ireland and Scotland 1800-1945: Auckland University Press.

Pool, Ian. 1982. "Is New Zealand a "Healthy Country"? The Centenary of Dr Alfred Newman's Affirmation "that it is the healthiest in the face of the Globe"." New Zealand Population Review 8(2):2-27.

Pool, Ian. 1985. "Mortality Trends and Differentials." In Country Monograph Series No 12: Population of New Zealand Volume 1, ed. ESCAP Population Division. New York and Bangkok: United Nations.

Pool, Ian and Jit Cheung. 2002. "Why Were New Zealand Levels of Life-Expectation so High at the Dawn of the Twentieth Century?". Population Studies Centre, The University of Waikato. Hamilton.

Pool, Ian and Jit Cheung. 2003. "A Cohort History of Mortality." New Zealand Population Review 29(2):107-138.

Popham, Frank and Richard Mitchell. 2007. "Self-rated life expectancy and lifetime socio-economic position: cross-sectional analysis of the British household survey." International Journal of Epidemiology 36:58-65.

PPI. 2003. "Raising State Pension Age: An Update". Pensions Policy Institute. London.

PPI. 2004. "How long does a pension have to last? Briefing Note 13". Pensions Policy Institute. London.

PPI. 2006. "NPSS policy and design choices". Pensions Policy Institute. London.

PPI. 2011. "The Pensions Primer - First Tier Provision. Updated as at 12 May 2011". Pensions Policy Institute. London.

Preston, David. 2008. "Retirement Income in New Zealand: the historical context". Retirement Commission. Wellington. 
Preston, Samuel H. 1975. "The Changing Relation between Mortality and Level of Economic Development." Population Studies 29(2):231-248.

Preston, Samuel H. 1993. "The Contours of Demography." Demography 30(4):593-606.

Preston, Samuel H. 1996. "Population Studies of Mortality." Population Studies 50:525-536.

Preston, Samuel H, Patrick Heuveline and Michel Guillot. 2001. Demography: measuring and modelling population processes: Blackwell Publishing.

Preston, Samuel H. 2005. "Deadweight? - The Influence of Obesity on Longevity." The New England Journal of Medicine 352(11):1135.

Razum, Oliver and Dorothee Twardella. 2002. "Time travel with Oliver Twist Towards an explanation for a paradoxically low mortality among recent immigrants." Tropical Medicine and International Health 7(1):4-10.

Retirement Commissioner. 2007. "2007 Review of Retirement Income Policy". Commission for Financial Literacy and Retirement Income. Wellington.

Retirement Commissioner. 2010. "2010 Review of Retirement Income Policy". Commission for Financial Literacy and Retirement Income. Wellington.

Robine, J-M and M Allard. 1999. "Jeanne Calment: Validation of the Duration of Her Life." In Validation of Exceptional Longevity: Odense Monographs on Population Aging, 6, eds. Bernard Jeune and James W Vaupel: Odense University Press.

Rodway, Paul. 2010. "New Zealand superannuation scenarios for the Retirement Commission". Retirement Commission. Wellington.

Rodway, Paul and Peter Wilson. 2006. "Modelling New Zealand's Long-Term Fiscal Position". The Treasury. New Zealand Treasury Policy Perspectives Paper 06/01. Wellington.

Ross, Catherine E and John Mirowsky. 2002. "Family Relationships, Social Support and Subjective Life Expectancy." Journal of Health and Social Behavior 42 (December):469-489.

Rowland, Donald T. 2006. Demographic Methods and Concepts: Oxford University Press.

Salm, Martin. 2006. "Can Subjective Mortality Expectations and Stated Preferences Explain Varying Consumption and Saving Behaviors among the Elderly?". Bonn: Institute for the Study of Labor (IZA) Discussion Paper No. 2467. 
Sanderson, Warren C and Sergei Scherbov. 2007. "A Near Electoral Majority of Pensioners: Prospects and Policies." Population and Development Review 33(3):543554.

Schoenbaum, Michael. 1997. "Do Smokers Understand the Mortality Effects of Smoking? Evidence from the Health and Retirement Survey." American Journal of Public Health 87(5):755-759.

Scobie, Grant, John Gibson and Trinh Le. 2006. Household Wealth in New Zealand. Wellington: Institute of Policy Studies.

Shaw, Chris. 2008. "The National Population Projections Expert Advisory Group: results from a questionnaire about fertility, mortality and migration." Population Trends 134 (Winter 2008):42-53.

Shkolnikov, Vladimir, Evgeny Andreev, Zhen Zhang, James Oeppen and James Vaupel. 2011a. "Losses of Expected Lifetime in the United States and Other Developed Countries: Methods and Empirical Analyses." Demography 48(1):211-239.

Shkolnikov, Vladimir M., Dmitri A. Jdanov, Evgeny M. Andreev and James W. Vaupel. 2011b. "Steep Increase in Best-Practice Cohort Life Expectancy." Population and Development Review 37(3):419-434.

Shoven, John B and Gopi Shah Goda. 2008. "Adjusting Government Policies for Age Inflation". NBER. Working Paper 14321.

Siegel, Jacob S. 2005. "The Great Debate on the Outlook for Human Longevity: Exposition and Evaluation of Two Divergent Views". Paper for conference: Living to 100 and Beyond. Orlando, Fla.

Singh, Gopal K and Barry A Miller. 2004. "Health, Life Expectancy, and Mortality Patterns Among Immigrant Populations in the United States." Canadian Journal of Public Health 95(3).

Smith, V. Kerry. 2008. "Risk Perceptions, Optimism, and Natural Hazards." Risk Analysis 28(6):1763-1767.

Smith, V. Kerry, Donald H. Taylor Jr and Frank A. Sloan. 2001. "Longevity Expectations and Death: Can People Predict Their Own Demise?" In American Economic Review: American Economic Association.

Smits, Jeroen and Christiaan Monden. 2009. "Length of life inequality around the globe." Social Science \& Medicine 68:1114-1123.

Social Security Online. 2011. "Retirement Age Background". Social Security Administration. Washington DC. http://www.ssa.gov/pubs/background.htm. Date accessed 16 June 2011. 
Stallard, Eric. 2006. "Demographic Issues in Longevity Risk Analysis." The Journal of Risk and Insurance 73(4):575-609.

Statistics Canada. 2010. "Population Projections for Canada, Provinces and Territories". Statistics Canada. Ottawa.

Statistics New Zealand. 2007. "New Zealand's life expectancies on a world stage: a cohort perspective." In Demographic Trends 2006. Wellington: Statistics New Zealand.

Statistics New Zealand. 2008. "How accurate are population projections? An evaluation of Statistics New Zealand population projections, 1991-2006". Statistics New Zealand. Wellington.

Statistics New Zealand. 2009a. "Information about the demographic projections". http://www2.stats.govt.nz/domino/external/omni/omni.nsf/outputs/Demographic+Pr ojections\#PA. Date accessed 18 January 2010.

Statistics New Zealand. 2009b. "Labour force participation of New Zealanders aged 65 years and over, 1986-2006". Statistics New Zealand. Wellington.

Statistics New Zealand. 2009c. "New Zealand Life Tables: 2005-7". Statistics New Zealand. Wellington.

Stewart, S, D Cutler and A Rosen. 2009. "Forecasting the Effects of Obesity and Smoking on U.S. Life Expectancy." The New England Journal of Medicine 361(23):2252-2260.

Stoto, Michael A. 1988. "Dealing with Uncertainty: Statistics for an Aging Population." The American Statistician 42(2):103-110.

Su, Dejun. 2009. "Risk Exposure in Early Life and Mortality at Older Ages: Evidence from Union Army Veterans." Population and Development Review 35(2):275-295.

The Actuarial Profession. 2006. "The response of the actuarial profession to the Second Report of the Pensions Commission A New Settlement for the Twenty-First Century. Paper 1: Commentary on the use of life expectancy data in the Pensions Commission Report". London.

The Lancet. 2011. "An epidemic of risk factors for cardiovascular disease." The Lancet 377(9765):527-527.

The Treasury. 2006. "New Zealand's Long-Term Fiscal Position". The Treasury. Wellington. 
The Treasury. 2009. "Challenges and Choices: New Zealand's Long-term Fiscal Statement". The Treasury. Wellington.

Tobias, Martin, Tony Blakely, Don Matheson, Kumanan Rasanathan and June Atkinson. 2009. "Changing trends in indigenous inequalities in mortality: lessons from New Zealand." International Journal of Epidemiology 38(6):1711-1722.

Tokor, Alexander and Vincent M Murphy. 1967. "Some psychological correlates of subjective life expectancy." Journal of Clinical Psychology 23:21-24.

Tuljapurkar, Shripad. 2005. "Future Mortality: A Bumpy Road to Shangri-La?" Science of Aging Knowledge Environment 2005(14):pe9.

Tuljapurkar, Shripad and Ryan D Edwards. 2011. "Variance in death and its implications for modeling and forecasting mortality." Demographic Research 24(21):497-526.

Tuljapurkar, Shripad, Nan Li and Carl Boe. 2000. "A universal pattern of mortality decline in the G7 countries." Nature 405(15 June 2000):789-792.

TVNZ. 2010. "Retirement age rise mooted". 7 December 2010. http://tvnz.co.nz/national-news/retirement-age-rise-mooted-3951957. Date accessed 16 June 2011.

UK Government. 2011. "Calculating your State Pension age". Directgov. http://www.direct.gov.uk/en/Pensionsandretirementplanning/StatePension/DG_4017 919. Date accessed 7 November 2011.

United Nations. 2007. "World Population Ageing 2007". Population Division United Nations Department of Economic and Social Affairs. United Nations. New York.

United Nations. 2011. "World Population Prospects: The 2010 Revision CD-ROM Edition." Population Division of the Department of Economic and Social Affairs of the United Nations Secretariat.

US Census Bureau. 2008. "2008 National Population Projections". US Census Bureau.

van den Berg, Gerard, Gabriele Doblhammer-Reiter and Kaare Christensen. 2011. "Being Born Under Adverse Economic Conditions Leads to a Higher Cardiovascular Mortality Rate Later in Life: Evidence Based on Individuals Born at Different Stages of the Business Cycle." Demography 48(2):507-530.

van Solinge, Hanna and Kene Henkens. 2009. "Living longer, working longer? The impact of subjective life expectancy on retirement intentions and behaviour." European Journal of Public Health Advance Access published October 12, 2009. 
Vaupel, J. W. 1986. "How Change in Age-Specific Mortality Affects Life Expectancy." Population Studies 40(1):147-157.

Vaupel, James. 2001. "Demographic Insights into Longevity." Population: An English Selection 13(1):245-260.

Vaupel, James W. 2009. "Lively Questions for Demographers about Death at Older Ages." Population and Development Review 35(2):347-356.

Vaupel, James W, James R Carey, Kaare Christensen, Thomas E Johnson, Anatoli I Yashin, Niels V Holm, Ivan A Iachine, Vaino Kannisto, Aziz A Khazaeli, Pablo Liedo, Valter D Longo, Yi Zeng, Kenneth G Manton and James W Curtsinger. 1998.

"Biodemographic Trajectories of Longevity." Science 280(8 May 1998):855-860.

Viner, Russell M, Carolyn Coffey, Colin Mathers, Paul Bloem, Anthony Costello, John Santelli and George C Patton. 2011. "50-year mortality trends in children and young people: a study of 50 low-income, middle-income, and high-income countries." The Lancet 377 (April 2, 2011):1162-1130.

Wack, Pierre. 1985a. "Scenarios: shooting the rapids." Harvard Business Review 63(6):139-150

Wack, Pierre. 1985b. "Scenarios: unchartered waters ahead." Harvard Business Review 63(5):73-89

Wang, Y. Claire, Klim McPherson, Tim Marsh, Steven L. Gortmaker and Martin Brown. 2011. "Health and economic burden of the projected obesity trends in the USA and the UK." The Lancet 378(9793):815-825.

Wardle, J and A Steptoe. 2003. "Socioeconomic differences in attitudes and beliefs about health lifestyles." Journal of Epidemiology and Community Health 57:440-443.

White, Kevin M. 2002. "Longevity Advances in High-Income Countries, 1955-96." Population and Development Review 28(1):59-76.

WHO. 2008. "Closing the gap in a generation: Health equity through action on the social determinants of health. Final Report of the Commission on the Social Determinants of Health". World Health Organisation. Geneva.

Wild, S. H., C. Fischbacher, A. Brock, C. Griffiths and R. Bhopal. 2007. "Mortality from all causes and circulatory disease by country of birth in England and Wales 20012003." J Public Health 29(2):191-198.

Willcox, Bradley J, D Craig Willcox, Qimei He, J David Curb and Makoto Suzuki. 2006. "Siblings of Okinawan Centenarians Share Lifelong Mortality Advantages." The Journals of Gerontology 61A(4). 
Willets, R C. 2004. "The Cohort Effect: Insights and Explanations". Institute of Actuaries and Faculty of Actuaries. London.

Willets, R C, Adrian Gallop, P A Leandro, J L C Lu, A S MacDonald, K A Miller, S J Richards, N Robjohns, J P Ryan and H R Waters. 2004. "Longevity in the 21st century". Presented to the Faculty of Actuaries 15 March 2004 and to the Institute of Actuaries 26 April 2004. The Actuarial Profession. London.

Wilmoth, J. R. 2000. "Demography of longevity: past, present, and future trends." Experimental Gerontology 35(9-10):1111-1129.

Wilmoth, John R. 1998. "The Future of Human Longevity: A Demographer's Perspective." Science 280(5362):395-397.

Wilmoth, John R. 2005. "On the relationship between period and cohort mortality." Demographic Research 13(11):231-280.

Wilson, Chris. 2011. "Understanding Global Demographic Convergence since 1950." Population and Development Review 37(2):375-388.

World Bank. 1994. "Averting the old age crisis: policies to protect the old and promote growth". The International Bank for Reconstruction and Development, The World Bank, Oxford University Press. Washington DC.

Young, Christabel M. 1991. "Changes in the Demographic Behaviour of Migrants in Australia and the Transition between Generations." Population Studies 45(1):67-89.

Young, Ken, Deborah Ashby, Annette Boaz and Lesley Grayson. 2002. "Social Science and the Evidence-based Policy Movement." Social Policy and Society 1(3):215-224.

Zhivan, Natalia, Steven A Sass, Margarita Sapozhnikov and Kelly Haverstick. 2008.

"An "Elastic" Earliest Eligibility Age for Social Security". Center for Retirement Research at Boston College. February 2008, Number 8-2. 